\title{
WestVirginiaUniversity
}

THE RESEARCH REPOSITORY @ WVU

Graduate Theses, Dissertations, and Problem Reports

2000

\section{Composition effects on macroscopic solidification segregation of superalloys}

Wei Chen

West Virginia University

Follow this and additional works at: https://researchrepository.wvu.edu/etd

\section{Recommended Citation}

Chen, Wei, "Composition effects on macroscopic solidification segregation of superalloys" (2000).

Graduate Theses, Dissertations, and Problem Reports. 1229.

https://researchrepository.wvu.edu/etd/1229

This Dissertation is protected by copyright and/or related rights. It has been brought to you by the The Research Repository @ WVU with permission from the rights-holder(s). You are free to use this Dissertation in any way that is permitted by the copyright and related rights legislation that applies to your use. For other uses you must obtain permission from the rights-holder(s) directly, unless additional rights are indicated by a Creative Commons license in the record and/ or on the work itself. This Dissertation has been accepted for inclusion in WVU Graduate Theses, Dissertations, and Problem Reports collection by an authorized administrator of The Research Repository @ WVU.

For more information, please contact researchrepository@mail.wvu.edu. 


\title{
Composition Effects on Macroscopic Solidification Segregation of Superalloys
}

\author{
Wei Chen
}

Dissertation submitted to the College of Engineering and Mineral Resources

at West Virginia University in partial fulfillment of the requirements

for the degree of

\author{
Doctor of Philosophy \\ in \\ Mechanical Engineering \\ Keh-Minn Chang, Ph.D., Chair \\ Ed Sneckenberger, Ph.D. \\ Eric Johnson, Ph.D. \\ Victor Mucino, Ph.D. \\ John deBarbadillo, Ph.D. \\ Department of Mechanical and Aerospace Engineering
}

Morgantown, West Virginia

2000 


\section{ABSTRACT \\ Composition Effects on Macroscopic Solidification Segregation of Superalloys}

\section{Wei Chen}

This research work investigates primarily the composition effects on solidification macrosegregation, i.e. freckles, in superalloys. First, the freckling mechanism of superalloys is developed using the physical simulation of freckle formation in specially designed model alloys. It is found that freckles originate from dendrite irregularities at the solidification front. The freckle channels flow downward in $\mathrm{Nb}$-containing alloys and upward in T-containing alloy under horizontal solidification conditions. The horizontal solidification correctly simulates the horizontal component in remelt ingot where freckling potential is the highest. Based on the result, key control parameters for freckling are identified.

Thermodynamic simulation approach is employed to study the solidification behavior of superalloys. The theoretical prediction yields relative good agreement with experimental observations of freezing range, solidification sequence, and the occurrence of secondary phases. Solidification diagram of Ni-Cr-Fe-Nb alloy system is developed using the simulation approach to predict the phase relationship during solidification. The simulation results can be used as inputs for freckling criteria.

The sensitivity of freckling criteria relies on the accurate acquisition of the attributes that are critical to the key control parameters. These attributes include interdendritic liquid composition, temperature and fraction of solid. Two techniques are developed in the present study to obtain their correlation. From here, liquid composition and fraction solid at any temperature in the mushy zone can be obtained.

Composition effects on the key control parameters are investigated in detail. Liquid density during solidification depends on the composition and the temperature of the liquid. A significant temperature effect on density change is found. Regression analysis shows that $\mathrm{Nb}$ has more pronounced effect than $\mathrm{Ti}$ in reducing the fraction solid. The dendrite arm spacing and the solidification front angle are largely dependent on the processing conditions.

Freckling criterion in remelting of superalloys is developed at the end of this study. A composition related is proposed to account for the alloying effect on freckling tendency. The model correctly predicts the freckling pattern in Ti- and $\mathrm{Nb}$-containing model alloys. It also correctly predicts the freckling tendency of industrial alloys. 


\section{Acknowledgment}

I am sincerely grateful to my advisor, Dr. Keh-Minn Chang, for his knowledgeable guidance and encouragement for this research project. I would like to thank Dr. Wanhong Yang for his continuous help and inspiring discussions during the course of this work. I would also like to thank Dr. Jack deBarbadillo for his devoted support for this project.

Special thanks are due to Dr. Sarwan Mannan for his continuous assistance and advice of the project, and to Walt Schrodder, Rick Corn, and their team at Technology Processing Center (TPC), Special Metals Corporation, Huntington, WV, for their help in preparing the model alloys.

I sincerely appreciate Koki Morita and Daido Steel Corporation, Japan, for their help to run the horizontal solidification.

Thanks are also due to Joel Harris and Diane Schwegler-Berry, NIOSH, Morgantown, WV, for their time, patience and technical support with SEM/EDS. 
To my wife, Chen Cong. 


\section{Table of Contents}

\section{Chapter 1: Introduction}

1.1. Background 2

1.2. Freckles in Superalloys 2

1.2.1. Structure of Freckles 2

1.2.2. Detrimental Effects 3

1.3. Mechanism of Freckle Formation 4

1.3.1. Density Inversion Theory 4

1.3.2. Freckling in Directional Solidification 5

$\begin{array}{ll}\text { 1.3.3. Freckling in Remelting } & 6\end{array}$

1.3.4. Controlling Factors $\quad 7$

1.4. Freckling Criteria $\quad 8$

1.4.1. Local Solidification Time $\quad 8$

1.4.2. Flemings' Criterion 9

1.4.3. Rayleigh Number 9

1.5. Composition Effects 11

1.5.1. Solute Redistribution in Dendritic Solidification 11

1.5.2. Analog Systems 12

1.5.3. Ni-based Superalloys 13

1.6. Objectives of Research 14

\section{Chapter 2: Experimental}

2.1. Introduction 20

2.2. Alloy Selection 20

2.2.1. Model Alloys 20

2.2.2. Industrial Alloy 21

2.3. Horizontal Solidification 21

2.4. Differential Thermal Analysis 22

2.5. Metallography 23

2.5.1. Sample Preparation 23

2.5.2. Optical Microscope 23

2.5.3. Scanning Electron Microscope 23

2.5.4. Energy Dispersive Spectrometry 24

2.6. CALPHAD 24

2.6.1. Thermo-Calc ${ }^{\mathrm{TM}} 24$

2.6.2. NiFe-Database 25

2.7. Liquid Density Calculation 26

2.8. Summary 27

Chapter 3: Freckling Mechanism in Ni-based Superalloys

3.1. Introduction 32

3.2. Freckling in an Alloy 625 Ingot 32

3.2.1. Freckle Appearance 33 
3.2.2. Discussion $\quad 34$

3.3. Physical Simulation of Freckling in Model Superalloys 34

3.3.1 Freckle Appearance $\quad 35$

$\begin{array}{ll}3.3 .2 & \text { Channel Initiation } \\ 3.3 .3 & 37\end{array}$

$\begin{array}{lll}3.3 .3 & \text { Temperature Data } & 38\end{array}$

3.3.4 Secondary Dendrite Arm Spacing $\quad 39$

3.4. Mechanism of Freckle Formation 40

3.4.1. Isotherms 41

3.4.2. Solidification Front (Freezing Front) 42

3.4.3. Iso-fraction Solid 43

3.4.4. Freckling Mechanism 43

3.4.5. Application to Remelting Process 45

3.5. Key Controlling Parameters 46

$\begin{array}{ll}\text { 3.6. Summary } & 47\end{array}$

Chapter 4: Solidification in Ni-based Model Superalloys: CALPHAD Approach

4.1. Introduction 66

4.2. Approaches 66

4.2.1. Model Superalloys 66

4.2.2. CALPHAD method in Solidification Simulation 67

4.3. Solidification Diagram of Ni-Cr-Fe-Nb System 69

4.3.1. Ternary Systems $\quad 69$

4.3.2. Quaternary System: CALPHAD Approach 70

4.3.3. Experimental Results $\quad 70$

\begin{tabular}{ll} 
4.3.4. & Alloying Effects \\
\hline 2
\end{tabular}

4.4. Freezing Range of Model Alloys $\quad 73$

4.4.1. Freezing Range 74

4.4.2. Alloying Effects $\quad 75$

4.5. Solidification Sequence and Secondary Phases 76

4.5.1. Solidification Sequence 76

$\begin{array}{ll}\text { 4.5.2. Secondary Phases } & 77\end{array}$

$\begin{array}{ll}\text { 4.5.3. Alloying Effects } & 78\end{array}$

$\begin{array}{lll}\text { 4.6. Summary } & \text { Su }\end{array}$

Chapter 5: Composition, Temperature and Fraction Solid in Solidification: Experimental Determination

5.1. Introduction 90

5.2. Solute Redistribution 91

5.2.1. Microsegregation Profiles $\quad 92$

5.2.2. Partition Ratio $\quad 93$

5.3. Fraction of Solid 94

5.3.1. Quantitative DTA 96

5.3.2. Model Alloys $\quad 97$

$\begin{array}{ll}\text { 5.3.3. Alloying Effects } & 97\end{array}$

5.4. Secondary Dendrite Arm Spacing 98 
5.5. Discussion 99

5.5.1. Kinetic Effects 99

5.5.2. Experiments versus CALPHAD 101

$\begin{array}{ll}\text { 5.6. Summary } & 102\end{array}$

\section{Chapter 6: Freckling Criterion}

6.1. Introduction $\quad 115$

6.2. Rayleigh Criteria $\quad 115$

6.3. Sensitivity of Key Control Parameters 118

6.3.1. Viscosity 118

6.3.2. Permeability 118

$\begin{array}{ll}\text { 6.3.3. Liquid Density } & 119\end{array}$

6.3.4. Fraction of Solid 120

6.4. Composition Effects $\quad 121$

6.4.1. Driving Force 121

6.4.2. Resistance 122

6.5. Freckling Tendencies in Model Alloys $\quad 124$

$\begin{array}{ll}\text { 6.5.1. Relative Rayleigh Number } & 124\end{array}$

6.5.2. Freckling Tendencies in Model Alloys 124

6.6. Freckling in Industrial Alloys 126

6.7. Discussion 126

6.7.1. Processing Conditions 126

$\begin{array}{ll}\text { 6.7.2. Ingot Size } & 127\end{array}$

$\begin{array}{lll}\text { 6.8. Summary } & 127\end{array}$

Chapter 7: Conclusions 136

$\begin{array}{ll}\text { Appendix A } & 138\end{array}$

Appendix B 141

References $\quad 143$ 


\section{List of Tables}

Table 1-1 Chemical compositions of alloys reported for freckling (wt pct)

Table 2-1 Chemical compositions of model superalloys (wt pct, $\mathrm{Ti} / \mathrm{Nb}$ in at 28 pct)

Table 2-2 Chemical compositions of industrial alloys 718, 706 and 625 (wt 28 pct)

Table 3-1 Nominal and Actual Chemical Compositions of Alloy 625 (wt.\%) 32

Table 3-2 Chemical Composition of Phases in Freckles in Alloy 625 (at pct) 34

Table 3-3 SDAS of horizontal solidification ingots $(\mu \mathrm{m}) \quad 40$

Table 4-1 Calculated Solidification Sequences and Amounts of Eutectic 70

Table 4-2 Average Eutectic Composition in Experimental Alloys by EDS (wt 71 pct)

Table 4-3 Freezing range (TL-TS) obtained using Thermo-Calc and DTA 80 ( C)

Table 4-4 Correlation of difference in characteristic temperature (Between 75 DTA and Thermo-Calc) and alloy compositions.

Table 4-5 Results of Thermo-Calc simulation of Ni-Cr-Fe-Nb-Ti-Al system $\quad 88$

Table 5-1 Average solid composition using systematic point count method. 104

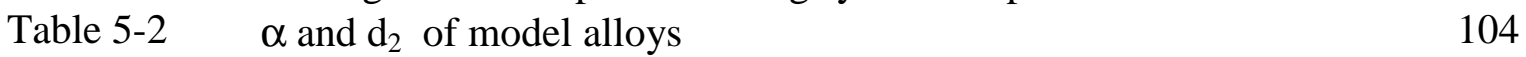

Table 5-3 Correlation of CALPHAD and experimental results. 101

Table 6-1 The breakdown of liquid density changes in model alloys 122

Table 6-2 Melt Rate Excursion of VAR 718 133 


\section{List of Figures}

Figure 1-1 Freckles in DS MM200.

Page \#

Figure 1-2 Freckles in VAR alloy 706 ingot. 16

Figure 1-3 SEM microstructure of freckles in ESR alloy 718. 16

Figure 1-4 SEM of extracted phases from freckled area. (a) NbC carbide; (b) 16 Laves; (c) $\eta$.

Figure 1-5 (c) Composition and (b) density profiles through (b) an upward growing dendrite. (a) Trace $\mathrm{A}$ along a dendrite axis and trace $\mathrm{B}$, interdendritic, with reference to a phase diagram (a).

Figure 1-6 (top) A phase diagram with limited solid solubility, C*; (bottom) the dependence of freckle potential on composition.

Figure 1-7 Schematic showing the density inversion theory and interface 17 perturbation.

Figure 1-8 Interdendritic flow pattern in remelting by Mehrabian [13]. 18

Figure 1-9 Composition effects on freckling in $\mathrm{Pb}-\mathrm{Sb}$ and $\mathrm{Pb}-\mathrm{Sn}$ binary 18 systems.

Figure 2-1 Isopleth of (a) Ni-20Cr-Ti, (b) Ni-20Cr-Nb, and (c) Ni-20Cr-18Fe- 29 $\mathrm{Nb}$, calculated by Thermo-Calc.

Figure 2-2 Schematic of horizontal solidification setup (top view and side view).

Figure 3-1 Cut-up surfaces showing freckles decorating pool profiles. 47

Figure 3-2 Macrostructure of transverse (left) and longitudinal (right) sections 47 of freckles.

Figure 3-3 Scanning electron microscopy of freckled area. 48

Figure 3-4 Segregation and phases in freckled area of alloy 625. 48

Figure 3-5 Quantitative EDS analysis on the line-scan across the freckled area. 49

Figure 3-6(a) Longitudinal cross section of HV8851 revealing freckles. 50

Figure 3-6(b) Longitudinal cross section of HV8852 revealing freckles. 51

Figure 3-6(c) Longitudinal cross section of HV8853 revealing freckles. 52

Figure 3-7 Cut-up plans for (a) HV8851, (b) HV8852, and (c) HV8853. 53

Photos show the longitudinal cross-section and $\mathrm{d}_{2}$ were measured on the transverse cross sections.

Figure 3-8 Freckles in HV8851. (a) Optical microphotograph (double arrow indicates the growth direction); (b) shows the secondary electron images of one freckle in (a); (c) is the enlarged area of the lower region in (b); (d) is the back-scattered electron image of the enlarged area of the upper region in (b).

Figure 3-9 Optical photomicrographs of (a) transverse and (b) longitudinal cross sections of freckles in HV8852. (Double arrow is the growth direction and single arrow is gravity direction). (c) is the SEM image of longitudinal section of freckles, solidus side is on the left. (d) is the back-scattered electron image of eutectic constituents in freckled area. 
Figure 3-10 Optical photomicrographs of (a) transverse and (b) longitudinal cross sections of freckles in HV8853. (Double arrow is the growth direction and single arrow is gravity direction). (c) shows the SEM image of transverse cross section of freckle (growth direction points upward.) (d) shows the eutectic constituents in freckled area.

Figure 3-11 Freckle initiation in HV8851. Optical photomicrograph shows two freckle trials originating from bottom of the ingot.

Figure 3-12 Longitudinal section of freckles in HV8853 showing the irregularities of dendrites and downward freckle flow. (Gravity force points downwards and ingot grows to the left)

Figure 3-13 Temperature profiles of (a) HV8851 (Liq. $=1373^{\circ} \mathrm{C}$ ), (b) $\mathrm{HV} 8852$ (Liq. $=1364^{\circ} \mathrm{C}$ ), and (c) $\mathrm{HV} 8853\left(\mathrm{Liq} .=1351^{\circ} \mathrm{C}\right)$. Dash lines are the liquidus temperatures of the model alloys.

Figure 3-14 Positions of liquidus and isotherms that are $50^{\circ} \mathrm{C}\left(20^{\circ} \mathrm{C}\right)$ below liquidus of (a) HV8851, (b) HV8852, and (c) HV8853.

Figure 3-15 (a) Cooling rate, (b) thermal gradient, and (c) moving speeds of model alloys during horizontal solidification.

Figure 3-16 Secondary dendrite arm spacings versus distance from chill in horizontally solidified model alloys (HV8852: Ni-20Cr-8.6Nb and HV8853: Ni-20Cr-18Fe-7Nb).

Figure 3-17 Horizontal solidification of binary $\mathrm{Al}-20 \mathrm{Cu}$ at different intervals during solidification. Chill is on the right hand side and crystal grows from right to left [44].

Figure 3-18 Schematics of isotherms, solidification front, iso-fraction solid and freckle flow in the mushy zone of horizontal solidification of (a) Ti-containing and (b) Nb-containing model alloys. In Ticontaining alloy, iso-fraction solids are parallel to isotherms.

("C" $\mathrm{C}$ stands for chill surface, "s.f." is the solidification front.)

Figure 3-19 Schematic phase diagram shows that for two liquid alloys and the same temperature drop from liquidus, the fraction of solid of alloy 1 is greater than that of alloy 2 . Here the fraction of solid is defined as the ratio of bc/ac for alloy 1 , and ef/df for alloy 2 . Since bc/ac > ef/df, fraction solid of alloy 1 is greater than alloy 2 .

Figure 4-1 Schematic of Scheil solidification module in Thermo-Calc.

Figure 4-2 Liquidus projection diagrams of (a) $\mathrm{Ni}-\mathrm{Cr}-\mathrm{Nb}$ and (b) $\mathrm{Ni}-\mathrm{Fe}-\mathrm{Nb}$ systems calculated using Thermo-Calc. The projection of monovariant eutectic lines onto the $\mathrm{Nb}$ vs. temperature plane of both systems is given in (c).

Figure 4-3 Scheil solidification simulation of HV8853, (a): fraction solid vs. temperature and (b): liquid composition vs. fraction solid.

Figure 4-4 Pseudo-ternary solidification diagram of Ni-Cr-Fe-Nb alloy system. 82

Figure 4-5 DTA cooling curve of HV8853 at 20 and $5 \mathrm{C} / \mathrm{min}$ showing 83 eutectic reactions.

Figure 4-6 Back-scattered electron images of HV8853 (DTA, 5 C/min). 83

Figure 4-7 EDS spectrums of eutectic phases in alloy CL-3. 83 
Figure 4-8 Back-scattered electron images of as-cast CK-30.

Figure 4-9 (a) and (b) are BSE images of CL-2 where the bright phases are $\mathrm{Ni}_{3} \mathrm{Nb}$. (c) and (d) are BSE images of CL-3 where the bright phases are $\mathrm{Ni}_{3} \mathrm{Nb}$.

Figure 4-10 Solidification diagram of Ni-Cr-Fe-Nb showing alloying effects on formation of secondary phases. $\mathrm{AB}:[\mathrm{Cr}]+[\mathrm{Fe}]+0.9[\mathrm{Nb}]=44$

Figure 4-11 Correlation of freezing range data determined by DTA and Thermo-Calc.

Figure 4-12 Regression results of freezing range data of Thermo-Calc and DTA.

Figure 4-13 (a) and (b): Back-scattered electron images of alloy HV8582, (c): DTA cooling curve of HV8582 showing two eutectic reactions.

Figure 4-14 706 -type of model alloys all exhibit Laves phase as the only eutectic constituent. (a) HV8523, (b) HV8524, and (c) HV8552.

Figure 4-15 (a) Back-scattered electron image and (b) DTA cooling curve of HV8551.

Figure 5-1 Dendrite structure in Ni-based superalloys (Back-scattered electron image).

Figure 5-2 Schematic of monototnic increase of solid $\mathrm{Nb}$ concentration in model alloys.

Figure 5-3 Solid composition profiles in model alloys (a) HV8851, (b) 105 HV8852, and (c) HV8853.

Figure 5-4 Liquid composition profiles in model alloys (a) HV8851, (b) HV8852, and (c) HV8853. HV8852, and (c) HV8853.

Figure 5-6 Characteristic features of fs(T) curve in superalloys. 108

Figure 5-7 Principle of quantitative DTA. 108

Figure 5-8 fs(T) curves of HV8851, HV8852 and HV8853 by Thermo-Calc (left) and quantitative DTA analysis (right).

Figure 5-9 fs(T) curves of HV8520 and HV8549 by Thermo-Calc (left) and quantitative DTA analysis (right).

Figure 5-10 fs(T) curves of HV8551, HV8550, HV8520 and HV8521 by Thermo-Calc (left) and quantitative DTA analysis (right).

Figure 5-11 fs(T) curves of HV8523, HV8524, HV8552 and HV8586 by Thermo-Calc (left) and quantitative DTA analysis (right).

Figure 5-12 Comparison of quantitative DTA with quenching results in alloys 718 and 625.

Figure 5-13 Fitting $\mathrm{f}_{\mathrm{L}}(\Delta \mathrm{T})$ curve using exponential function.

109

110

110

Figure 5-14 Correlation of $\alpha$ with $\mathrm{Ti}$ (left) and $\mathrm{Nb}$ (right) concentrations. Circled data points (ternary alloys HV8851 and HV8852) are outliers and excluded from regression.

Figure 5-15 Comparison of predicted and actual $\alpha$ values in superalloys. 112

Figure 5-16 Secondary dendrite arm spacings versus (a) Ti/Nb atomic ratio, (b) 113 $\mathrm{Ti}$ content, and (c) $\mathrm{Nb}$ content in model alloys.

Figure 6-1 Schematic illustration of volume element during remelt 129 solidification and mass balance in the volume element inside 
mushy zone.

Figure 6-2 Schematic illustration of change in flow behavior in the mushy zone during solidification under vertical DS (with density inversion) and remelt (without density inversion) conditions.

Figure 6-3 Flowdown of controlling factors of freckling in remelt processing of superalloys.

Figure 6-4 Liquid density change versus temperature difference of model 130 alloys.

Figure 6-5 Permeability vs. temperature difference of model alloys (no $\mathrm{d}_{2}$ considered).

Figure 6-6 Relative $\mathrm{Ra}^{*}$ versus temperature difference of model alloys (no $d_{2} \quad 131$ considered).

Figure 6-7 Ra* versus fraction of solid of model alloys (no $d_{2}$ considered).

Figure 6-8 Maximum Ra versus distance from chill of horizontally solidified model alloys HV8852 and HV8853 $\left(d_{2}\right.$ and $\theta$ are considered in calculation).

Figure 6-9 Liquid density change versus temperature difference of industrial alloys.

Figure 6-10 Permeability vs. temperature difference of industrial alloys (no $d_{2}$ considered).

Figure 6-11 Relative Ra* vs. temperature difference of industrial alloys (no $d_{2} \quad 134$ considered).

Figure 6-12 Ra* versus fraction of solid of model alloys (no $d_{2}$ considered).

Figure B-1 (a) $\mathrm{Nb}$ composition versus scattered data and (b) $\mathrm{Nb}$ composition versus fraction solid profile obtained in alloy 718 DTA sample. 


\section{Chapter 1}

\section{Introduction}

1.1. Background 2

1.2. Freckles in Superalloys 2

1.2.1. Structure of Freckles 2

1.2.2. Detrimental Effects 3

1.3. Mechanism of Freckle Formation 4

1.3.1. Density Inversion Theory 4

1.3.2. Freckling in Directional Solidification 5

1.3.3. Freckling in Remelting 6

1.3.4. Controlling Factors 7

1.4. Freckling Criteria $\quad 8$

1.4.1. Local Solidification Time $\quad 8$

1.4.2. Flemings' Criterion 9

1.4.3. Rayleigh Number 9

1.5. Composition Effects 11

1.5.1. Solute Redistribution in Dendritic Solidification $\quad 11$

1.5.2. Analog Systems 12

1.5.3. Ni-based Superalloys $\quad 13$

1.6. Objectives of Research 14 


\subsection{Background}

Large, more aggressive jet engines and industrial gas turbine designs have recently increased the demand for large diameter superalloy ingots for rotating component applications [1,2]. Commercial ingot production utilizes sequence of one or more secondary melting (remelting) processes, including electroslag remelting (ESR) and vacuum arc remelting (VAR). These processes are used to produce quality ingots following primary melt step, but they can also be a source of defects due to the complicated operation and the highly complex composition of the alloys. The ingot diameter has been limited by the strong tendency these alloys have towards the formation of melt-related defects $[2,3]$.

Typical ingot defects in Ni-based superalloys can be characterized as either inclusions or segregation-related defects. Examples of segregation defects include freckles (areas that are enriched in hardening elements) and white spots (areas that are lean in hardening elements) $[2,4]$. Inclusions are primarily clusters of oxides and nitrides.

Over the past three decades, there have been substantial efforts by both industry and academic institutes to reduce the segregation defects and control of freckles is always the critical issue [1-9]. Experimental results on freckle formation and freckling mechanisms in Ni-based superalloys and analog systems are reviewed through extensive literature survey and inputs from industry. Descriptions and limitations of various freckling criteria reported in literature are discussed. Based on the summary, the objectives of research are proposed at the end of this chapter.

\subsection{Freckles in Superalloys}

\subsubsection{Structure of Freckles}

Freckles have been observed in two types of commercial casting practices, i.e. directional solidification and large ingot remelting. Freckles appear as long vertical chains of equiaxed grains significantly enriched in normally segregating elements in directionally solidified (DS) polycrystalline or single crystal castings [5,6]. Freckle lines are usually located on the exterior surface of the casting and associated with micro-porosity and 
eutectic phase particles (Figure 1-1). Cross sections of freckle lines reveal that they are as deep as they are wide $(1 \sim 2 \mathrm{~mm})$. Freckle lines are evenly distributed around the circumference of a cylindrically shaped casting. For a turbine blade, they prefer to occur at root section (thicker section) rather than airfoil (thinner section), which indicates a size effect of the casting [7].

In ESR and VAR ingots, freckle lines are nearly aligned with the pool profile and are located in the center to mid-radius region, as shown in Figure 1-2 [2]. They appear as dark spots when etched on the cross sections of ingots and billets due to the existence of large amount of eutectic constituents in Nb-containing alloys. A close-up of freckles in alloy 718 (Figure 1-3) shows that it contains excessive amount of Laves phase that forms a network outlining the interdendritic areas [8]. Extracted phase particles from the freckles in alloy 706 are illustrated in Figure 1-4 [9]. Freckles can be found in both ESR and VAR, but with a higher incidence in ESR. This has been attributed to different liquid pool shapes, i.e. "U"-shaped pool profile in VAR and "V"-shaped pool in ESR, and higher melt rate in ESR [8].

Two fundamental differences exist between DS and remelting processed superalloys. First, significantly larger remelt ingots (such as VAR alloy 718 of $508 \mathrm{~mm}$ in diameter) are continuously melted and solidified. A convex shaped liquid pool rather than a planar growth front remains at the solidification front throughout the solidification process in VAR or ESR. Shop experience indicates that freckles are most likely to occur in the region of the ingot where the solidification direction is more or less horizontal. The term "remelting" specifically refers to the continuous solidification process where the sloped solidification front exists. Second, many of the remelting processed superalloys contain $\mathrm{Nb}$ and Mo as major solute elements, while DS alloys have high contents of $\mathrm{Al}, \mathrm{Ti}$, and refractory elements ( $\mathrm{Ta}, \mathrm{W}$, etc.). These solute elements are either positive (segregate preferentially into the liquid, such as $\mathrm{Nb}$ and $\mathrm{Mo}$ ), or negative (segregate preferentially into the solid, such as W) segregating elements. There are other differences that may not be clearly defined, for example, the transients during the remelting process. The differences contribute to different freckling mechanisms though freckle chemistries are shifted towards eutectic compositions in both cases. 


\subsubsection{Detrimental Effects}

Freckles in full size ingot have a size at least 3 or 4 order of magnitude larger than that of microsegregation. After forging and heat treatment processes, these inhomogeneous and discontinuous defects remain in the final product and usually lie along the forging flow lines. The orientation of the freckle channels has a significant effect on the properties. Mechanical properties such as tensile, fracture toughness, and fatigue crack growth are significantly degraded when longitudinal direction of freckle channels is perpendicular to the loading axis. Excessive amount of incoherent particles (such as the faceted carbidenitrides) and chain-like Laves networks in freckles may contribute to the degradation due to their crack-initiation features. In addition some freckles can develop cracks during the forging process leading to pre-existing flaws in the part [9].

\subsection{Mechanism of Freckle Formation}

\subsubsection{Density Inversion Theory}

Flemings and co-workers first demonstrated the importance of fluid flow in macrosegregation in a series of papers [10-13], aimed initially at description of inverse segregation. McDonald and Hunt [14,15], Copley et al [6] used the $\mathrm{NH}_{4} \mathrm{Cl}-\mathrm{H}_{2} \mathrm{O}$ analogue system to investigate freckle formation and proposed that freckle segregates were the result of interdendritic fluid flow driven by density inversion during directional solidification. The mechanism has, at various times, been applied to non-metallic analogues and low melting-point alloys such as the $\mathrm{Pb}-\mathrm{Sn}$ and $\mathrm{Al}-\mathrm{Cu}$ systems for the explanation of freckle formation, so as named "density inversion theory" [16-20].

During the solidification of an alloy, a dendritic array forms in the mushy zone between the liquid (fraction of solid, $f_{S}=0$ ) and the solid $\left(f_{S}=1\right)$. Both temperature and concentration gradients exist across the mushy region from the top to the bottom, as illustrated in Figure 1-5 (a) and (b). The density of the interdendritic liquid, which depends on both the thermal and solutal effects, can be described as following [21]:

$$
\rho=\rho_{0} \times\left[1+\alpha\left(T-T_{0}\right)+\beta\left(C-C_{0}\right)\right]
$$

Where $\rho_{0}$ is the liquid density of the alloy $C_{0}$ at liquidus temperature $T_{0}, \alpha$ is the thermal volume expansion coefficient, and $\beta$ is the solutal volume expansion coefficient. In most 
cases, $\alpha$ is positive (i.e., liquid density increases when temperature decreases), while $\beta$ can be either positive or negative, depending on the solute element. When the negative effect of $\beta$ on density outweighs the temperature effect, the liquid density decreases with the progress of solidification, as shown in Figure 1-5 (d). The inverse density distribution becomes thermodynamically unstable when the gravity force is acting against the growth direction of solid. As a result, fluid flow may occur due to the density inversion. From the discussion a freckling potential can be given by [6],

$$
\begin{aligned}
& \Delta \Psi=\frac{g \beta}{6 \alpha} \frac{\left(T-T_{S}\right)\left(C_{S}-C\right)\left(\rho-\rho_{S}\right)}{G^{2}} \propto C_{L}^{3}, \quad 0 \leq C \leq C^{*} \\
& \Delta \Psi=\frac{g \beta}{6 \alpha} \frac{\left(T-T_{E}\right)\left(C_{E}-C\right)\left(\rho-\rho_{E}\right)}{G^{2}} \propto\left(C_{E}-C_{L}\right)^{3}, \quad C^{*} \leq C \leq C_{E}
\end{aligned}
$$

The maximum freckle potential in a given binary alloy system with limited solid solubility occurs at the solubility limit $\left(C^{*}\right)$, as schematically shown in Figure 1-6. The preceding analysis is based on driving force only and can be applied to alloy systems in which the solute element can be either lighter or heavier than the matrix-forming elements.

\subsubsection{Freckling in Directional Solidification (DS)}

During directional solidification, the upper region of the mushy zone is believed to provide the favorable conditions for freckle initiation. Different initiation modes have been proposed. Once the plume develops the fluid flow pattern could be such that cold soluterich liquid flows into hotter regions relatively depleted in solute under the influence of gravity. In an attempt to attain solutal equilibrium this liquid would dissolve the surrounding (solute depleted) dendrite arms, thus decreasing the resistance to further flow and ultimately producing a channel.

One of the initiation theories $[22,24]$ states that a small perturbation at the top layer between the mushy zone and the bulk liquid triggers the fluid flow in the system, as shown in Figure 1-7. According to Figure 1-5, a buoyancy term results when the density gradient changes sign at the growth front. However, study by Song and Tewari [23] indicates that macrosegregation along the length of the directionally solidified specimens is produced only when there is convection of interdendritic liquid in the mushy zone and mixing in the bulk liquid. Convection in the bulk melt alone can not produce longitudinal macrosegregation such as channel segregates. 
The sustained translation of liquid vertically against the gravitational field depends on there being retained buoyancy and therefore on how rapidly heat and matter exchange between the moving liquid and surroundings. The ratio between thermal diffusivity, $\kappa$, and liquid diffusion coefficient, $D_{L}$, can be described by Lewis number, $\tau=\kappa / D_{L}$, having values about $10^{3}$ for metals [21]. Thus heat redistribution is rapid and the buoyancy is sustained by composition difference.

\subsubsection{Freckling in Remelting}

The extrapolation of the composition-driven convection to configurations other than vertical directional solidification has been cautioned. The heat flow direction changes gradually from parallel to perpendicular to gravity during remelting of superalloys, which complicates the liquid flow pattern. Multiple solute elements are either rejected into or depleted from the interdendritic liquid in the temperature range of $1200^{\circ} \mathrm{C} \sim 1400^{\circ} \mathrm{C}$. It is difficult to determine the liquid density at such high temperatures experimentally. Hence, the density of liquid has been assumed to be heavier or lighter than surroundings depending on alloying elements.

Mehrabian and Flemings [13] proposed the interdendritic fluid flow pattern in alloys with heavy solute elements under ESR conditions. As shown in Figure 1-8, if the liquid flow lines fan outward, as they would when liquid density is constant, segregation becomes negative. If they fan inward, as they can do as a result of fissuring or liquid density that increases during solidification, positive channel-type segregates occur. A more detailed mechanism of freckle formation in remelt alloy 718 has been proposed recently [25]. In alloy 718, the partitioning of $\mathrm{Nb}$ evidently results in an increase of liquid density during solidification. When the interdendritic liquid flows into a higher temperature field, the liquid composition tends to decrease by remelting some of the solute-lean dendrites in surrounding. This dissolution process provides the basis of the mechanism by which the channel defects form and propagate.

However, it has been argued by Auburtin and Mitchell [26] that minor alloying additions such as $\mathrm{C}, \mathrm{Si}, \mathrm{B}$ and $\mathrm{Zr}$, which are all strong positive segregating elements, could contribute to density inversion for freckling in heavy solutes containing alloys. Heavy 
solute elements were removed from the interdendritic liquid by the formation of carbides. Freckled regions are found to contain vast amount of carbides and these particles do not contribute to the liquid density.

These freckling mechanisms are each supported by different experimental evidence. The dilemma to quantify the effects of solute elements on liquid density needs to be solved in order to establish an acceptable freckling mode in remelting of superalloys.

\subsubsection{Controlling Factors}

Three vital factors - casting geometry, alloy composition, and processing conditions control the freckling tendency in solidification processing of Ni-based superalloys.

The effect of ingot size on freckling was first demonstrated in directionally solidified Udimet-700 [5]. In that case, the average number of freckles is proportional to the ingot cross section area (or volume for fixed ingot height). A critical diameter is required to generate one freckle line.

Remelting of $\mathrm{Nb}$-containing superalloys has long been restricted to a certain size of ingot due to the segregating tendency. The effect is attributed to the sluggish heat transfer in large size ingots, which significantly increases the dendrite arm spacing so as to increase the fluidity of the interdendritic liquid. The size of casting also determines the location of freckles based on the argument that freckles require a certain slope in the growth front in order to develop.

Control and optimization of processing conditions have been proved to be effective to improve the ingot quality of superalloys $[8,27]$. First, steady-state melting environment must be achieved so as to provide a steady supply of liquid metal of constant composition to the solidification zone. Any abrupt variation in the solidification process that results in significant perturbation of the flow fields in the mushy zone will give rise to solute redistribution and hence, macrosegregation. Steady-state solidification depends on arc behavior, magnetic field, clean electrode, etc. [28]. Second, the melt rate and cooling rate must be matched for a given material and ingot size to maintain a relatively shallow pool of constant depth. This becomes increasingly difficult for large ingot due to the poor heat transfer. Finally, there are transients, namely start-up and hot-top, in each remelt process,

which are also critical in segregation control. A successful control requires comprehensive 
process knowledge be integrated into the control program that describe all relevant dynamics of the melting and solidification processes.

The effect of alloy composition on freckling in superalloys has been manifested by remelt processing of alloys 718 and 706 . The chemistry of alloy 706 is similar to alloy 718 $(5 \mathrm{wt} . \% \mathrm{Nb}$ ) except the $\mathrm{Nb}$ content is intentionally reduced to about $3 \mathrm{wt} \%$. This feature leads to the melting of $914 \mathrm{~mm}$ (36 inch) 706 ingot, while alloy 718 is limited to $508 \mathrm{~mm}$ (20 inch) under the same process conditions [2]. Current freckling theories in remelt process are based on the assumption that heavier (as compared with $\mathrm{Ni}$ ) solute elements increase liquid density, while lighter elements decrease liquid density during solidification. The assumption lacks experimental and theoretical basis. Also there are other factors that may depend on composition, such as the flow resistance of the dendritic region. Composition effect on freckling will be reviewed in detail in the section 1.5.

\subsection{Freckling Criteria}

Several freckling criteria have been suggested in the literature and are categorized as local solidification time (LST), Flemings' criteria, and Rayleigh number.

\subsubsection{Local Solidification Time (LST)}

From technology standpoint, it is desirable to define a processing window beyond which freckles will form in the casting. A variety of straightforward mathematical criteria based on the measurable processing parameters such as the temperature gradient, $G\left({ }^{\circ} \mathrm{C} / \mathrm{cm}\right)$, and the solidification rate, $R(\mathrm{~cm} / \mathrm{min})$, have been suggested.

To describe the effect of growth rate on freckling, Copley et al [6] proposed that freckles occur when the local solidification time, $L S T$, exceeds a critical value, $L S T^{*}$.

$$
L S T=\frac{\text { Liquidus }- \text { Solidus }}{G \cdot R}>L S T \text { * }
$$

Liquidus is the temperature at which the liquid alloy begins to solidify, while solidus is the temperature at which the solidification ends. This criterion holds true in both directional solidification and remelting processed superalloys. Yu [8] demonstrated that by improving the ingot heat extraction, local solidification time is decreased, which results in finer 
dendrite arm spacing. These operational parameters are often correlated with dendrite arm spacing that is also important in defining freckling behavior. Pollock [29] studied single crystal superalloys where primary dendrite arm spacing, $d_{1} \propto G^{-1 / 2} \cdot R^{-1 / 4}$, and a critical $d_{1}$ best defines the onset of grain defect formation. A similar relation for secondary dendrite arm spacing, $d_{2}=33.85 \cdot(G \cdot R)^{-0.338}$, in $\mathrm{mm}$, was given in remelting alloy 718 [8]. These are rationalized by the fact that the resistance to liquid flow is reduced when the interdendritic spacing gets larger.

Mori and Ogi [30] studied unidirectional solidified Al-Mg and Al-Mg-Cu alloys in both vertical and horizontal orientations. They found that channel-type segregation form when the solidification rate, $R$, is below a critical value, $R_{C}$.

$$
R_{C}=A \cdot\left(\Delta \rho / \Delta T_{L}\right)^{0.6} \cdot(\sin \theta)^{1.3}
$$

$\Delta \rho$ is the density change of interdendritic liquid over a temperature change of $\Delta T_{L}$ and $\theta$ is the inclination angle of the solidification interface to the horizontal plane. $A$ is constant.

These criteria offer relatively accurate predictions in directional solidified castings and have been adopted in a number of numerical models to simulate freckle formation due to thermosolutal convection. However, they do not account for the effect of alloy chemistry.

\subsubsection{Flemings' Criterion}

Flemings and co-workers [13] considered macroscopically a unit volume in mushy zone in which mass exchange is only realized by liquid flow with surrounding and freckling criterion is:

$$
\frac{\vec{V} \cdot \nabla T}{\varepsilon}<-1
$$

Where $\vec{V}$ is the velocity of the interdendritic liquid, $\nabla T$ is the temperature gradient, which is usually denoted as $G$ in unidirectional solidification. $\varepsilon$ is the cooling rate, which is the product of $G$ and $R$ under directional solidification condition. The physical meaning of the criterion is that freckles will form when the interdendritic liquid flow velocity aligned in the direction of crystal growth is greater than the solid growth rate. The equation is difficult to apply due to the implicit form and it is improved to explicit form in chapter 5 . 


\subsubsection{Rayleigh Number}

The buoyancy-driven convection tendency was first formulated by Rayleigh and refined by Jeffries and the thermal Rayleigh number takes the following format [22],

$$
R_{T}=\frac{g \rho \alpha(d T / d z) h^{4}}{\eta \kappa}
$$

Where is $\rho$ the liquid density of the alloy, $\alpha$ is the thermal coefficient of volume expansion, and $d T / d z$ is the temperature gradient. The term in equation (1-6) is dimensionless and the combination $h^{4} / \eta \kappa$ has reciprocal units with the buoyancy term. The equivalent solutal version of it,

$$
R_{S}=\frac{g \rho \beta(d C / d z) h^{4}}{\eta D_{L}}
$$

Where is $\alpha$ is the thermal coefficient of volume expansion, and $d C / d z$ is the composition gradient. Since in vertical directional solidification, the thermal gradients are positive and stabilizing, it is of interest to consider the solute effect on the instability. The Rayleigh number becomes significant when the buoyancy term equal or exceeds the inertia of the system. Its value is sensitive to the selection of characteristic length of the system, $h$. Typical characteristic lengths used in literature are 1) primary dendrite arm spacing; 2) mushy zone depth.

Sarazin and Hellawell [22] treated liquid disturbance close to the dendritic front, having a periodicity determined by the primary arm spacing. Thus they used that spacing as characteristic for the initial perturbation. However, Pollock [7] pointed out that dendrite fragmentation also contributes to grain defect formation in superalloys and it is likely that the permeability of the dendritic network influences the criteria, thus the mushy zone depth is used as $h$. For porous media, $h^{4}$ is reduced to $K h^{2}$, where $K$ is the permeability parallel to primary arms. Following the Kurz and Fisher [31], the outer envelope of the dendrite arm can be approximated by $r=\lambda^{2} / 3 h$, where $h$ is depth of mushy zone. Also, since $d C / d z=G / m_{L}, m_{L}$ is the liquidus slope, 


$$
R_{S}=\frac{g \beta G}{9 r^{2} \eta m_{L}}\left(\frac{K \lambda_{1}^{4}}{D_{L}}\right)
$$

It is clear that the development of convection-induced freckle defects will be enhanced in high permeability dendrite networks with large dendrite arm spacing. If the arm spacing is replaced by $G^{-1 / 2} \cdot R^{-1 / 4}$, then for a given alloy system, $R_{S}$ becomes proportional to $1 / G R$. Therefore Rayleigh criterion and the cooling rate criterion are similar and $R_{S}$ is also proportional to the local solidification time.

Rayleigh number is the most complete criterion available, which combines two of the three controlling factors: alloy chemistry and processing conditions, but not casting geometry. Example of using $1 / R$ as characteristic length will be given in chapter 5 .

\subsection{Composition Effects}

In any dendritic solidification, Rayleigh number can be written as [26]:

$$
R a=f\left(\eta, D_{L}, D_{T}, d \rho / d z, K\right) \times g\left(G, R, d_{1}, d_{2}, L S T\right)
$$

The most researched factor is the "casting conditions factor" ( $g$ function) because it provides insights for the reduction of freckle defects via process control. The "composition factor" ( $f$ function) describes the composition effect in a given alloy with varied solute content or identical castings of different alloys. The composition factor is not known to great precision and some of the properties are often artificially rectified to account for a critical $R a$ threshold. This section summarizes the present understanding of composition effects on freckling by reviewing microsegregation theory and its strong correlation with macroscopic segregation phenomena. Results from literature are categorized into binary and superalloy systems.

\subsubsection{Solute Redistribution in Dendritic Solidification}

In dendritic solidification, solute redistribution (microsegregation) occurs in such a way that substantial differences in alloy content result over distances the order of the dendrite arm spacing. The classical quantitative treatment of solute redistribution has been derived initially by Gulliver, Scheil, and Sfann, then improved by Flemings [32]. For a small volume element inside mushy zone, the Scheil equation is written: 


$$
C_{S}^{\wedge}=k C_{0}\left(1-f_{S}\right)^{k-1}
$$

Where $C_{S}^{\wedge}$ is the interface composition of the solid when the fraction of solid within the volume element is $f_{S}, C_{0}$ is the initial alloy composition within the volume element. $k$ is the equilibrium partition ratio between $C_{S}^{\wedge}$ and the liquid composition $C_{L}, k=C_{S}^{\wedge} / C_{L}$. The partition ratio is dependent on temperature and alloy composition. The detailed formulation of equation (1-10) can be found in Appendix A, in which $k$ is assumed to be constant. Solidification begins in the volume element and proceeds with a flat solid/liquid interface. Negligible solid diffusion and complete liquid diffusion are assumed. Liquid composition is:

$$
C_{L}=C_{0}\left(1-f_{S}\right)^{k-1}=C_{0} f_{L}^{k-1}, \quad f_{L}=1-f_{S}
$$

When a temperature gradient exists within a solidifying casting, gradient in fraction solid and liquid composition must also exist. Macrosegregation depends on the way in which liquid composition and/or fraction solid vary with temperature. For phase diagrams whose liquidus is a straight line, the liquid composition with the volume element is simply related to temperature by:

$$
C_{L}=\frac{T-T_{M}}{m_{L}}
$$

where $T$ is the temperature of the volume element, $T_{M}$ is the melting point of pure solvent, $m_{L}$ is the liquidus slope and $m_{L}=d T / d C$. For an alloy $C_{0}$ with liquidus of $T_{0}$, the dependency of fraction liquid in the volume element on temperature is

$$
f_{L}=\left(1+\frac{T-T_{0}}{m_{L} \cdot C_{0}}\right)^{1 /(k-1)} \text { Or } T=T_{M}+m_{L} \cdot C_{0} \cdot f_{L}^{(k-1)}
$$

In Scheil model, $C_{0}, m_{L}$, and $k$ are the key material parameters that affect the solute redistribution. The only factor that is not considered in the model is the eutectic temperature $T_{E}$, or in some cases, the solidus temperature $T_{S}$, which determines the temperature range of solidification:

$$
\Delta T=T_{L}-T_{E}=T_{L}-T_{S}
$$

The liquid density change with the mushy zone is dependent on $\Delta T$ and $\Delta C_{L}$. For binary alloys with limited solubility $C^{*}$ as shown in Figure 1-6, both $\Delta T$ and $\Delta C_{L}$ reach 
maximum value at $C^{*}$. This coincides with Copley's freckle potential theory and will be used to design freckle-prone alloys.

\subsubsection{Analog Systems}

Analog systems include non-metallic aqueous system, $\mathrm{NH}_{4} \mathrm{Cl}-\mathrm{H}_{2} \mathrm{O}$, and low melting-point metallic systems, such as $\mathrm{Al}-\mathrm{Cu}, \mathrm{Al}-\mathrm{Mg}$, and $\mathrm{Pb}-\mathrm{Sn}$.

Sarazin and Hellawell [22] studied channel formation in $\mathrm{Pb}-\mathrm{Sn}, \mathrm{Pb}-\mathrm{Sb}, \mathrm{Pb}-\mathrm{Sn}-\mathrm{Sb}$ alloys in which solute is less dense than solvent and the liquid density inside mushy zone is positive (density inversion). Effects of solute concentration on freckling are summarized as follows. 1) Lower concentration limits exist below which channels do not form. In the $\mathrm{Pb}-\mathrm{Sn}$ system lower limit lies between 2.5 and 5.0 wt pet $\mathrm{Sn}$ and in the $\mathrm{Pb}-\mathrm{Sb}$ system between 1.0 and $2.0 \mathrm{wt}$ pct Sb. Channels are narrow and sharply defined at lower composition ranges. 2) There is no sharply defined upper limit for channel formation. Channels become diffuse, of irregular section, and confined to the center of the specimen as the concentration increases. The information is summarized in Figure 1-9. The principle change with composition is an increasingly open or permeable dendritic mesh, as a fraction of liquid within the array arises (without significant change in the primary dendrite spacing. Therefore, as the fraction of liquid increases, liquid entrapment becomes more efficient and the circulation allows broader channels to develop. There are both lower and upper limits to the dendritic permeability, which allow such circulation patterns to become stabilized and maintained.

A similar behavior has been seen in Ni-Al binary alloys [5]. These alloys (Al solubility $=21 \mathrm{wt}$ pct) were cast under conditions suitable for single crystal growth. At 1 and 5 wt pct Al no freckles are evident. Spurious nucleation, caused by relative local cooling conditions, did result in large grains rather than single crystals. At the level of 8 wt pct $\mathrm{Al}$ a few freckle grains can be noted and at 10 wt pct $\mathrm{Al}$ the structure is freckled profusely.

\subsubsection{Ni-based Superalloys}

Ni-based superalloys are multicomponent alloys in which more than one solute element is added to the matrix for strengthening, oxidation and corrosion resistance, and other 
purposes. A list of superalloys that have been reported in literature for freckling using DS or remelt process is given in Table 1-1. These alloys contain various levels of $\mathrm{Al}, \mathrm{Ti}, \mathrm{Nb}$, Mo, and $\mathrm{W}$ as solute elements [2, 5, 33-35].

Table 1-1: Chemical compositions of alloys reported for freckling (wt pct)

\begin{tabular}{cccccccccccc}
\hline Alloys & $\mathrm{Ni}$ & $\mathrm{Cr}$ & $\mathrm{Fe}$ & $\mathrm{Co}$ & $\mathrm{Mo}$ & $\mathrm{W}$ & $\mathrm{Nb}$ & $\mathrm{Ti}$ & $\mathrm{Al}$ & $\mathrm{C}$ & $\mathrm{Si}$ \\
\hline 718 & Bal. & 18 & 18 & - & 3 & - & 5.4 & 1 & 0.5 & 0.025 & 0.2 \\
706 & Bal. & 16 & 38 & - & - & - & 3 & 1.75 & 0.25 & 0.015 & 0.2 \\
625 & Bal. & 22 & 4.4 & - & 9.1 & - & 3.53 & 0.3 & 0.2 & 0.02 & 0.2 \\
Waspaloy & Bal. & 20 & - & 13 & 4.25 & - & - & 3 & 1.3 & 0.035 & - \\
720 & Bal. & 16 & - & 15 & 3 & 1.25 & - & 5 & 2.5 & 0.015 & - \\
Udimet700 & Bal. & 14 & - & 14.75 & 3.8 & - & - & 4.19 & 3.6 & 0.075 & 0.06 \\
Mar-M200 & Bal. & 9 & - & 10.9 & - & 13.2 & 1 & 2.3 & 4.9 & 0.14 & 0.07 \\
A-286 & 25.5 & 14.7 & Bal. & - & 1.25 & $0.3 \mathrm{~V}$ & $1.5 \mathrm{Mn}$ & 2.1 & 0.2 & 0.02 & 0.7 \\
\hline
\end{tabular}

No phase diagram is available for these complex alloy systems and the majority of solidification studies rely on experimental and empirical approaches. Material parameters such as liquidus slope, liquidus temperature, and partition ratio differ from those obtained in respective binary systems due solute interactions. An element having partition ratio less than unity will be rejected into interdendritic liquid during solidification, such as $\mathrm{Ti}$ and $\mathrm{Nb}$. The matrix forming elements, such as $\mathrm{Ni}, \mathrm{Cr}, \mathrm{Fe}$, and $\mathrm{Co}$, all have partition ratios close to one. The partition ratios in superalloys have been summarized by Sung [36].

Experimental alloys with factorial designed composition variations are often used to investigate microsegregation behaviors. The contribution of refractory alloying additions, Re, Ta, and $\mathrm{W}$ to the breakdown of solidification of single crystal superalloys was studied [7, 29]. It was found that higher level of Ta is effective in suppressing the formation of both freckles and columnar grains, while the effects of Re and $\mathrm{W}$ are weaker. No correlation between defect formation and the solidus, liquidus, or freezing ranges of the alloys was apparent. Another example is a series of Ni-Cr-Fe-Nb alloys with variation of $\mathrm{Cr}, \mathrm{Fe}$, and $\mathrm{Nb}$ [37]. Results indicate that a high level of $\mathrm{Cr}$ (20 wt pct) together with certain amount of Fe (10 wt pct or above) will cause the formation of Laves in eutectic. Laves can be avoided if $\mathrm{Cr}$ addition is reduced to 12 wt pct. Replacement of Fe with an optimum level of Co also eliminates Laves formation. These studies provide valuable 
insights to understand real behavior in commercial alloys. However, they are often timeconsuming and restricted by the availability of resources.

Thermodynamic calculation of multicomponent phase diagrams using the CALPHAD (CALculation of PHAse Diagrams) method has been proved to be a successful method to reduce the amount of experimental work in research and development. Preliminary results in solidification simulation exhibit reasonable agreement with experiments $[38,39]$. The approach will be explored in the present research to study the solidification segregation in model alloys.

\subsection{Objectives of Research}

Freckles are casting defects that form in superalloys as result of the thermosolutal convection of interdendritic liquid during solidification. Experience in directional solidification shows that the fluid flow is driven by density inversion in the mushy zone. However, the theory has difficulty to explain the freckling phenomena in remelting of superalloys due to the distinctive differences in processes and alloys. Freckle occurrence is known to be dependent on alloy chemistry, casting geometry and casting conditions. Freckle formation has been avoided in ingot production by carefully controlling the processing conditions. However, as gas turbine industry calls for more elaborate alloying additions, the process seems to have reached certain limits. No systematic study is available to provide a definite assessment of the alloying effect on freckle formation. Based on the literature review, the objectives of this research work are proposed:

- Understand the freckling mechanism in remelt processing of Ni-based superalloys. In particular, the density inversion theory will be verified.

- Identify key controlling parameters of freckling in remelting process superalloys.

- Determine composition effect on the key controlling parameters by (1) investigating solidification behavior in model alloy systems using advanced thermodynamic simulation approach; (2) experimentally determine the key controlling parameters of model alloys.

- Develop freckling index based on composition effects. 

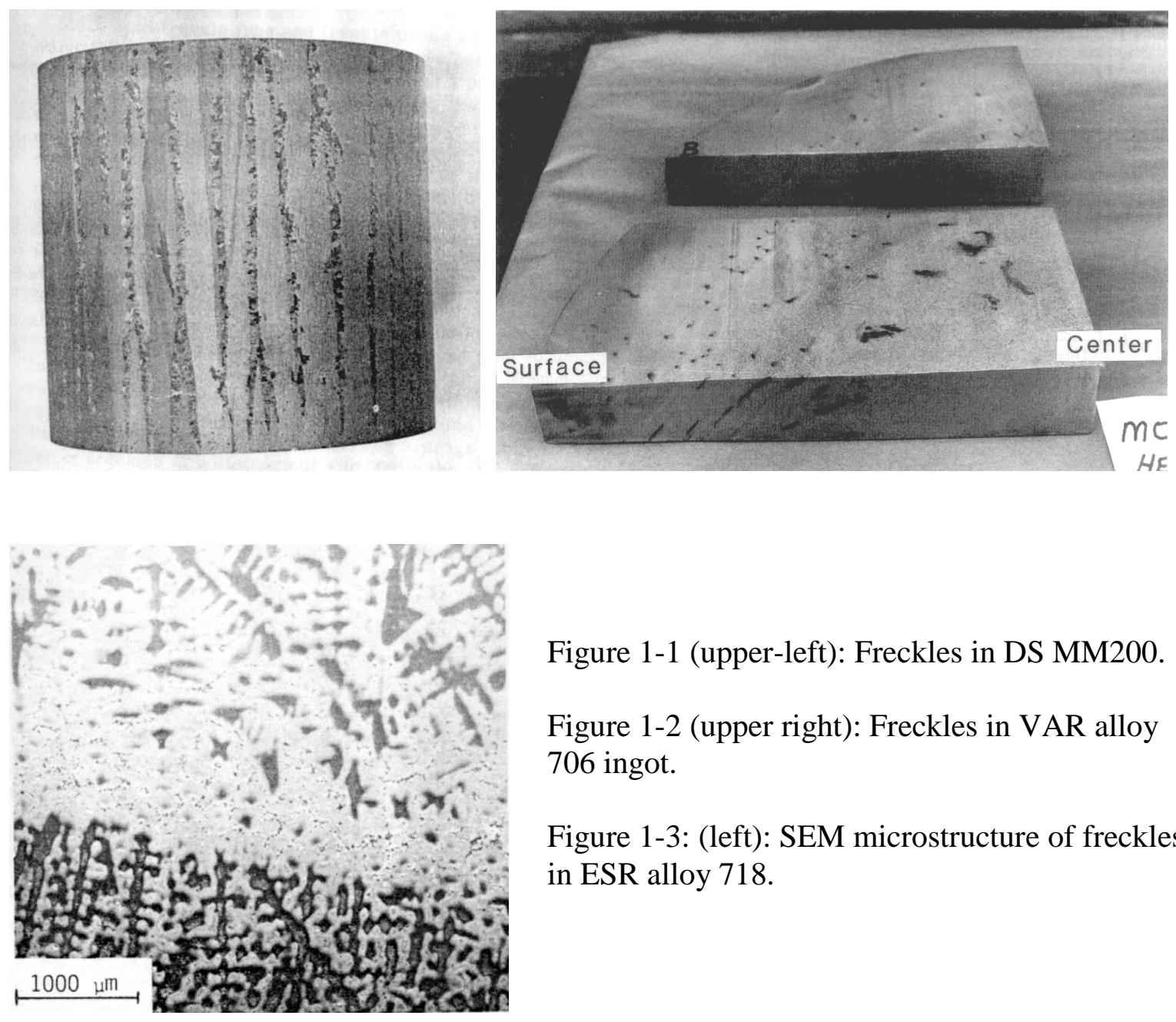

Figure 1-1 (upper-left): Freckles in DS MM200.

Figure 1-2 (upper right): Freckles in VAR alloy 706 ingot.

Figure 1-3: (left): SEM microstructure of freckles in ESR alloy 718.

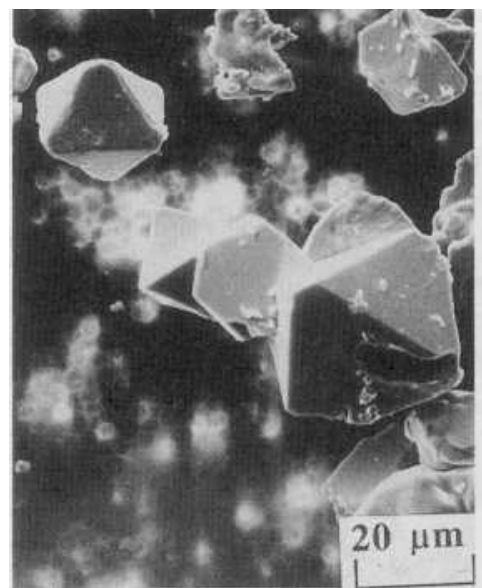

(a)

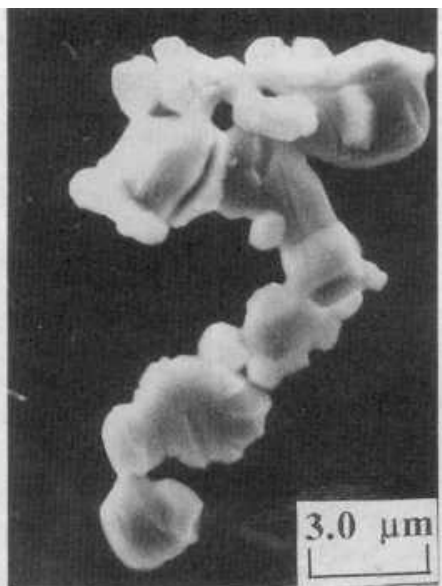

(b)

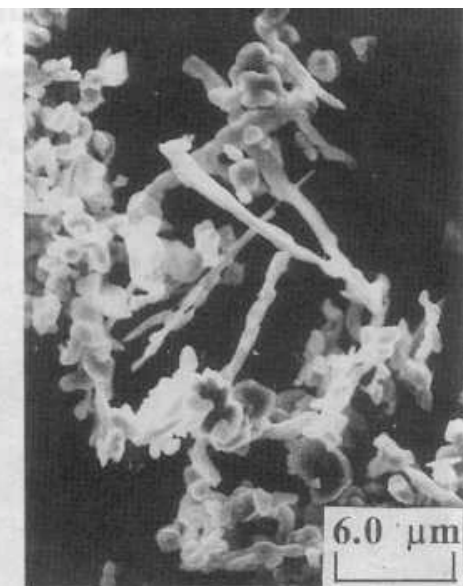

(c)

Figure 1-4: SEM of extracted phases from freckled area. (a) NbC carbide; (b) Laves; (c) $\eta$. 


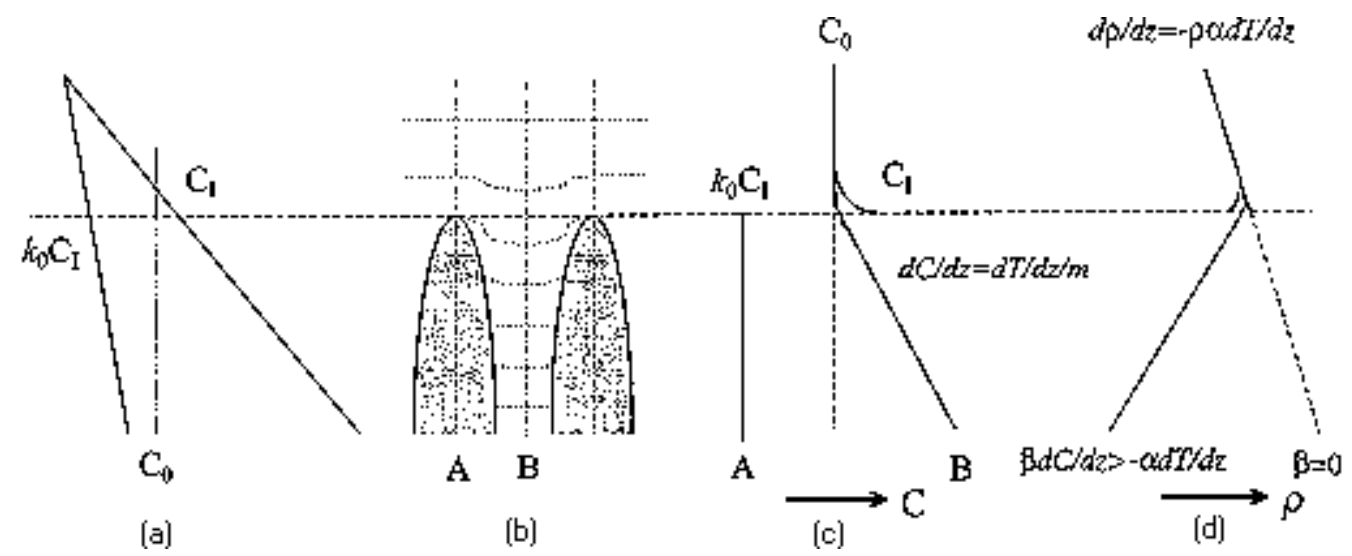

Figure 1-5: (c) Composition and (b) density profiles through (b) an upward growing dendrite. (a) Trace A along a dendrite axis and trace B, interdendritic, with reference to a phase diagram (a).

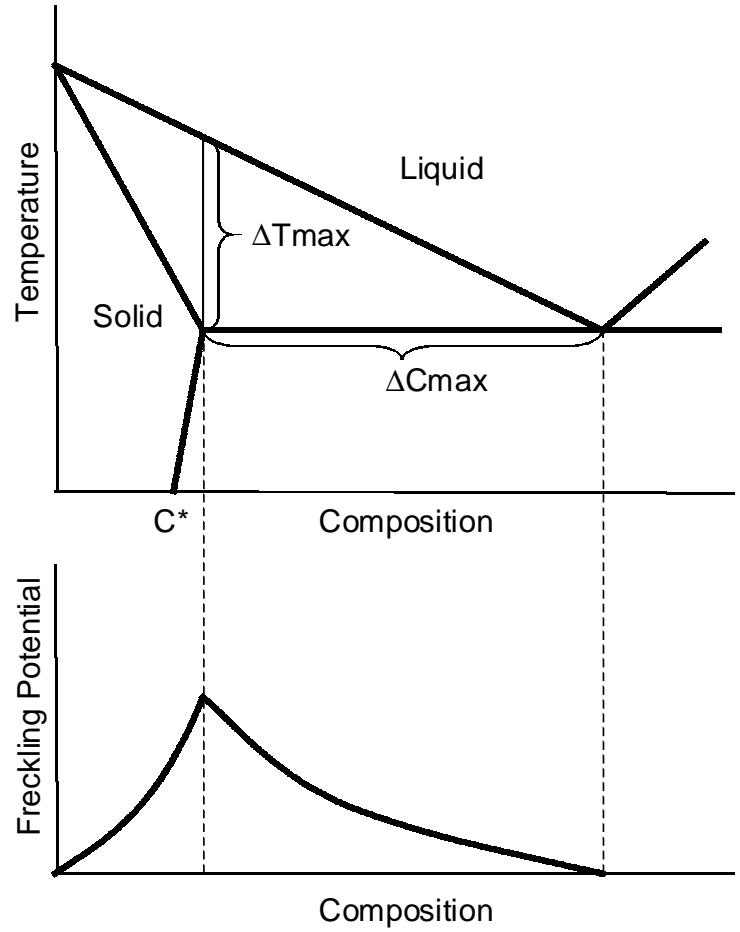

Figure 1-6: (top) A phase diagram with limited solid solubility, $\mathrm{C}^{*}$; (bottom) the dependence of freckle potential on composition. $[a]$

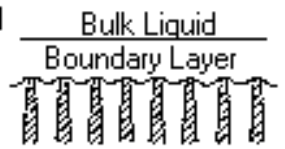

(b)

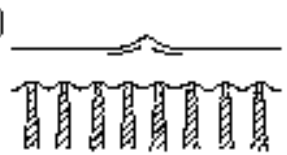

$[\mathrm{c}]$
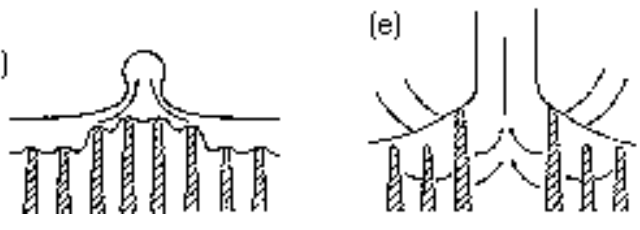

Figure 1-7: Schematic showing the density inversion theory and interface perturbation. 


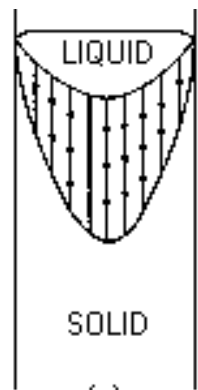

[a]

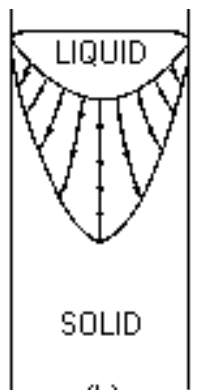

$[b]$

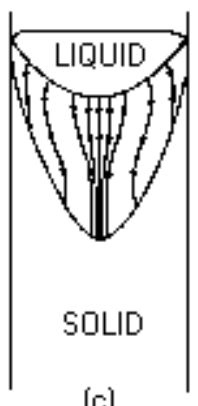

[c]

Figure 1-8: Interdendritic flow pattern in remelting by Mehrabian [13].

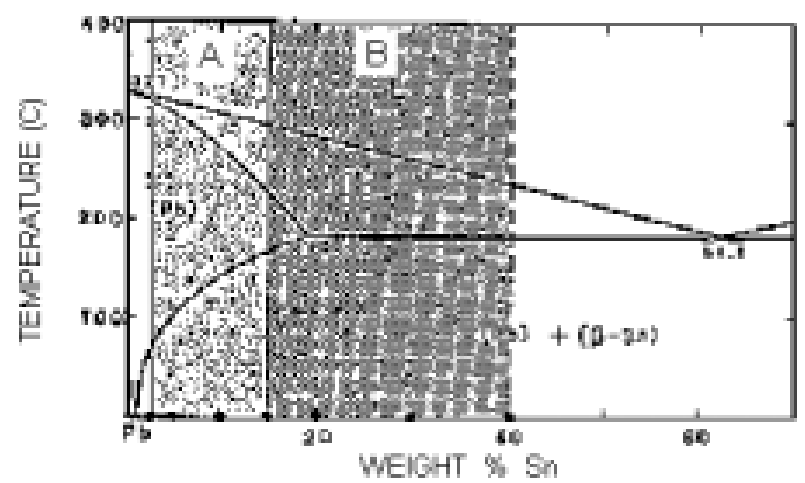

A. Mutiple Smal Channels

(a)

B: Single Large Channel

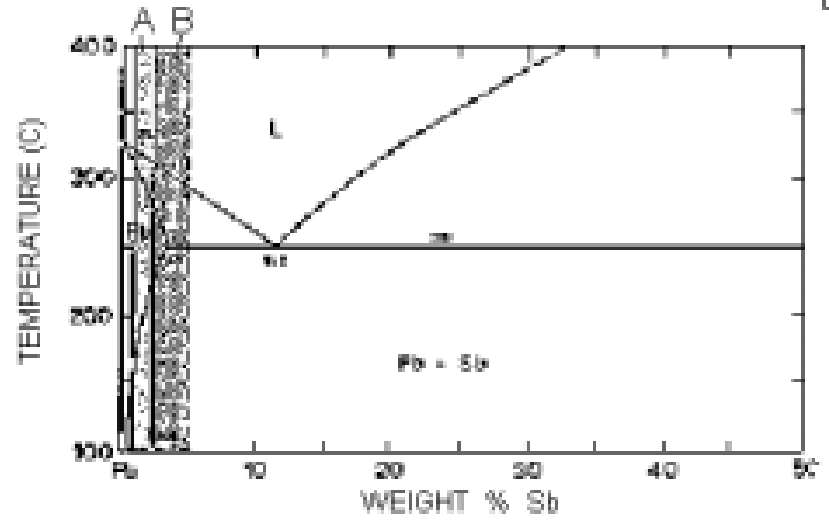

(b)

Figure 1-9: Composition effects on freckling in $\mathrm{Pb}-\mathrm{Sb}$ and $\mathrm{Pb}-\mathrm{Sn}$ binary systems. 


\section{Chapter 2}

\section{Experimental}

2.1. Introduction 20

2.2. Alloy Selection 20

2.2.1. Model Alloys 20

2.2.2. Industrial Alloys 21

2.3. Horizontal Solidification 21

2.4. Differential Thermal Analysis 22

2.5. Metallography 23

2.5.1. Sample Preparation 23

2.5.2. Optical Microscope 23

2.5.3. Scanning Electron Microscope 23

2.5.4. Energy Dispersive Spectrometry 24

2.6. CALPHAD 24

2.6.1. Thermo-Calc ${ }^{\mathrm{TM}} 24$

2.6.2. NiFe-Database 25

2.7. Liquid Density Calculation 26

$\begin{array}{lll}2.8 . & \text { Summary } & 27\end{array}$ 


\subsection{Introduction}

The purpose of this chapter is to introduce the experimental and theoretical techniques that are used for the determination of various quantities of solidification in the current study. It describes the basic features and theories of the techniques. Details that are closely related to the current research are also included in the specific chapters to follow. It is not intended to contain all the details of methods and please refer to requisite references if more information is needed.

\subsection{Alloy Selection}

\subsubsection{Model Alloys}

Three model alloys are designed to verify the density inversion theory in superalloys. They are Ni-rich alloys with 20 wt pct $\mathrm{Cr}$ to form face-centered-cubic primary solid solution, $\gamma$. A direct comparison is made between composition with addition of Ti, whose atomic weight is lighter than $\mathrm{Ni}$ and will cause density inversion, and compositions with additions of $\mathrm{Nb}$, which assimilate commercial $\mathrm{Nb}$-containing superalloys that showed freckles in VAR or ESR ingots. Levels of $\mathrm{Ti}$ and $\mathrm{Nb}$ are selected to be slightly lower than their solubility limits at which the freckling potentials reach to maximum according to Copley (see Chapter 1 section 1.3.). The chemical compositions are given in Table 2-1. Figure 2-1 shows the isopleth at 20 wt pet $\mathrm{Cr}$ of the $\mathrm{Ni}-\mathrm{Cr}-\mathrm{Ti}, \mathrm{Ni}-\mathrm{Cr}-\mathrm{Nb}$ and $\mathrm{Ni}-\mathrm{Cr}-\mathrm{Fe}-\mathrm{Nb}$ (18 wt pct Fe) systems.

The alloys were prepared at Technology Processing Center (TPC), Special Metals Corporation at Huntington, WV, using vacuum induction melting as $22 \mathrm{~kg}$ (50 lbs) and 100 mm (4 inch) diameter cylindrical ingots. These ingots are designated as HV8851, HV8852, and HV8853. Levels of trace elements, such as $\mathrm{C}$ and $\mathrm{Si}$, were reduced during melting to minimize their effects on segregation. Samples were taken from the top sections of the ingots for metallography and differential thermal analysis (DTA). The same composition designs were used in the horizontal solidification at Daido Steel Corporation, Japan. The method is described in section 2.3 and the compositions are also given in Table 2-1. These ingots are designated as RN902, RN903, and RN904. 
Three series of Ni-based model alloys were prepared in a previous project [40] to investigate the alloying effects of $\mathrm{Nb}, \mathrm{Ti}$ and $\mathrm{Al}$ on terminal phases. $\mathrm{Ti} / \mathrm{Nb}$ ratio (atomic pct) varies at levels of $1,1.5$, and 2 in the following alloy systems: 1) Ni-20Cr-18Fe-Nb$\mathrm{Ti}$; 2) Ni-20Cr-18Fe-Nb-Ti-1Al; 3) Ni-20Cr-36Fe-Nb-Ti. It was revealed that three secondary phases, Laves phase, $\delta-\mathrm{Ni}_{3} \mathrm{Nb}$, and $\eta-\mathrm{Ni}_{3} \mathrm{Ti}$, form during eutectic reaction. These alloys will be used in the present study to investigate the influence of the composition variables on freckling tendency. The alloys were prepared using similar procedure as stated in previous paragraph. The actual compositions are given in Table 2-1.

\subsubsection{Industrial Alloys}

Industrial superalloys 718, 706 and 625 are selected in the current research. All of the three alloys contain certain levels of $\mathrm{Nb}$ that is heavier than Ni. Alloy 718 also has small amounts of $\mathrm{Ti}$ and Al. Shop experience indicates that these alloys are freckle-prone in VAR or ESR when the ingot size exceeds certain limit. In this study, alloy 718 was processed by VAR at Special Metals Corporation and the samples were obtained in as-cast condition. Alloy 706 was processed by ESR. Freckles were found in an alloy 625 billet, which was originally processed using VAR and forged to billet. Specimens of 625 were taken from both freckled and non-freckled areas. Table 2-2 lists the chemical compositions of the selected alloys.

\subsection{Horizontal Solidification}

In VAR or ESR, the norm of growth fronts gradually changes from vertical to horizontal orientations. There is always a horizontal component of solidification, where the probability of channel segregation is the highest. Horizontal solidification is analogous to solidification from sidewall of a large ingot; therefore it can be used as physical simulation of freckle formation in remelting under controlled experimental conditions.

The schematic of the horizontal solidification setup is shown in Figure 2-2. The crucible is of rectangular shape with a dimension of $220 \mathrm{~mm}$ (height) by $140 \mathrm{~mm}$ (length) by $30 \mathrm{~mm}$ (width). It is made of two layers of high purity alumina (99.8\%) to prevent from cracking during solidification. The crucible is heated from three sides and cooled from one 
side. The cooling is realized by using an alumina chill block at one side of crucible with argon flowing in and out of it. Alloys are melted in an induction furnace and poured at around $1500^{\circ} \mathrm{C}$ into the crucible which is pre-heated at $1100 \sim 1200^{\circ} \mathrm{C}$. Unidirectional solidification is controlled by changing the argon flow rate and monitored by thermocouples inserted into the ingot at about $20 \mathrm{~mm}$ intervals along the growth direction.

Horizontal solidification of the three model alloys (Ni-20Cr-6Ti, Ni-20Cr-8.4Nb, and $\mathrm{Ni}-20 \mathrm{Cr}-18 \mathrm{Fe}-7 \mathrm{Nb}$ ) was carried out at the research laboratory of Daido Steel Corporation in Japan. Ingots were sectioned in the longitudinal plane for preliminary macrostructure examination and then shipped to US for further analysis.

\subsection{Differential Thermal Analysis}

Differential Thermal Analysis (DTA) has been widely utilized to simply and accurately measure the temperatures of phase reactions in materials, as well as to obtain small as-cast samples with controlled cooling rate. It measures the temperature difference, $\Delta T^{*}$, between a small sample of the metal to be characterized and a transformation free reference material undergoing the same thermal cycle. During solidification process, heat release by phase transformation taking place in the sample induces peaks to appear on the temperature difference line, which would be otherwise perfectly flat, provided that the weights of sample and reference have been properly matched.

TA1600 DTA cell is used in the current study. Test specimens weighed from $200 \mathrm{mg}$ to $300 \mathrm{mg}$ and were measured using a precision balance before testing. Pure Ni is used as the reference material because its melting point $\left(1450^{\circ} \mathrm{C}\right)$ is higher than that of most superalloys and it is transformation free at test temperatures up to $1420^{\circ} \mathrm{C}$. Another advantage of using pure Ni reference is that its specific heat is similar to Ni-based alloys so that a relatively flat baseline of temperature difference can be obtained. The DTA cell was calibrated to within $\pm 2^{\circ} \mathrm{C}$ for both upper and lower temperature limits using pure silver and Ni. Samples were heated to $900^{\circ} \mathrm{C}$ at $100^{\circ} \mathrm{C} / \mathrm{min}$, then ramp to $1400 \sim 1420^{\circ} \mathrm{C}$ at $20^{\circ} \mathrm{C} / \mathrm{min}$. The furnace was held at the peak temperature for $3 \sim 5 \mathrm{~min}$ before cooling down to $900^{\circ} \mathrm{C}$ at $20^{\circ} \mathrm{C} /$ min. Sample and reference were protected from oxidation by flushing pure argon (99.9995\% purity) through the furnace chamber during testing. 


\subsection{Metallography}

\subsubsection{Sample Preparation}

Samples were grind by using \#240, \#400, \#600, and \#800 grit silicon carbide paper (Buehler). The samples were then polished by using 3 micron diamond paste, and 1 micron and 0.3 micron alumina powder abrasive on imperial cloths. The polishing was completed with a $0.05-\mu \mathrm{m}$ alumina abrasive powder on imperial cloth. Most of the solidified samples were examined in as-polished condition using scanning electron microscope. The freckled samples were etched using appropriate etchant to reveal segregation features.

\subsubsection{Optical Microscopy}

Optical microscopy was performed at Metallography Laboratory, Special Metals Corporation, using LECO optical microscope. Microphotographs were taken at magnifications of $12 \mathrm{x}$ to $100 \mathrm{x}$.

\subsubsection{Scanning Electron Microscopy (SEM)}

SEM analysis was conducted on a JEOL-6400 Scanning Electron Microscope equipped with a fully automated PGT Energy Dispersive Spectrometer. The operating voltage was $20 \mathrm{keV}$ and the current of the condenser lens was $0.3 \mathrm{nA}$. Photomicrographs were taken using both back-scattered electron (BSE) image mode and secondary electron image mode. BSE mode reveals contrast based on atomic weight of the elements, the heavier the element, the brighter is the contrast. Thus it helps to identify dendritic segregation since the interdendritic areas consist of $\mathrm{Nb}$-rich phases such as Laves and $\delta$. X-ray elemental mapping is another powerful tool in SEM to reveal elemental segregation by scanning selected element distribution in the sample.

\subsubsection{Energy Dispersive Spectrometry (EDS)}

EDS utilizes a special detector attached in the SEM chamber to collect characteristic X-ray photons excited from the metal sample by the electron beam. Correction algorithm is used to quantitatively determine the chemical composition by comparing the collected elemental 
intensities with available standards. Multi-element standards and pure element standards were both used in the EDS analysis. It is not the subject of the current study to detail the calculation procedure and please refer other textbooks if interested.

A statistically significant point count technique was incorporated with EDS to obtain the solute profiles in the solidified samples. A 6 by 6 square mesh was used and a computer program automatically carried out the measurements at each node with 100 second collection time. Details of the data processing technique are given in Chapter 6.

\subsection{CALPHAD (CALculation of PHAse Diagrams)}

CALPHAD method is a computation method used to predict the phase behavior of complex, multicomponent materials based on the extrapolation of high-order properties from their lower-order binary and ternary systems. Recently developed databases for superalloys have made the accurate calculation possible for various phase reactions [39]. Several advantages are offered by the method, such as accurate prediction of multicomponent phase reactions and replacement of empirical treatments that would otherwise be used. The method is used in the current study for solidification simulation and segregation study.

\subsubsection{Thermo-Calc ${ }^{\mathrm{TM}}$}

(Thermo-Calc ${ }^{\mathrm{TM}}$ is the trade name of the Division of Computational Thermodynamics, Royal Institute of Technology, Stockholm, Sweden.)

CALPHAD (CALculation of PHAse Diagrams) method calculates phase equilibria through Gibbs energy minimization approach if the Gibbs energies of various phases as functions of temperature and compositions are known. Free energy functions of highly complex, multi-component materials are based on the extrapolation of high-order properties from their lower-order binary and ternary systems which are available in literature. The thermodynamic model used to describe the properties of phases can be represented by a general equation [38]:

$$
\Delta G=\Delta G^{0}+\Delta G_{m i x}^{i d e a l}+\Delta G_{m i x}^{x s}
$$


where $\Delta G^{0}$ is the free energy of the phase in its pure form, $\Delta G_{m i x}^{\text {ideal }}$ is the ideal mixing term and $\Delta G_{m i x}^{x s}$ is the excess free energy of mixing of the components.

The free energy of the substitution model for a multicomponent system is written:

$$
\Delta G=\sum_{i} x_{i} \Delta G_{i}^{0}+R T \sum_{i} x_{i} \ln x_{i}+\sum_{i} \sum_{j>i} x_{i} x_{j} \sum_{v} \Omega_{v}\left(x_{i}-x_{j}\right)^{v}
$$

where $x_{i}$ is the mole fraction of the component $i, \Delta G_{i}^{0}$ defines the free energy of the phase in the pure component $i, T$ is the temperature and $R$ is the gas constant. $\Omega_{v}$ is an interaction coefficient depending on the value of $v$. In practice the value of $v$ lies between 0 and 2. Ternary interactions are often considered but there is little evidence of the need for interaction terms of a higher order than this. Various forms of excessive terms have been considered and reviewed in reference [39].

Once the thermodynamics of the various phases are defined phase equilibria can be calculated using software package such as Thermo-Calc ${ }^{\mathrm{TM}}$ which is the program used in this work. The main module of the program is a Gibbs energy minimization process called Poly-3. Scheil solidification simulation can be calculated by conforming to the local equilibrium assumption. The calculation starts with the nominal composition of the alloy and completely liquid phase in the system. By stepping with small decrements of the temperature, one can determine the new composition of the liquid and then remove the amount of solid phase formed by resetting the overall composition to the new liquid composition before the next step. This procedure naturally treats the appearance of new phases at eutectic reactions or the disappearance of phases at peritectic reactions. The outputs of Scheil module in Thermo-Calc include composition, temperature, and thermodynamic variables such as free energy, entropy, and specific heats, etc.

\subsubsection{NiFe-Database}

$\mathrm{NiFe}$-database is a specially developed database for Ni-based and Fe-based superalloys. It contains the thermodynamic parameters for the description of various phases in superalloys. These parameters are extrapolated from simple binary and ternary constituent diagrams and have been verified extensively using experimental data. During simulation runs, Thermo-Calc uses special subroutines to import the parameters into the calculation 
module. The database used in the current study is version 3 developed by Saunders [39] and includes the following elements: Ni, Cr, Co, Fe, Mo, Nb, Ti, Al, C, B, and N. Among them, $\mathrm{C}$ was not used in any of the simulations of model alloys in the current study.

\subsection{Liquid Density Calculation}

Accurate prediction of liquid density during dendritic solidification is critical in the study of macrosegregation in Ni-based superalloys. Direct measurements of liquid density of transition metals and alloys have been experimentally difficult due to the high melting points of these materials $[41,42]$. Not until recently have people been able to compile the related properties for the calculation of liquid density using theoretical treatment [43].

The basic descriptions of density of pure metal in solid and liquid states are:

$$
\begin{aligned}
& \rho=\rho_{20} \cdot[1+\alpha \cdot(T-20)]^{-3}, \quad T<T_{M} \\
& \rho=\rho_{M}+\frac{d \rho}{d T} \cdot\left(T-T_{M}\right), \quad T>T_{M}
\end{aligned}
$$

where $\alpha$ is the linear expansion coefficient, $\rho_{20}$ and $\rho_{M}$ are densities of pure metal at $20^{\circ} \mathrm{C}$ and melting point $T_{M}$ respectively, $d \rho / d T$ is the temperature coefficient of liquid density. For each element, the density used is the lesser of its solid and liquid densities when extrapolated to the temperature at which the alloy density is calculated $[41,43]$.

The molar volume, $V_{i}$, of the pure element can be calculated using $V_{i}=M_{i} / \rho_{i}$, where $M_{i}$ is the atomic weight of the element. Then the liquid density of an alloy is

$$
\rho=\frac{M}{V}=\frac{\sum_{i} X_{i} M_{i}}{\sum_{i} X_{i} V_{i}+\Delta V^{M}}
$$

where $X i$ is the atomic fraction of the component and $\Delta V^{M}$ is the mixing volume. The mixing volume is independent of temperature and only depends on composition. A simple regression was done to obtain the following equation [43],

$$
\Delta V^{M}=b_{0}+b_{1} X_{1}+b_{2} X_{2}+b_{3} X_{3}
$$

where $X$ 's are summations of atomic fractions of different elements, and $b$ 's are constants. 
METALS, a computer program developed by National Physics Laboratory (NPL, UK), is based on a similar model described above. The model is held to be accurate to about 5\%. After the mixing effect is corrected, the calculation error is reduced to less than $3 \%$ as compared with experimental liquid densities of superalloys. The comparison was made between METALS*'s calculation and experimental data obtained from [43], in which no trace elements $(\mathrm{C}, \mathrm{P}, \mathrm{S}, \mathrm{Si}, \mathrm{N}$, or $\mathrm{Zr})$ were considered in the calculation. The similar METALS program was used in reference [26] and postulation of adding various amounts of $\mathrm{C}$ and $\mathrm{Si}$ was made. Liquid with higher-than-normal amount of $\mathrm{Si}$ in alloy 718 seemed to become lighter with solidification. However, it is unlikely to occur in model alloys (HV8851 to HV8853) since the $\mathrm{C}$ and $\mathrm{Si}$ contents were intentionally reduced to minimize their effects on density.

\subsection{Summary}

The model alloys and experimental methods applied in the current study are summarized.

1. The following model alloy systems are used in the current study:

a. Ternary (Ni-Cr-Ti, Ni-Cr-Nb) and quaternary (Ni-Cr-Fe-Nb) alloys

b. $\mathrm{Ni}-20 \mathrm{Cr}-18 \mathrm{Fe}-\mathrm{Nb}-\mathrm{Ti}$

c. $\mathrm{Ni}-20 \mathrm{Cr}-36 \mathrm{Fe}-\mathrm{Nb}-\mathrm{Ti}$

d. Ni-20Cr-18Fe-Nb-Ti-Al

e. Industrial alloys 718,706 , and 625

2. Horizontal solidification setup is used to physically simulate freckle formation in model alloys HV8851 (RN904), HV8852 (RN903), and HV8853 (RN902).

3. Differential Thermal Analysis (DTA) is used to determine the characteristic temperatures of the alloys and fraction of solid versus temperature relations.

4. Optical microscopy, SEM, and EDS are used to analyze the microstructures and chemical compositions.

5. Thermodynamic calculation code, Thermo-Calc ${ }^{\mathrm{TM}}$, and NiFe-Database (version 3), are used for theoretical simulation of solidification in Ni-based alloys.

6. METALS, a program provided by NPL, UK, is used for liquid density calculation. The error of calculation is estimated to be less than $3 \%$ of experimental values. 
Table 2-1: Chemical compositions of model superalloys (wt pet, $\mathrm{Ti} / \mathrm{Nb}$ in at pet)

\begin{tabular}{|c|c|c|c|c|c|c|c|c|c|c|}
\hline Type & Heat & $\mathbf{N i}$ & $\mathrm{Cr}$ & $\mathrm{Fe}$ & $\mathbf{N b}$ & $\mathbf{T i}$ & Al & C & $\mathbf{S i}$ & $\mathbf{T i} / \mathbf{N b}$ \\
\hline \multirow{3}{*}{$\mathrm{Ni}-\mathrm{Cr}-\mathrm{Ti}$} & Design $^{\mathrm{a}}$ & 80 & 20 & & & 6 & & & & \\
\hline & HV8851 & 73.9 & 19.89 & & & 5.94 & & 0.0039 & 0.023 & \\
\hline & RN904 & 74.15 & 20.17 & & & 5.7 & & 0.009 & $<0.01$ & \\
\hline \multirow{3}{*}{$\begin{array}{c}\mathrm{Ni}-\mathrm{Cr}- \\
\mathrm{Nb}\end{array}$} & Design $^{b}$ & 71.4 & 20 & & 8.6 & & & & & \\
\hline & HV8852 & 71.06 & 20.13 & & 8.36 & & & 0.0067 & 0.036 & \\
\hline & RN903 & 70.57 & 20.09 & & 8.57 & & & 0.004 & $<0.01$ & \\
\hline \multirow{7}{*}{$\begin{array}{l}\mathrm{Ni}-\mathrm{Cr}- \\
\mathrm{Fe}-\mathrm{Nb}\end{array}$} & Design $^{c}$ & 55 & 20 & 18 & 7 & & & & & \\
\hline & HV8853 & 54.83 & 20.18 & 17.73 & 6.94 & & & 0.013 & 0.044 & \\
\hline & RN902 & 54.43 & 19.95 & 17.76 & 7.13 & & & 0.014 & 0.03 & \\
\hline & CK-30 & 47 & 20 & 18 & 15 & & & & & \\
\hline & CL-2 & 55 & 2( & 10 & 15 & & & & & \\
\hline & CL-3 & 55 & 12 & 18 & 15 & & & & & \\
\hline & HV8582 & 59.83 & 20.16 & 5.16 & 14.03 & & & 0.03 & 0.07 & \\
\hline \multirow{3}{*}{718} & \begin{tabular}{|l|} 
HV8549 \\
\end{tabular} & 50.59 & 19.93 & 18.25 & 5.33 & 5.55 & & 0.36 & 0.1 & 2.02 \\
\hline & HV8520 & 49.53 & 19.36 & 18.06 & 7.32 & 5.09 & & 0.11 & 0.12 & 1.35 \\
\hline & CL-4 & 50 & 20 & 18 & 8 & 4 & & & & 1 \\
\hline \multirow{4}{*}{706} & \begin{tabular}{|l|} 
HV8524 \\
\end{tabular} & 32.69 & 20.02 & 36.04 & 5.39 & 5.43 & & 0.05 & 0.11 & 1.95 \\
\hline & HV8523 & 32.81 & 20.32 & 36.47 & 5.75 & 4.27 & & 0.04 & 0.1 & 1.44 \\
\hline & HV8552 & 32.36 & 19.81 & 35.02 & 7.79 & 3.9 & & 0.04 & 0.12 & 0.97 \\
\hline & HV8586 & 41.81 & 16.06 & 35.37 & 3.23 & 2.94 & & 0.03 & 0.01 & 1.77 \\
\hline \multirow{4}{*}{$718+\mathrm{Al}$} & HV8550 & 48.55 & 19.94 & 18.09 & 7.72 & 4.01 & 1.12 & 0.04 & 0.09 & 1.01 \\
\hline & HV8522 & 49.19 & 19.19 & 17.38 & 7.41 & 5.32 & 1.13 & 0.04 & 0.11 & 1.39 \\
\hline & HV8521 & 49.18 & 20.73 & 18.83 & 5.62 & 4.24 & 1.18 & 0.05 & 0.1 & 1.46 \\
\hline & HV8551 & 49.56 & 19.69 & 18.21 & 5.37 & 5.59 & 1.14 & 0.04 & 0.09 & 2.02 \\
\hline \multirow[t]{2}{*}{$718+X$} & \begin{tabular}{|l|} 
HV8583 \\
\end{tabular} & 54.79 & 19.95 & 5.15 & 14.27 & & $5.01 \mathrm{Mc}$ & 0.15 & 0.09 & \\
\hline & HV8584 & 57.77 & 20.07 & 5.15 & 14.28 & & $1.95 \mathrm{Si}$ & 0.04 & 1.95 & \\
\hline
\end{tabular}

Note:

a, b, c: Ni-Cr-Ti alloy (HV8851, RN904), Ni-Cr-Nb (HV8852, RN903), Ni-Cr-Fe-Nb (HV8853, RN902).

1. 718 type $=\mathrm{Ni}-20 \mathrm{Cr}-18 \mathrm{Fe}-\mathrm{Nb}-\mathrm{Ti}$.

2. 706 type $=\mathrm{Ni}-20 \mathrm{Cr}-36 \mathrm{Fe}-\mathrm{Nb}-\mathrm{Ti}$.

3. $718+\mathrm{Al}=\mathrm{Ni}-20 \mathrm{Cr}-18 \mathrm{Fe}-\mathrm{Nb}-\mathrm{Ti}-1 \mathrm{Al}$.

4. $718+\mathrm{X}, \mathrm{X}=\mathrm{Mo}$ or $\mathrm{Si}$.

5. RN902 through RN904 are alloys melted at Daido Steel Corporation, Japan.

6. HV ingots are alloys melted at TPC, Special Metals Corporation, WV.

7. $\mathrm{CK}$ and $\mathrm{CL}$ alloys are from reference [23].

8. $\mathrm{Ti} / \mathrm{Nb}$ ratio is atomic ratio.

Table 2-2: Chemical compositions of industrial alloys 718, 706 and 625 (wt pct)

\begin{tabular}{cccccccccc}
\hline Alloys & $\mathbf{N i}$ & $\mathbf{C r}$ & $\mathbf{F e}$ & $\mathbf{M o}$ & $\mathbf{N b}$ & $\mathbf{T i}$ & $\mathbf{A l}$ & $\mathbf{C}$ & $\mathbf{S i}$ \\
\hline 718 & 54.01 & 18.19 & 17.77 & 3.01 & 5.41 & 0.98 & 0.56 & 0.04 & 0.03 \\
706 & 41.39 & 16.3 & 37.03 & 0.12 & 2.98 & 1.7 & 0.19 & 0.01 & - \\
625 & 60.28 & 22.09 & 4.4 & 9.1 & 3.53 & 0.29 & 0.26 & 0.02 & 0.03 \\
\hline
\end{tabular}




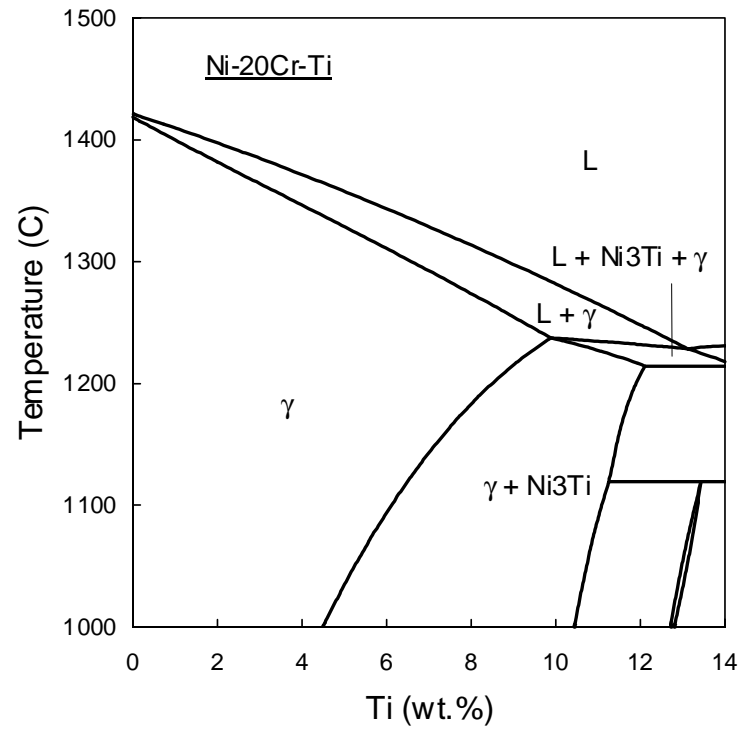

(a)

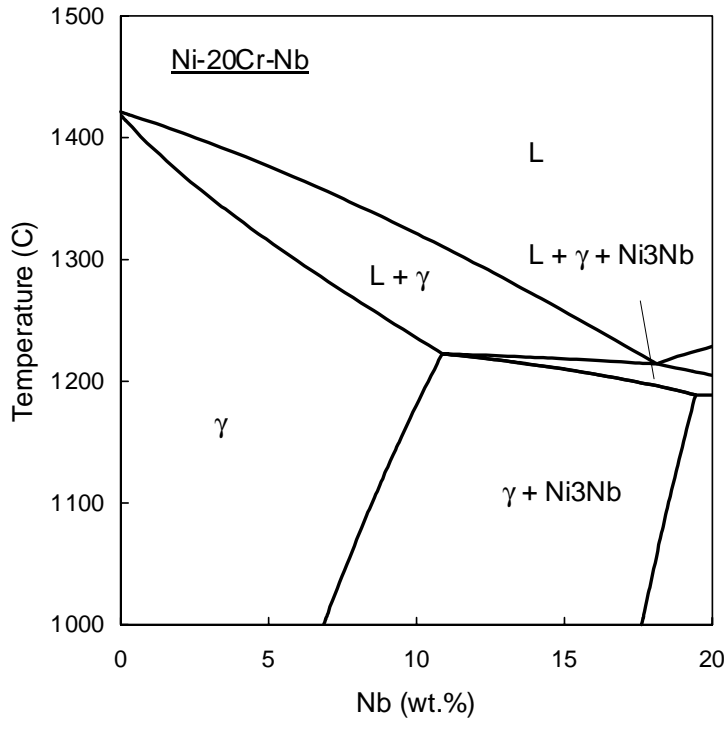

(b)

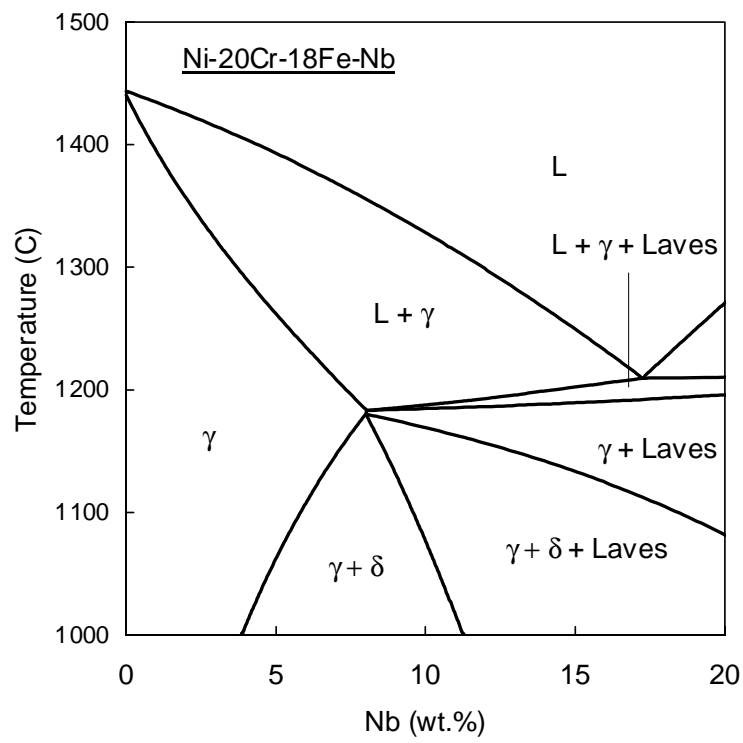

(c)

Figure 2-1: Isopleth of (a) Ni-20Cr-Ti, (b) Ni-20Cr-Nb, and (c) Ni-20Cr-18Fe-Nb, calculated by Thermo-Calc. 


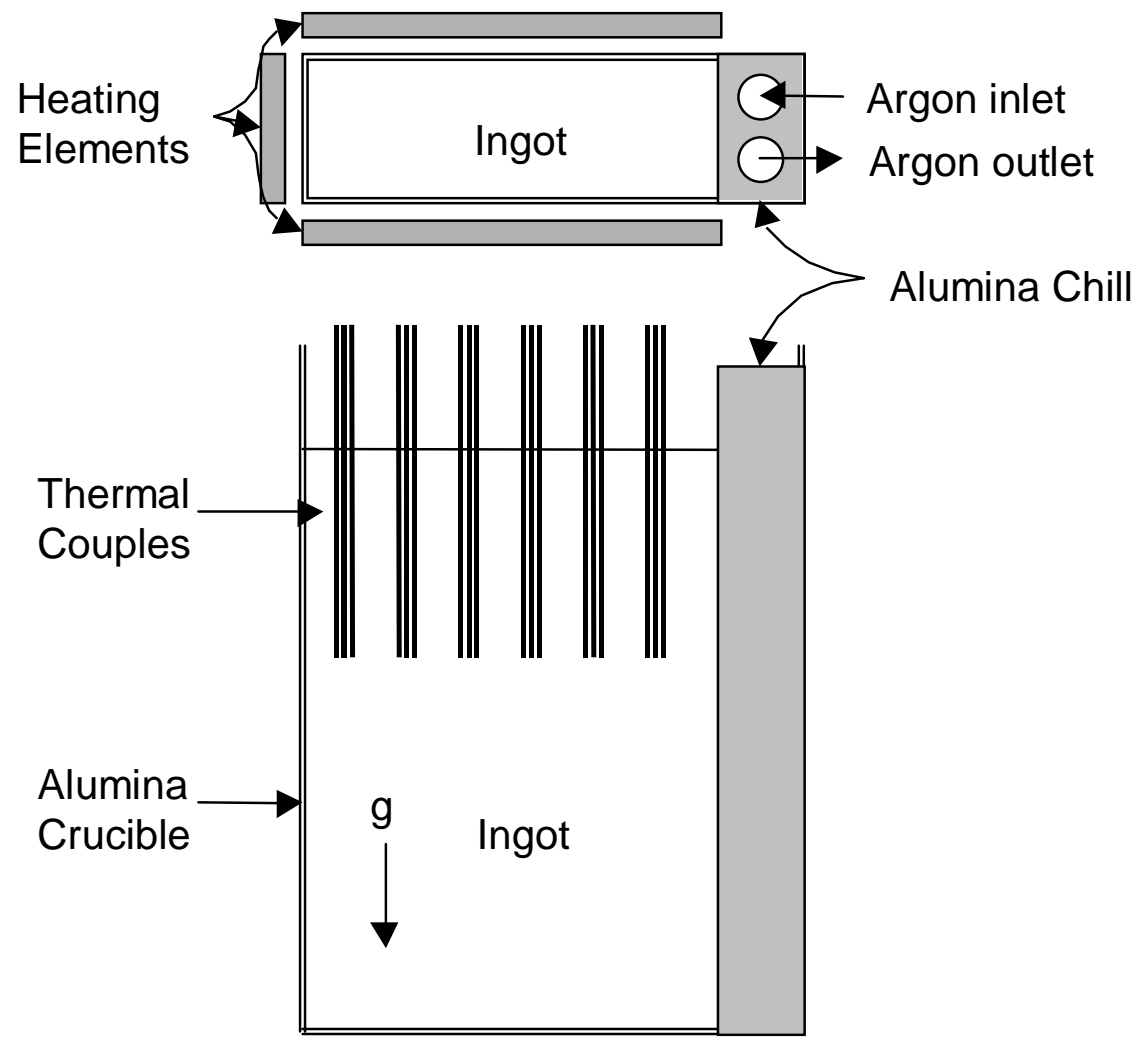

Figure 2-2: Schematic of horizontal solidification setup (top view and side view). 
Chapter 3

\section{Freckling Mechanism in Ni-based Superalloys}

3.1. Introduction 32

3.2. Freckling in an Alloy 625 Ingot 32

3.2.1. Freckle Appearance 33

3.2.2. Discussion 34

3.3. Physical Simulation of Freckling in Model Superalloys 34

3.3.1 Freckle Appearance 35

3.3.2 Channel Initiation 37

$\begin{array}{lll}\text { 3.3.3 Temperature Data } & 38\end{array}$

3.3.4 Secondary Dendrite Arm Spacing $\quad 39$

3.4. Mechanism of Freckle Formation 40

3.4.1. Isotherms 41

3.4.2. Solidification Front (Freezing Front) 42

3.4.3. Iso-fraction Solid 43

3.4.4. Freckling Mechanism 43

3.4.5. Application to Remelting Process 45

3.5. Key Controlling Parameters 46

$\begin{array}{lll}\text { 3.6. Summary } & 47\end{array}$ 


\subsection{Introduction}

The puzzle of freckling phenomenon in superalloys is associated with heavy alloy additions such as $\mathrm{Nb}$ and Mo. These elements are very sensitive to dendritic segregation, and freckles are likely to occur in a superalloy containing a certain amount of $\mathrm{Nb}$. The density inversion theory, which describes the buoyancy force for interdendritic liquid flow, was accepted in general to explain freckling in superalloys containing $\mathrm{Al}$ and Ti. However, it faces difficulty in Nb-containing superalloys. The aim of present study is to develop a physical model for freckle formation in superalloys. It is critical to identify the key control parameters for freckling that are measurable attributes during solidification.

This chapter begins with the examination of freckles in an alloy 625 billet, which contains $\mathrm{Nb}$ and $\mathrm{Mo}$ as the major strengthening elements. Three model alloys were designed by purposely adding $\mathrm{Nb}$ or $\mathrm{Ti}$ in the alloys to generate density gradient in the interdendritic liquid during solidification. Favorable processing conditions for freckle formation are physically simulated in horizontal solidification setup. Key control parameters for freckling are identified through investigation of alloying chemistry, structural features and temperature data of freckled alloys.

\subsection{Freckling in an Alloy 625 Ingot}

Alloy 625 contains $\mathrm{Nb}$ and Mo as strengthening elements and is a typical case of having heavy solute elements in the interdendritic region. Freckles in a vacuum arc remelt (VAR) alloy 625 billet has been analyzed in this section. There was a recorded power interruption during the VAR process. The ingot is 33 inch in diameter and was forged to 16 inch square billet before the freckled area was cut out. The nominal and actual chemical compositions are given in Table 3-1.

Table 3-1: Nominal and Actual Chemical Compositions of Alloy 625 (wt pct)

\begin{tabular}{cccccccccc}
\hline & $\mathrm{Ni}$ & $\mathrm{Cr}$ & $\mathrm{Fe}$ & $\mathrm{Mo}$ & $\mathrm{Nb}$ & $\mathrm{Ti}$ & $\mathrm{Al}$ & $\mathrm{C}$ & $\mathrm{Si}$ \\
\hline Nominal & Balance & 21.5 & 2.5 & 9.0 & 3.6 & 0.2 & 0.2 & 0.05 & 0.2 \\
Actual & Balance & 22.09 & 4.40 & 9.10 & 3.53 & 0.29 & 0.26 & 0.024 & 0.03 \\
\hline
\end{tabular}




\subsubsection{Freckle Appearance}

The macrostructure of longitudinal sections of the 625 billet illustrates the face and side view of freckles, as shown in Figure 3-1. The freckle spots are stretched into elliptical shapes in longitudinal direction because of the forging deformation and the cut-up surface location. The distribution of spots clearly outlines the molten pool profile in the VAR process. The average width of the freckles is in the order of millimeters. Numerous tiny spots that have sizes less than $1 \mathrm{~mm}$ are visually observed in the vicinity of the big spots. The length of the trail shown in the side view of Figure 3-1 (b) is about $100 \mathrm{~mm}$.

Figure 3-2 shows the enlarged views of both the transverse and longitudinal cross sections of freckles. It has sharp, well-defined boundary at bottom side, which is close to the bottom of the molten pool, and diffuse boundary at topside. These features are believed to imitate the freckles in as-cast condition since the forging process has little effect on changing the macroscopic segregation. It only changes the curvature of the pool shape through the forging deformation.

Scanning electron microscopy (SEM) illustrates that freckles are actually mixture of network with excessive amounts of heavy solute elements (bright contrast in BSE mode) and islands of normal composition, as shown in Figure 3-3. Segregation are more concentrated in the bottom, where the boundary is sharp and well-defined, and less concentrated as close to the top until the boundary becomes blurred and indistinguishable with the matrix. The lower portion of the freckled area contains significant amount of large globular Laves phase, as seen in Figure 3-4. In areas approaching the upper region, the size and the amount of Laves are both reduced. Arrays of fine needle-shaped precipitated decorate the grains between the globular phases. These phases are identified to be $\delta-\mathrm{Ni}_{3} \mathrm{Nb}$.

An elemental scan using EDS across the freckled area is shown in Figure 3-5. It can be seen that the freckled area is enriched in $\mathrm{Nb}$ and $\mathrm{Mo}$, lean in $\mathrm{Ni}$ and $\mathrm{Cr}$, with $\mathrm{Fe}$ content slightly lower than the surrounding matrix. Quantitative chemical analysis of the precipitates by EDS is given in Table 3-2. Laves phase is significantly enriched with $\mathrm{Nb}$ and Mo. $\delta-\mathrm{Ni}_{3} \mathrm{Nb}$ is enriched in $\mathrm{Nb}$. Both of these phases are $\mathrm{Nb}$-rich products, which indicates that freckles are rich in heavy solute elements. 
Table 3-2: Chemical Composition of Phases in Freckles in Alloy 625 (at pct)

\begin{tabular}{lccccccc}
\hline & $\mathrm{Ni}$ & $\mathrm{Cr}$ & $\mathrm{Fe}$ & $\mathrm{Mo}$ & $\mathrm{Nb}$ & $\mathrm{Ti}$ & $\mathrm{Al}$ \\
\hline$\delta$ - $\mathrm{Ni}_{3} \mathrm{Nb}$ & 67.28 & 5.19 & 1.11 & 4.74 & 19.98 & 0.84 & 0.85 \\
Laves & 30.11 & 18.2 & 2.06 & 27.77 & 21.25 & 0.30 & 0.30 \\
\hline
\end{tabular}

\subsubsection{Discussion}

The addition of $\mathrm{Nb}$ in Ni-based superalloys promotes the precipitation of coherent $\gamma^{\prime \prime}$ strengthening phase, while it also causes the formation of Laves phase during solidification. $\mathrm{Nb}$ segregation is the controlling factor in the development of solidification microstructure in these alloys. The existence of excessive amounts of Laves and $\delta$ phase in the freckled region indicates that the channel segregate is a collection of the interdendritic liquids that are rich in normally segregating $\mathrm{Nb}$ (partition ratio less than 1). These phases also contain high levels of Mo, whose atomic weight is higher than $\mathrm{Ni}$. The $\mathrm{Al}$ and $\mathrm{Ti}$ contents are limited in the alloy and $\mathrm{C}$ and $\mathrm{Si}$ levels are considered to be negligible. Hence, the density of liquid in mushy zone is evidently dominated by the segregation of heavier elements, $\mathrm{Nb}$ and Mo.

The gradual increase of the size and amount of Laves from the topside to the bottom side of freckles implicates that heavy interdendritic liquids seep through dendrite array and agglomerate into solute-enriched region, eventually solidify into freckles.

The sharp boundary at bottom side and diffuse boundary at topside of freckle channels is a unique feature in remelting process that has been more or less omitted in other studies. A similar freckle morphology was shown in as-cast ESR alloy 718, however, no explanation was given [8]. This unique appearance, together with visual, chemical, and metallographic data, specify a set of observations that a successful model for freckling in remelting process must explain. A detailed freckling mechanism will be described in the following sections through the physical simulation of freckle formation.

\subsection{Physical Simulation of Freckling in Model Superalloys}

Freckles in production ingots are often found by "accident" after ingot conversion to billet and no detailed thermal history and as-cast structure can be obtained. Hence, it is preferred 
that freckles are generated under controlled solidification conditions with specially designed alloy chemistry. The physical simulation technique can provide significant insights for the understanding of freckling problem in real production.

Physical simulation of freckle formation is realized using the horizontal solidification setup described in chapter 2. Three model alloys, Ni-20Cr-6Ti, Ni-20Cr$8.6 \mathrm{Nb}$, and $\mathrm{Ni}-20 \mathrm{Cr}-18 \mathrm{Fe}-7 \mathrm{Nb}$ (see Table 2-1 for compositions) were used for horizontal solidification. Freckles were observed in all three alloys. The ingots were sectioned in the longitudinal planes and macro-etched for visual examination. One of the ingot halves was used for microscopic investigation. Temperatures were recorded versus time during each of the runs and are presented in the following sections.

\subsubsection{Freckle Appearance}

\subsubsection{Visual Examination}

\section{Ni-20Cr-6Ti (HV8851-Special Metals Corp., RN904-Daido Steel Corp.)}

Columnar grains in alloy HV8851 are shown in Figure 3-6(a), and these grains are tilted at an angle of 50 degrees from the horizontal plane. Three channels can be clearly seen visually on the longitudinal plane and they seem to be associated with segregation in the lower portion of the ingot. It is seen that two small channels emerge from the region and gradually grow into one as the solidification proceeds away from the chill. A lot of freckle channels are seen on the transverse cross section of the ingot, however, no specific number can be obtained due to the poor contrast between the freckles and the matrix. The angle between the longitudinal axis of the columnar grains and the horizontal plane is about 50 degree. The angle is defined as the solidification front angle in this study.

\section{$N i-20 C r-8.6 N b(H V 8852, R N 903)$}

Only one freckle channel is seen on the upper half of the longitudinal section of HV8852, as shown in Figure 3-6(b). The freckle appears like black streak probably due to the excessive eutectic precipitation in the freckled region. Two freckles are observed on one half of the transverse cross section. The columnar grains are tilted at an angle of 40 degree with respect to the horizontal plane. 
$\mathrm{Ni}-20 \mathrm{Cr}-18 \mathrm{Fe}-7 \mathrm{Nb}(\mathrm{HV} 8853, \mathrm{RN} 902)$

At least four freckle channels are seen on the longitudinal section of HV8853, two of which stretch from the top continuously to the bottom of the ingot (Figure 3-6(c)). These channels are aligned in the vertical direction with their tops being inclined towards the chill surface. 15 channels are seen on one half of the transverse cross section of the ingot. The columnar grains are tilted at an angle of 30 degree.

\subsubsection{Microstructures}

Metallographic specimens were cut from locations on the ingots as shown in Figure 3-7 (a) to (c). These specimens were prepared using the procedure described in chapter 2. Following analysis were performed: (1) Microstructure of freckles; (2) Measurement of secondary dendrite arm spacing, $d_{2}$, with respect to $x$, the distance from the chill surface.

\section{Ni-20Cr-6Ti}

The transverse sections of freckles in HV8851 are shown in Figure 3-8 (a) and (b), with directions of crystal growth and gravity force given on the photomicrographs. It is seen that freckles appear as dark spots on the transverse surface and are intermixed with long needle-shaped phases. The needle phase can be as long as the width of the freckle spot and has higher incidence near freckles. They make it difficult to visually differentiate the boundary between freckles and matrix.

One of the transverse cross sections is enlarged using SEM with the top and bottom regions shown in Figure 3-8 (c) and (d) respectively. The needle-shaped phase is identified to be $\eta-\mathrm{Ni}_{3} \mathrm{Ti}$, which are precipitation products in solid state, while the eutectic $\eta$ is formed at the end of solidification.

\section{$\mathrm{Ni}-20 \mathrm{Cr}-8.6 \mathrm{Nb}$}

Transverse sections of two freckles are illustrated in Figure 3-9 (a). They are more clearly distinguished from the surrounding matrix than HV8851, with sharp boundaries at solidus side (close to solidus) and diffuse boundaries at liquidus sides (close to solidification front). This feature is also evident in the longitudinal section of freckles, as shown in 
Figure 3-9 (b). Two types of phases are identified in the freckled region, the eutectic $\delta$ $\mathrm{Ni}_{3} \mathrm{Nb}$ (bright phase in Figure 3-9 (d)) and needle-shaped $\delta-\mathrm{Ni}_{3} \mathrm{Nb}$. More eutectic $\delta-\mathrm{Ni}{ }_{3} \mathrm{Nb}$ phases are observed on the solidus side of freckles, which indicates more $\mathrm{Nb}$-segregation.

\section{$\mathrm{Ni}-20 \mathrm{Cr}-18 \mathrm{Fe}-7 \mathrm{Nb}$}

The transverse cross section of freckle is shown in Figure 3-10 (a), with sharp boundary on the solidus side and diffuse boundary on the liquidus side. It is clear that dendrite arms are completely melted at the solidus side of freckle defining a sharp boundary, while remnants of incompletely melted dendrite debris are mixed with freckle liquid that blurs the boundary on the liquidus side. It is also very clear in the longitudinal section of freckles (Figure 3-10 (b)). The dendrites are melted regardless of their orientation at the solidus side. It appears that the freckle line lies at an angle with the secondary dendrite arms formed in front of the freckle.

The eutectic phases inside the freckled area are shown in Figure 3-10 (d) and they are mixtures of Laves, $\delta-\mathrm{Ni}_{3} \mathrm{Nb}$ and $\gamma$ phase. Small amount of $\mathrm{NbC}$ was also observed in freckles. The intimate feature implicates that they formed at similar temperatures.

\subsubsection{Channel Initiation}

Extensive research has been done on freckles, however, few results have been published on how these channel segregates initiate during solidification process. It is proposed that freckles originate from widening interdendritic channels in the interior of vertically directionally solidified $\mathrm{Pb}-\mathrm{Sn}$ ingot in which freckling is controlled by density inversion [18]. Bridge and Beech [44] implied that major segregates originate at irregularities on the growth front resulting in the entrapment of a significant pocket of liquid by the advancing front. The large driving force for fluid flow would accentuate any instability caused by the irregular interface. However, no evidence of freckle initiation in Ni-based superalloys has been given.

Considering the $\mathrm{Ni}-20 \mathrm{Cr}-6 \mathrm{Ti}$ alloy, at the start of freezing the $\mathrm{Ti}$ rich liquid between the dendrites is lighter than the bulk liquid and tends to move upwards. Vertical motion is only possible if liquid moves away from the chill at the bottom of the solidifying ingot. One of such evidence is shown in Figure 3-11. Two fine channels are seen to flow 
upwardly away from the chill and then converged as the two crossed over. One channel was developed from the space between the primary dendrites and the other one is associated with irregular dendrites. The widths of the channels are in the same order as the primary arm spacing and are both enriched with eutectic $\eta$ phase. Some micro-porosity is found in one channel, indicating the liquid solidified at last. The observation indicates that freckles originate from irregularities at the solidification front. Due to the tilted angle of the primary dendrites, space between the arms provided the preferred flow channel.

It is anticipated that freckles would originate from the $\mathrm{Nb}$-enriched heavier liquid in $\mathrm{Ni}-\mathrm{Cr}-\mathrm{Nb}$ and $\mathrm{Ni}-\mathrm{Cr}-\mathrm{Fe}-\mathrm{Nb}$ alloys following a similar pattern from top of the ingots. Unfortunately, no sample was available to trace the channels back to their origin. However, a large number of such irregularities would be expected in any dendritic growth situation, as illustrated clearly in the freckle channel which is located near the bottom of HV8853 ingot (Figure 3-12). The interdendritic liquid flow out of the opening between disordered primary dendrites, then continue to flow down through possible opening spaces while the direction is averted more vertical as it becomes bigger in width. The interdendritic liquid seems to feed the main stream from above.

These observations provide solid evidence that freckles in Ni-Cr-Ti alloy is caused by the flow of lighter interdendritic liquid enriched with $\mathrm{Ti}$, while the freckles in $\mathrm{Nb}$ containing alloys are caused by downward flow of heavier interdendritic liquid.

\subsubsection{Temperature Data}

Temperatures during solidification were recorded by inserting six thermocouples (T/C) along the growth direction. Figure 3-13 shows the temperature distributions along the distance from chill at various time intervals. Liquidus temperature is defined as the peak value on the $T(t)$ curve before the temperature begins to drop. The liquidus temperatures are $1374^{\circ} \mathrm{C}$ for $\mathrm{RN} 904,1364^{\circ} \mathrm{C}$ for $\mathrm{RN} 903$, and $1351^{\circ} \mathrm{C}$ for RN902. Thus the distance (x) of the freezing front from chill, and the distance of isotherms at $50^{\circ} \mathrm{C}$ or $20^{\circ} \mathrm{C}$ below liquidus, are shown in Figure 3-14 (versus time). The cooling rate at the location of T/C is obtained to be the time for the liquid to cool from the liquidus to $50^{\circ} \mathrm{C}$ below (Figure 3-15 (a)). On the other hand, the temperature gradient, $G$, which was defined as the mean temperature gradient in the region from freezing front to the position $50^{\circ} \mathrm{C}$ below liquidus, 
is shown in Figure 3-15 (b). Because the solidification proceeds away from the side chill, the movements of growth front and the solidus position become increasingly apart with time. The local growth rates of liquidus and the isotherms that are $50^{\circ} \mathrm{C}$ (or $20^{\circ} \mathrm{C}$ ) below liquidus were taken as the slopes of those shown in Figure 3-14. These terms have the same unit as $R$, the solidification rate, and they are shown in Figure 3-15 (c).

In this study, all of the ingots were solidified under controlled conditions in attempt to prolong the local solidification time that would provide favorable conditions for freckling. Solidification ranges (difference between liquidus and solidus from DTA experiments) were used as references for cooling control by adjusting the air flow rate through the cooling block. It can be seen that the cooling rates, temperature gradients, and local growth rates continued to decrease as the solidification fronts moved away from chill. As a result, the local mushy zone depths, which are defined as the distance between liquidus and $50^{\circ} \mathrm{C}$ ( or $20^{\circ} \mathrm{C}$ ) below, increase with time and can be as large as $40 \mathrm{~mm}$. This value is in the same order of magnitude as those observed in production VAR process. For example, the pool depth changes from $148 \mathrm{~mm}$ at center of the ingot to $86 \mathrm{~mm}$ at the edge for $508 \mathrm{~mm}$ in diameter VAR ingot at a melt rate of $276 \mathrm{~kg} / \mathrm{hr}$ [8].

\subsubsection{Secondary Dendrite Arm Spacing (SDAS)}

Secondary dendrite arm spacings of HV8852 and HV8853 were measured on the transverse cross sections of the samples shown in Figure 3-7. The measurements were carried out on each sample by dividing the distance of several equally spaced secondary arms with the number of spacings included. These values were then averaged and the standard deviation of measurements was calculated. The results are shown in Table 3-3 and Figure 3-16.

It is noted that the secondary arm spacings for the two alloys are remarkably different although their cooling rates are similar. Correlation between cooling rate $(\dot{\varepsilon}$, $\left.{ }^{\circ} \mathrm{C} / \mathrm{sec}\right)$ and $d_{2}(\mu \mathrm{m})$ is calculated for both alloys. For HV8852, $d_{2}=134.93 \cdot \dot{\varepsilon}^{-0.0979}$,

adjusted R-square $=0.98951$. For HV8853, $d_{2}=111.54 \cdot \dot{\varepsilon}^{-0.1838}$, adjusted R-square $=$ 0.90914. The last two data points of $d_{2}$ in each alloy were rejected because solidification at these locations started from the opposite side of chill surface. Since there is no difference in cooling rate, the variation of $d_{2}$ can be attributed to the composition effect. Both alloys 
have $\mathrm{Nb}$ additions, while HV8853 contains 18 wt pct of Fe. Future study is needed to substantiate the composition effect on $d_{2}$.

\begin{tabular}{c|cc|cr}
\multicolumn{4}{c}{ Table 3-3: SDAS of horizontal solidification ingots $(\boldsymbol{\mu m})$} \\
\hline$x(\mathrm{~mm})$ & \multicolumn{2}{|c|}{ HV8852 } & \multicolumn{2}{c}{ HV8853 } \\
\hline 8.33 & 145.61 & $(16.23)$ & 106.28 & $(11.72)$ \\
25.00 & 173.91 & $(33.83)$ & 123.12 & $(10.03)$ \\
41.67 & 178.64 & $(19.14)$ & 163.88 & $(12.28)$ \\
58.34 & 179.68 & $(12.35)$ & 201.99 & $(16.27)$ \\
75.01 & 193.28 & $(67.42)$ & 248.13 & $(18.97)$ \\
91.68 & 116.35 & $(13.79)$ & 236.01 & $(16.46)$ \\
108.35 & 107.48 & $(19.54)$ & 208.01 & $(34.83)$
\end{tabular}

" $\mathrm{x}$ " is the distance from chill. Numbers in parenthesis are the standard deviations of measurements.

The permeability, which is a measure of the fluidity resistance of the medium, is anisotropic because of the directional nature of the columnar-dendritic structure. In horizontal solidification, interdendritic liquid flows in the direction that is normal to the primary dendrite arms where the permeability depends upon the secondary dendrite arm spacing $\left(d_{2}\right)$ as well as the primary arm spacing $\left(d_{1}\right)$ and liquid fraction $\left(f_{\mathrm{L}}\right)$. This direction is parallel to the direction of gravity force. In alloy HV8852, $d_{2}$ slightly increases from $146 \mu \mathrm{m}$ at the beginning of solidification to $193 \mu \mathrm{m}$ at $80 \mathrm{~mm}$ from chill. In HV8853, it increases remarkably from $106 \mu \mathrm{m}$ at the beginning of solidification to $248 \mu \mathrm{m}$ at $80 \mathrm{~mm}$ from chill. The drastic increase of dendrite arm spacing in HV8853 caused the permeability to increase with the progress of solidification, hence, the freckle flows would become increasingly severe. It is analogous to the melt rate transients observed in remelting which induces significant increase of dendrite arm spacings. This is consistent to the number of freckles observed in both of the alloys.

\subsection{Mechanism of Freckle Formation}

The mechanism of channel flow depends on the physical structure at the interface between bulk liquid and the solidifying solid, namely the mushy zone. Several structural features can be defined in mushy zone, such as the solidification front, isotherms, iso-fraction solid, 
and mushy zone depth, according to the local temperature profile or the fraction ratio between liquid and solid. These structural characteristics, together with the density difference inside the mushy zone, contribute to the formation of freckles in these alloys. In this section, they are described under the horizontal solidification condition. The purpose of the present study is to develop a freckling mechanism that can successfully explain the occurrence of freckles in the physical simulation of model alloys. Applicability of the mechanism to remelting process will be discussed. Key control parameters for freckling are identified.

\subsubsection{Isotherms}

Isotherm is a contour line on which every point has the same temperature. Direction normal to isotherms represents the direction of maximum thermal gradient, i.e. the heat flow direction. Columnar grains grow against the heat flow direction. In vertically directional solidification, heat is extracted from the bottom of ingot and the isotherms are parallel to solidification front so that the columnar grains are aligned in the vertical direction.

In the horizontal solidification of model alloys, tilted columnar grains are seen in all cases and isotherms can be drawn as a set of parallel lines perpendicular to the long axis of the grains. This is illustrated in Figure 3-18. Thus the overall temperature gradient across the ingot is tilted. This is attributed to positive temperature gradient between the top and the bottom of the solidifying ingot. After pouring, exothermic material was placed on top of the melt to prevent it from oxidation. This keeps the top surface hotter than the bottom surface of the ingot where there is no heat source. Heat sinks at the chill surface and the bottom surface would result in a tilted direction for heat flow, i.e. a tilted temperature gradient. The tilt angle is identical to the solidification front angle, which is defined as the angle between the long axis of the columnar grain (heat flow direction) and the horizontal plane. The angle can be adjusted by changing the gas flow rate through the chill block and the amount of exothermic on top of the ingot. 


\subsubsection{Solidification Front (Freezing Front)}

Solidification front is the interface between the bulk liquid and the mushy zone, where the fraction of liquid equals one and the temperature equals to the liquidus of the bulk liquid. Since the chemistry of the bulk liquid is different from location to location, the temperature of freezing front may also vary.

No direct observation of the movement of solidification front is possible in the present study. However, several small-scale castings under similar conditions have been quenched or recorded by X-ray camera in real time to obtain the ingot structure during the solidification process [44]. Figure 3-17 shows a sequence of photographs taken during solidification of $\mathrm{Al}$ with $20 \mathrm{wt}$ pct $\mathrm{Cu}$ alloy [44]. Channel segregates were observed and the solidification front was retarded at the bottom of the ingot. $\mathrm{Cu}$ is heavier than $\mathrm{Al}$, which results in positive density gradient along the mushy depth. Since Nb-containing model alloys have similar positive density gradients, curvatures of solidification fronts are expected.

Two effects may contribute to the curvature of solidification front. Here Nbcontaining alloys are used for illustration. First, it is the result of bulk liquid convection caused by the settling of solute atoms during solidification. Since $\mathrm{Nb}$ is heavier than $\mathrm{Ni}$, the $\mathrm{Nb}$ atoms will tend to settle to the bottom of ingot which results in the enrichment of $\mathrm{Nb}$ in that region [13]. Second, the continuous flow of channels pushes the $\mathrm{Nb}$-rich liquid into the bulk liquid at bottom of ingot. Based on phase diagram principle, the Nb-rich liquid at the bottom would have a lower liquidus temperature than that of the liquid at the top. The overall temperature gradient across the solidifying ingot should be tilted and uniform as it is determined by the furnace setup. Thus the freezing front of $\mathrm{Nb}$-containing alloy should be more vertical than the isotherms, which would otherwise be parallel to the isotherms. The situation in the Ni-Cr-Ti alloy is reversed. In this case Ti atoms tend to move upwards so that Ti enriched liquid would stay at top of ingot and the freezing front lags in this region. This would result in a solidification front that is parallel to the isotherms. The postulated curvatures of freezing front in model allots are illustrated in Figure 3-18. Direct proof of the curvature effect was not possible. However, it is reasonable to assume that the convection would occur in the bulk liquid during solidification permitted by the prolonged solidification time. Macrosegregation of solute 
elements along the vertical axis of the ingot would provide indirect evidence for such bulk liquid convection.

\subsubsection{Iso-Fraction Liquid}

During dendritic solidification, fraction of solid, i.e. the ratio between the volume (or weight) of solid and the total volume of metal in the mushy region, gradually changes from 0 at the top to 1 at the bottom of mushy zone. Iso-fraction solid refers to the contour line on which each point has the same fraction of solid. For vertical DS, iso-fraction solids are identical to the isotherms since the bulk liquid is of uniform composition.

In horizontal solidification, the situation is complicated by the non-uniform solute concentrations in the bulk liquid. As shown by the schematic phase diagram in Figure 319 , bulk liquid of lower concentration (alloy $1, \mathrm{C}_{\mathrm{L} 1}$ ) with a temperature drop of $\Delta \mathrm{T}$ from its liquidus $\left(\mathrm{T}_{01}\right)$ will have a fraction solid of bc/ac. While bulk liquid of higher concentration (alloy 2, $\mathrm{C}_{\mathrm{L} 2}$ ) will have a fraction solid of ef/df with the same $\Delta \mathrm{T}$. Obviously, bc/ac is greater than ef/df. Thus, alloy 1 has greater fraction solid than alloy 2 with same temperature drop from liquidus. In other words, in order to have the same fraction of solid, alloy 2 will need to have greater decrease in temperature. Contours of iso-fraction solids in both $\mathrm{Ti}$ and $\mathrm{Nb}$ containing alloys are illustrated in Figure 3-18. In Nb-containing alloys in which the solidification surface is retarded at the bottom of ingot, the iso-fraction solid lies at angle with respect to the isotherms and inclines more vertically. In Ti-containing alloy, the iso-fraction solid contours lied more horizontally than the isotherms.

\subsubsection{Freckling Mechanism}

During horizontal solidification of model alloys, solute elements redistribute into either liquid or solid, resulting in large density gradient of liquid inside mushy zone. Under the influence of gravity force, the interdendritic liquids tend to flow out of their interdendritic pockets and along preferred directions. The prevalence of irregularities at the freezing front enables these small streams of liquid to agglomerate into large streams, often through spaces between dendrite arms, as shown previously in Figures 3-11 and 3-12.

Once the channel is developed, it will move in the direction where the resistance of the surrounding medium is the smallest and the driving force of flow maintains maximum. 
The resistance term is represented by permeability, which is anisotropic in columnar structures, assuming other factors are constant. It is shown that flow perpendicular to the direction of the primary dendrites should be noticeably easier than that parallel to dendrites due to a permeability 2.5 to 4 times greater [26]. The driving force term is described by the density difference along the mushy depth. Based on phase diagram, liquid at higher temperature will have less solute content. Thus a pocket of liquid in a cool region will tend to move to a higher temperature region where the liquid is less dense (for both $\mathrm{Nb}$ and Ti alloys). Under the influence of gravity, flow will align itself to the vertical (gravity) direction, either upward or downward.

In Nb-containing model alloys, iso-fraction solid lies at an angle that is more vertical with respect to the isotherms. However it is reasonable that the angle is very small. Under the influence of gravity, the channel tries to align itself with a route that is vertical and has enough permeability for flow. From the macrostructure of the ingot, the channel moves in a direction that is parallel to the isotherm in the upper part of the ingot. That is through the space perpendicular to primary dendrites where the permeability is high. As it flows down more and becomes wider, the channel moves to a more vertical direction. It means that at early stage of development, freckle channel needs greater driving force and permeability in order to maintain a continuous momentum. As it becomes wider and contains enough mass in its stream, it prefers the shortest path with respect to the gravity direction.

For Ni-Cr-Ti alloy, iso-fraction solid lies more horizontally than isotherms. The channels move in a more vertical direction with respect to isotherms, which favor a greater driving force and smaller flow resistance. This results in nearly vertical freckle trails in HV8851 ingot.

For channel initiation, the driving force must exceed the resistance of the system. The severity of the channel segregation, i.e. the number of freckles, is determined by how much the driving force exceeds the resistance of the solidifying system, that is the ratio of the two terms. The Nb-containing model alloys form freckles by downward flow of interdendritic liquid. However, only 2 freckles were detected in HV8852, while 15 were found in HV8853. This must be related to the driving force as well as the resistance in the system, as explained by the Rayleigh number developed in chapter 6. 
In order to increase the volume of liquid in the stream, solute-rich interdendritic liquid from surroundings need to feed the channel as it flows and melts the dendrite arms on its path. The solute-rich liquid has a lower liquidus temperature than solute-lean dendrites, hence, it remelt the dendrites on both sides of the stream. The well-developed dendrites on the solidus side are melted and freeze again once the solidification passes the region. Therefore, a sharp boundary forms at the solidus side. However, the liquidus side has higher temperature and it takes longer for the region to melt and solidify so that dendrites can re-grow from the region. The remelt process also may not be complete due to higher temperature. Hence, diffuse boundary results. The appearance is in agreement with the observation of freckles in alloy 625. The feeding mostly comes from the interdendritic liquids on the liquidus side where the remelt process is not complete. Such evidence can be found in Figure 3-12.

\subsubsection{Application to Remelting Process}

The horizontal solidification assimilates the horizontal component in remelt process where freckles have the highest propensity to form. The main difference between the two processes is the curvature of the freezing front. For Nb-containing superalloys, the front is retarded at bottom of the ingot and becomes more vertical in horizontal solidification process. However, it changes gradually from vertical to horizontal orientation as it approaching the ingot center in remelting process. As a result, freckle channels become more horizontal as they flow toward the center of the ingot. It is illustrated in Figure 3-20.

However, the difference in the freezing front curvature will not affect the applicability of the horizontal solidification method. This can be explained by the fact that most of the freckles in remelt ingots are found in the mid-radius region where the slope of the freezing front is steep and vertical. As the front becomes horizontal toward the center, freckles are likely to be terminated. This is due to the diminishing effect of driving force for channel flow. Thus the horizontal solidification correctly simulates the horizontal component in remelting process where freckles are most likely to form for $\mathrm{Nb}$-containing superalloys. For superalloys with light solute elements such as $\mathrm{Ti}$ and $\mathrm{Al}$, horizontal solidification correctly represents the remelting process. In conclusion, the result obtained 
from the physical simulation in this study can be applied to assess the freckling potential for different Ni-based superalloys.

\subsection{Key Controlling Parameters}

Freckle potential and severity are the two concepts frequently used to describe the freckling behavior and sometimes no attempt is made to differentiate between the two. According to density inversion theory, freckle potential is determined only by the liquid density gradient. However, as shown in this study, freckle potential and severity are dependent on the driving force as well as the resistance of the system. In this way, both freckle potential and freckle severity are controlled by the key control parameters that will be shown below.

From the analysis in section 3.4, freckle channel flows upward when the liquid density inversion incurs, while it flows downward if heavy solute elements such as $\mathrm{Nb}$ partitions into liquid and causes negative density gradient. It can be rationalized that uniform distribution of density of the interdendritic liquid could be obtained by balanced additions of both light and heavy elements. Nevertheless, the way the liquid density changes in mushy zone provides the driving force for freckling. The resistance of liquid flow in the system also plays a vital role in that if the resistance is great enough the severity of flow can be reduced. The permeability is used to describe the easiness for

liquid flow and is determined by dendrite arm spacing, $d_{1}$ and $d_{2}$, and the fraction of liquid in mushy zone. Little knowledge is available for the composition effects on these factors.

Based on the analysis, the key control parameters for freckling in superalloys can be identified as follows:

1) Density difference $(\Delta \rho)$ of interdendritic liquid. It is depends on the composition difference and temperature difference of the alloy.

2) Primary and secondary dendrite arm spacings $\left(d_{1}\right.$ and $\left.d_{2}\right)$.

3) Fraction of liquid $\left(f_{L}\right)$.

4) Solidification front angle $(\theta)$.

It is noted that processing conditions are another important control parameter that was not studied in detail in the current research. Composition effects on the key control parameters will be thoroughly investigated in chapter 4 and chapter 5 using numerical and 
experimental approaches. In chapter 6, the key control parameters will be incorporated into the freckling criterion developed.

\subsection{Summary}

The results and discussion in this chapter are summarized as follows:

1. Freckles in alloy 625 are significantly enriched with $\mathrm{Nb}$ and Mo.

2. Sharp boundary on the solidus side and diffuse boundary on the liquidus side are observed for freckles in 625. Freckles contain large amount of Laves and $\delta-\mathrm{Ni}_{3} \mathrm{Nb}$.

3. Freckle formation in model alloys has been investigated in physical simulation using horizontal solidification. Freckles are generated in all of the three model alloys.

4. In alloy HV8851 (Ni-Cr-Ti), freckles initiate at the bottom of the ingot and flow upward during solidification.

5. In alloys HV8852 (Ni-Cr-Nb) and HV8853 (Ni-Cr-Fe-Nb), freckles originate at the top of ingots and flow downwards.

6. 2 freckle channels are seen on one half of the ingot cross section of HV8852, while 15 are found in HV8853. The number of freckles cannot be obtained in HV8851 due to the poor contrast, however, freckles occurrence is prevalent in the alloy.

7. Solidification front angles are 50, 40, and 30 degrees for alloys HV8851, HV8852, and HV8853 respectively.

8. Diffuse boundary on the liquidus side and sharp boundary on the solidus side are observed in the freckles of all the alloys.

9. Freckling mechanism in horizontal solidification is proposed. The physical simulation can be applied to freckling study in remelting of superalloys.

10. Key control parameters for freckling are identified to be the liquid density difference, the dendrite arm spacings, the solidification front angle, and the fraction of solid (or liquid) during solidification. 

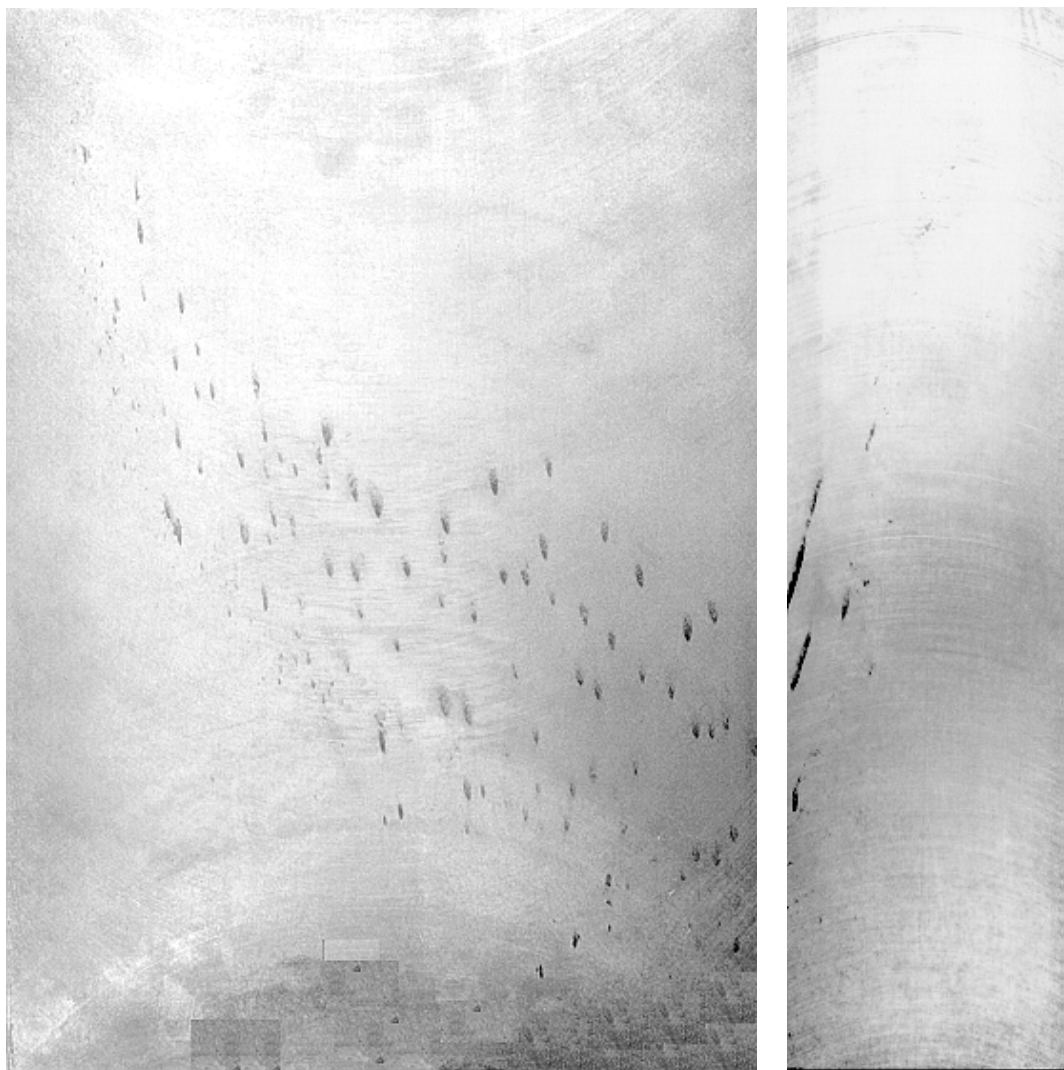

Figure 3-1: Cut-up surfaces showing freckles decorating pool profiles.
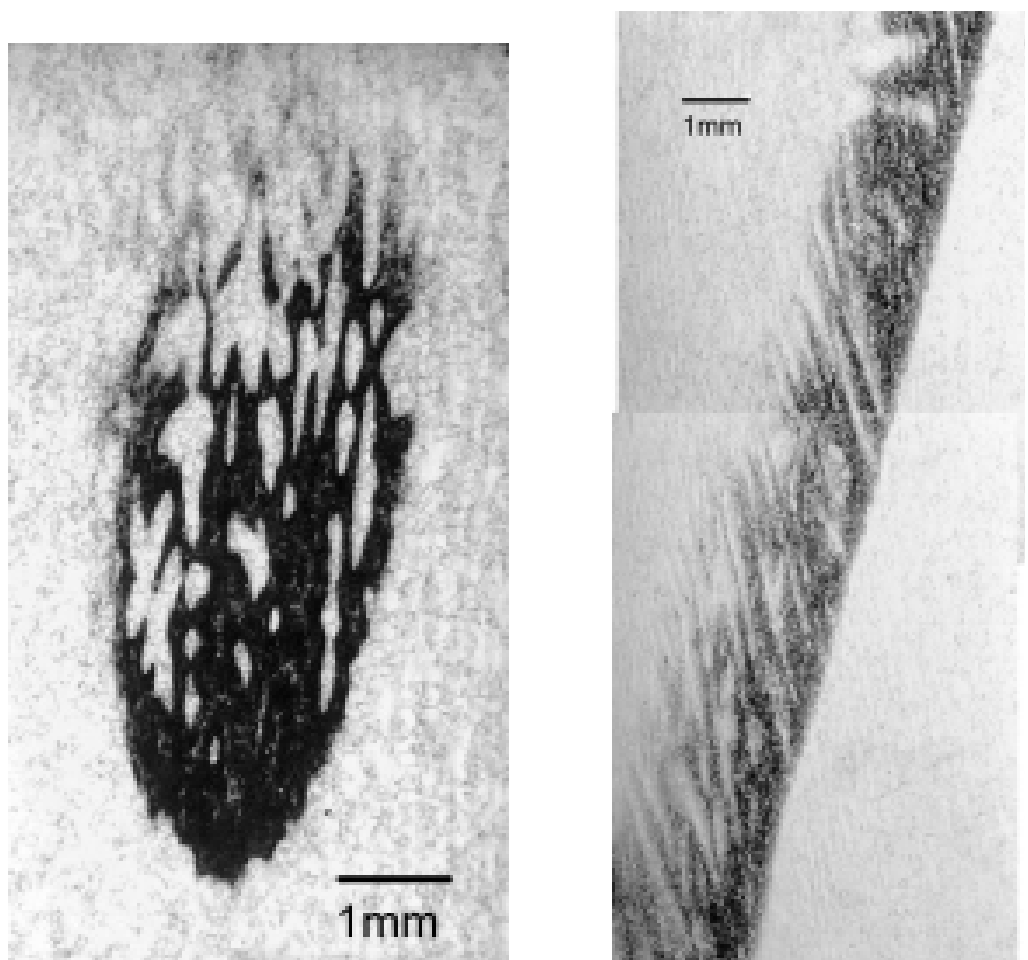

Figure 3-2: Macrostructure of transverse (left) and longitudinal (right) sections of freckles. 

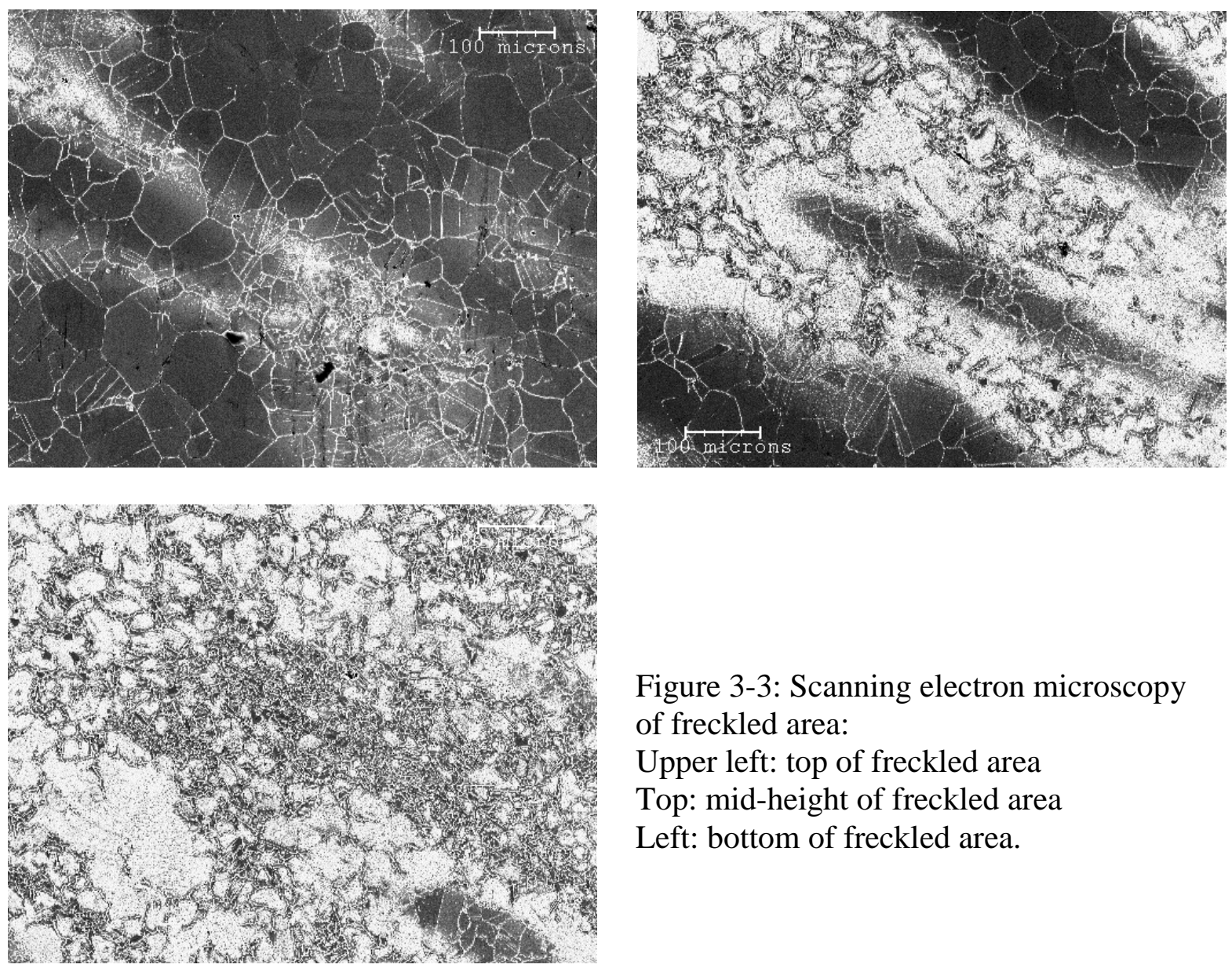

Figure 3-3: Scanning electron microscopy of freckled area:

Upper left: top of freckled area

Top: mid-height of freckled area

Left: bottom of freckled area.

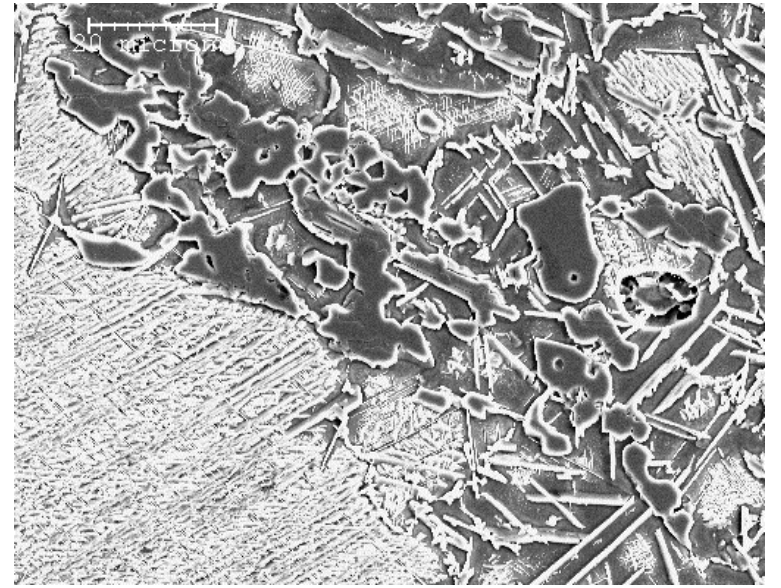

(a)

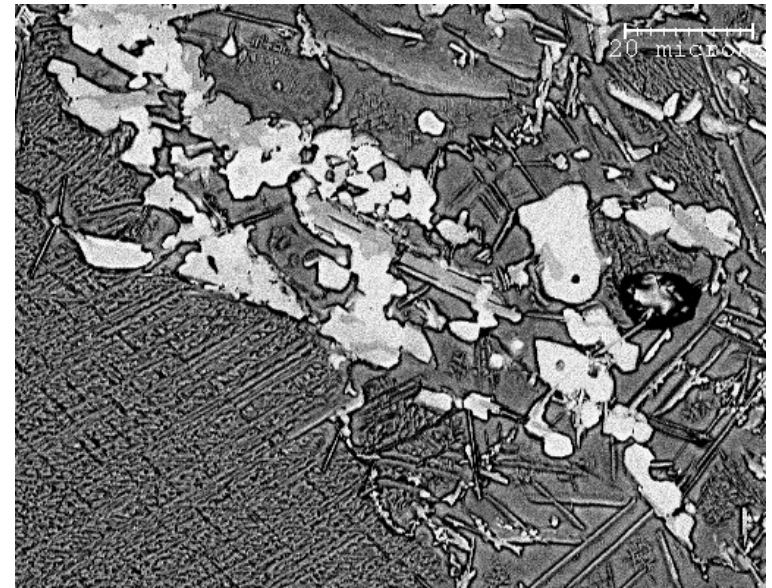

(b)

Figure 3-4: Segregation and phases in freckled area of alloy 625. (a) secondary electron image; (b) back-scattered electron image of the same field from (a). 


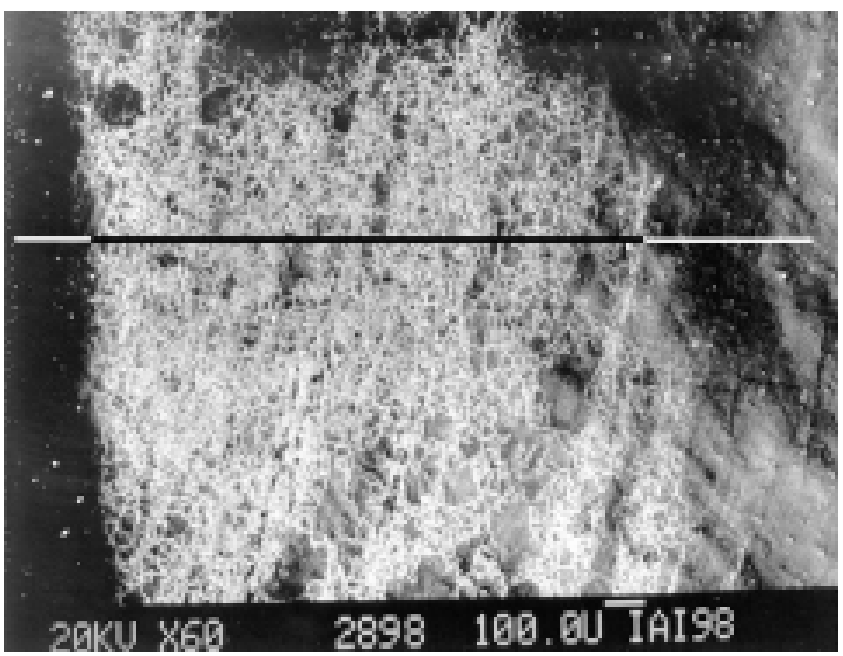

(a)

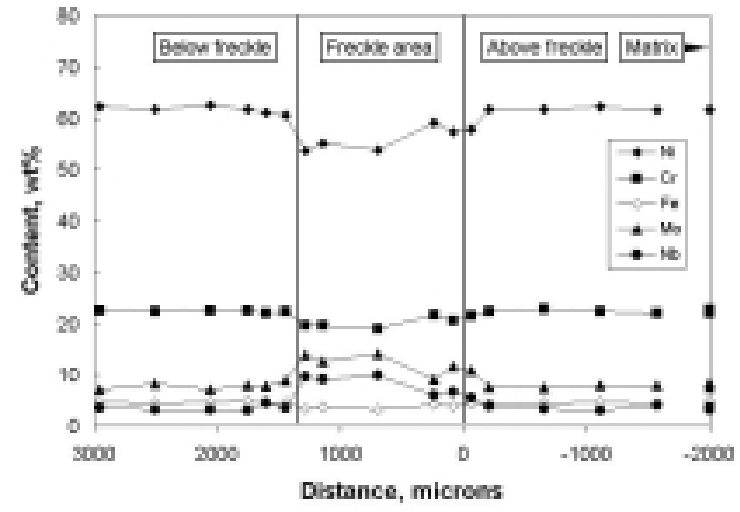

(b)

Figure 3-5: Quantitative EDS analysis on the line-scan across the freckled area. 


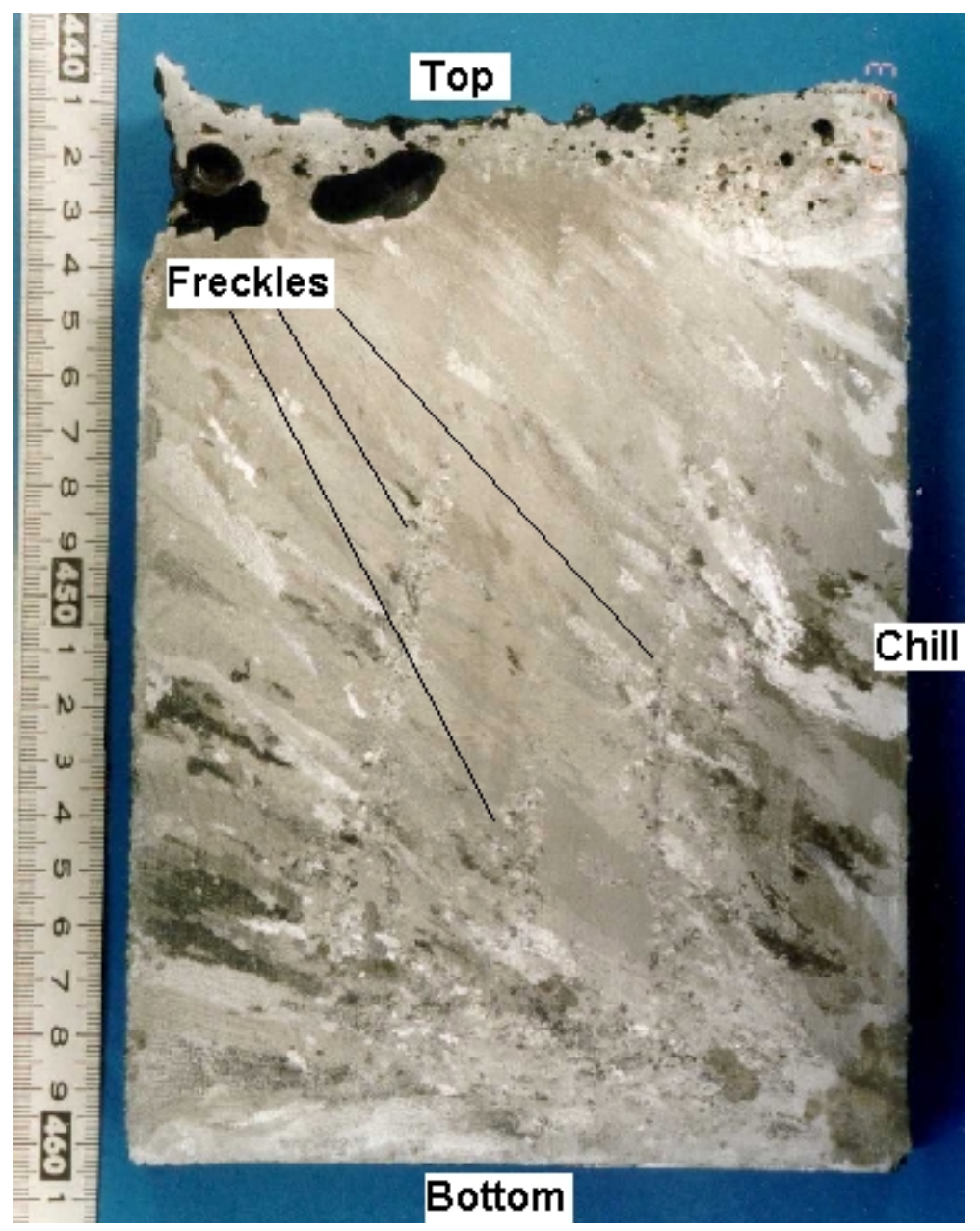

Figure 3-6 (a): Longitudinal cross section of HV8851 revealing freckles. (Chill side is on the right. Gravity force pointing downward.) 


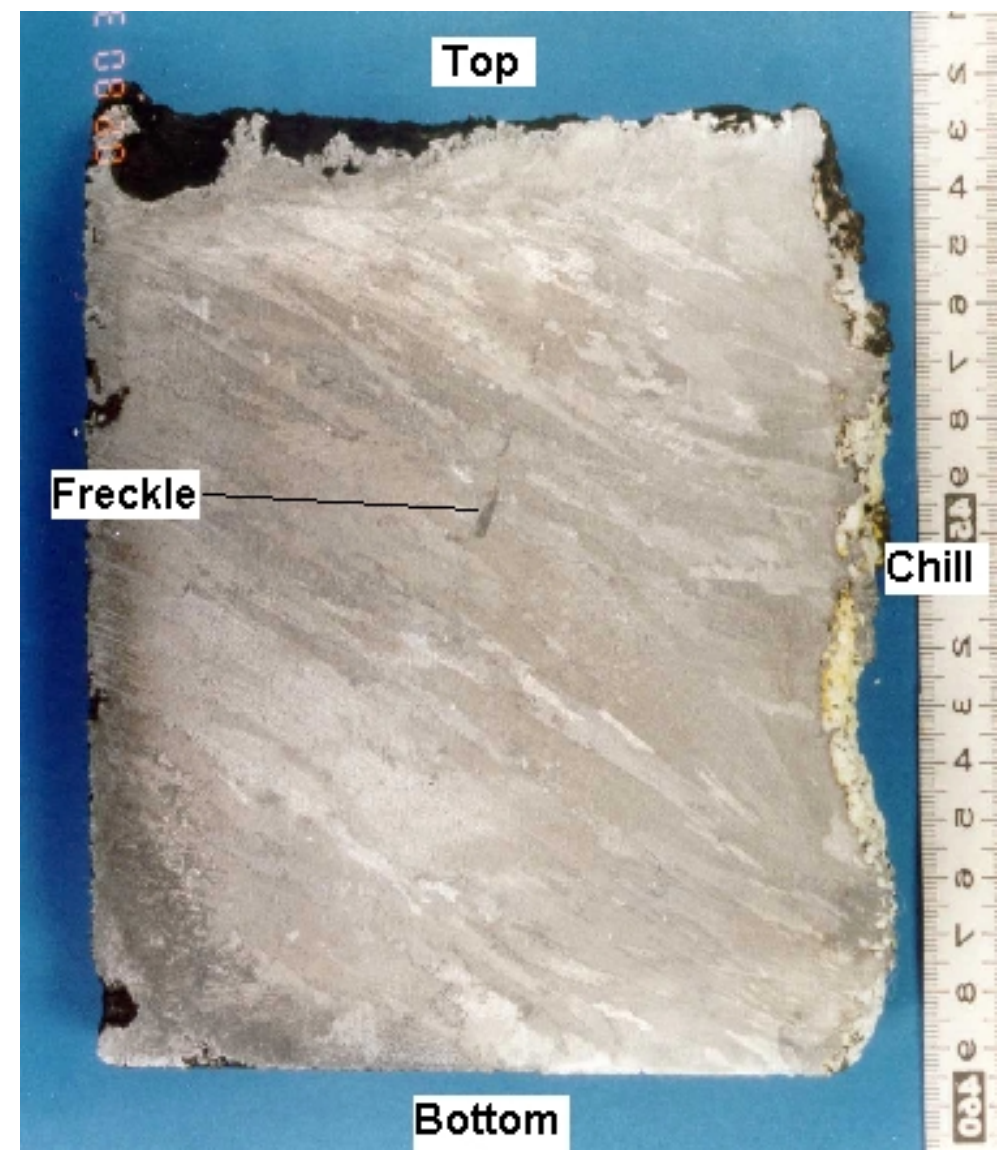

Figure 3-6 (b): Longitudinal cross section of HV8852 revealing freckles. (Chill side is on the right. Gravity force pointing downward.) 


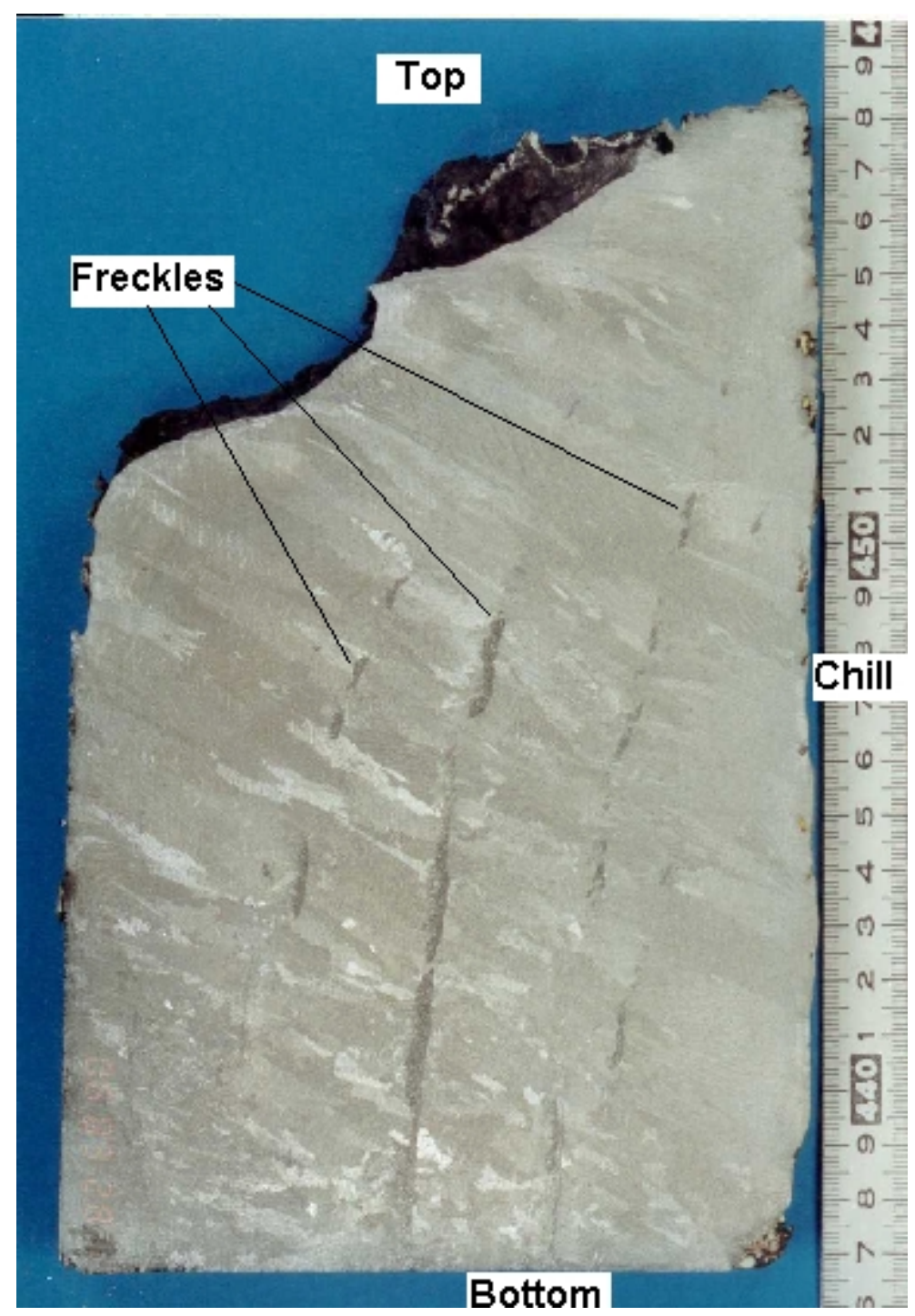

Figure 3-6 (c): Longitudinal cross section of HV8853 revealing freckles. (Chill side is on the right. Gravity force pointing downward.) 


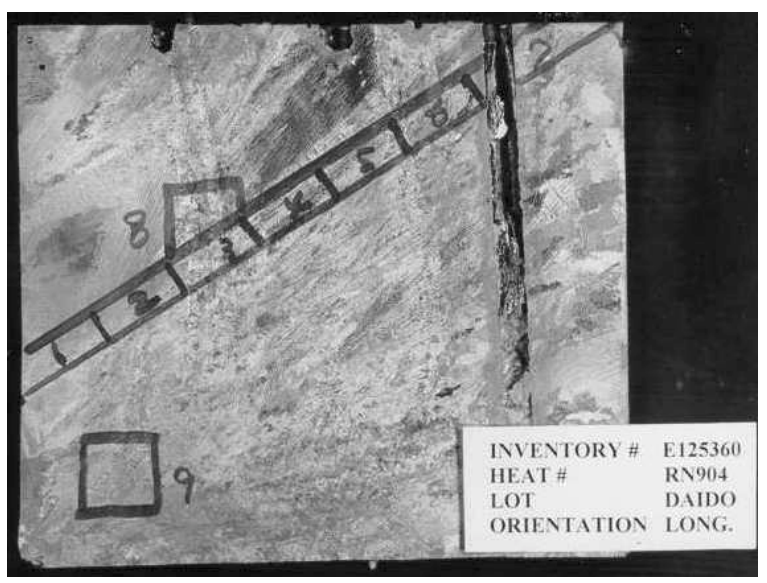

(a)

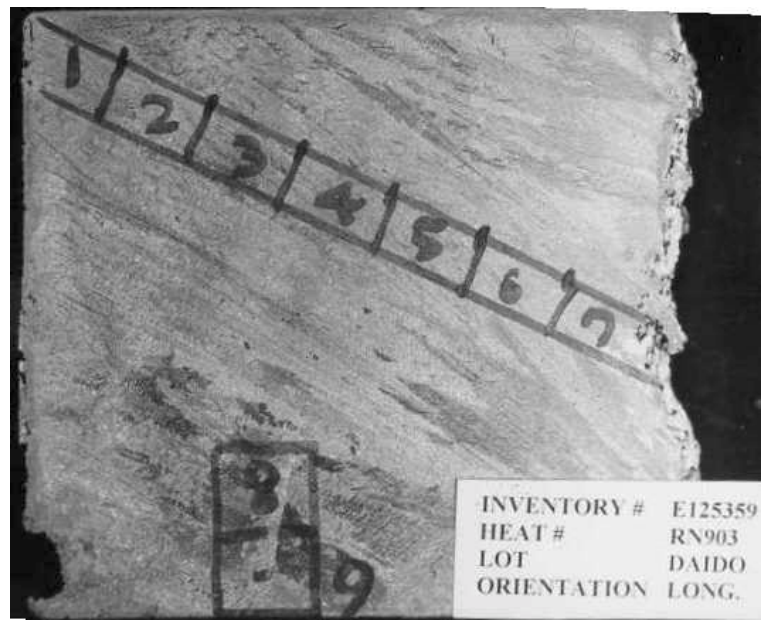

(b)

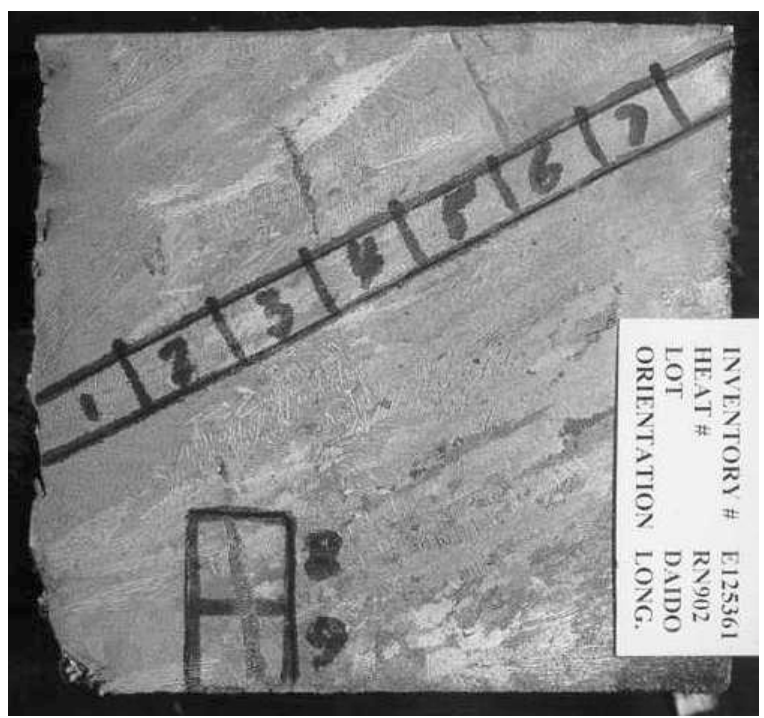

(c)

Figure 3-7: Cut-up plans for (a) HV8851, (b) HV8852, and (c) HV8853. Photos show the longitudinal cross section and $\mathrm{d} 2$ were measured on the transverse cross sections. 


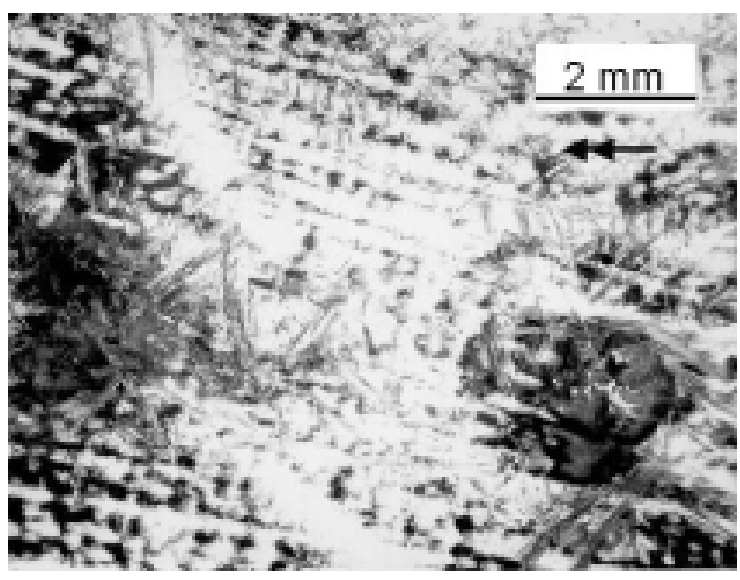

(a)

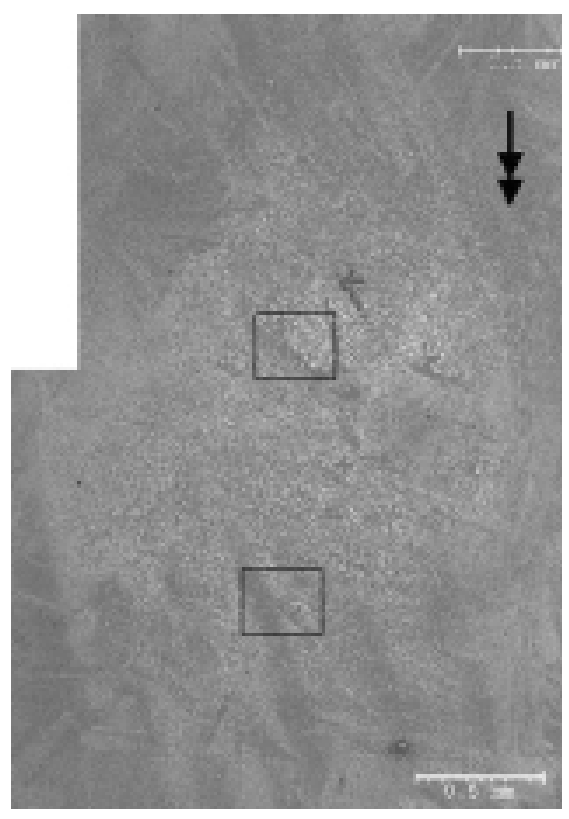

(b)

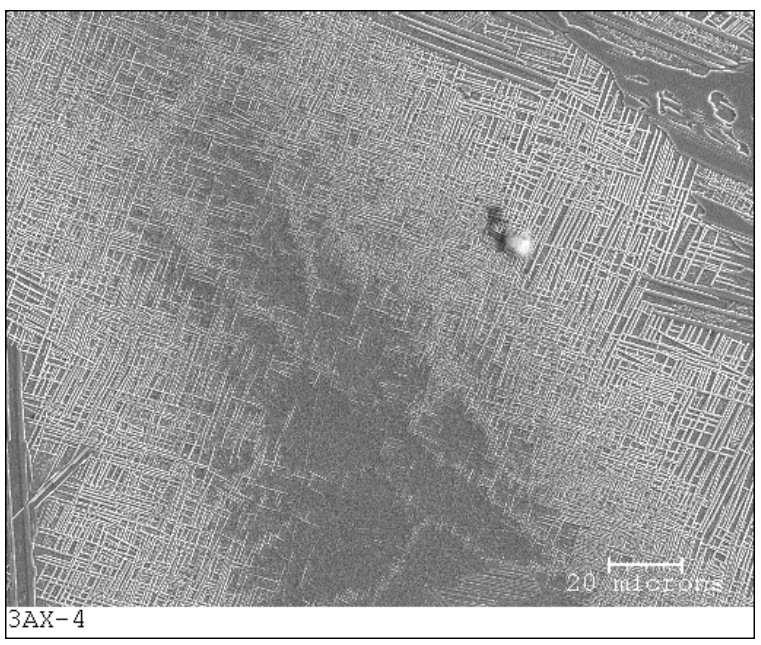

(c)

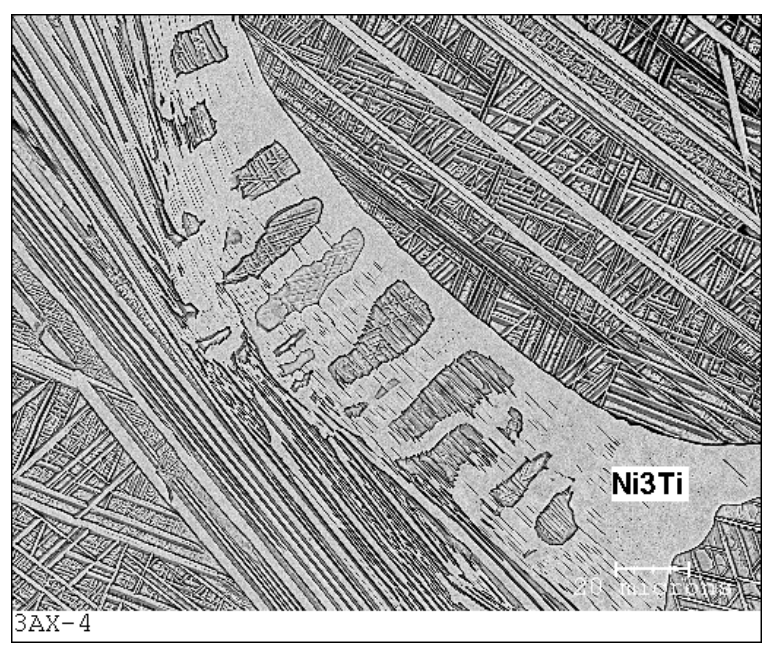

(d)

Figure 3-8: Freckles in HV8851. (a) Optical microphotograph (double arrow indicates the growth direction); (b) shows the secondary electron images of one freckle in (a); (c) is the enlarged area of the lower region in (b); (d) is the back-scattered electron image of the enlarged area of the upper region in (b). 


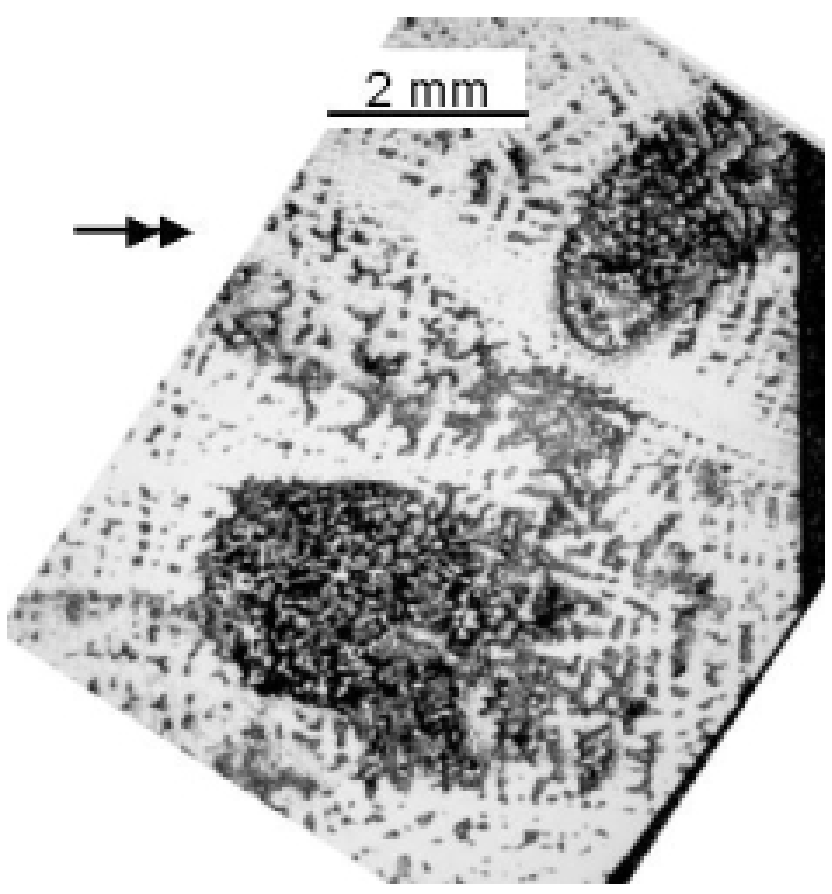

(a)

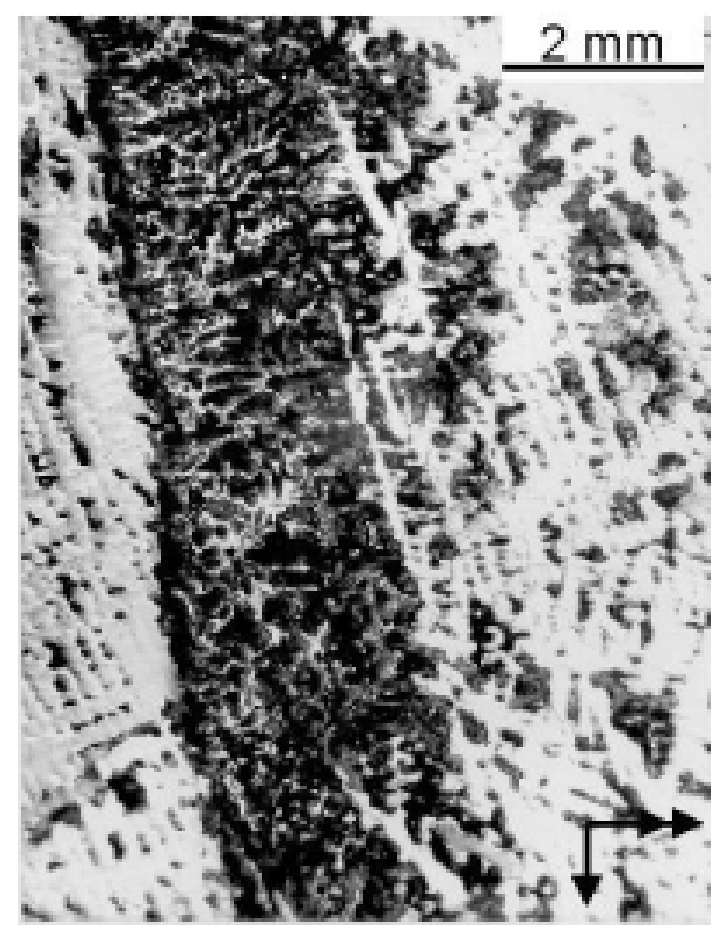

(b)

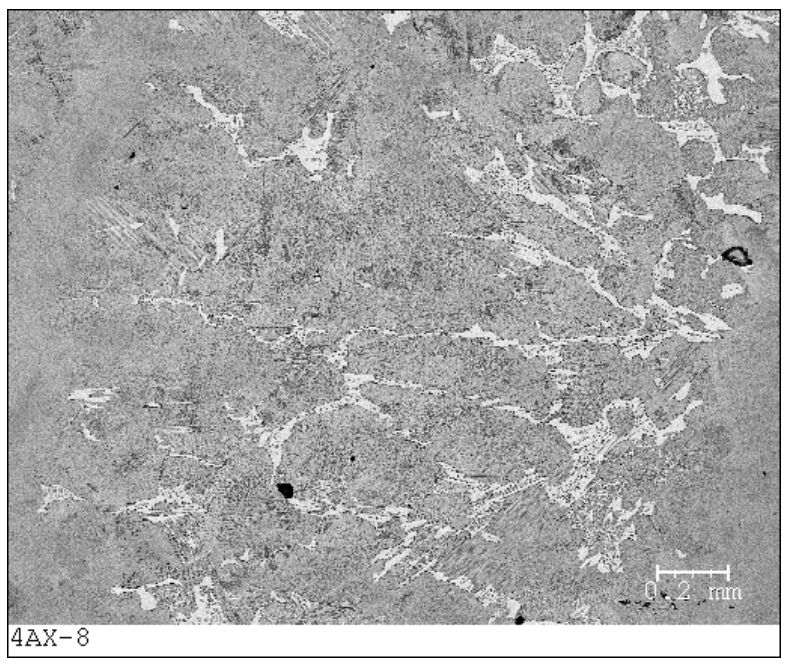

(c)

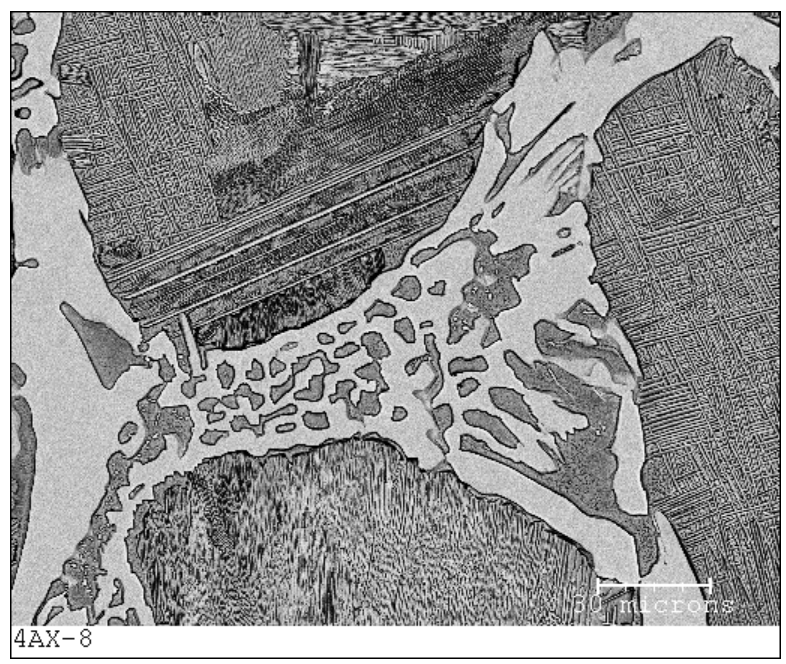

(d)

Figure 3-9: Optical photomicrographs of (a) transverse and (b) longitudinal cross sections of freckles in HV8852. (Double arrow is the growth direction and single arrow is gravity direction). (c) is the SEM image of longitudinal section of freckles, solidus side is on the left. (d) is the back-scattered electron image of eutectic constituents in freckled area. 


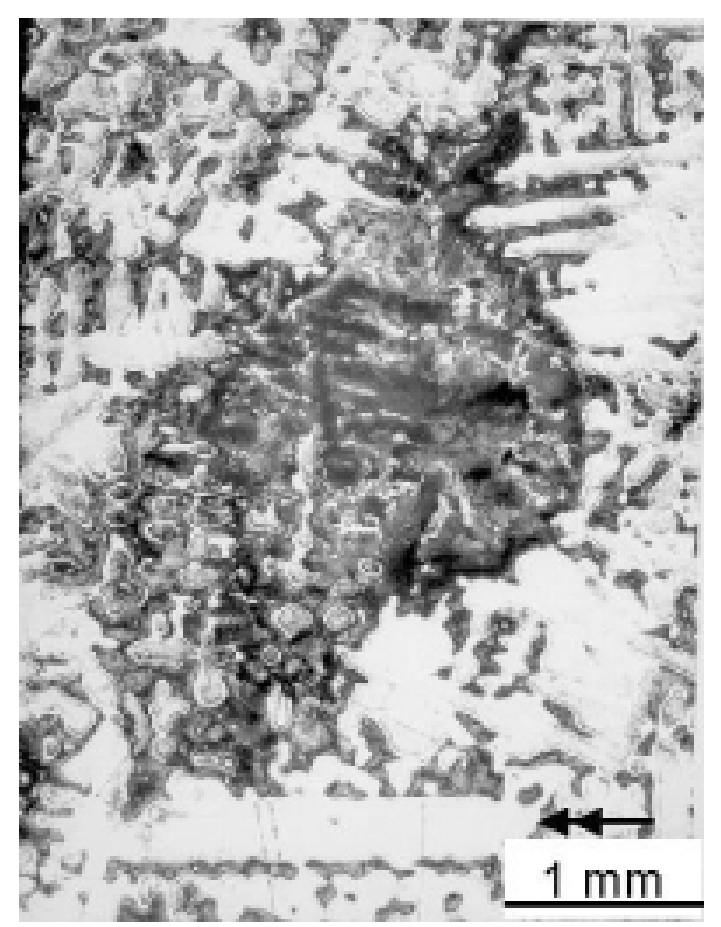

(a)

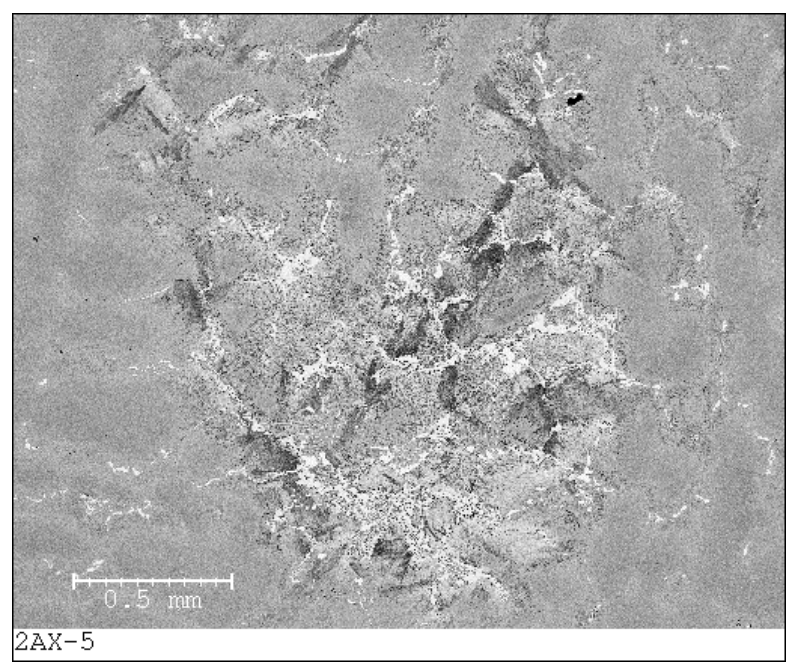

(c)

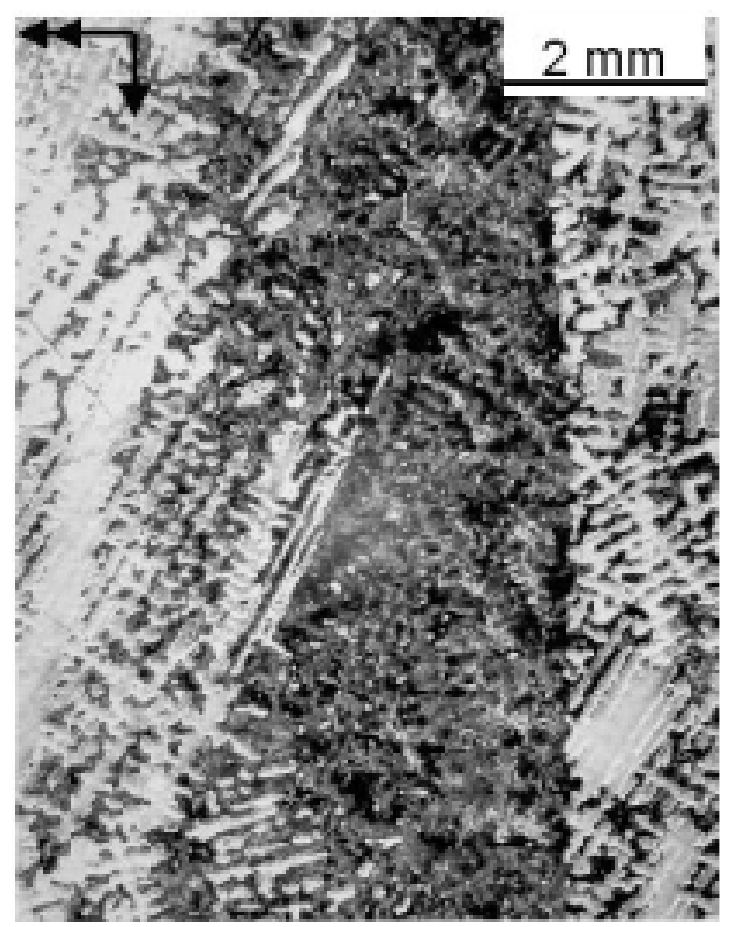

(b)

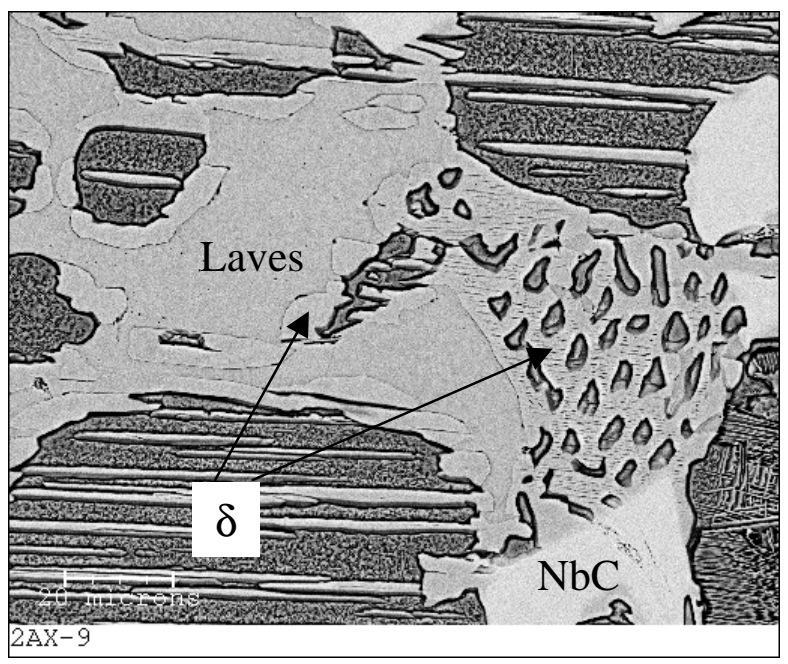

(d)

Figure 3-10: Optical photomicrographs of (a) transverse and (b) longitudinal cross sections of freckles in HV8853. (Double arrow is the growth direction and single arrow is gravity direction). (c) is the SEM image of transverse cross section of freckle (growth direction points upward.) (d) shows the eutectic constituents in freckled area. 


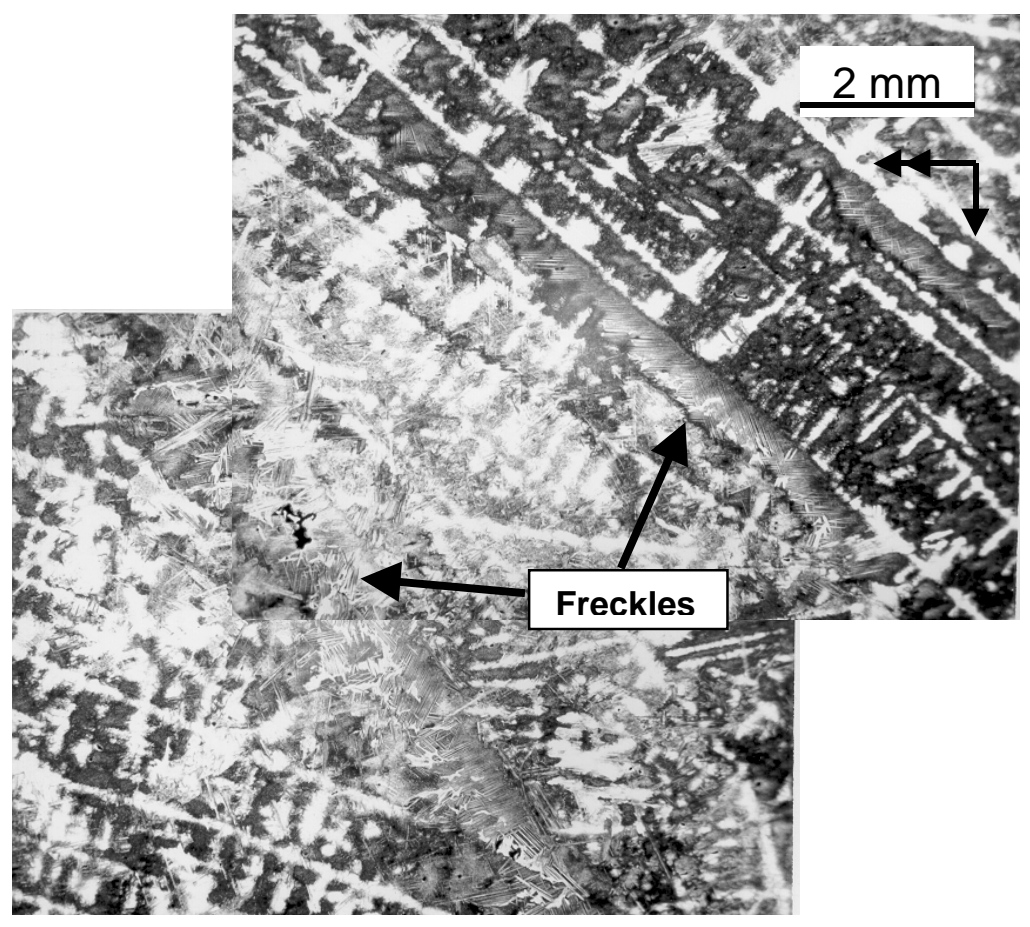

Figure 3-11: Freckle initiation in HV8851. Optical photomicrograph shows two freckle trials originating from bottom of the ingot.

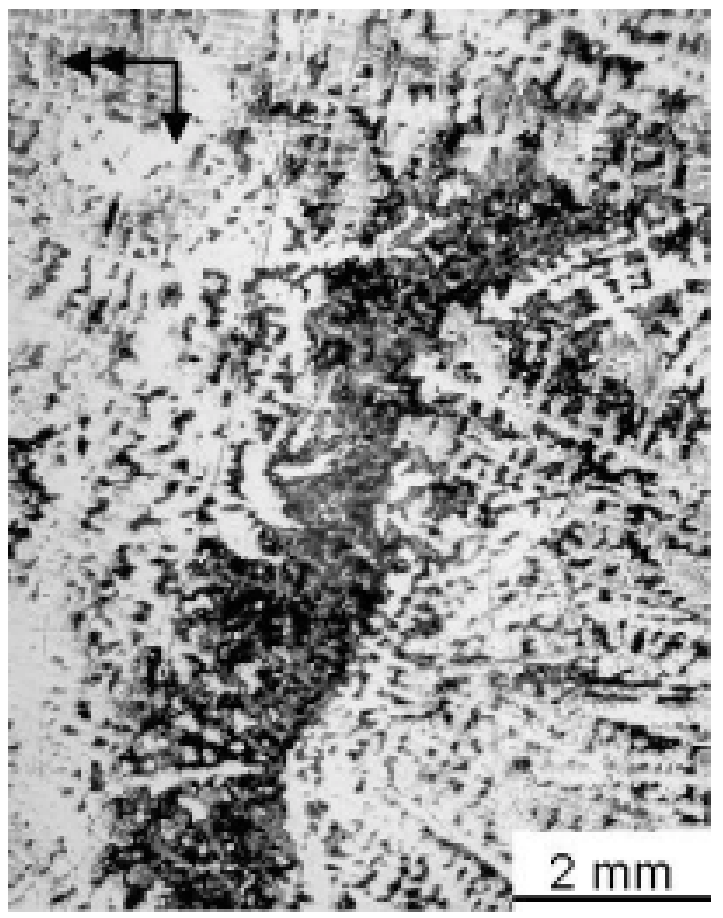

Figure 3-12: Longitudinal section of freckles in HV8853 showing the irregularities of dendrites and downward freckle flow. (Gravity force points downwards and ingot grows to the left) 


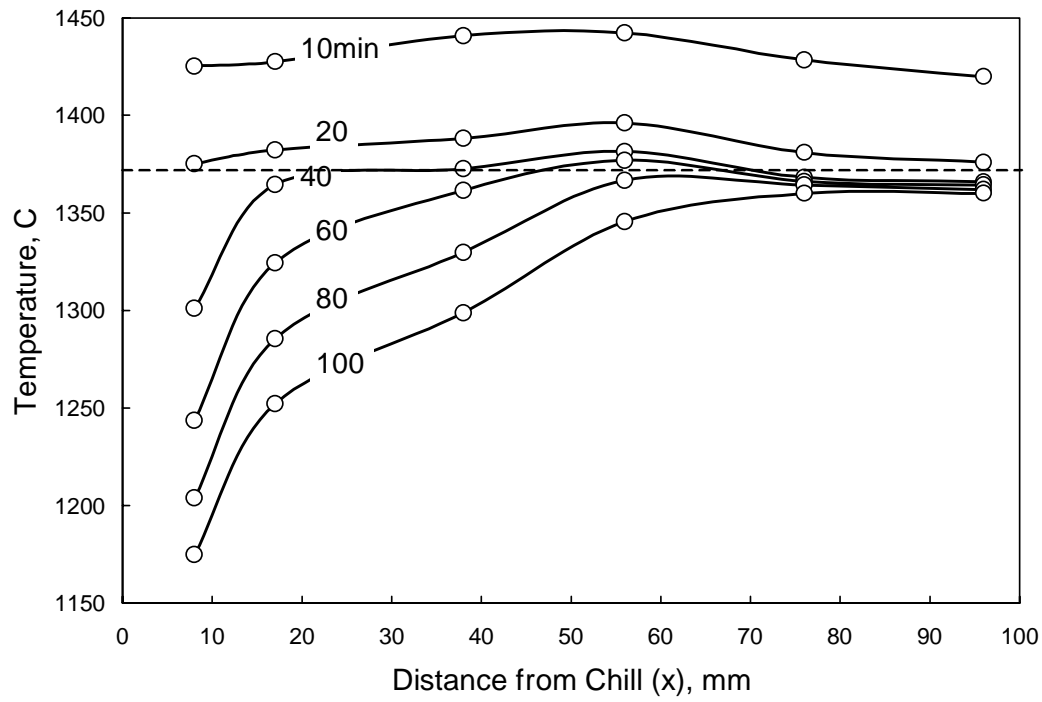

(a)

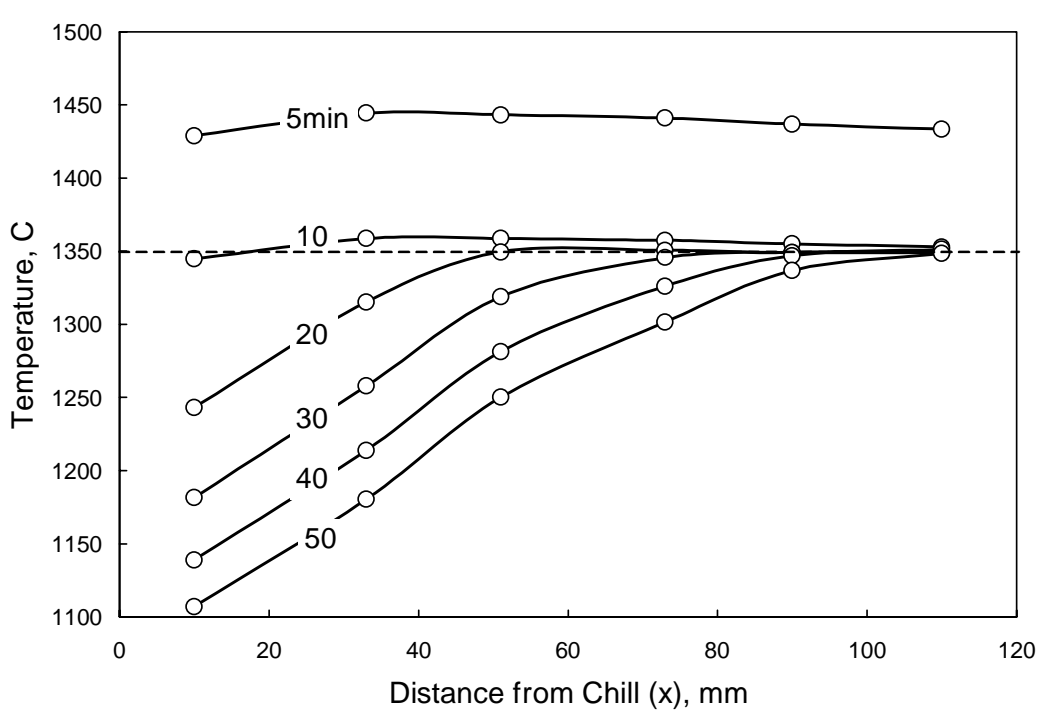

(c)

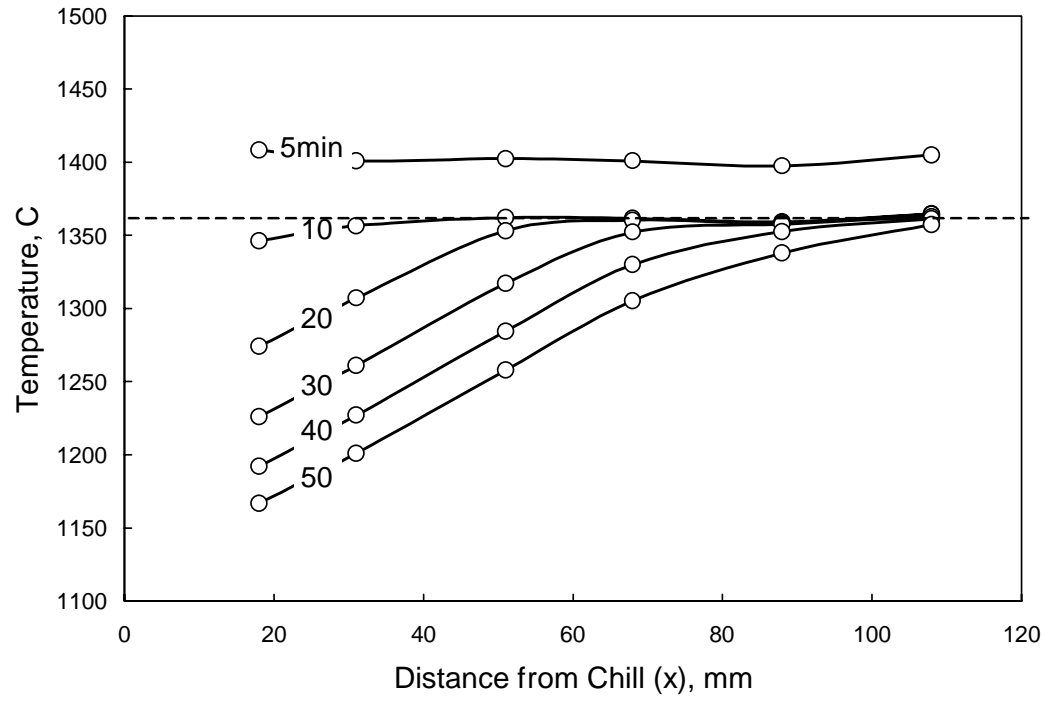

(b)

Figure 3-13: Temperature profiles of (a) HV8851 (Liq. $=1373^{\circ} \mathrm{C}$ ), (b) $\mathrm{HV} 8852\left(\mathrm{Liq} .=1364^{\circ} \mathrm{C}\right.$ ), and (c) HV8853 (Liq. $=1351^{\circ} \mathrm{C}$ ). Dash lines are the liquidus temperatures of the model alloys. 


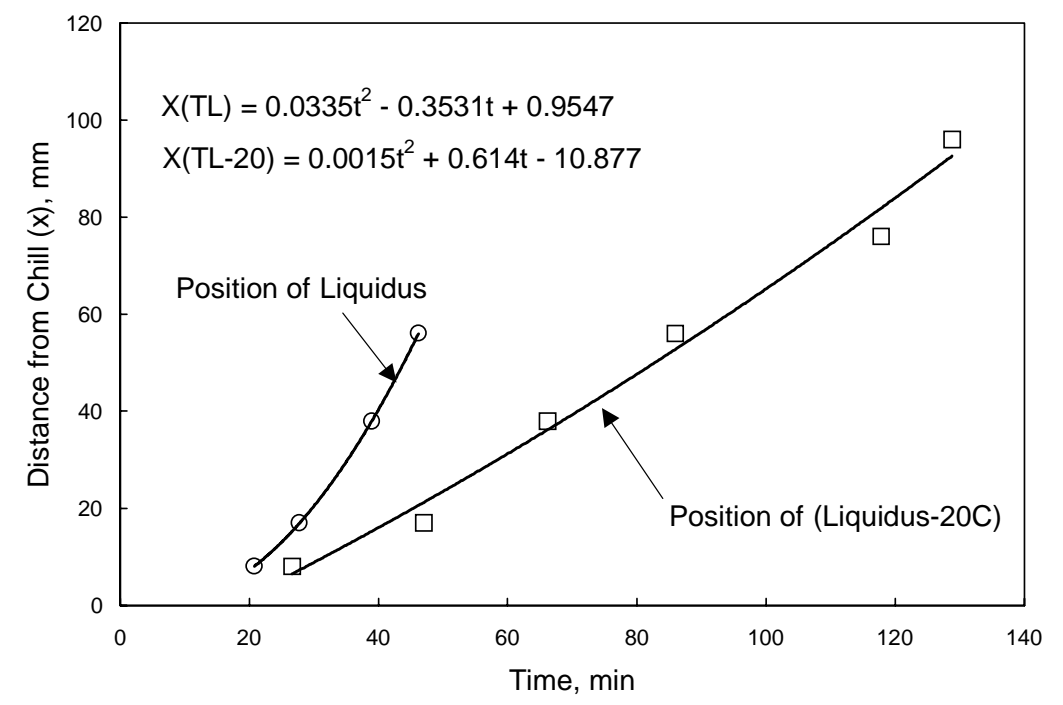

(a)

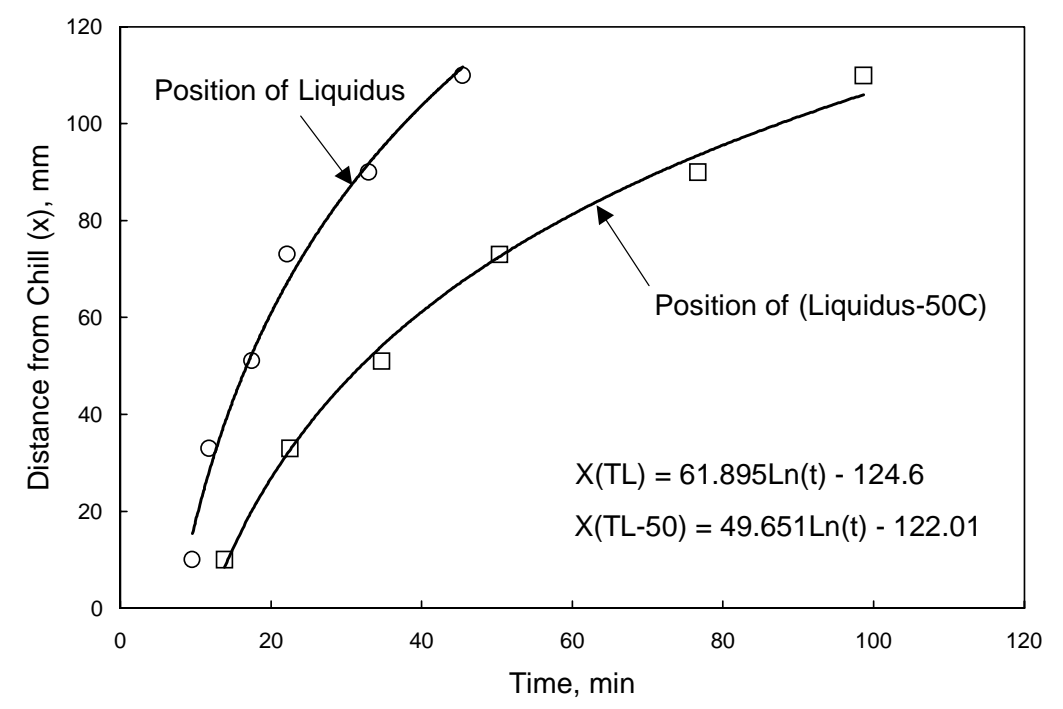

(c)

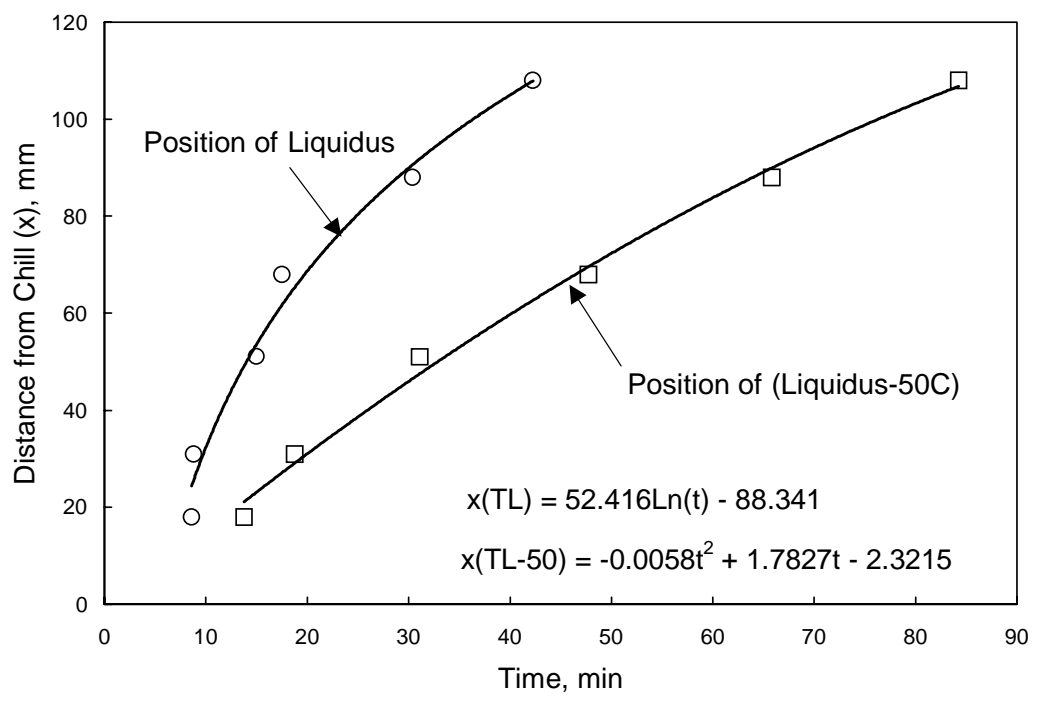

(b)

Figure 3-14: Positions of liquidus and isotherms that are $50^{\circ} \mathrm{C}\left(20^{\circ} \mathrm{C}\right)$ below liquidus of (a) HV8851, (b) HV8852, and (c) HV8853. 


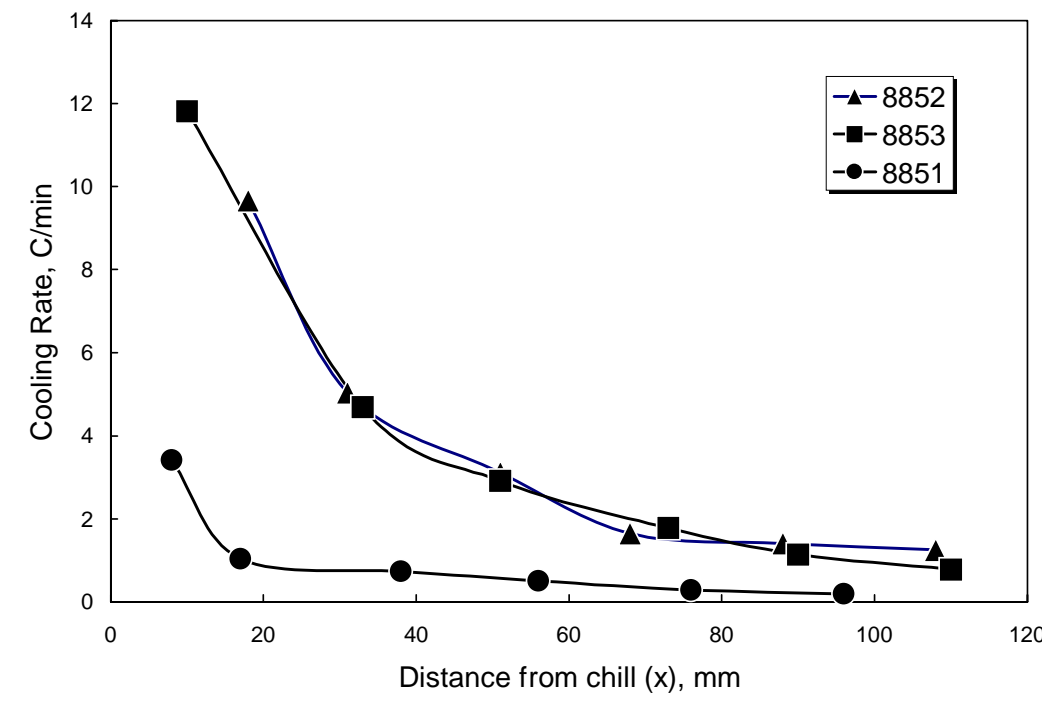

(a)

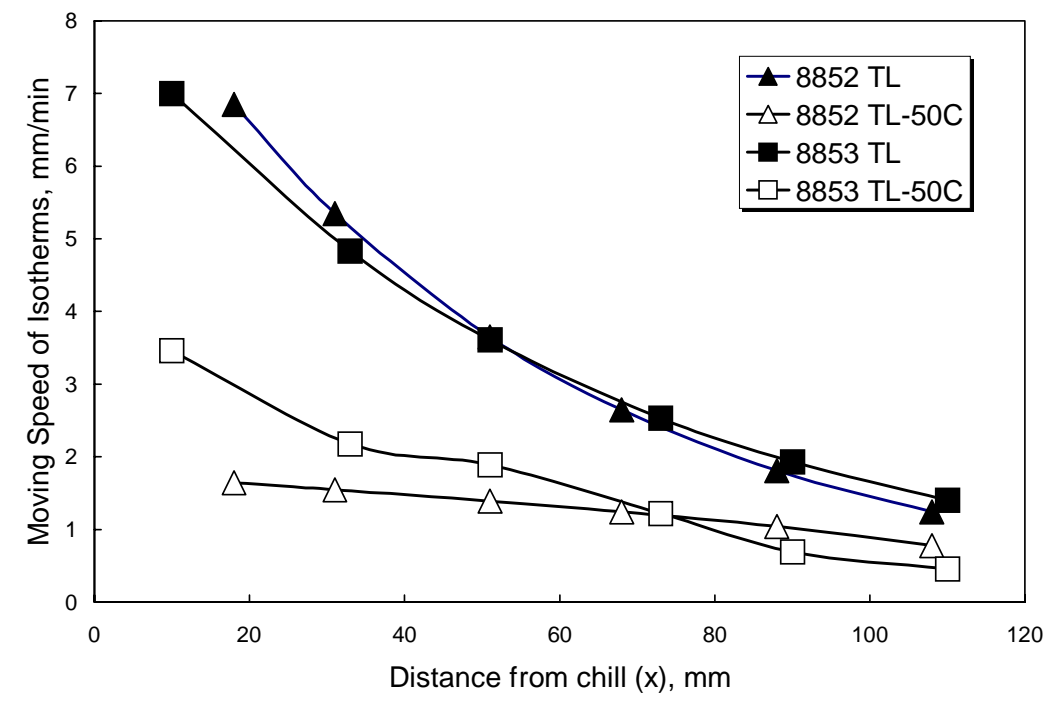

(c)

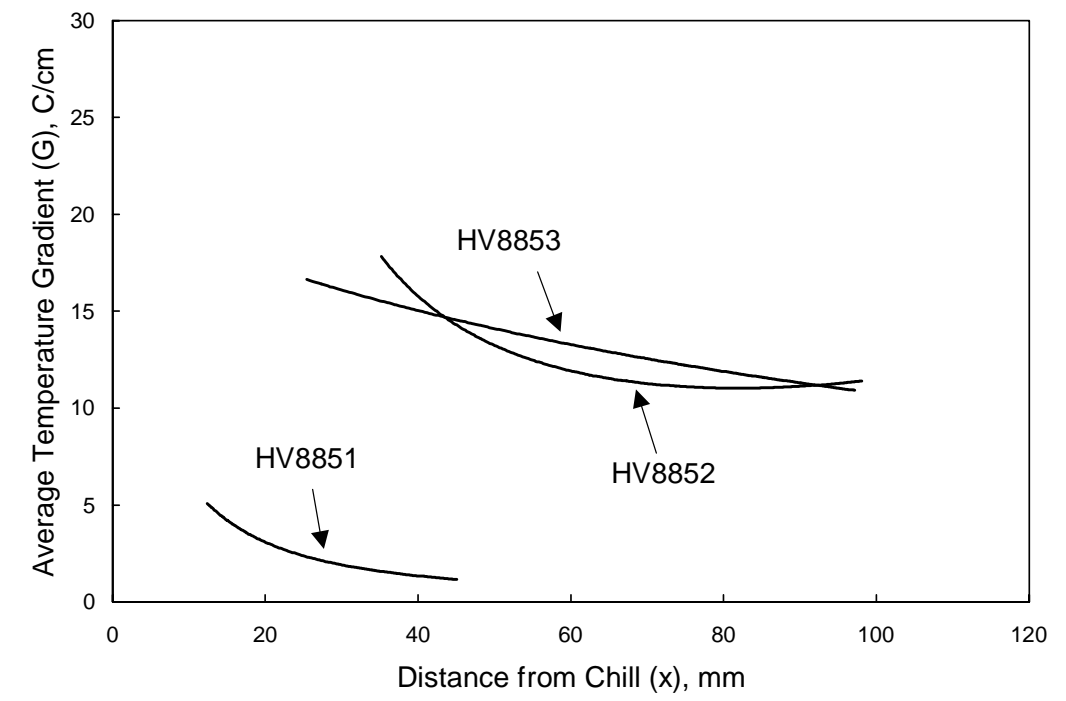

(b)
Figure 3-15: (a) Cooling rate, (b) thermal gradient, and (c) moving speeds of model alloys during horizontal solidification. 


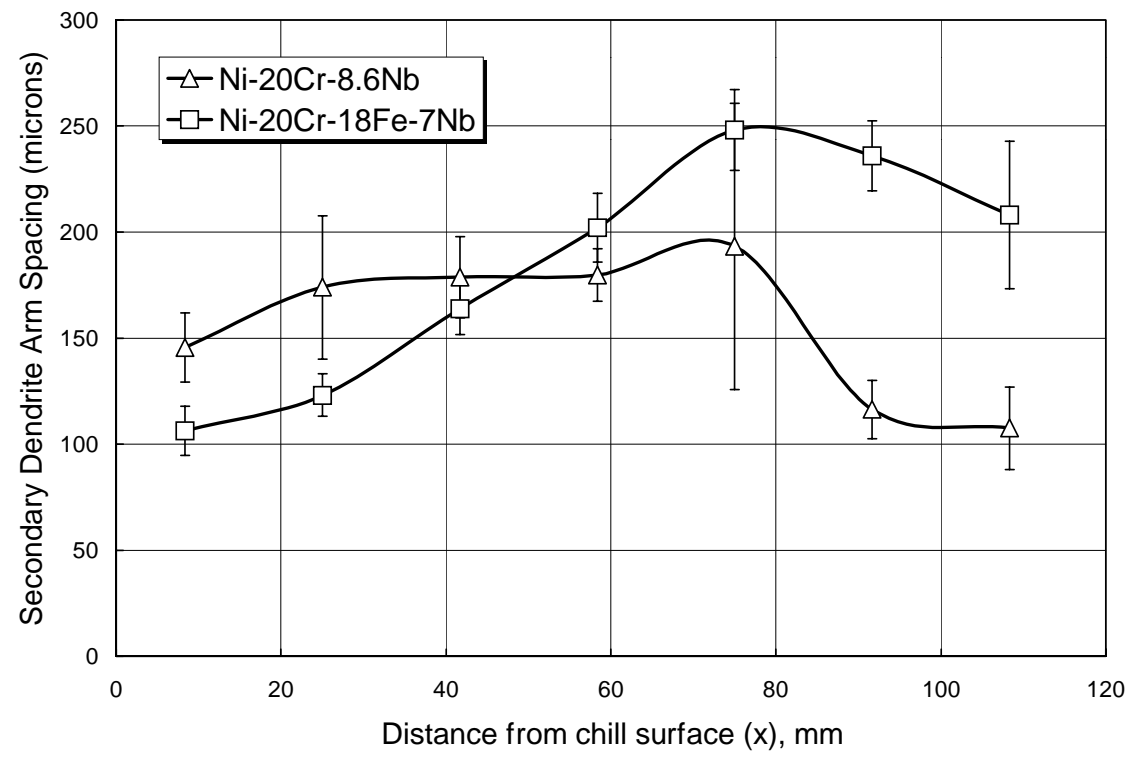

Figure 3-16: Secondary dendrite arm spacings versus distance from chill in horizontally solidified model alloys (HV8852: Ni-20Cr-8.6Nb and HV8853: Ni-20Cr-18Fe-7Nb).
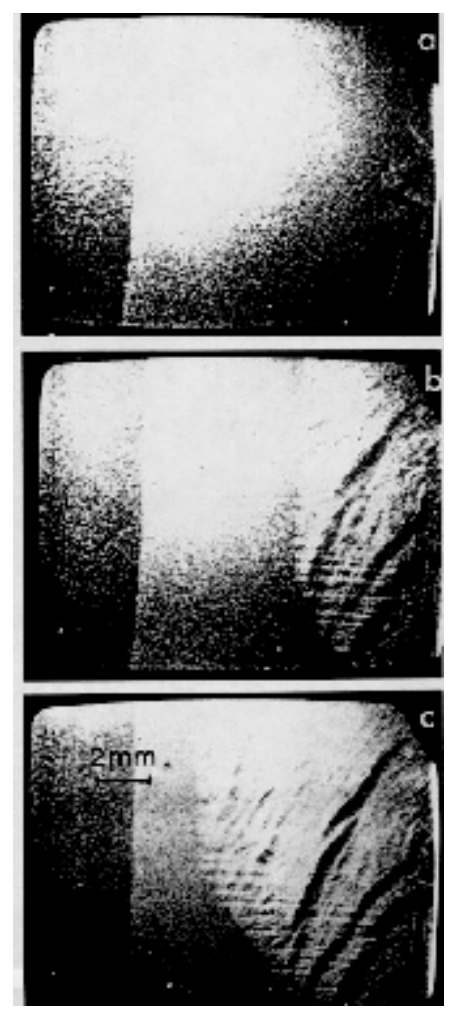

Figure 3-17: Horizontal solidification of binary Al-20Cu at different intervals during solidification. Chill is on the right hand side and crystal grows from right to left [44]. 

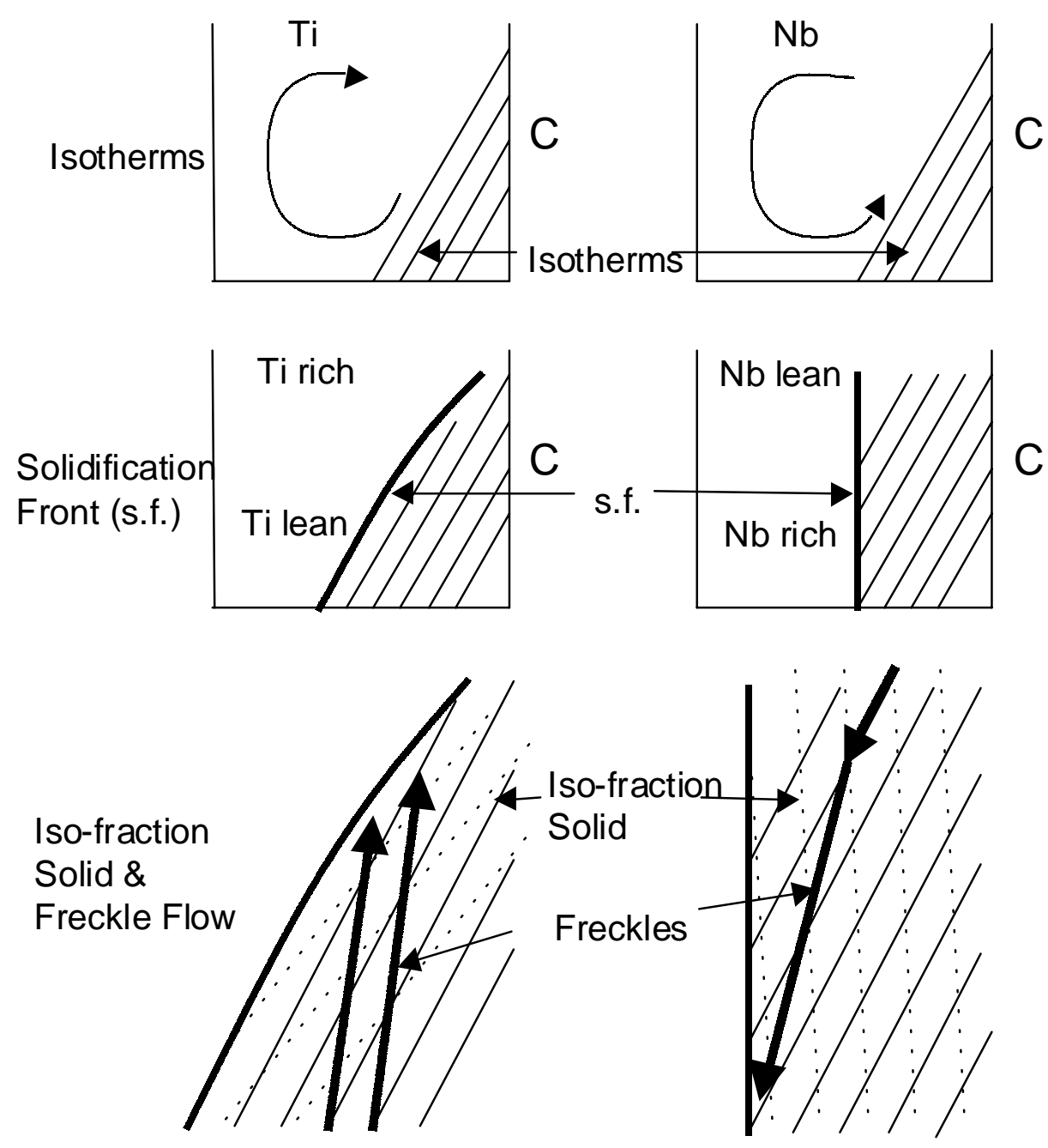

(a)

(b)

Figure 3-18: Schematics of isotherms, solidification front, iso-fraction solid and freckle flow in the mushy zone of horizontal solidification of (a) Ti-containing and (b) Nb-containing model alloys. In Ti-containing alloy, iso-fraction solids are parallel to isotherms.

("C" stands for chill surface, "s.f." is the solidification front.) 


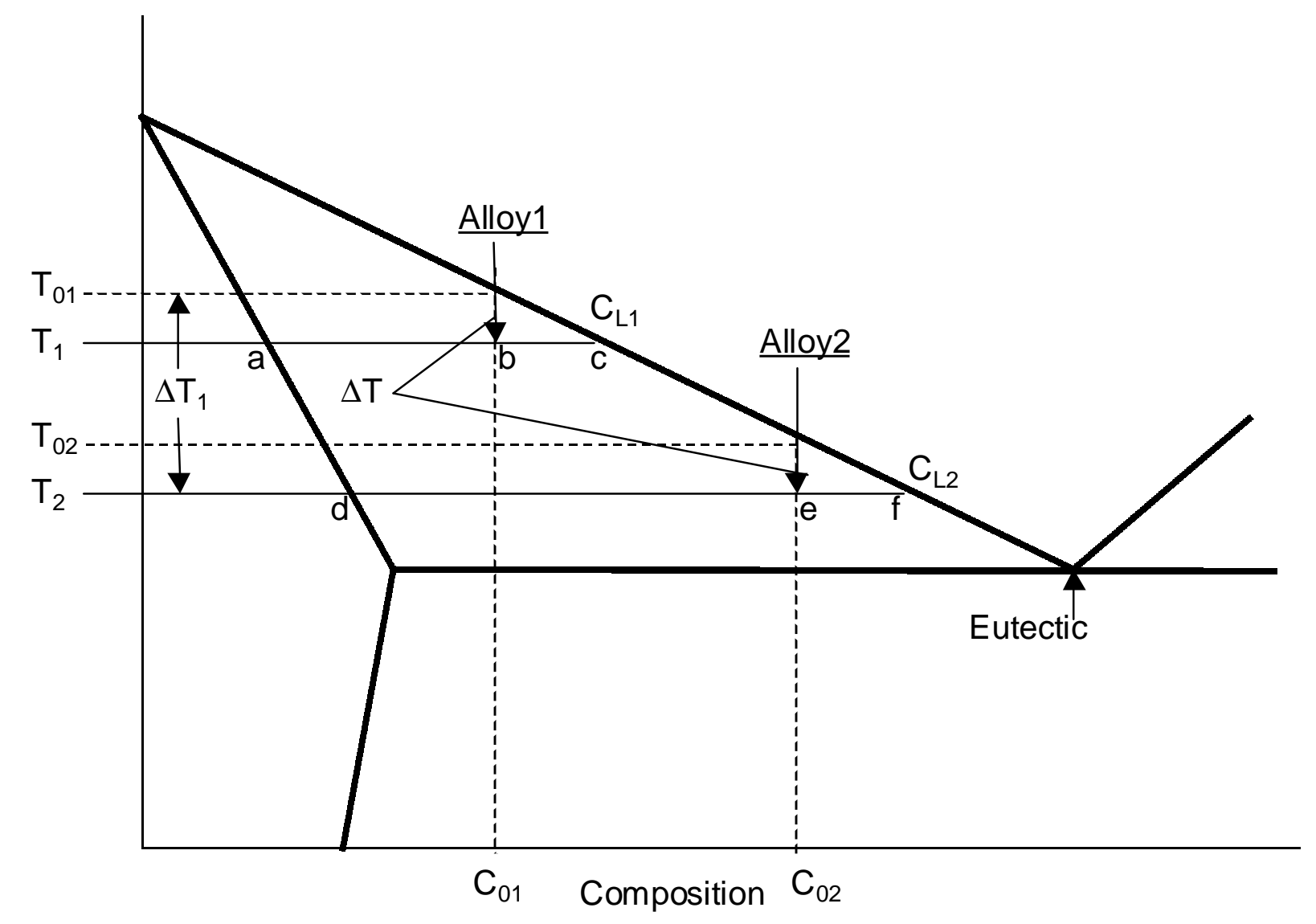

Figure 3-19: Schematic phase diagram shows that for two liquid alloys and the same temperature drop from liquidus, the fraction of solid of alloy 1 is greater than that of alloy 2 . Here the fraction of solid is defined as the ratio of bc/ac for alloy 1 , and ef/df for alloy 2 . Since bc/ac > ef/df, fraction solid of alloy 1 is greater than alloy 2 . 
Chapter 4

\section{Solidification in Ni-based Model Superalloys: CALPHAD Approach}

4.1. Introduction 66

4.2. Approaches 66

4.2.1. Model Superalloys 66

4.2.2. CALPHAD method in Solidification Simulation 67

4.3. Solidification Diagram of Ni-Cr-Fe-Nb System 69

4.3.1. Ternary Systems $\quad 69$

4.3.2. Quaternary System: CALPHAD Approach 70

4.3.3. Experimental Results 70

4.3.4. Alloying Effects 72

4.4. Freezing Range of Model Alloys 73

4.4.1. Freezing Range 74

4.4.2. Alloying Effects $\quad 75$

4.5. Solidification Sequence and Secondary Phases 76

4.5.1. Solidification Sequence 76

4.5.2. Secondary Phases 77

$\begin{array}{ll}\text { 4.5.3. Alloying Effects } & 78\end{array}$

$\begin{array}{lll}\text { 4.6. Summary } & 79\end{array}$ 


\subsection{Introduction}

Freckle segregates in solidification is a macroscopic phenomenon that is intimately associated with the alloy of interest. The key control parameters of freckling, which were identified in chapter 3, can be traced back to the solidification metallurgy that is independent of processing conditions. However, there is insufficient data available to define the solidification behavior of these multicomponent alloys. When working with such highly alloyed materials, information is based purely on empirical knowledge. This chapter will study the fundamental solidification metallurgy in model superalloys. The objective is to develop renovated models that can be applied in microscopic and macroscopic segregation studies.

\subsection{Approaches}

\subsubsection{Model Superalloys}

Industrial Ni-based superalloys contain $\mathrm{Ni}, \mathrm{Cr}, \mathrm{Co}$ and $\mathrm{Fe}$ as the matrix-forming elements, and additions of $\mathrm{Nb}, \mathrm{Ti}, \mathrm{Al}$ and $\mathrm{Mo}$ to promote solution and precipitation hardening. In contrary to their benefits in improving mechanical properties, these solutes have pronounced effects on the solidification structure. First, these alloys are prone to the formation of freckle defects in remelting when certain levels of solutes were added. Second, secondary phases form at the late stage of solidification. These incoherent phases can cause incipient melting in forging and welding due to their low melting temperatures. They also consume significant amounts of solutes that are needed for the precipitation of strengthening coherent phases. Therefore, it is critical to define the influences of individual alloying element and their interactions on the final ingot structure. This is realized by the design of model superalloys that contain only the vital few alloying elements. In this study, four model alloy systems are selected to investigate the alloying effects and the interactions of $\mathrm{Cr}, \mathrm{Fe}, \mathrm{Nb}, \mathrm{Ti}$ and $\mathrm{Al}$.

$\mathrm{Ni}-\mathrm{Cr}-\mathrm{Fe}-\mathrm{Nb}$ system is chosen as the first example to study the alloying effects of $\mathrm{Cr}, \mathrm{Fe}$, and $\mathrm{Nb}$. The quaternary system represents the simplest, yet the most fundamental case of the complex Nb-containing superalloys. It is generally accepted that the 
segregation of $\mathrm{Nb}$ dominates the solidification sequence in these alloys. Thus, this model alloy system focuses on the $\mathrm{Nb}$ effect by excluding the interactions of other solute elements. Both equilibrium and Scheil modules in Thermo-Calc were employed to simulate the solidification behaviors in a series of model alloys. The results are validated by observations of experimental $\mathrm{Ni}-\mathrm{Cr}-\mathrm{Fe}-\mathrm{Nb}$ alloys. A pseudo-solidification diagram is established to explain the alloying effects on solidification sequence and secondary phases.

Based on the comprehensive understanding of $\mathrm{Ni}-\mathrm{Cr}-\mathrm{Fe}-\mathrm{Nb}$ alloys, other composition variables are included to expand the model alloy systems. Ti is the strengthening element used in alloys 718 and 706 to promote the precipitation of $\gamma^{\prime}$. During solidification, it induces the formation of $\eta-\mathrm{Ni}_{3} \mathrm{Ti}$ in competition with the formation of Laves and $\delta-\mathrm{Ni}_{3} \mathrm{Nb}$. The interaction between $\mathrm{Ti}$ and $\mathrm{Nb}$ is studied by varying the $\mathrm{Ti} / \mathrm{Nb}$ ratio. The simulated results are validated by the observations in experimental $\mathrm{Ni}-\mathrm{Cr}-\mathrm{Fe}-\mathrm{Nb}-\mathrm{Ti}$ alloys. Low and high levels of Fe are also considered to simulate the chemistries of 718 and 706 type of alloys.

One of the important parameters for freckling study is the difference between liquidus and solidus, e.g. the freezing range. In a binary system, the freckling potential reaches the maximum value at the solubility limit where the freezing range becomes the largest. This represents the maximum contribution of temperature difference to the density change of liquid. The effect is difficult to visualize in multicomponent alloys due to the lack of phase diagrams. CALPHAD method is applied to calculate the solidification ranges in the above model alloys. The freezing ranges of experimental alloys are measured using DTA and the results are compared with prediction. Regression analysis is performed to generate relations between freezing range and key composition variables.

\subsubsection{CALPHAD Method in Solidification Simulation}

CALPHAD method provides a powerful tool for the understanding of materials behavior in equilibrium conditions. Under such conditions, critical temperatures, composition ranges for the formation of phases can be defined for multicomponent systems. However, traditional equilibria are not directly applicable to predicting the development of solidification structures, which is well away from equilibrium conditions. Simulation of 
solidification process is accomplished by coupling local equilibrium effect with global non-equilibrium assumptions.

Solidification under equilibrium conditions can be described by the lever rule and assuming linear liquidus and solidus lines, the composition of the solid (Cs) as a function of fraction solid transformed (fs) is given by the equation

$$
C_{S}=\frac{k C_{0}}{f_{S}(k-1)+1}
$$

where $\mathrm{k}$ is the partition coefficient and is constant during solidification, and $\mathrm{C}_{0}$ is the composition of the original liquid alloy. This can be re-arranged to give

$$
f_{S}=\frac{1}{(1-k)} \cdot\left(\frac{T_{L}-T}{T_{S}-T}\right)
$$

where $T_{L}$ and $T_{S}$ are the equilibrium liquidus and solidus temperatures. The limiting case to equilibrium solidification is to assume that solute diffusion in the solid phase is small enough to be considered negligible, and that diffusion in the liquid is extremely fast, fast enough to assume that diffusion is complete. The Scheil equation can be written as

$$
C_{S}=k C_{0}\left(1-f_{S}\right)^{k-1}
$$

and

$$
f_{S}=1-\left(\frac{T_{S}-T}{T_{S}-T_{L}}\right)
$$

The treatment above is the traditional derivation of the Scheil equation. However, it is not possible to derive the equation if the partition ratio, $k$, is dependent on temperature and/or composition, as in the case of multicomponent superalloys.

Using a special CALPHAD routine the disadvantage can be overcome. The physical process of Scheil solidification can be schematically shown in Figure 4-1. A liquid of composition $\mathrm{C}_{0}$ is cooled to a small amount below its liquidus to $\mathrm{T}_{1}$. It precipitates out solid with a composition $\mathrm{C}_{\mathrm{S} 1}$ and the liquid changes its composition to $\mathrm{C}_{\mathrm{L} 1}$. However, on further cooling to T2 the initial solid cannot change its composition owing to lack of diffusion and it is effectively 'isolated'. A local equilibrium is then set up where the liquid of composition $\mathrm{C}_{\mathrm{L} 1}$ transforms to a liquid $\mathrm{C}_{\mathrm{L} 2}$ and a solid with composition $\mathrm{C}_{\mathrm{S} 2}$ that is precipitated onto the original solid with composition $\mathrm{C}_{\mathrm{S} 1}$. This process occurs 
repeatedly during cooling and, when $\mathrm{k}<1$, leads to the solid being lean solute and the liquid becoming more and more enriched in solute as solidification proceeds. This procedure naturally treats the appearance of new phases at eutectic reactions or the disappearance of phases at peritectic reactions.

In the present study, equilibrium calculation module is used to obtain the phase diagrams. The Scheil module is applied to simulate the solidification of model alloys.

\subsection{Solidification Diagram of Ni-Cr-Fe-Nb System}

As-cast $\mathrm{Nb}$-containing $\mathrm{Ni}$-based superalloys consist of primary $\gamma$ phase, which is a facecentered-cubic solid solution of $\mathrm{Ni}, \mathrm{Cr}$, and $\mathrm{Fe}$, and secondary phases such as $\delta-\mathrm{Ni}_{3} \mathrm{Nb}$ and Laves. The segregation of $\mathrm{Nb}$ during solidification dominates the type of secondary phases to form. Quaternary $\mathrm{Ni}-\mathrm{Cr}-\mathrm{Fe}-\mathrm{Nb}$ system, which represents the simplified case of industrial alloys with $\gamma$ matrix and solute addition of $\mathrm{Nb}$, is of vital importance for the understanding of $\mathrm{Cr}, \mathrm{Fe}$, and $\mathrm{Nb}$ alloying effect. However, there is no detailed data available that can provide a comprehensive picture of the system. In this section, the advance CALPHAD approach will be used to develop the solidification diagram of the quaternary system. Four experimental Ni-Cr-Fe-Nb alloys, HV8853, CL-2, CL-3, CK-30 are selected to validate the theoretical prediction. The chemical compositions of model alloys are given in Table 2-1.

\subsubsection{Ternary Systems}

The liquidus surfaces of the two constituting ternary systems, $\mathrm{Ni}-\mathrm{Cr}-\mathrm{Nb}$ and $\mathrm{Ni}-\mathrm{Fe}-\mathrm{Nb}$, have been generated using Thermo-Calc and are shown in Figure 4-2 (a) and (b). The Ni$\mathrm{Cr}-\mathrm{Nb}$ diagram is identical to the diagram found in literature [45]. Primary Phase fields of $\gamma, \delta-\mathrm{Ni}_{3} \mathrm{Nb}$, and Laves, exist in both of the ternary solidification diagrams (Figure 4-2). Primary $\alpha-\mathrm{Cr}$ phase forms near the $\mathrm{Cr}$-rich corner. An invariant eutectic reaction of $\mathrm{L}$ $\rightarrow\left(\gamma+\delta-\mathrm{Ni}_{3} \mathrm{Nb}+\right.$ Laves) occurs in both systems (e2 and e3). Figure 4-2 (c) shows the projection of the univariant eutectic lines onto the $\mathrm{Nb}$ concentration versus temperature plane. Locations of e $2\left(1182^{\circ} \mathrm{C}\right)$ and e $3\left(1157^{\circ} \mathrm{C}\right)$ are the invariant eutectic points in the respective systems. Both $\mathrm{Cr}$ and $\mathrm{Fe}$ have partition ratios slightly higher than one based on 
the calculation, which means they are all matrix-forming elements and their partitioning behaviors are similar. Also the interactions of $\mathrm{Cr}-\mathrm{Nb}$ and $\mathrm{Fe}-\mathrm{Nb}$ are similar to each other according to the ternary liquidus projection. Due to the similarity, $\mathrm{Cr}$ and $\mathrm{Fe}$ can be treated as one single variable. Thus, it is expected that the Ni-rich $\mathrm{Ni}-\mathrm{Cr}-\mathrm{Fe}-\mathrm{Nb}$ system has a solidification surface similar to those of the ternaries if $[\mathrm{Cr}+\mathrm{Fe}]$ and $[\mathrm{Nb}]$ are used as the two variables. Formation of $\alpha-\mathrm{Cr}$ within the composition range of interest needs not be considered since the high $\mathrm{Cr}$ content is normally not seen in Ni-base superalloys.

\subsubsection{Quaternary System: CALPHAD Approach}

Scheil simulations were conducted on $\mathrm{Ni}-\mathrm{Cr}-\mathrm{Fe}-\mathrm{Nb}$ alloy compositions with systematic variation of $\mathrm{Cr}, \mathrm{Fe}$, and $\mathrm{Nb}$. (Composition ranges for calculation: $5<[\mathrm{Nb}]<20,5<[\mathrm{Cr}]$, $20<[\mathrm{Cr}+\mathrm{Fe}]<40$, all in wt pct). Most alloys encounter one of the two binary eutectics, $\gamma / \mathrm{Ni}_{3} \mathrm{Nb}$ and $\gamma /$ Laves, until the solidification is terminated by ternary $\mathrm{L} \rightarrow\left(\gamma+\mathrm{Ni}_{3} \mathrm{Nb}+\right.$ Laves) reaction. The calculated reaction sequences and eutectic amounts of the experimental alloys are given in Table 4-1.

Table 4-1: Calculated Solidification Sequences and Amounts of Eutectic

\begin{tabular}{ccccc}
\hline Alloy & Primary & Secondary Eutectic & Ternary Eutectic & Eutectic\% \\
\hline HV8853 & $\mathrm{L} \rightarrow(\mathrm{L}+\gamma)$ & $\mathrm{L} \rightarrow(\mathrm{L}+\gamma+\delta)$ & $\mathrm{L} \rightarrow(\gamma+\delta+$ Laves $)$ & 23 \\
CL-2 & $\mathrm{L} \rightarrow(\mathrm{L}+\gamma)$ & $\mathrm{L} \rightarrow(\mathrm{L}+\gamma+\delta)$ & $\mathrm{L} \rightarrow(\gamma+\delta+$ Laves $)$ & 67 \\
CL-3 & $\mathrm{L} \rightarrow(\mathrm{L}+\gamma)$ & $\mathrm{L} \rightarrow(\mathrm{L}+\gamma+\delta)$ & $\mathrm{L} \rightarrow(\gamma+\delta+$ Laves $)$ & 56 \\
CK-30 & $\mathrm{L} \rightarrow(\mathrm{L}+\gamma)$ & $\mathrm{L} \rightarrow(\mathrm{L}+\gamma+$ Laves $)$ & $\mathrm{L} \rightarrow(\gamma+\delta+$ Laves $)$ & 78 \\
\hline
\end{tabular}

Examples of temperature and liquid compositions versus fraction solid ( $f \mathrm{~s}$ ) of alloy HV8853 are shown in Figure 4-3. According to the prediction, these experimental alloys should form significant amounts of eutectic in solidification. Also binary and ternary eutectic constituents should be present in the interdendritic areas of as-cast alloys.

A pseudo-ternary solidification diagram of the $\mathrm{Ni}-\mathrm{Cr}-\mathrm{Fe}-\mathrm{Nb}$ system is shown in Figure 4-4. The calculated liquid $(\mathrm{Cr}+\mathrm{Fe})$ and $\mathrm{Nb}$ compositions of the experimental alloys were plotted on the solidification diagram. It was found that the compositions of the binary $\gamma / \mathrm{Ni}_{3} \mathrm{Nb}$ eutectic fall into the region bounded by e1, e2, and e3, and those of binary $\gamma /$ Laves locate in the region of e $2 \mathrm{e} 3 \mathrm{e} 4$. (The position of e 4 is arbitrary and it is not important in the 
current study since $\alpha-\mathrm{Cr}$ would be present in very small amount in the compositions considered). It is interesting that for most of the alloys calculated, the terminal eutectic compositions lie on a line connecting e 2 and e 3 , while e 2 and e 3 are the same as the ternary eutectic points in Figure 4-2. Obviously the monovariant lines of the ternary systems represent the extreme boundaries where $\mathrm{Cr}$ or $\mathrm{Fe}$ content reduces to 0 in the quaternary system.

\subsubsection{Experimental Results}

The average compositions of ternary $\left(\gamma+\mathrm{Ni}_{3} \mathrm{Nb}+\right.$ Laves $)$ eutectics in the experimental alloys were determined using EDS and given in Table 4-2. These measured eutectic compositions are distributed close to location e3 in Figure 4-4. According to Thermo-Calc prediction, these invariant compositions should distribute on a line connecting e 2 and e3. The deviation is caused by the difficulty in separating binary and ternary eutectics in EDS measurement since they are intermixed together.

Table 4-2: Average Eutectic Composition in Experimental Alloys by EDS (wt pct)

\begin{tabular}{cccccc}
\hline Alloy & $\mathrm{Ni}$ & $\mathrm{Cr}$ & $\mathrm{Fe}$ & $\mathrm{Cr}+\mathrm{Fe}$ & $\mathrm{Nb}$ \\
\hline HV8853 & 51.33 & 12.57 & 10.98 & 23.55 & 25.12 \\
CL-2 & 53.85 & 15.46 & 7.68 & 23.14 & 23.01 \\
CK-30 & 44.53 & 17.45 & 16.07 & 33.52 & 21.95 \\
CL-3 & 54.45 & 8.59 & 13.55 & 22.14 & 23.41 \\
\hline
\end{tabular}

The solidification sequence of a Ni-rich $\mathrm{Ni}-\mathrm{Cr}-\mathrm{Fe}-\mathrm{Nb}$ alloy predicted by the solidification diagram can be summarized as follows:

\begin{tabular}{llll}
\hline Primary & Secondary eutectic & & Terminal eutectic \\
\hline $\mathrm{L} \rightarrow(\mathrm{L}+\gamma) \Rightarrow$ & $\mathrm{L} \rightarrow(\mathrm{L}+\gamma+\delta) \quad$ or \\
& $\mathrm{L} \rightarrow(\mathrm{L}+\gamma+$ Laves $)$
\end{tabular}

Typical DTA solidification curves of alloy HV8853 are shown in Figure 4-5. A single eutectic reaction peak was detected at the cooling rate of $20^{\circ} \mathrm{C} / \mathrm{min}$ (curve $\mathrm{B}$ in Figure 4-5). Previous experience shows that slow cooling rates increase the sensitivity of the test [40]. Thus, a cooling rate of $5^{\circ} \mathrm{C} / \mathrm{min}$ was applied and the result is shown as curve $\mathrm{A}$ in Figure 45. Obviously two eutectic reactions occurred in the temperature range of eutectic reactions. 
The microstructure of DTA sample with $5^{\circ} \mathrm{C} / \mathrm{min}$ cooling exhibits two types of eutectic products, blocky and intermixed eutectic (Figure 4-6 (a)). The blocky-shaped phases were identified by EDS to have chemistry close to $\mathrm{Ni}_{3} \mathrm{Nb}$, however, it is difficult to discern the chemistry of individual phases in the intermixed eutectics due to the fine structure. It is believed to be the ternary eutectic of $\left(\gamma+\delta-\mathrm{Ni}_{3} \mathrm{Nb}+\right.$ Laves). A close-up of the intermixed eutectic is illustrated in Figure 4-6 (b). It is clear that the eutectic is an intimate mixture of phase with bright contrast, phase with less bright contrast, and the eutectic $\gamma$ (dark areas in between the bright phases). Similar eutectic morphologies were observed in CL-3. Qualitative EDS (Figure 4-7) of CL-3 shows that phase with bright contrast is enriched with $\mathrm{Nb}$ and $\mathrm{Ni}\left(\mathrm{Ni}_{3} \mathrm{Nb}\right)$, while phase with less bright contrast has high content of $\mathrm{Nb}$, equal amounts of $\mathrm{Ni}, \mathrm{Cr}$, and $\mathrm{Fe}$ (Laves). The result validates the prediction that HV8853 forms $\gamma / \delta-\mathrm{Ni}_{3} \mathrm{Nb}$ eutectic followed by the eutectic reaction of $\mathrm{L} \rightarrow(\gamma+\delta+$ Laves).

Reference [37] reported that alloy CK-30 forms Laves as the major eutectic constituent and no $\mathrm{Ni}_{3} \mathrm{Nb}$ was detected by X-Ray diffraction. This contradicts the prediction by Thermo-Calc that a ternary eutectic should form in the alloy. The microstructure of the alloy exhibits a large amount of $\gamma /$ Laves eutectic (Figure 4-8 (a)). The close-up of the eutectic region shows that the Laves lamella are coated with a layer of phase with brighter contrast in the BSE image (Figure 4-8 (b)). Trace amounts of small brighter particles are also observed besides the Laves eutectic. It is difficult to determine the chemistry of these bright phases due to the size limit in EDS. From the morphology, they are formed at the very last stage of solidification. This indicates that alloy CK-30 does form ternary eutectic at the final stage of solidification although the amount of $\mathrm{Ni}_{3} \mathrm{Nb}$ is too small to be detected by X-Ray diffraction. The intermixed ternary eutectic morphologies are also observed in CL-2 and CL-3, as shown in Figure 4-9.

\subsubsection{Alloying Effects}

In order to form binary $\gamma /$ Laves eutectic in the $\mathrm{Ni}-\mathrm{Cr}-\mathrm{Fe}-\mathrm{Nb}$ system, the combined $[\mathrm{Cr}+\mathrm{Fe}]$ content must exceed a certain value, which lies between 32 wt pct and 35 wt pct. This is consistent with previous prediction [37]. Since HV8853 represents the case in which only ternary eutectic could form under fast cooling conditions, the relationship of its $[\mathrm{Cr}+\mathrm{Fe}]$ 
and $\mathrm{Nb}$ liquid concentrations can be used as an estimate to predict what binary eutectic will form in an alloy. In Figure $4-10, \mathrm{AB}$ is defined as $[\mathrm{Cr}]+[\mathrm{Fe}]+0.9[\mathrm{Nb}]=44$. If an alloy has a composition located above $\mathrm{AB}([\mathrm{Cr}]+[\mathrm{Fe}]+0.9[\mathrm{Nb}]>44)$ in the primary $\gamma$ phase field, it will form $\gamma /$ Laves eutectic. If the composition is located below $A B, \gamma / \mathrm{Ni}_{3} \mathrm{Nb}$ eutectic results. In both cases, solidification terminates with invariant $\mathrm{L} \rightarrow(\gamma+\delta+$ Laves $)$ reaction.

When Fe content is increased, the two-fold $\gamma /$ Laves and $\gamma / \delta$ univariant lines are shifted towards the direction of higher $\mathrm{Nb}$. The solidification range is enlarged assuming constant $\mathrm{Nb}$ solubility. This reduces the total amount of eutectic in the alloy. Hence, the amount of Laves is reduced. This is consistent with the discovery [37] that Cr has a more pronounced effect to form more Laves eutectic than Fe. The trend is show in Figure 4-10.

The pseudo-solidification diagram of the $\mathrm{Ni}-\mathrm{Cr}-\mathrm{Fe}-\mathrm{Nb}$ system generally describes the solidification behavior of $\mathrm{Nb}$-containing superalloys consisting of $\gamma$ matrix with $\mathrm{Ni}, \mathrm{Cr}$, and $\mathrm{Fe}$ as the major constituents and Nb-rich secondary phases, such as Laves and $\mathrm{Ni}_{3} \mathrm{Nb}_{\text {. }}$ Most of the commercial Ni-base Nb-containing superalloys have basic alloying chemistries similar to the quaternary alloys in the present study. However, caution is advised to apply the current diagram to commercial alloys due to the presence of other solute elements such as $\mathrm{Mo}, \mathrm{Ti}, \mathrm{Si}$, and $\mathrm{C}$. These alloying additions will influence the solidification behavior as well as the types of secondary phases to form. Current version of $\mathrm{NiFe}$-database does not contain $\mathrm{C}$ and $\mathrm{Si}$. Future work is needed to incorporate these alloying effects.

\subsection{Freezing Range of Model Alloys}

CALPHAD prediction yields two important outputs, i.e. fraction of solid and freezing range, for freckling study in superalloys. For binary alloys, these data can readily be obtained from available phase diagrams. However, there is no phase diagram for superalloys due to the complex chemistry. The difficulty can now be avoided by using CALPHAD method which offers the advantage of theoretically predicting these parameters through thermodynamic computation.

In this section, alloying effects on the freezing ranges of $\mathrm{Ni}-\mathrm{Cr}-\mathrm{Fe}-\mathrm{Nb}-\mathrm{Ti}$ and $\mathrm{Ni}-$ $\mathrm{Cr}-\mathrm{Fe}-\mathrm{Nb}-\mathrm{Ti}-\mathrm{Al}$ model alloys are thoroughly investigated. Predicted results are compared with experimental data from DTA for validation. Fractions of solid versus temperature 
relations are calculated by Thermo-Calc using the alloy compositions given in Table 2-1. The predicted results, together with experimental determination of fraction solidified versus temperature curves will be described in detail in the chapter 6 .

\subsubsection{Freezing Range ( $\left.\Delta T=T_{L}-T_{S}\right)$}

Liquidus $\left(T_{L}\right)$ of an alloy is defined as the temperature at which solidification begins. The definition of solidus $\left(T_{S}\right)$ varies from study to study. In binary phase diagram, solidus temperature is the end of equilibrium solidification. However, real solidification occurs in non-equilibrium manner and extends into eutectic region. In this case, solidus is the temperature at which the primary solidification ends and eutectic reaction begins. In Thermo-Calc, the solidus can be detected as the first deflection point on the $f \mathrm{~s}(T)$ curve during solidification. In DTA, it is the end point of the primary solidification curve. The last two definitions are used in the current study.

Freezing range data are extracted from the Thermo-Calc simulations of model alloys. The results are given in Table 4-3. Experimental results are obtained from DTA tests with a constant cooling rate of $20^{\circ} \mathrm{C} / \mathrm{min}$ (Table 4-3). The temperatures at the end of eutectic solidification, $T_{E}$, are also included in Table 4-3. The experimental values of freezing range are compared with those predicted by CALPHAD, as shown in Figure 4-11. The comparison exhibits fair correlation between the experimental and theoretical values. The correlation between experimental and predicted values are given as:

$$
\Delta T_{T C}=1.299 \cdot \Delta T_{D T A}-40.7876, R_{a d j}^{2}=0.855 .
$$

The significant correlation shows that Thermo-Calc is capable to predict the trend of freezing ranges among alloys. For example, Thermo-Calc shows that HV8853 has a larger freezing range than HV8852, which agrees with DTA findings. However, difference of absolute values between the two methods has been observed. The discrepancy can be attributed to the DTA equipment error, alloy composition, and kinetic effect that is not considered in Thermo-Calc. As stated in chapter 2, the equipment error of DTA is calibrated to be within $\pm 2{ }^{\circ} \mathrm{C}$. The kinetic effect is assumed to be small under the current DTA experimental conditions, future work is needed to quantify this effect. In order to illustrate the alloying effect on the discrepancy, correlation coefficients between the 
characteristic temperature difference and compositions using Microsoft Excel were shown in Table 4-4.

Table 4-4: Correlation of difference in characteristic temperature (between DTA and Thermo-Calc) and alloy compositions.

\begin{tabular}{cccc}
\hline Composition & $\Delta T_{\mathrm{DTA}}-\Delta T_{\mathrm{TC}}$ & $T_{\mathrm{S}, \mathrm{DTA}}-T_{\mathrm{S}, \mathrm{TC}}$ & $T_{\mathrm{L}, \mathrm{DTA}}-T_{\mathrm{L}, \mathrm{TC}}$ \\
\hline $\mathrm{Cr}$ & 0.21 & -0.18 & 0.16 \\
$\mathrm{Fe}$ & 0.39 & -0.14 & 0.47 \\
$\mathrm{Nb}$ & 0.31 & -0.51 & 0.01 \\
$\mathrm{Ti}$ & 0.33 & -0.16 & 0.37 \\
$\mathrm{C}$ & 0.30 & -0.43 & 0.06 \\
$\mathrm{Si}$ & 0.62 & -0.55 & 0.45 \\
$\mathrm{Al}$ & -0.34 & 0.16 & -0.37 \\
\hline
\end{tabular}

A correlation coefficient of 1 means strong positive correlation between the two sets of data and vice versa, while 0 means no correlation. Si has significant effect in enlarging the freezing range in DTA as compared with Thermo-Calc. This is realized by increasing liquidus and reducing solidus temperatures in DTA. It is because $\mathrm{Si}$ is not included in $\mathrm{NiFe}$-database for calculation. Fe tends to increase the liquidus and $\mathrm{Nb}$ tends to decrease the solidus in DTA measurement as compared with Thermo-Calc. The three 706-type alloys have the noticeable difference between Thermo-Calc and DTA. It is postulated that the coexistence of high contents of $\mathrm{Nb}, \mathrm{Ti}$, and $\mathrm{Fe}$ may cause the deviation. More study is needed to substantiate the composition effect. It is cautioned that other factors must be considered together with composition effect to explain the discrepancy between ThermoCalc and experimental methods.

No correlation can be found between total solidification range $\left(T_{L}-T_{E}\right)$ obtained from DTA and Thermo-Calc prediction. The discrepancy is attributed to the predictability at the end of solidification by Thermo-Calc, where the composition of the solute-rich liquid is much higher than nominal composition. Thermo-Calc and the database are not intended for the use at these enriched concentrations.

\subsubsection{Alloying Effects}

Multi-linear regression analysis is conducted to correlate the freezing range with major alloy compositions. The equation for those obtained using Thermo-Calc is 


$$
\Delta T_{T C}=724.9-19.225 \cdot[\mathrm{Cr}]-3.279 \cdot[\mathrm{Fe}]-18.434 \cdot[\mathrm{Nb}]-9.228 \cdot[\mathrm{Ti}]
$$

The adjusted $\mathrm{R}$ square value represents the significance of regression, $R_{a d j}^{2}=0.925$. Terms in brackets are the weight percentage concentrations. For the freezing ranges by DTA, the regression equation is

$$
\Delta T_{D T A}=463.867-10.2576 \cdot[\mathrm{Cr}]-1.491 \cdot[\mathrm{Fe}]-12.77 \cdot[\mathrm{Nb}]-5.297 \cdot[\mathrm{Ti}]
$$

$R_{a d j}^{2}=0.88$. Both of the regression analysis are statistically significant. Equations (4-6) and (4-7) can be applied to the following composition ranges: $16<[\mathrm{Cr}]<22,4<[\mathrm{Fe}]<36$, $3<[\mathrm{Nb}]<14,0<[\mathrm{Ti}]<5.6$, all in wt pct. Comparisons of regressions are shown in Figure 412 (a) and (b). It is interesting to note that the order of alloying effects predicted by Thermo-Calc is similar to that obtained from DTA, which again indicates good predictability of Thermo-Calc.

Results show that all the alloying additions considered decrease the freezing range to some extent. Among them, $\mathrm{Nb}$ and $\mathrm{Cr}$ have the most significant effects. This is because the most of the model alloys have solute concentrations higher than their solubilities in matrix. According to the binary phase diagram as shown in Figure 4-1, the increase of solute concentration tends to decrease freezing range once it exceeds the solubility. However, if it is smaller than the solubility, the increase of the solute concentration will increase the freezing range. This effect restricts the application of equations (4-6) and (47) for dilute alloys. More work is needed to develop alloying effect on freezing range of dilute alloys.

\subsection{Solidification Sequence and Secondary Phases}

\subsubsection{Solidification Sequence}

The prediction of secondary phases in experimental alloys can be illustrated in Figure 413. The size, shape and intermixed morphologies of the phases can be used to determine their reaction sequences. For example, two stages of eutectic reactions happen in HV8582 (Figure 4-13 (a)). The large kidney-shaped phases are $\delta-\mathrm{Ni}_{3} \mathrm{Nb}$, which is formed in the binary eutectic reaction right after the primary solidification of $\gamma$ phase. During the eutectic reaction, $\gamma$ phase continues to grow on the primary dendrites, while $\delta$ islands form 
in the interdendritic liquid. As the temperature continues to decrease, a ternary eutectic reaction occurs when the residual liquid transforms into $\gamma+\delta+$ Laves intermixed eutectics at the end of solidification. These eutectic constituents are adjacent to the kidney-shaped phases. Micro-porosity (less than 1 micron) near the ternary eutectic indicates that shrinkage occurs during the last stage of solidification. The argument is substantiated by the DTA cooling curve (Figure 4-13 (b)) in which two distinctive reaction peaks are detected during the last stage of solidification.

\subsubsection{Secondary Phases}

Three types of secondary phases, i.e. Laves, $\delta-\mathrm{Ni}_{3} \mathrm{Nb}$, and $\eta-\mathrm{Ni}{ }_{3} \mathrm{Ti}$, form in eutectic reactions of $\mathrm{Ni}-\mathrm{Cr}-\mathrm{Fe}-\mathrm{Nb}-\mathrm{Ti}-(\mathrm{Al})$ alloys. It was shown [12] that the amount of $\eta$ phase increases with the $\mathrm{Ti} / \mathrm{Nb}$ ratio, while the amount of Laves decreases at the same time. $\mathrm{Ti}$ seems to have a significant effect on the formation of secondary phases during eutectic solidification in a way that only trace amount of $\delta$ phase is seen in Thermo-Calc prediction and none has been observed in experimental alloys. Thus, CALPHAD method can be used to investigate the competition effects between $\mathrm{Ti}$ and $\mathrm{Nb}$ quantitatively.

A series of Ni-Cr-Fe-Nb-Ti alloy compositions are selected for Scheil simulation. $\mathrm{Cr}$ and $\mathrm{Fe}$ contents are kept at 20 and 18 wt pct respectively, two levels of $\mathrm{Ti}$ and $\mathrm{Nb}$ additions are used as shown in Table 4-5. The compositions of experimental alloys are also used in the calculation. Solidification sequences and volume fractions of secondary phases are extracted from the data files. It is shown that $\delta$ phase only appears in alloys with small $\mathrm{Ti} / \mathrm{Nb}$ ratio. With the increase of $\mathrm{Ti} / \mathrm{Nb}$ ratio, Laves and $\eta$ phases become the major eutectic constituents. If the ratio exceeds certain limits, $\eta$ phase reaction becomes the first eutectic reaction in sequence.

In 706-type of model alloys (HV8523, HV8524, and HV8552), only Laves phase is seen in the interdendritic region. These alloys have $36 \mathrm{wt}$ pct Fe and significant amounts of $\mathrm{Nb}$ and $\mathrm{Ti}$. It is believed that a single eutectic reaction of Laves/ $\gamma$ happens in these alloys. (Figure 4-14)

In 718-type of model alloys (HV8551 and HV8550, with 1 wt pct Al), two types of globular Laves particles form an intermixed structure with $\eta$ phase (gray contrast), as shown in Figure 4-15 (a) and (b). The difference of particle size is attributed to the 
difference of their reaction temperatures. The finer Laves particles are believed to form at a lower temperature than that of bigger particles during solidification. However, the two reaction temperatures should be very close due to the intimate structure and a single eutectic reaction on DTA curve of the alloy (Figure 4-15 (c)). DTA may not be sensitive enough to pick up the two reactions that are very close in their reaction temperatures.

\subsubsection{Composition Effects}

Excessive secondary eutectic phases in superalloys can cause incipient melting problem in high temperature forging and welding processes. However, no data is available in literature to illustrate the correlation between amount of secondary phase and composition variables. In this study, Thermo-Calc is used to calculate the amounts of secondary phases in model alloy systems. Regression analysis is attempted to determine the relationship between the amount of Laves and alloying additions. The result is given as follows

$$
\text { Laves }(\%)=-60.6481+1.695[\mathrm{Cr}]+0.738[\mathrm{Fe}]+2.799[\mathrm{Nb}]+1.208[\mathrm{Ti}], \quad R_{a d j}^{2}=0.934
$$

It is seen that increase of alloying contents will increase the amount of Laves formed in the alloy, with the effects diminishing in the order of $\mathrm{Nb}, \mathrm{Cr}, \mathrm{Ti}$, and $\mathrm{Fe}$. The result is consistent with the prediction from the solidification diagram of $\mathrm{Ni}-\mathrm{Cr}-\mathrm{Fe}-\mathrm{Nb}$, which indicates that $\mathrm{Cr}$ and $\mathrm{Nb}$ have more pronounced effects on the formation of Laves. Fe has been repeatedly shown to be responsible for Laves formation in Ni-based superalloys. However, equation (4-8) and the solidification diagram of $\mathrm{Ni}-\mathrm{Cr}-\mathrm{Fe}-\mathrm{Nb}$ show that the effect of $\mathrm{Cr}$ addition is more significant than $\mathrm{Fe}$. No $\mathrm{Al}$ effect on Laves formation can be found by the regression analysis.

The regression relationship between the amount of $\eta$ phase and alloying additions is given by the following equation

$$
\operatorname{Eta}(\%)=38.34-1.7267[\mathrm{Cr}]-0.522[\mathrm{Fe}]+4.3171[\mathrm{Ti}]-4.81[\mathrm{Al}], \quad R_{a d j}^{2}=0.959
$$

The increase of Ti content will increase the amount of $\eta$ phase formed in the alloy. This is in good agreement with experimental observation. However, other alloying additions such as $\mathrm{Cr}, \mathrm{Fe}$, and $\mathrm{Al}$, tend to reduce the amount of $\eta$.

Both equations (4-8) and (4-9) can be applied to alloys with the following composition ranges: $[\mathrm{Cr}]=20,[\mathrm{Fe}]=18$ or $36,4<[\mathrm{Nb}]<12,1<[\mathrm{Ti}]<9,0<[\mathrm{Al}]<1$, in 
wt pct. Again the direct application of equations (4-8) and (4-9) is cautioned since $\mathrm{Nb}$ and Ti levels in most of the compositions are equal to or higher than solid solubilities.

\subsection{Summary}

Results and discussions in chapter 4 are summarized as follows:

1. Model superalloys are designed and systematically investigated using CALPHAD.

2. Solidification behaviors, such as solidification sequence, the amount and occurrence of secondary phases, and freezing ranges of model alloys, can be simulated using CALPHAD method with relatively good predictability.

3. A pseudo-solidification diagram of $\mathrm{Ni}-\mathrm{Cr}-\mathrm{Fe}-\mathrm{Nb}$ alloy system by using $[\mathrm{Cr}+\mathrm{Fe}\}$ and $\mathrm{Nb}$ as the two variables. The predicted results exhibit good consistency with actual data of the experimental alloys, which demonstrated the merits of the method.

4. Cr has some more pronounced effect than Fe in promoting Laves formation.

5. $\mathrm{Cr}$ and $\mathrm{Nb}$ have more significant effects in reducing the freezing range than $\mathrm{Fe}$ and $\mathrm{Ti}$. Equations (4-6) and (4-7) are restricted for alloys with high levels of $\mathrm{Nb}$ and $\mathrm{Ti}$. Application to dilute alloys is restricted.

6. $\mathrm{Nb}$ have more pronounced effect on Laves formation that other elements as shown in equation (4-8). Ti increases the amount of $\eta$ phase, while $\mathrm{Cr}, \mathrm{Fe}$, and $\mathrm{Al}$ all suppress the formation of $\eta$, as shown in equation (4-9). Again both equation are restricted for alloys with high levels of $\mathrm{Nb}$ and Ti. Application to dilute alloys is restricted. 


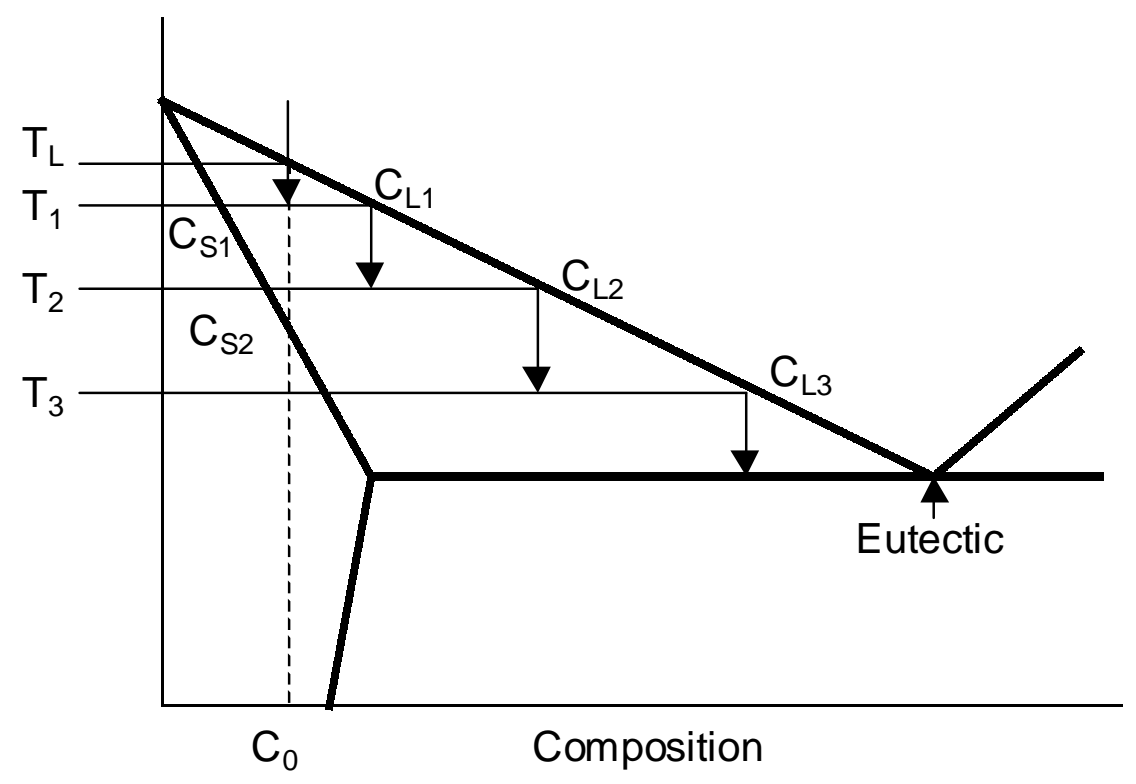

Figure 4-1: Schematic of Scheil solidification module in Thermo-Calc.

Table 4-3: Freezing range $\left(\mathrm{T}_{\mathrm{L}}-\mathrm{T}_{\mathrm{S}}\right)$ obtained using Thermo-Calc and DTA $\left({ }^{\circ} \mathrm{C}\right)$

\begin{tabular}{c|ccc|ccc|cc|cc}
\hline \multirow{2}{*}{ Alloy } & \multicolumn{3}{|c|}{ DTA } & \multicolumn{3}{c|}{ Thermo-Calc } & \multicolumn{2}{c|}{ DTA } & \multicolumn{2}{c}{ Thermo-Calc } \\
\cline { 2 - 11 } & $\mathrm{T}_{\mathrm{L}}$ & $\mathrm{T}_{\mathrm{S}}$ & $\mathrm{T}_{\mathrm{E}}$ & $\mathrm{T}_{\mathrm{L}}$ & $\mathrm{T}_{\mathrm{S}}$ & $\mathrm{T}_{\mathrm{E}}$ & $\mathrm{T}_{\mathrm{L}}-\mathrm{T}_{\mathrm{S}}$ & $\mathrm{T}_{\mathrm{L}}-\mathrm{T}_{\mathrm{E}}$ & $\mathrm{T}_{\mathrm{L}}-\mathrm{T}_{\mathrm{S}}$ & $\mathrm{T}_{\mathrm{L}}-\mathrm{T}_{\mathrm{E}}$ \\
\hline HV8851 & 1348 & 1264 & 1239 & 1345 & 1250 & 1222 & 84 & 109 & 95 & 123 \\
HV8852 & 1356 & 1242 & 1196 & 1340 & 1228 & 1189 & 114 & 160 & 112 & 151 \\
HV8853 & 1356 & 1176 & 1144 & 1369 & 1190 & 1183 & 180 & 213 & 179 & 186 \\
HV8549 & 1292 & 1146 & 1103 & 1281 & 1161 & 1140 & 146 & 188 & 121 & 141 \\
HV8520 & 1274 & 1155 & 1100 & 1264 & 1145 & 1134 & 119 & 173 & 119 & 130 \\
HV8524 & 1307 & 1192 & 1092 & 1254 & 1195 & 1151 & 115 & 215 & 58 & 103 \\
HV8523 & 1307 & 1201 & 1104 & 1276 & 1202 & 1153 & 106 & 203 & 74 & 123 \\
HV8552 & 1279 & 1212 & 1109 & 1255 & 1212 & 1153 & 68 & 171 & 43 & 102 \\
HV8551 & 1280 & 1141 & 1091 & 1269 & 1135 & 1089 & 139 & 189 & 134 & 180 \\
HV8521 & 1290 & 1147 & 1097 & 1294 & 1135 & 1098 & 143 & 194 & 159 & 196 \\
HV8522 & 1242 & 1147 & 1089 & 1244 & 1147 & 1089 & 94 & 153 & 97 & 155 \\
HV8550 & 1274 & 1158 & 1097 & 1268 & 1152 & 1099 & 116 & 176 & 116 & 169 \\
HV8582 & 1279 & 1198 & 1131 & 1271 & 1209 & 1185 & 81 & 148 & 62 & 86 \\
HV8583 & 1244 & 1176 & 1129 & 1239 & 1184 & 1147 & 68 & 115 & 55 & 92 \\
718 & 1342 & 1175 & 1131 & 1353 & 1140 & 1117 & 167 & 211 & 212 & 236 \\
706 & 1393 & 1189 & 1153 & 1391 & 1170 & 1132 & 204 & 240 & 221 & 259 \\
625 & 1353 & 1177 & 1138 & 1350 & 1145 & 1110 & 176 & 215 & 205 & 240 \\
\hline
\end{tabular}

Note: $T_{L}$ is the liquidus, $T_{S}$ is the solidus, $T_{E}$ is the temperature at the end of eutectic reaction. 


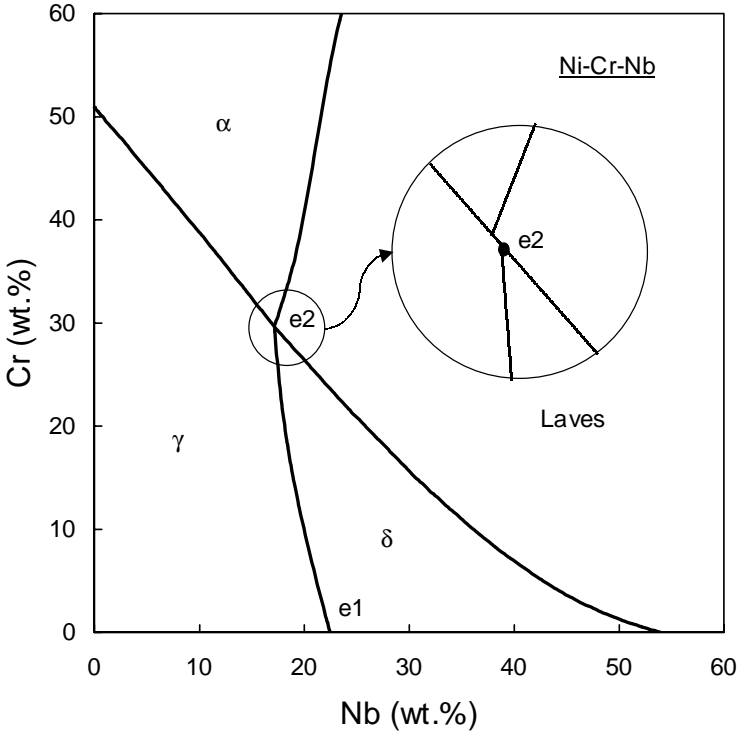

(a)

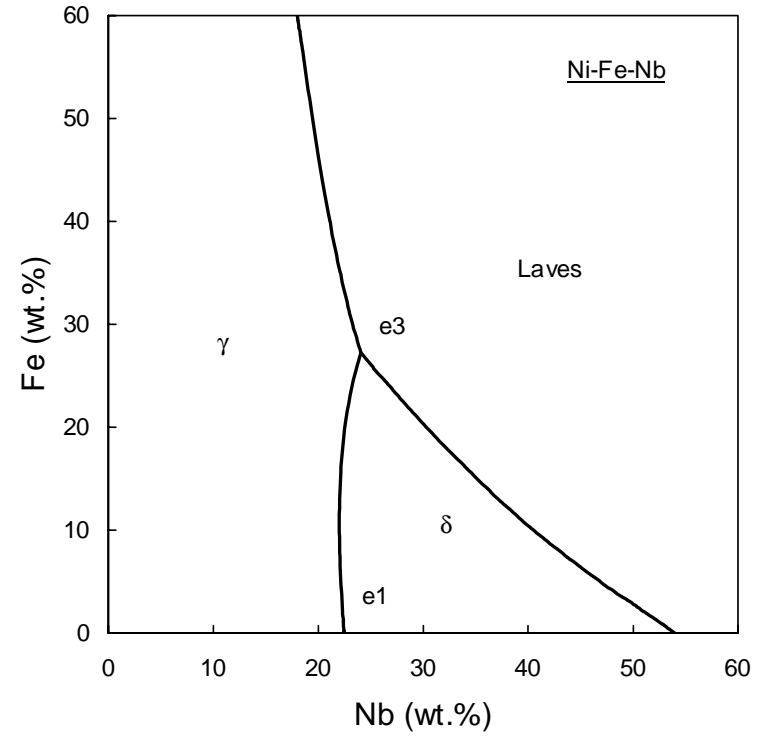

(b)

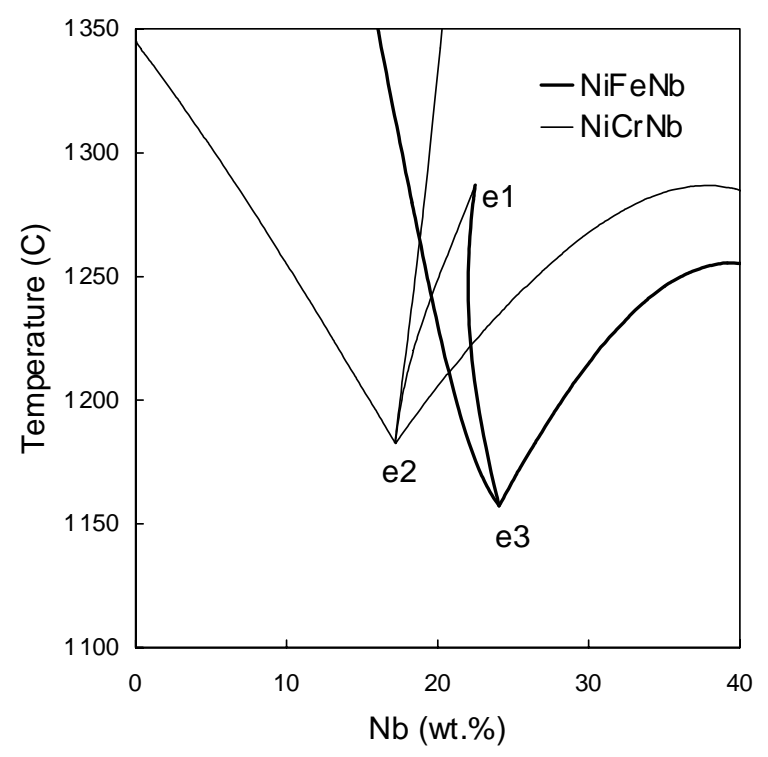

(c)

Figure 4-2: Liquidus projection diagrams of (a) $\mathrm{Ni}-\mathrm{Cr}-\mathrm{Nb}$ and (b) $\mathrm{Ni}-\mathrm{Fe}-\mathrm{Nb}$ systems calculated using Thermo-Calc. The projection of monovariant eutectic lines onto the $\mathrm{Nb}$ vs. temperature plane of both systems is given in (c). 


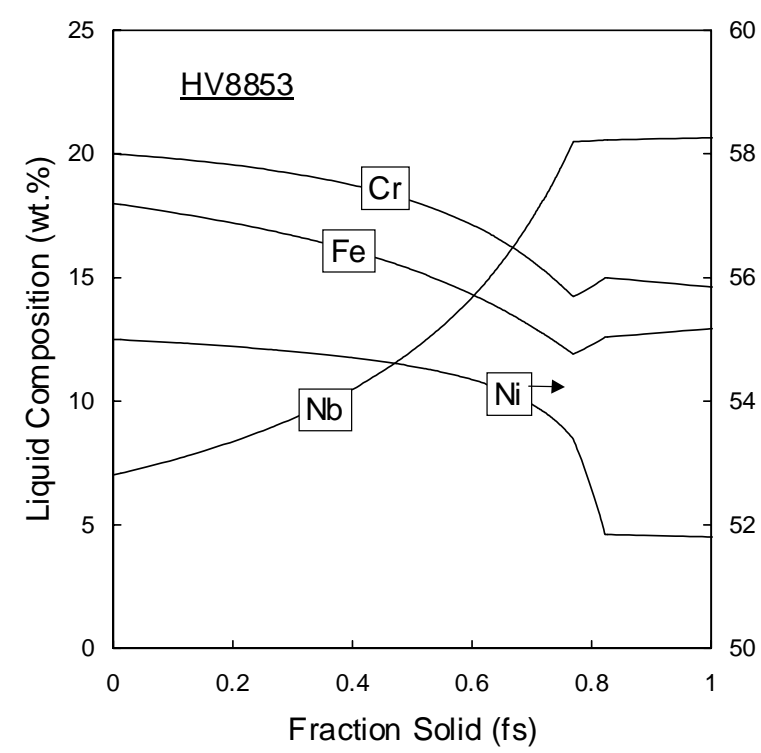

(a)

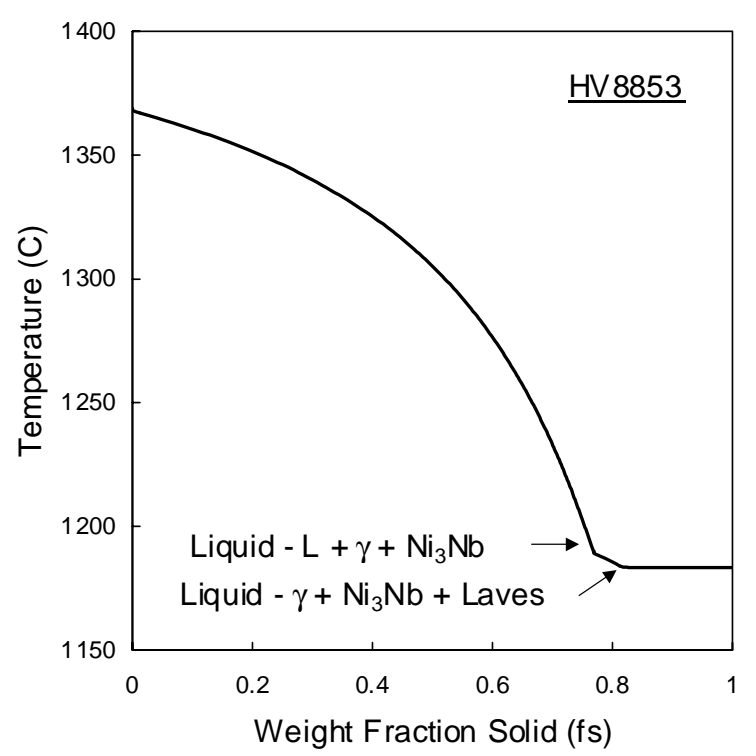

(b)

Figure 4-3: Scheil solidification simulation of HV8853, (a): fraction solid vs. temperature and (b): liquid composition vs. fraction solid.

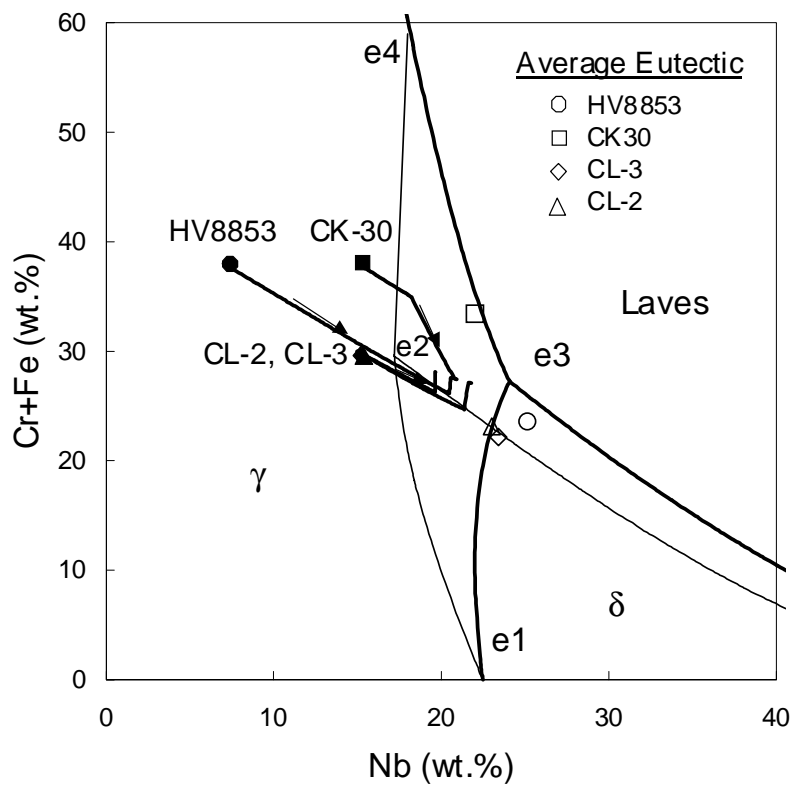

Figure 4-4: Pseudo-ternary solidification diagram of Ni-Cr-Fe-Nb alloy system. 


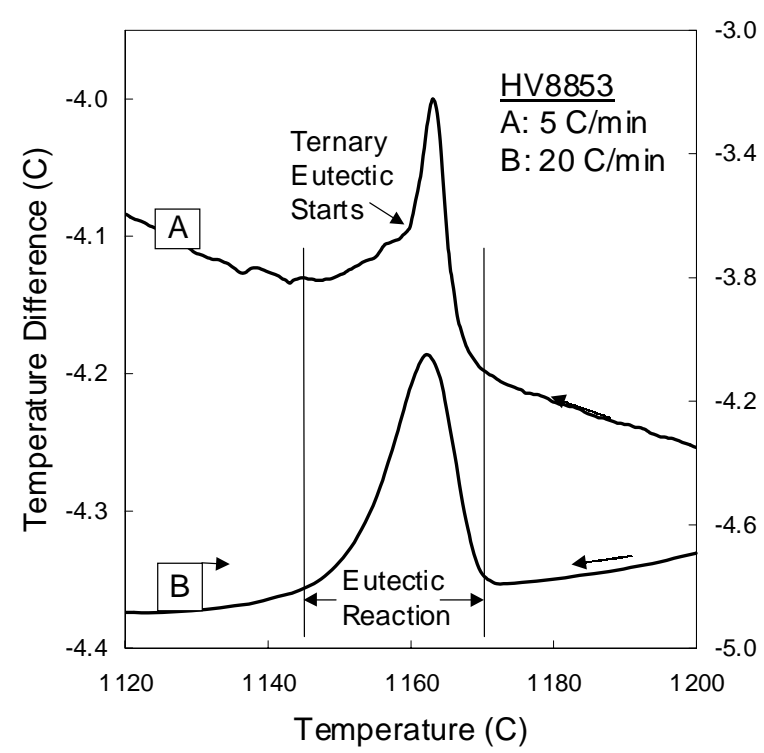

Figure 4-5: DTA cooling curve of HV8853 at 20 and $5{ }^{\circ} \mathrm{C} / \mathrm{min}$ showing eutectic reactions.

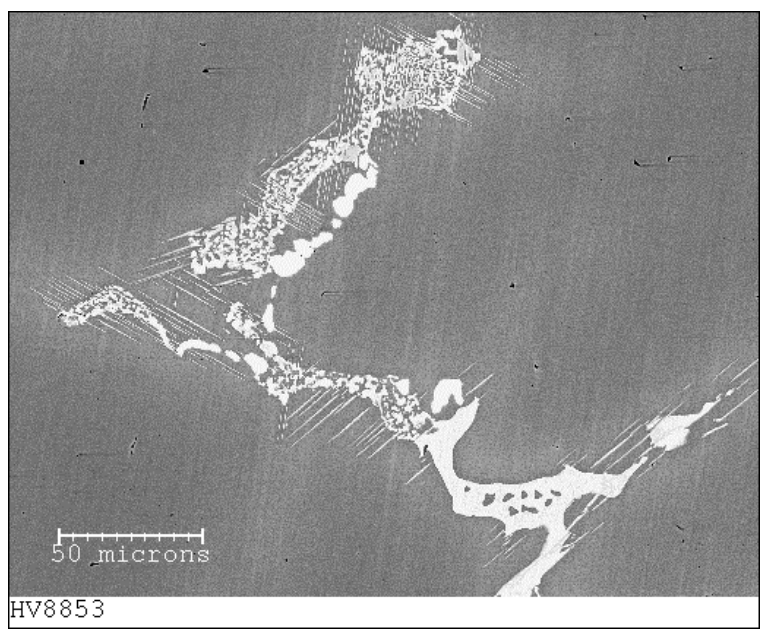

(a)

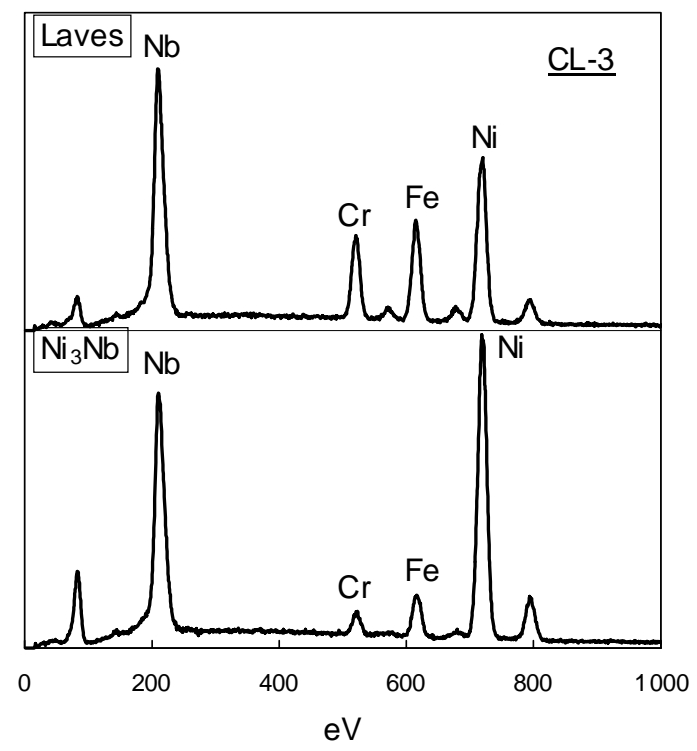

Figure 4-7: EDS spectrums of eutectic phases in alloy CL-3.

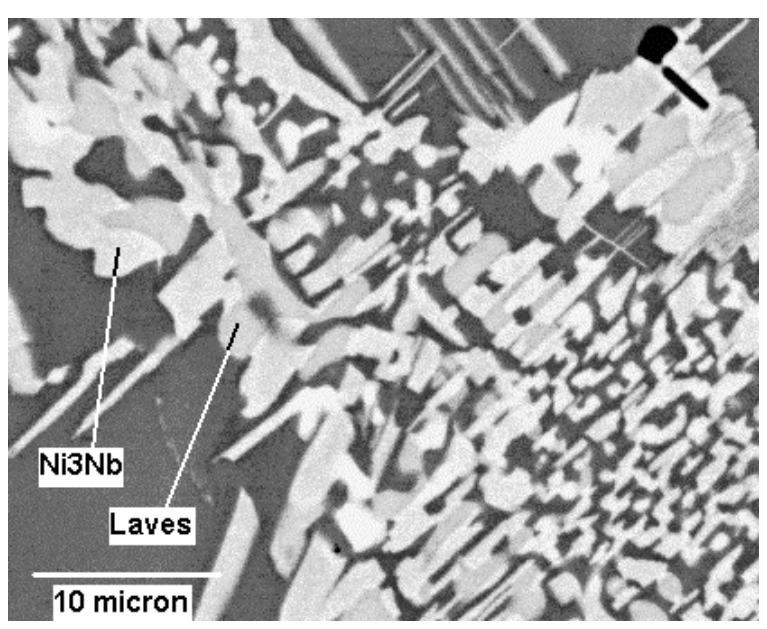

(b)

Figure 4-6: Back-scattered electron images of HV8853 (DTA, $5^{\circ} \mathrm{C} / \mathrm{min}$ ). 

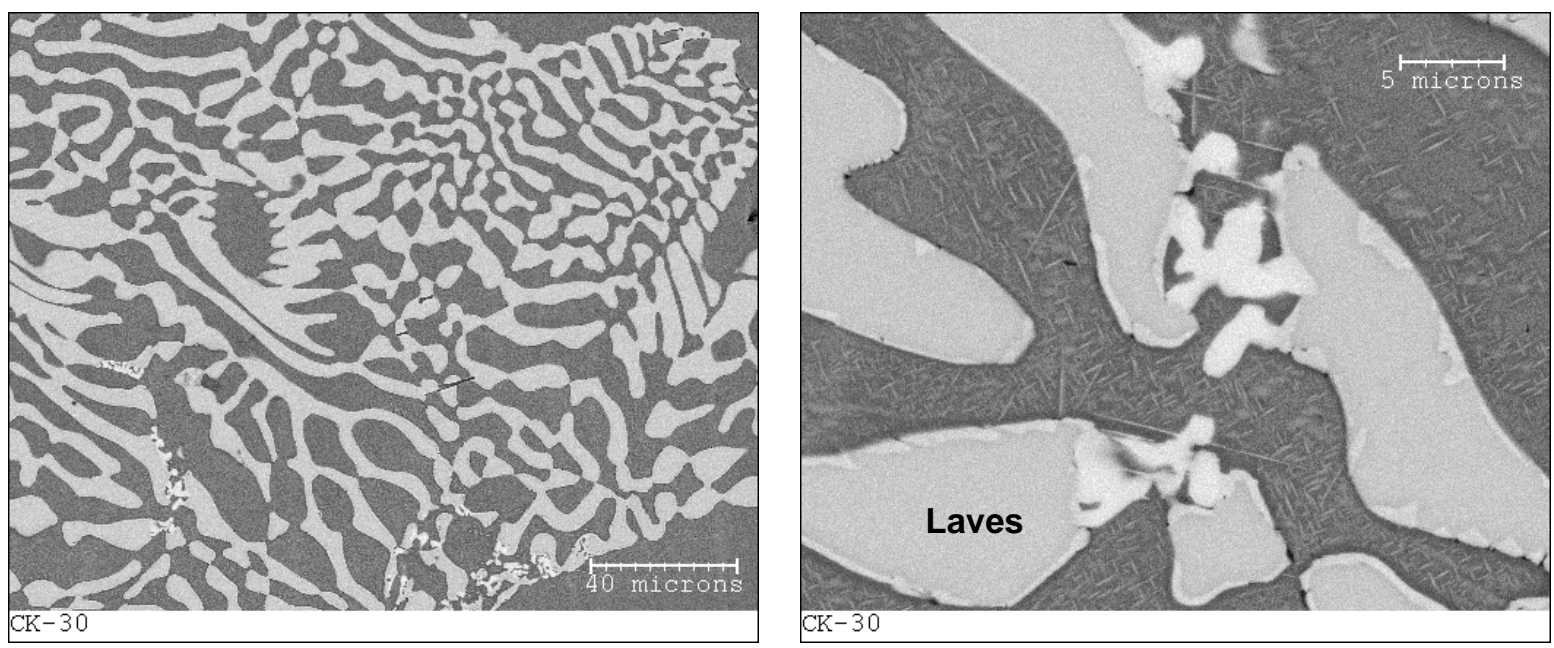

Figure 4-8: Back-scattered electron images of as-cast CK-30.

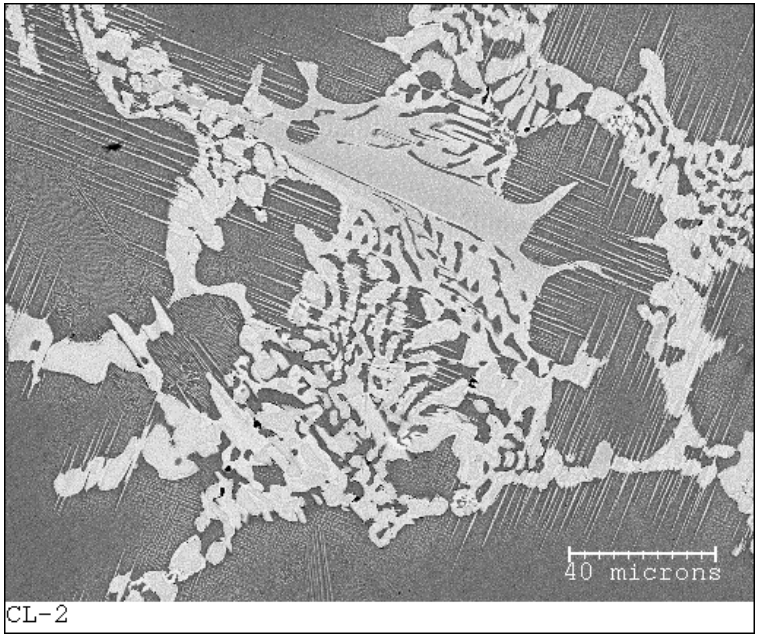

(a)

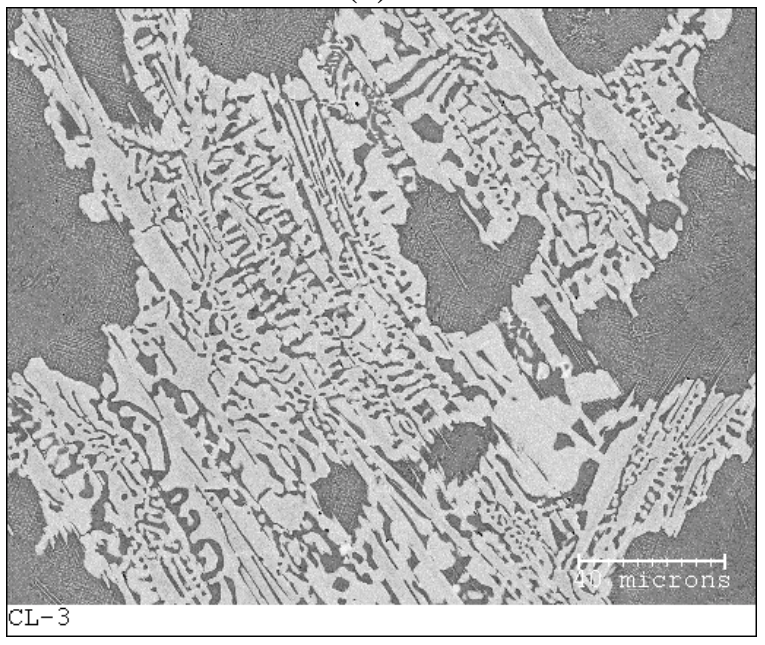

(c)

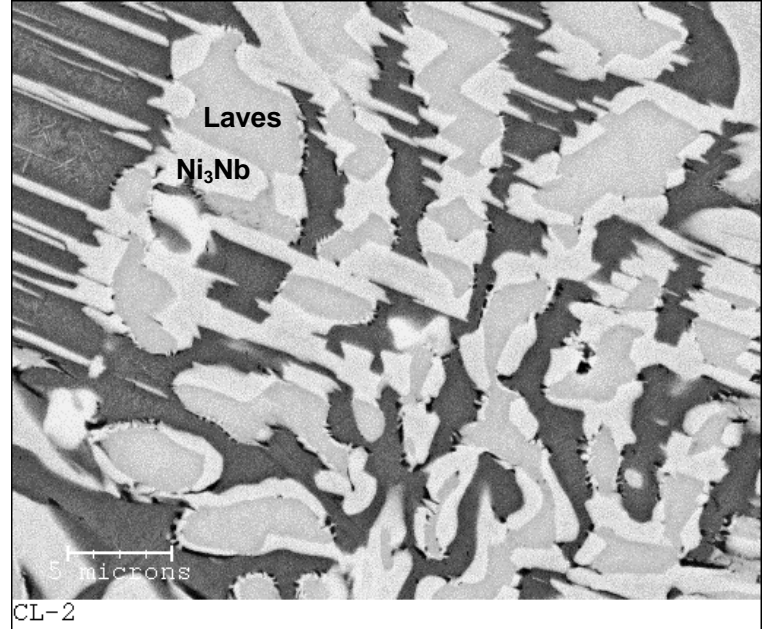

(b)

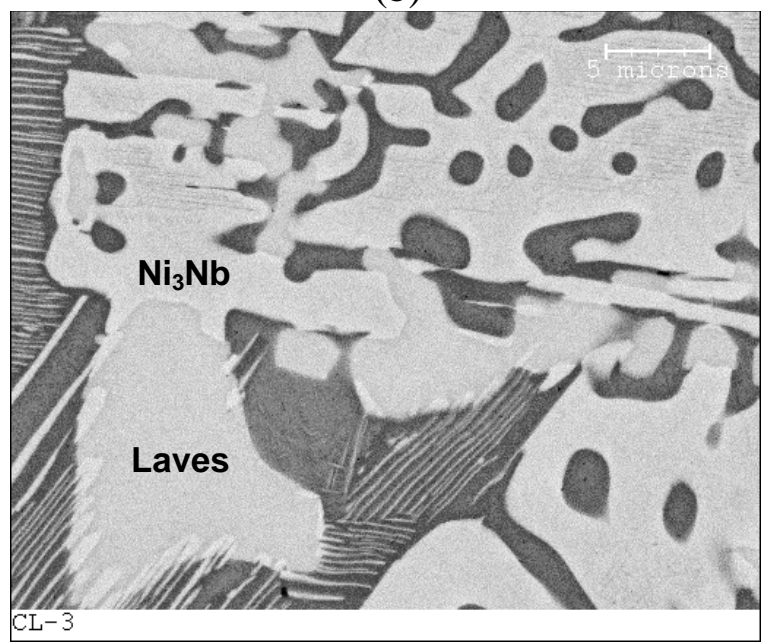

(d)

Figure 4-9: BSE images of CL-2 where the bright phases on the edge are $\mathrm{Ni}_{3} \mathrm{Nb}$ (a) and (b). BSE images of CL-3 where the bright phases are $\mathrm{Ni}_{3} \mathrm{Nb}$ (c) and (d). 


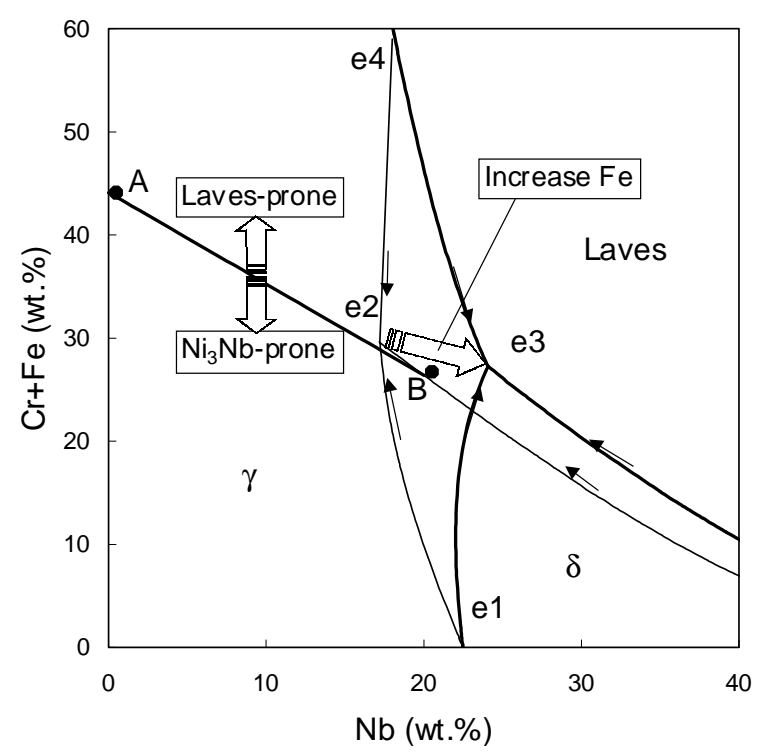

Figure 4-10: Solidification diagram of Ni-Cr$\mathrm{Fe}-\mathrm{Nb}$ showing alloying effects on formation of secondary phases. $\mathrm{AB}:[\mathrm{Cr}]+[\mathrm{Fe}]+0.9[\mathrm{Nb}]=44$

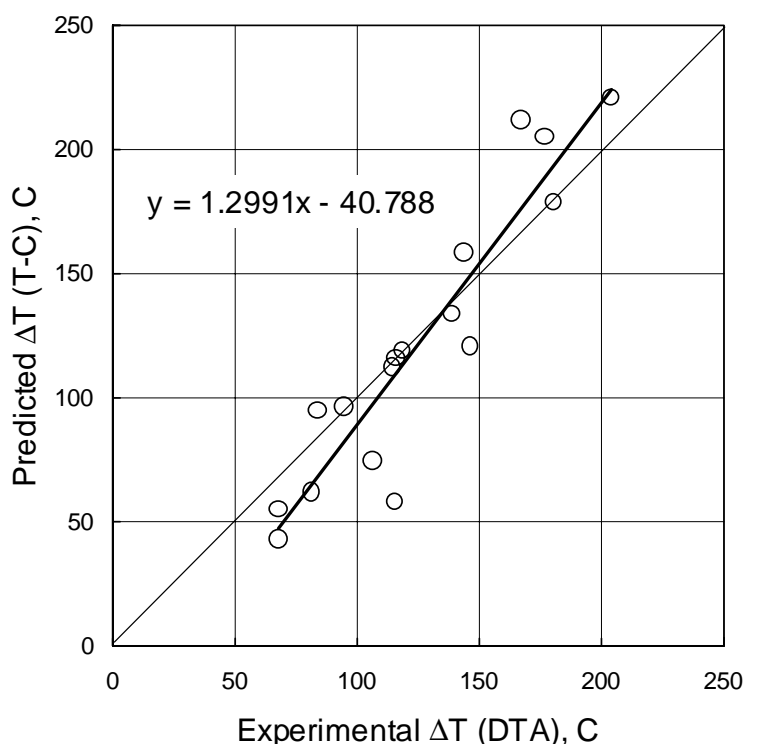

Figure 4-11: Correlation of freezing range data determined by DTA and Thermo-Calc.

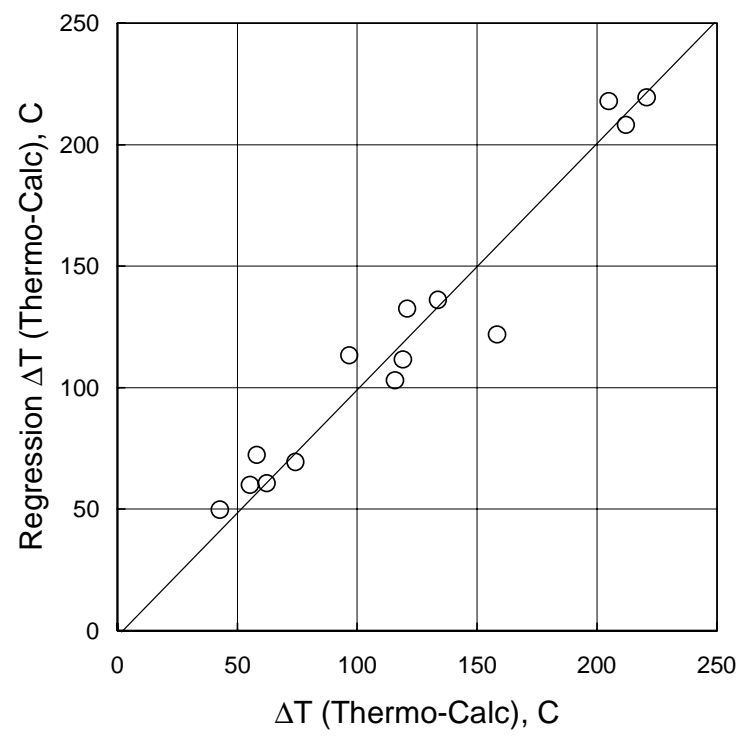

(a)

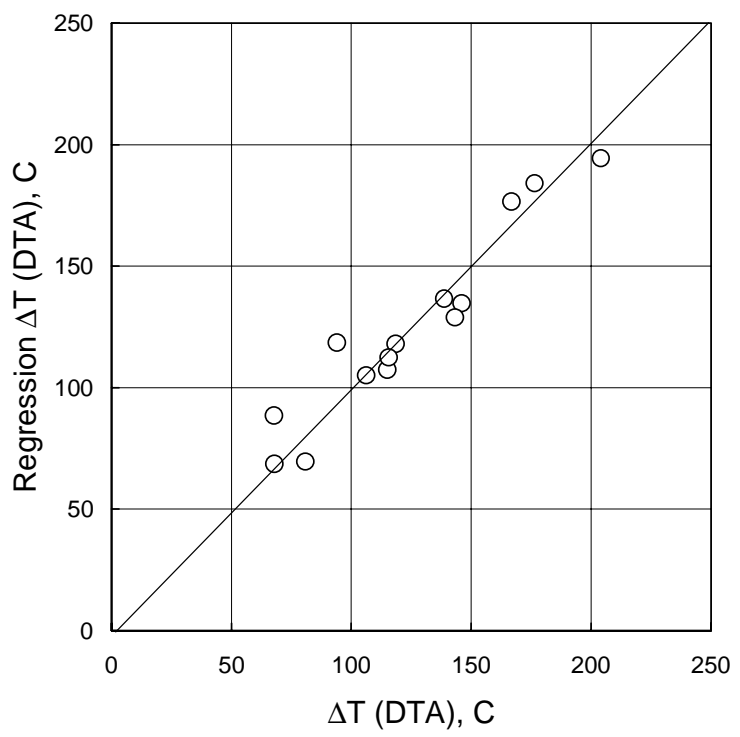

(b)

Figure 4-12: Regression results of freezing range data of Thermo-Calc and DTA. 


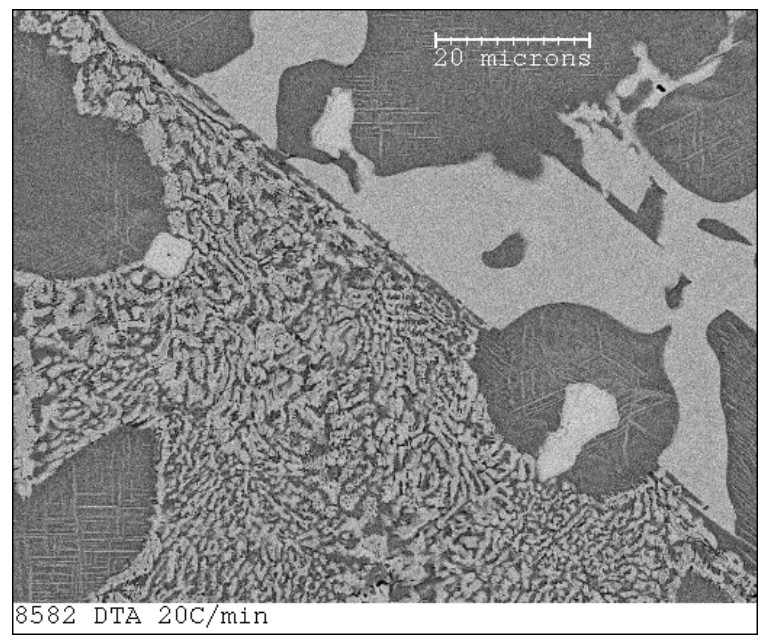

(a)

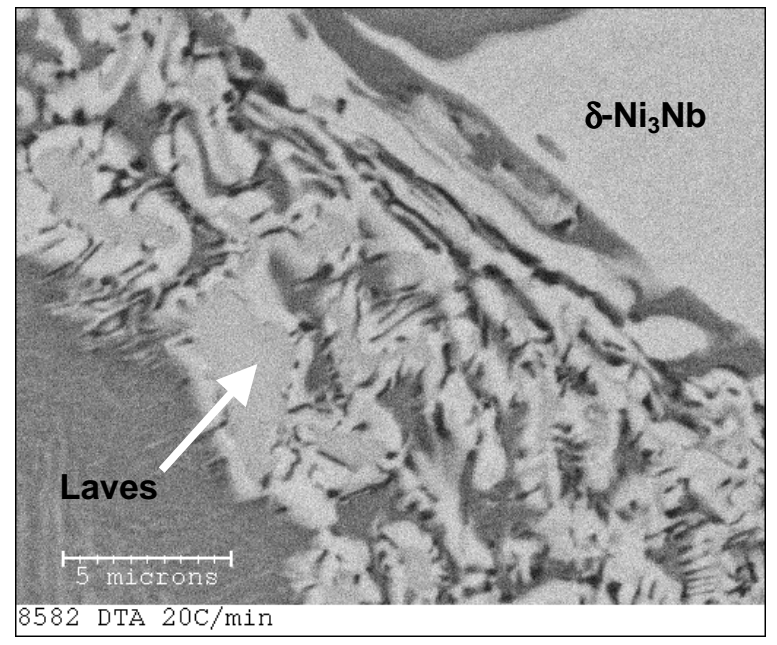

(b)

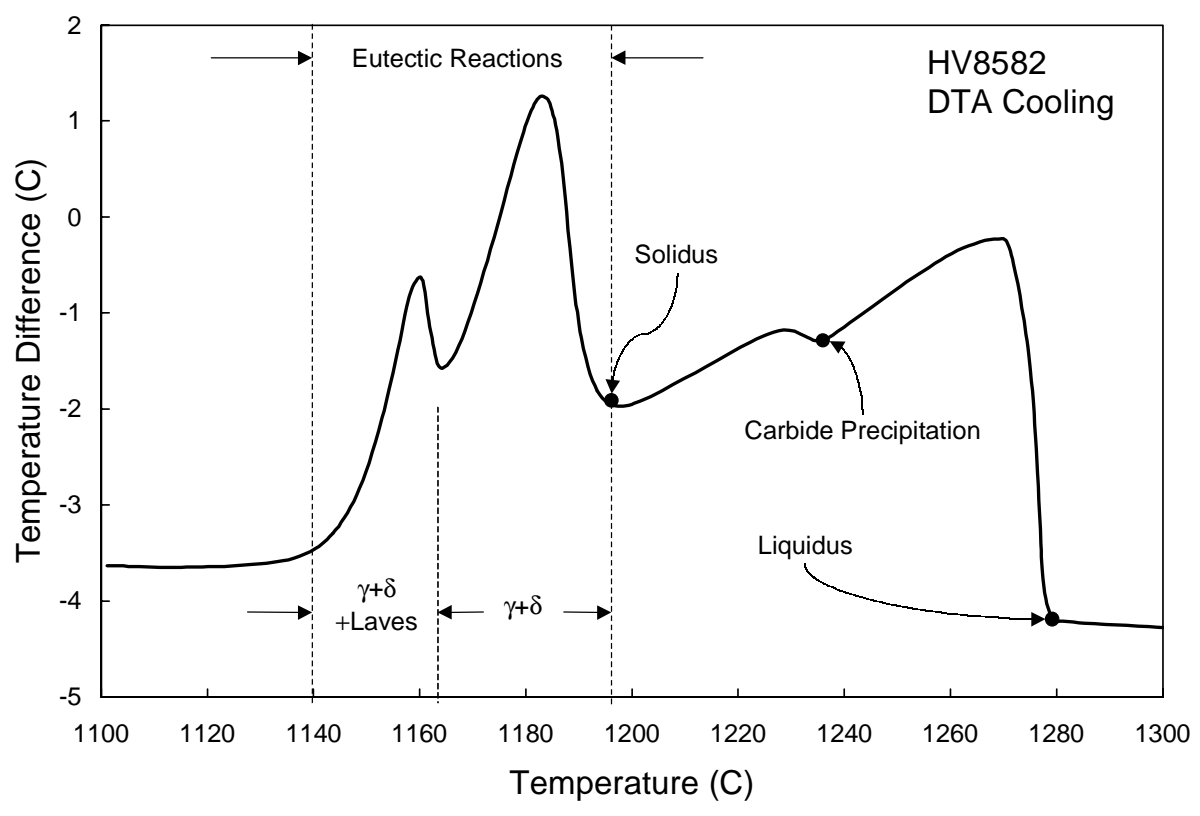

(c)

Figure 4-13 (a) and (b): Back-scattered electron images of alloy HV8582, (c): DTA cooling curve of HV8582 showing two eutectic reactions. 


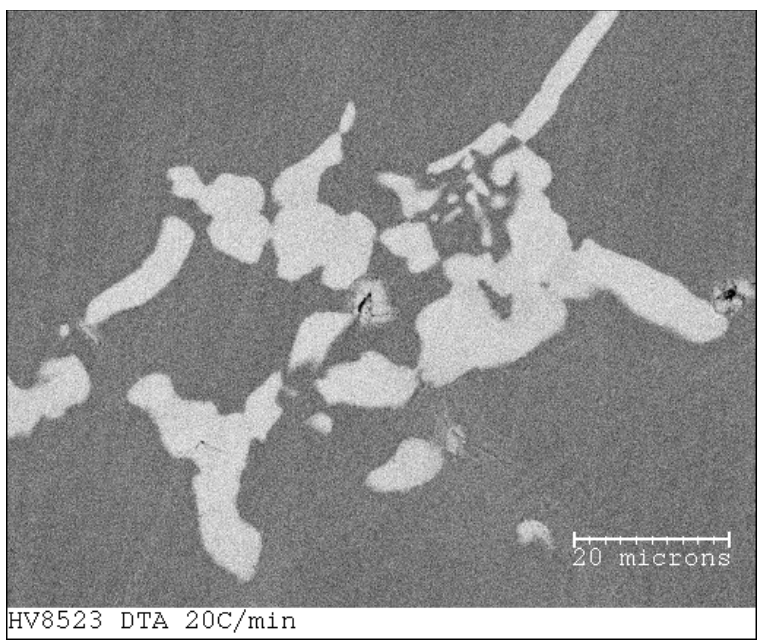

(a)

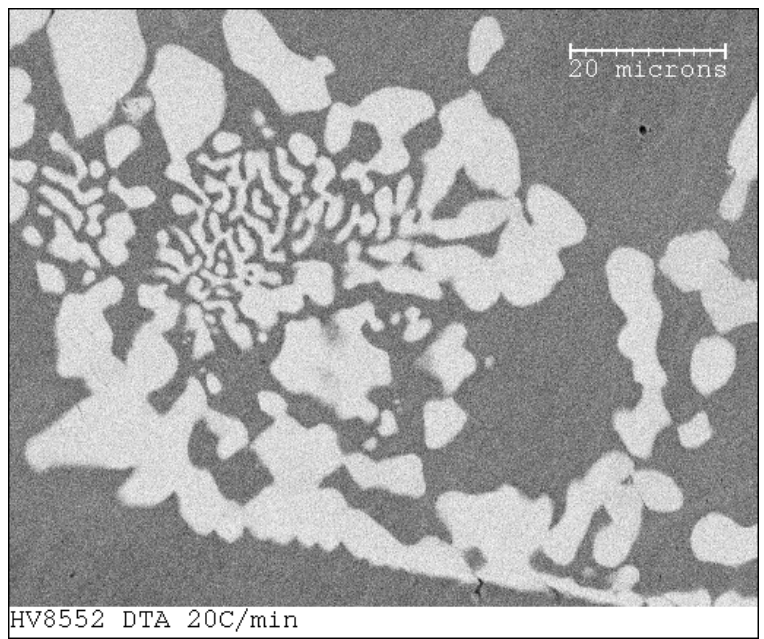

(c)

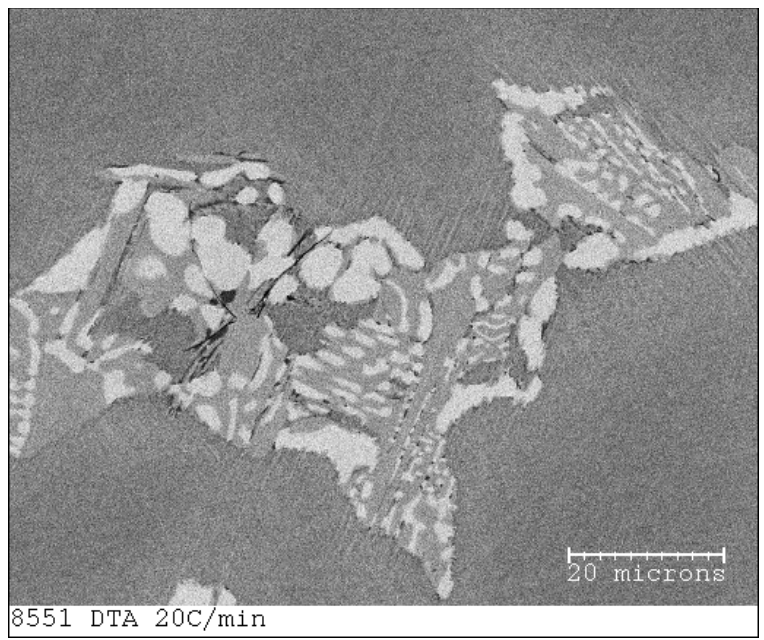

(a)

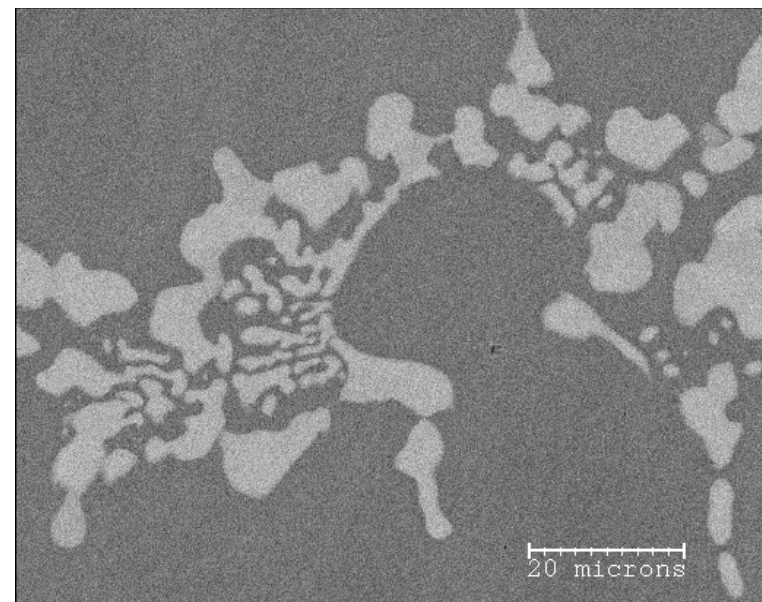

HV8524 DTA $20 \mathrm{C} / \mathrm{min}$

(b)

Figure 4-14: 706 -type of model alloys all exhibit Laves phase as the only eutectic constituent. (a) HV8523, (b) HV8524, and (c) HV8552.

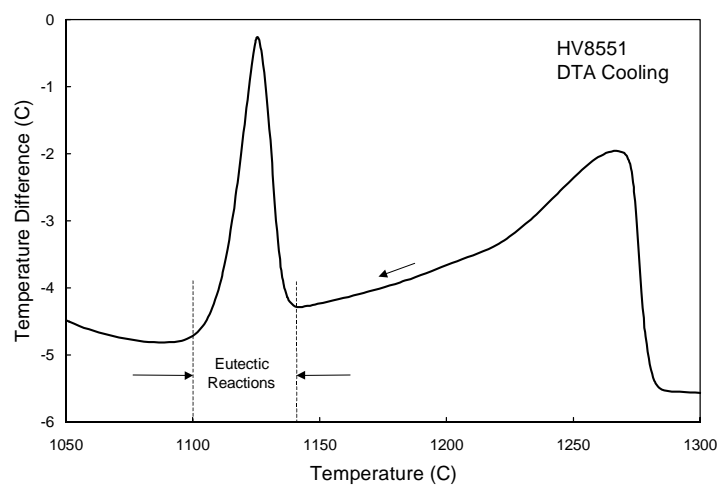

(b)

Figure 4-15: (a) Back-scattered electron image and (b) DTA cooling curve of HV8551. 
Table 4-5: Results of Thermo-Calc simulation of Ni-Cr-Fe-Nb-Ti-Al system

\begin{tabular}{|c|c|c|c|c|c|c|c|c|c|c|c|}
\hline \multicolumn{6}{|c|}{ Composition (wt pet) } & \multirow{2}{*}{$\begin{array}{l}\text { at.\% } \\
\text { Ti/Nb }\end{array}$} & \multicolumn{2}{|c|}{ Eutectic } & \multicolumn{3}{|c|}{ vol. percent } \\
\hline $\mathrm{Cr}$ & $\mathrm{Fe}$ & $\mathbf{N b}$ & $\mathbf{T i}$ & Al & $\mathbf{N b}+\mathbf{T i}$ & & 1 st & $2^{\text {nd }}$ & Laves & Eta & Delta \\
\hline 20 & 18 & 4 & 1 & 0 & 5 & 0.49 & $\delta$ & $\mathrm{L}$ & 1.7 & 0.6 & 3 \\
\hline 20 & 18 & 4 & 3 & 0 & 7 & 46 & $\eta$ & L & 3.5 & 6 & 0.3 \\
\hline 20 & 18 & 4 & 6 & 0 & 10 & 2.91 & $\eta$ & L & 5.6 & 18 & - \\
\hline 20 & 18 & 4 & 9 & 0 & 13 & 4.37 & $\eta$ & L & 6.4 & 35 & - \\
\hline 20 & 18 & 6 & 2 & 0 & 8 & 0.65 & $\mathrm{~L}$ & $\eta$ & 5.3 & 2.3 & 2.9 \\
\hline 20 & 18 & 6 & 4 & 0 & 10 & 1.29 & $\eta$ & L & 8 & 11 & 0.2 \\
\hline 20 & 18 & 6 & 6 & 0 & 12 & 1.94 & $\eta$ & $\mathrm{L}$ & 10.1 & 19.3 & - \\
\hline 20 & 18 & 6 & 9 & 0 & 15 & 2.91 & $\eta$ & L & 12.5 & 37 & - \\
\hline 20 & 18 & 9 & 1 & 0 & 10 & 0.22 & L & $\eta$ & 9.8 & 0.6 & 5 \\
\hline 20 & 18 & 9 & 3 & 0 & 12 & 0.65 & L & $\eta$ & 14 & 6.8 & - \\
\hline 20 & 18 & 9 & 6 & 0 & 15 & 1.29 & L & $\eta$ & 20.7 & 20.8 & - \\
\hline 20 & 18 & 12 & 2 & 0 & 14 & 0.32 & L & $\eta$ & 21 & 2.8 & 3.5 \\
\hline 20 & 18 & 12 & 5 & 0 & 17 & 0.81 & L & $\eta$ & 30 & 14.8 & - \\
\hline 19.93 & 18.25 & 5.33 & 5.55 & 0 & 10.88 & 2.02 & $\eta$ & L & 7.8 & 17.6 & - \\
\hline 19.36 & 18.06 & 7.32 & 5.09 & 0 & 12.41 & 1.35 & $\mathrm{~L}$ & $\eta$ & 11.8 & 17 & - \\
\hline 20 & 18 & 8 & 4 & 0 & 12 & 0.97 & L & $\eta$ & 13 & 11.5 & - \\
\hline 20.02 & 36.04 & 5.39 & 5.43 & 0 & 10.82 & 1.95 & $\mathrm{~L}$ & $\eta$ & 23.8 & 4.5 & - \\
\hline 20.32 & 36.47 & 5.75 & 4.27 & 0 & 10.02 & 1.44 & L & $\eta$ & 21 & 4 & - \\
\hline 19.81 & 35.02 & 7.79 & 3.9 & 0 & 11.69 & 0.97 & L & $\eta$ & 25 & 4 & - \\
\hline 16.06 & 35.37 & 3.23 & 2.94 & 0 & 6.17 & 1.77 & L & $\eta$ & 4.4 & 6 & - \\
\hline 19.94 & 18.09 & 7.72 & 4.01 & 1.12 & 11.73 & 1.01 & $\mathrm{~L}$ & $\eta$ & 12.2 & 7.6 & - \\
\hline 19.19 & 17.38 & 7.41 & 5.32 & 1.13 & 12.73 & 1.39 & $\mathrm{~L}$ & $\eta$ & 14.4 & 11.4 & - \\
\hline 20.73 & 18.83 & 5.62 & 4.24 & 1.18 & 9.86 & 1.46 & L & $\eta$ & 7.7 & 7.9 & - \\
\hline 19.69 & 18.21 & 5.37 & 5.59 & 1.14 & 10.96 & 2.02 & $\mathrm{~L}$ & $\eta$ & 9.1 & 11.9 & - \\
\hline
\end{tabular}


Chapter 5

Composition, Temperature and Fraction Solid in Solidification: Experimental Determination

5.1. Introduction 90

5.2. Solute Redistribution 91

5.2.1. Microsegregation Profiles $\quad 92$

5.2.2. Partition Ratio 93

5.3. Fraction of Solid 94

5.3.1. Quantitative DTA 96

5.3.2. Model Alloys 97

$\begin{array}{ll}\text { 5.3.3. Alloying Effects } & 97\end{array}$

5.4. Secondary Dendrite Arm Spacing 98

5.5. Discussion 99

5.5.1. Kinetic Effects 99

$\begin{array}{lll}\text { 5.5.2. Experiments versus CALPHAD } & 101\end{array}$

$\begin{array}{lll}\text { 5.6. Summary } & 102\end{array}$ 


\subsection{Introduction}

The correlation of liquid composition, temperature, and fraction solidified during the dendritic solidification of superalloys is of great value in understanding macrosegregation phenomena such as freckles. Two theoretical approaches have been applied to predict these attributes. First, for binary alloys whose liquidus has linear relationship with composition, the liquid composition is simply related to fraction solid and temperature by Scheil model. Second, CALPHAD method employs thermodynamic principles to obtain these relations of multicomponent alloys based upon same assumptions as Scheil model, e.g. complete diffusion in liquid that results in uniform liquid concentration and negligible diffusion in solid. Although both treatments provide good guidelines, real solidification of multicomponent alloys often deviate from the theoretical predictions due to the complexity of alloy chemistry and processing conditions.

It is vitally important to develop simple and reliable experimental techniques that can actually measure these attributes in materials obtained from solidification conditions close to real production. However, very few published data is available to meet these requirements. Experimental techniques to investigate the attributes include quantitative metallography, X-ray diffraction analysis, EDS and microprobe measurements. The strategy for application is often cumbersome and time-consuming due to the requirement of strict controls over multiple experiments.

The aim of this work is to develop simple-to-use and accurate experimental strategies to quantitatively determine the detailed solute redistribution profile and fraction solid versus temperature relation in multicomponent alloys. These results, together with the measurement of secondary dendrite arm spacing at the end of this chapter, will provide significant insights for the study of the key control parameters in freckle formation.

\subsection{Solute Redistribution}

The extent of solute redistribution, that is, segregation of solute elements over distances on the order of dendrite arm spacings, can be measured by one of the following: (a) amount of non-equilibrium eutectic, (b) amount of non-equilibrium secondary phase, (c) minimum 
solid composition, (d) ratio of minimum to maximum composition of the primary phase, or (e) composition versus fraction solid profiles, which will be discussed in this section.

\subsubsection{Microsegregation Profiles}

The typical dendritic structure for analysis is shown as a back-scattered electron image whose contrast is determined by the local concentration of heavy elements, such as $\mathrm{Nb}$ (Figure 5-1). The higher the $\mathrm{Nb}$ concentration, the brighter is the contrast. The main features in Figure 5-1 are the primary and secondary dendrites and the interdendritic eutectic phases as $\mathrm{Nb}$ becomes more enriched towards eutectic region. A simplified dendrite model is shown in Figure 5-2. Nb concentration increases monotonically from the dendrite core to the outside layer of eutectic with the progress of solidification, hence, the curve in Figure 5-2 represents the composition profile during solidification. It is possible to determine the same composition profile in a solidified sample by employing the systematic point count method [46].

In the systematic point count method, a test grid with a regular array of test points is superimposed on the image as Figure 5-1. The concentration profile during solidification can be obtained by putting these data in ascending or descending order depending on the partition ratio. In the current study, coordinates of a coarse square-mesh virtual grid were programmed onto the sample surface. Composition at each grid point was measured using a fully automated Energy Dispersive Spectrometer (EDS) attached to the scanning electron microscope (SEM). Other operating conditions are described in chapter

2. The step size (the distance between two consecutive measurements) is approximately the same as the secondary dendrite arm spacing of the specimen (50 100 microns). A total of about 150 measurements were obtained from each sample. Most of the samples used were melted and solidified at $20^{\circ} \mathrm{C} / \mathrm{min}$ in the TA-1600 DTA cell. The data from the EDS measurements were processed using the procedure described in Appendix B to obtain elemental redistribution profiles in solid.

The solid composition profiles of HV8851 (Ni-20Cr-6Ti), HV8852 (Ni-20Cr8.6Nb), and HV8853 (Ni-20Cr-18Fe-7Nb) are shown in Figure 5-3. In these alloys, solid concentrations of $\mathrm{Nb}$ and $\mathrm{Ti}$ increase monotonically with solidification, which results in their enrichments in the interdendritic liquid. Matrix forming elements such as $\mathrm{Cr}$ and $\mathrm{Fe}$ 
prefer to distribute in primary phase, hence, the liquid continues to be depleted of these elements. The average of all of the measurements for each element was taken and compared with nominal alloy composition. The differences between them are given in Table 5-1. It is seen that most of the average values are comparable with the nominal compositions, which means that the measurement obeys the solute conservation assumption. The average concentration of $\mathrm{Nb}$ in HV8853 is much higher that nominal value. This is due to the very few points with extremely high concentrations. They were probably located in the secondary eutectic phases such as $\mathrm{NbC}$ carbide in which the $\mathrm{C}$ content cannot be accurately determined because of equipment limits.

Liquid composition in the interdendritic region can be calculated from the measured solid composition profile. Assuming complete solute mixing in the liquid, the composition of the remaining interdendritic liquid at any fraction solid is given as

$$
C_{L}^{i}=\frac{C_{0}^{i}-\int_{0}^{f_{S}} C_{S}^{i} d f_{S}}{1-f_{S}}
$$

$C_{L}^{i}, C_{S}^{i}$, and $C_{0}^{i}$ are the liquid, solid, and nominal compositions of solute $i$ respectively. $f_{S}$ is the weight fraction of solid and $d f_{S}$ is the differential element of the fraction solidified. It is assumed that no solute transport between the volume element and surrounding so that total weight of solute in the volume equals to the nominal composition throughout solidification.

Figure 5-4 shows the liquid composition profiles from equation (5-1) of HV8851, HV8852, and HV8853. Ti and $\mathrm{Nb}$ are solute elements in these alloys and they both segregate monotonically into the interdendritic liquid during solidification. It should be noted that the liquid composition at the last 10 20\% solidification are not calculated due to the lack of precision of measurement in the interdendritic region.

\subsubsection{Partition Ratio}

When equilibrium exists at the interface during solidification of an alloy, the ratio of concentrations between liquid and solid is known as its equilibrium partition ratio. This ratio provides effective measure of solute segregation. If it is close to unity, then there is very little partitioning of element between liquid and solid. If it is less than unity, there is 
preferential partitioning to the liquid, and vice versa. Therefore, it is essential to have reasonably accurate values of partition ratios for solidification simulations.

In the current study, the partition ratio $k_{i}$ of an element at any given fraction solid $f_{S}$ can be calculated as the ratio of the interpolated interface solid composition to the composition of the remaining liquid obtained by using equation 5-1. Figure 5-5 shows the partition ratios of elements in the three model alloys versus fraction solid. The ratios of $\mathrm{Cr}$ and $\mathrm{Fe}$ remain almost constant and slightly above 1 throughout the solidification. The partition ratio of $\mathrm{Ti}$ increases from 0.68 to 0.88 when $f_{S}$ changes from 0 to 0.8 . $\mathrm{Nb}$ ratio increases from 0.54 to 0.74 with $f_{S}$ changing from 0 to 0.8 in HV8852, and increases from 0.44 to 0.8 with $f_{S}$ changing from 0 to 0.8 in HV8853. It is interesting to note that the solute partition ratios increase during solidification for both $\mathrm{Ti}$ and $\mathrm{Nb}$. Knorovsky et al [54] measured the partition ratio of $\mathrm{Nb}$ in both quenched and solidified welds of alloy 718 . Their result shows that $k_{\mathrm{Nb}}$ is not statistically different between the beginning and the end of solidification and it was assumed to be constant during solidification.

The discrepancy between model alloys and industrial alloys can be attributed to the higher solute concentration and absence of solute interactions in model alloys. Both of $\mathrm{Nb}$-containing model alloys have much higher $\mathrm{Nb}$ contents (HV8852: 8.6 wt pct, HV8853: $7 \mathrm{wt}$ pct) than alloy 718 (5 wt pct). This may correspond to different locations on the phase diagram and result in difference of $k_{\mathrm{Nb}}$. In model alloys, single solute element $(\mathrm{Nb}$ or Ti) is present in the interdendritic liquid. Their solubility in $\gamma$ matrix may increase during solidification due to the lack of competition. However, the change of solubility of individual solute is limited when multiple solutes are present in the system due to their interactions. A parallel analysis of solid, liquid, and partition ratios are conducted on industrial alloys [53] and $k$ ratios of $\mathrm{Ti}$ and $\mathrm{Nb}$ are found to be nearly constant during solidification.

\subsection{Fraction of Solid}

Several important solidification parameters can be extracted from the fraction solid versus temperature curve as shown in Figure 5-6. 
1) The shape of $f_{\mathrm{S}}(T)$ curve during solidification of primary $\gamma$ phase.

2) Freezing range of the alloy $\left(T_{L}-T_{S}\right)$.

3) Total solidification range of the alloy $\left(T_{L}-T_{E}\right)$.

4) Volume fraction of eutectics

Character 1) is of great importance in current study since it can be used in the calculation of permeability. It also provides the link between liquid composition and temperature so that the liquid density can be obtained. Character 2) was previously investigated in chapter 4 and it can be used to determine the depth of mushy zone if the processing condition is known. Character 3) is important to determine the incipient melting point. If the total range is large, the alloy may have a low incipient melting point that is critical in forging and heat treatment. Character 4) represents the extent of solidification. 3) and 4) are not studied in this study.

The major approach for obtaining $f_{\mathrm{S}}(T)$ curve is through interrupt quenching of semi-solid samples during equiaxed or directional solidification. Area fractions are measured and correlated with quenching temperatures. The method is time-consuming and requires strict environmental protection due to the reactive nature of transition elements. The purpose of the present section is to develop a quantitative approach used for the determination of the $f_{\mathrm{S}}(T)$ curves from DTA data. The method is very attractive due to its accuracy and the reduction of required analysis time. Results of model alloys are verified using quenching data and exhibits general agreements.

\subsubsection{Quantitative DTA}

The transformation rate during solidification of an alloy, $d f_{S} / d t$, can be used to determine the relation between fraction solidified and transformation temperature under a constant cooling rate. Traditionally, the information can only be obtained by measuring the volume fraction of solidified area in sample whose structure has been frozen by quenching during solidification cycle. Differential Thermal Analysis (DTA) provides valuable information about the rate of growth and characteristic temperatures during solidification. A theoretical treatment of DTA is developed in the current study to determine evolution of fraction of solid with respect to solidification time and temperature. 
The determination of solidification rate from thermal analysis is based on the fact that the amount of heat supplied to, or carried away from a sample is directly proportional to the solidification rate. In the DTA setup schematically shown in Figure 5-7, the following heat balance applies [47]:

$$
\lambda\left(C_{P R} W_{P R}-C_{P S} W_{P S}\right)+\frac{d f_{S}}{d t}(-\Delta H) W_{S}=\frac{d Q}{d t}
$$

Where $d Q / d t$ is the amount of of heat carried away from the sample during solidification, $\lambda$ is the reference cooling rate $\left({ }^{\circ} \mathrm{C} / \mathrm{min}\right), C_{P R}$ and $C_{P S}$ are the heat capacities of the reference and sample materials $\left(\mathrm{cal} / \mathrm{g}{ }^{\circ} \mathrm{C}\right), W_{R}$ and $W_{S}$ are the reference and sample weights $(\mathrm{g}),(-\Delta H)$ is the sample latent heat of solidification (cal/g), and $d f_{S} / d t$ is the sample solidification rate $\left(\mathrm{min}^{-1}\right)$. The first term on the left side of equation (5-2) represents the transformation-free term and the second one is related to solidification. In the temperature range of solidification in Ni-based superalloys $\left(1400^{\circ} \mathrm{C} \sim 1200^{\circ} \mathrm{C}\right)$, radiation heat transfer is the dominant mode of heat loss from the sample to the surrounding environment. The following equation is applied:

$$
\frac{d Q}{d t}=A \sigma \varepsilon\left(T_{s}^{4}-T_{f}^{4}\right)
$$

where $A$ is the area of the sample, $\sigma$ is the Stefan-Boltzman constant, $\varepsilon$ is the emissivity of sample material, $T_{S}$ and $T_{f}$ are the sample and furnace temperatures respectively. In this study, $T_{f}$ is assumed to be equal to the reference temperature, $T_{R}$. Since $T_{S} / T_{f} \approx 1$, equation (5-3) can be simplified to

$$
\frac{d Q}{d t}=A \sigma \varepsilon\left(T_{S}^{4}-T_{f}^{4}\right)=A \sigma \varepsilon \cdot 4 T_{R}^{3}\left(T_{S}-T_{R}\right)=\gamma \cdot T_{R}^{3} \Delta T^{*}
$$

where $\gamma=4 A \sigma \varepsilon$ and $\Delta T^{*}=T_{S}-T_{R}$.

If there is no transformation, the baseline heat balance can be described as

$$
\begin{aligned}
& \lambda\left(C_{P R} W_{P R}-C_{P S} W_{P S}\right)=\gamma T_{R}^{3} \cdot \Delta T_{B L} \\
& \Delta T_{B L}=\frac{\lambda\left(C_{P R} W_{P R}-C_{P S} W_{P S}\right)}{\gamma T_{R}^{3}}
\end{aligned}
$$

Then the latent heat of solidification can be obtained by integrating equation (5-2) from the beginning (time $=0$ ) to the completion of solidification (time $=t_{\mathrm{L}}$ ) assuming constant $\lambda$ : 


$$
\Delta H=\int_{0}^{t_{L}}\left(\Delta T^{*}-\Delta T_{B L}\right) \cdot \frac{\gamma T_{R}^{3}}{W_{S}}=\int_{0}^{t_{L}} \Delta T^{\prime} \cdot \frac{\gamma T_{R}^{3}}{W_{S}} d t
$$

where $\Delta T^{\prime}$ is the baseline-corrected temperature difference.

Solid fraction at any time and temperature can then be calculated by

$$
f_{S}\left(t^{\prime}\right)=\frac{1}{\Delta H} \int_{0}^{t^{\prime}}(\Delta T)^{\prime} \frac{\gamma T_{R}^{3}}{W_{S}} d t
$$

The constant $\gamma$ can be estimated by calibrating the equipment with pure $\mathrm{Ni}$, whose total heat of solidification and liquidus temperature are known.

\subsubsection{Model Alloys}

Fraction solidified versus temperature relations in model alloys are obtained using the method as described in previous section and the results are shown in Figure 5-8 through Figure 5-11. For the purpose of comparison, the $f_{\mathrm{S}}(T)$ curves predicted by Thermo-Calc are also given.

The quantitative DTA analysis and Thermo-Calc prediction exhibit good consistence in the relative position of fraction solid at any given temperature for all of the model alloys considered. For example, DTA analysis shows that HV8549 has higher fraction solid and wider solidification range than HV8520, which is in agreement with those predicted by Thermo-Calc. Excessive eutectics in model alloys that are observed in the experimental alloys are successfully predicted using DTA approach. The difference between the absolute values of fraction solid is that because of the intrinsic difference between Thermo-Calc methodology and experimental conditions. This will be discussed in section 5.5 .

The quantitative DTA analysis is also compared with interrupt quenching results obtained for industrial alloys [53]. These results were obtained by measuring the area fraction of solid in samples that have been quenched from different temperatures during solidification. Both manual point counting method and automated image analysis were used to measure the volume fractions of solid. Modified DTA setup was used for melting the sample so the temperature can be controlled within $\pm 2{ }^{\circ} \mathrm{C}$. The quenched sample has

the approximately the same size as a DTA sample. Please refer to pages 33 to 36 of reference [53] for detailed description and accuracy of the quenching test. The effect of 
back diffusion in solid is prohibited in the interrupt quenching experiment. Results of industrial alloys 718 and 625 from quenching experiment are compared with those obtained using quantitative DTA method as shown in Figure 5-12. Fraction of solid data using quantitative DTA analysis is closer to quench experiments than those predicted by Thermo-Calc, especially at later stage of solidification. It is noted that alloy 718 exhibits lower fraction solid than alloy 625 using all of the three approaches provided no solid diffusion and complete liquid mixing.

The relative good agreement between quantitative DTA analysis and other techniques indicates that DTA analysis can be used as a simple and reliable method to determine $f_{\mathrm{S}}(T)$ curve during solidification. The discrepancy among the absolute values obtained using different methods is of reasons other than composition effect. More work is needed to study these effects. Therefore the quantitative DTA method provides a simple way to study composition effect on the fraction solid evolution during solidification for different alloys. The method offers advantages of time-saving and accuracy as compared with quenching test. Detailed study of the effects of various properties in equation (5-2) is needed in the future to improve the approach.

\subsubsection{Alloying Effects}

Various studies indicate that freckles are likely to initiate at mushy zone locations correspond to a liquid fraction of the order of 0.4 0.6 where fluid flow leading to freckles will develop more easily in regions of higher permeability. In industrial freckle-prone alloys, such as 718 and 625 , eutectic reactions only occur when $80 \sim 90 \%$ of the liquid have been solidified. Thus, it is important to determine the alloying effects on the fraction solid evolution curve during the primary phase solidification.

The fraction of solid versus temperature relation affects the permeability in two folds, e.g. the absolute value of $f_{\mathrm{s}}$ and the slope of $f_{\mathrm{s}}(T)$ curve. Mathematically, these effects are represented by a single parameter, $\alpha$, since the primary solidification section of the $f_{\mathrm{s}}(T)$ curve can be fitted using exponential equation, $f_{L}=e^{-\alpha \cdot \Delta T}$, where $\Delta T=T_{L}-T$. Example of alloy HV8853 is shown in Figure 5-13. Since the exponential curves always originate from 1, the pre-exponential coefficient is assumed to be 1 . The higher the $\alpha$, the greater is $f \mathrm{~s}$, providing the same temperature difference from liquidus. The $\alpha$ values of 
model alloys and three industrial alloys obtained from $f_{\mathrm{s}}(T)$ of quantitative DTA analysis are given in Table 5-2.

Linear regression analysis is conducted to correlate $\alpha$ with nominal alloy composition. $\mathrm{Cr}, \mathrm{Fe}, \mathrm{Al}, \mathrm{Nb}$, and $\mathrm{Ti}$ concentrations are used for regression. However, only $\mathrm{Nb}$ and $\mathrm{Ti}$ are found to be statistically significant. The equation is given as follows:

$$
\alpha=-0.0397+0.002836 \cdot[\mathrm{Nb}]+0.002131 \cdot[\mathrm{Ti}]
$$

The adjusted $\mathrm{R}$ square value of the regression analysis is 0.814 . $P$ values of the coefficients of $\mathrm{Nb}$ and $\mathrm{Ti}$ are 0.0008 and 0.0014 respectively. ( $\mathrm{P}$ value is statistical factor used in hypothesis test to indicate statistical significance, $\mathrm{P}<0.05$ means good statistical significance.) Terms in brackets are the alloy composition in wt pct. The statistics of the equation are significant, the regression exhibits strong correlation between response and variables. The dependence of $\alpha$ on $\mathrm{Nb}$ and Ti concentrations are shown in Figure 5-14. Comparison between predicted and actual values of $\alpha$ is shown in Figure 5-15. Equation (5-9) shows that $\mathrm{Nb}$ addition has more pronounced effect in reducing fraction solid than Ti. No apparent effects of $\mathrm{Cr}$ and Fe can be found on $\alpha$. Equation (5-9) applies to Ni-20Cr18 (or 36)Fe-Nb-Ti-1Al system where the composition ranges are: $3<[\mathrm{Nb}]<7.8$ and $0.3<[\mathrm{Ti}]<5.6$, all in wt pct. It is noted that the equation also applies to industrial alloys such as alloys 718, 706, and 625 .

\subsection{Secondary Dendrite Arm Spacing}

Permeability depends on the primary and secondary dendrite arm spacings as well as the fraction of solid in the mushy zone. It has been found [8] that poor heat extraction during remelting process results in longer local solidification time, which produces large dendrite arm spacings that freckles are easier to flow. It is generally agreed that processing conditions dictate these characteristic lengths. No systematic study has been reported in literature regarding composition effect on dendrite arm spacings in superalloys. In order to develop acceptable freckling criteria, the role of composition in the evolution of dendritic spacings is investigated in the present study.

Secondary dendrite arm spacings, $d_{2}$, are measured in model alloys solidified at the same cooling rate of $20^{\circ} \mathrm{C} / \mathrm{min}$ in DTA. It is intended to eliminate the processing effect, 
thus, to study the composition effects on the dendritic structure, such as fraction solidified as well as dendritic spacings. These samples were solidified in an equiaxed manner so that only $d_{2}$ can be measured. The results are given in table 5-2 and plotted against the $\mathrm{Nb}, \mathrm{Ti}$, and $\mathrm{Ti} / \mathrm{Nb}$ ratio for each model alloy systems in Figure 5-16. The error bars are +/standard deviations of measurements.

In Ni-20Cr-18Fe-Nb-Ti model alloys, $d_{2}$ increases from $37 \mu \mathrm{m}$ to $45 \mu \mathrm{m}$, as the $\mathrm{Ti} / \mathrm{Nb}$ ratio changes from 1.35 to 2 , with a maximum standard deviation of $5.4 \mu \mathrm{m}$. When the Fe level is $36 \mathrm{wt}$ pct in the system, $d_{2}$ increases from $34 \mu \mathrm{m}$ to $40 \mu \mathrm{m}$, as the $\mathrm{Ti} / \mathrm{Nb}$ ratio increases from 1 to 2 . The maximum standard deviation of measurement is $4.7 \mu \mathrm{m}$. When 1 wt pct $\mathrm{Al}$ is added to the system, no significant change of $d_{2}$ with $\mathrm{Ti} / \mathrm{Nb}$ ratio can be

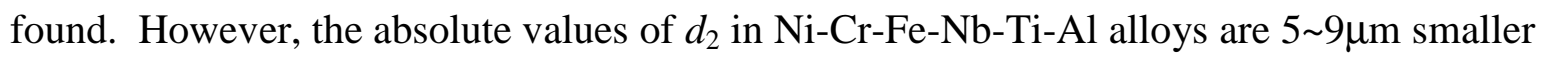
than alloys without $\mathrm{Al}$ additions at $\mathrm{Ti} / \mathrm{Nb}$ ratios of 1 or higher. The differences in $d_{2}$ are higher than their corresponding standard deviations of measurements, hence, the observations are statistically significant. No conclusion of $\mathrm{Al}$ effect can be drawn due to the lack of data. Plots of $d_{2}$ against $\mathrm{Nb}$ and Ti are shown in Figure 5-16 (b) and (c). No apparent trend can be found in these graphs.

It is difficult to experimentally determine the composition effect on $d_{2}$ due to the amount of measurements involved. Result included in the current study is preliminary, which means no firm conclusion can be drawn. However, result of $d_{2}$ in model alloys RN904 and RN903 (horizontal solidification, chapter 3) indicates that composition effect does exist and needs further investigation.

\subsection{Discussion}

\subsubsection{Kinetic Effects}

\subsubsection{Solid-state diffusion effect}

The solute redistribution model developed by Brody and Flemings [48] can be used to estimate the significance of solid-state diffusion during solidification, which, for a parabolic growth rate, is given by

$$
C_{S}^{*}=k C_{0}\left[1-(1-2 \alpha k) f_{s}\right]^{\frac{k-1}{1-2 \alpha k}}, \alpha=\frac{D_{S} t_{f}}{L^{2}}
$$


Here, $k$ is the equilibrium distribution coefficient, $D_{S}$ is the diffusivity of solute in solid (assumed constant in the derivation of the equation), $t_{f}$ is the local solidification time, $L$ is half the dendrite arm spacing. The back-diffusion potential depends on the diffusion distance $(L)$, the time available for diffusion $\left(t_{f}\right)$, and the diffusivity in the solid ( $\left.D_{S}\right)$.

For a DTA Sample of alloy 718 with $20^{\circ} \mathrm{C} / \mathrm{min}$ cooling rate, the local solidification time $t_{f}$ equals to $600 \mathrm{sec}$ with $200^{\circ} \mathrm{C}$ solidification range. The average secondary dendrite arm spacing is about 100 microns, thus, $L=50$ microns. For the major solute element $\mathrm{Nb}$, $D_{S}=7.5 \times 10^{7} \exp \left(2.64 \times 10^{5} / R T\right)$ micron $2 /$ second. The value of $\alpha$ calculated for $\mathrm{Nb}$ diffusion yields values of 0.05 and 0.003 at $1337^{\circ} \mathrm{C}$ and $1137^{\circ} \mathrm{C}$, respectively. Since the values of $\alpha<<1, \mathrm{Nb}$ diffusion in the solid can be neglected under the solidification conditions. The liquid composition of quenched 718 samples was compared with the ascalculated liquid composition and they match to each other very well. The dendrite core compositions of quenched samples at various temperature drops are similar, which also indicates that the diffusion of $\mathrm{Nb}$ is negligible in the solid during solidification. Similar results were found for other alloying elements. In conclusion, effect of solid-state diffusion on the solid composition profile can be neglected under the applied experimental conditions.

\subsubsection{Cooling Rate Effect}

Cooling rate may affect the solute distribution profile, as well as the fraction of eutectic formed in solidification. It has been shown in the previous section that back-diffusion in solid is negligible under the currently employed cooling condition.

Faster cooling rates will introduce certain degree of undercooling before nucleation. The classical treatment describes the undercooling as, $\Delta T=M_{L}\left(C_{0}-C_{t}\right)$, where $C_{t}$ is the composition of the liquid ahead of dendrite tip, $M_{L}$ is the slope of the liquidus line in the phase diagram. The fraction eutectic, $f_{E}$, can then be calculated using

$$
f_{E}=\left(1-f_{S}^{0}\right) \cdot\left[\frac{C_{S}^{\max }}{k C_{t}}\right]^{1 /(k-1)}, \quad f_{S}^{0}=\frac{C_{t}-C_{0}}{C_{t} \cdot(1-k)}
$$


In the above equations, $C_{S}^{\max }$ is the maximum solid solubility and $f_{S}^{0}$ is the fraction of solid formed at tip temperature. Please see reference (Sarreal and Abbaschian, 1986, [49]) for development of equations.

In DTA test of alloy 718 with a cooling rate of $20^{\circ} \mathrm{C} / \mathrm{min}, \Delta T=10^{\circ} \mathrm{C}$ was encountered. Using the values of $k=0.46, C_{S}^{\max }=9.3 w t . \%$ for $\mathrm{Nb}$, the fraction eutectic ranges from 0.09 to 0.10 for undercooling ranging from $5^{\circ} \mathrm{C}$ to $15^{\circ} \mathrm{C}$ respectively, which is close to measured fraction eutectic of 0.07-0.11 [54]. It is clear that the cooling rate effect on the fraction of eutectic formed under the applied solidification conditions is negligible.

\subsubsection{Experimental versus CALPHAD}

It is beyond the scope of the present study to validate the applicability of CALPHAD method in solidification segregation study. However, since various results have been generated via both CALPHAD and experimental methods, it is worthwhile to phenomenally compare the experimental data with CALPHAD prediction. The comparison is made clear by the following table (Table 5-3). Good correlation in Table 5-3 means that if alloy 1 exhibits higher value than alloy 2 in experimental observation, the same trend will be found in CALPHAD prediction.

Table 5-3: Correlation of CALPHAD and experimental results.

\begin{tabular}{lccc}
\hline \multicolumn{1}{c}{ Solidification Study } & Good & Not Good & Ref. \\
\hline Solidification diagram & $\sqrt{ }$ & & Ch.4 \\
Solidification sequence & $\sqrt{ }$ & & Ch.4 \\
Secondary phases & $\sqrt{ }$ & & Ch.4 \\
Freezing range & $\sqrt{ }$ & & Ch.4 \\
Fraction of solid & $\sqrt{ }$ & $\sqrt{ }$ & Ch.5 \\
Solid and liquid composition & & $\sqrt{ }$ & Ch.5 \& [2] $\&[2]$ \\
Partition ratio & & & \\
\hline
\end{tabular}

Comparison is based on results in model Ni-based alloys and industrial alloys 718, 706, and 625. The temperature data of experimental alloys were obtained using DTA and the composition was measured using Energy Dispersive Spectrometry. DTA uses continuous cooling scheme which means that the accuracy of temperature data depends on the magnitude of undercooling and the sensitivity of the equipment to pick up reaction 
temperatures. It becomes more difficult to discern a reaction temperature if the amount of heat output for a specific reaction (such as eutectic reaction) is small or several reactions overlap in their temperatures. However, the DTA equipment employed in the current study is considered to be relatively accurate. It is shown that CALPHAD performs relatively well in the prediction of phase equilibrium. However, the absolute values of prediction, especially the solid and liquid compositions, always deviate from those found in experiments. This can be attributed to solid-state diffusion, dendrite coarsening, localized solute transport by liquid convection, solute concentrations, higher order $(>3)$ solute interactions, and undercooling, etc. Hence, it is more suitable to use CALPHAD method for comparison between different alloys than to apply it directly in real practice.

\subsection{Summary}

Results and summary in chapter 5 can be summarized as follows:

1. A statistically significant systematic point count technique is developed to determine the composition profiles during solidification of multicomponent alloys.

2. Segregation profiles of model alloys HV8851, HV8852, and HV8853 are obtained. Result shows that $\mathrm{Nb}$ and Ti redistribute preferentially into the interdendritic liquid.

3. The partition ratios of $\mathrm{Nb}$ and $\mathrm{Ti}$ increase with the progress of solidification.

4. Quantitative DTA analysis method is developed to determine $f_{\mathrm{s}}(T)$ relation in superalloys. The method is simple and accurate as compared with quenching test.

5. $f_{\mathrm{S}}(T)$ curves of model alloys are determined using quantitative DTA analysis.

6. The primary phase solidification curve can be fitted using $f_{L}=e^{-\alpha \cdot \Delta T}$ equation.

7. Composition effects on $f_{\mathrm{s}}(T)$ relation can be obtained by correlating $\alpha$ with composition variables. The following equation is obtained using regression analysis for Ni-Cr-Fe-Nb-Ti-Al alloys: $\alpha=-0.0397+0.002836 \cdot[\mathrm{Nb}]+0.002131 \cdot[\mathrm{Ti}]$.

8. No clear relation between alloying addition and secondary dendrite arm spacing can be found in the current study. More work is needed in the future.

9. CALPHAD prediction is suitable for comparison between different alloys. However, it is cautioned to use the method directly in real practice. 


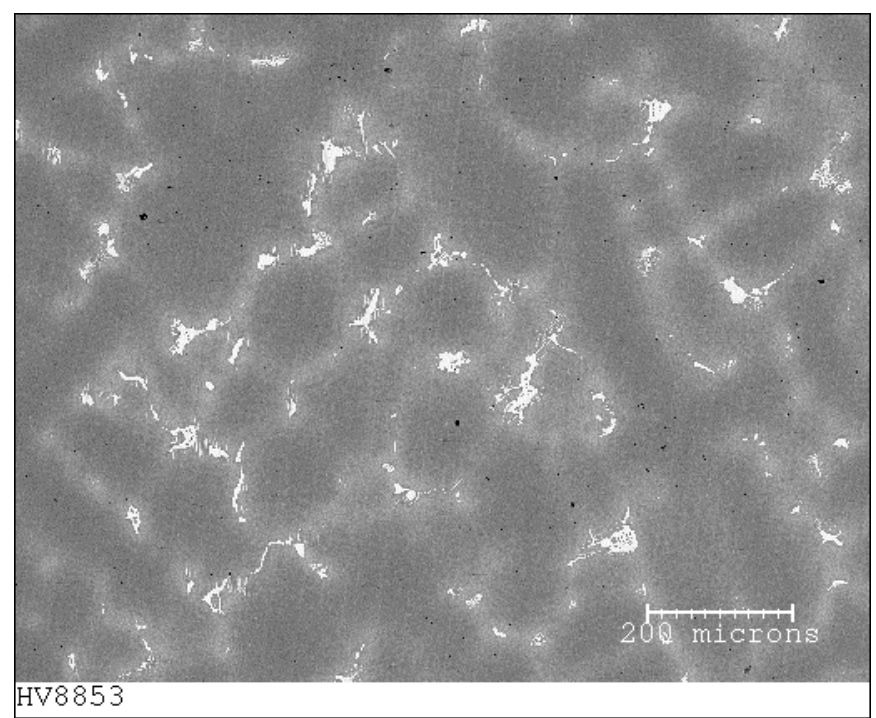

Figure 5-1: Dendrite structure in Ni-based superalloys (Back-scattered electron image).

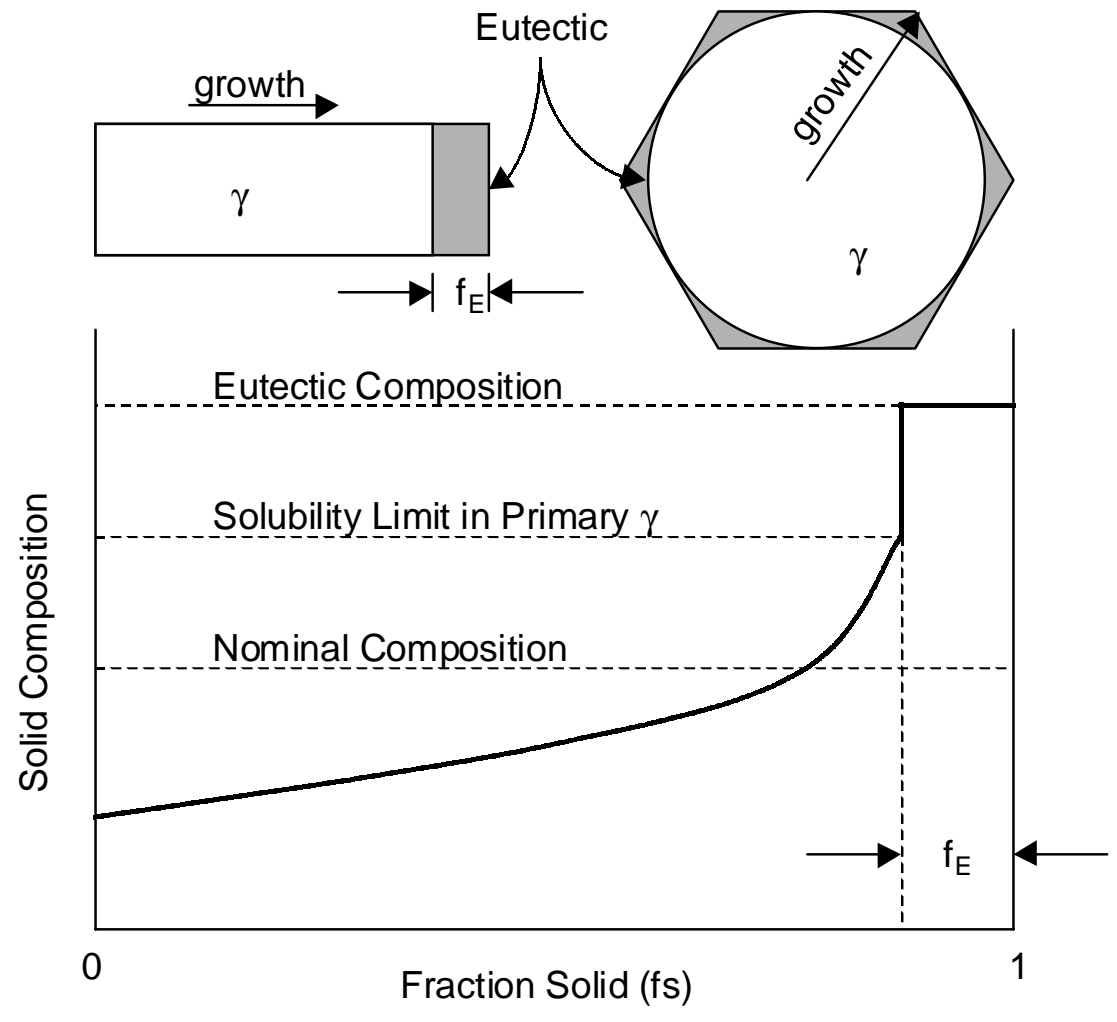

Figure 5-2: Schematic of monototnic increase of solid $\mathrm{Nb}$ concentration in model alloys. $* \mathrm{f}_{\mathrm{E}}$ is the volume fraction of eutectic constituents. 
Table 5-1: Average solid composition in systematic point count measurements. (wt\%) * Values in parenthesis is the standard deviation of EDS measurements.

\begin{tabular}{|c|c|c|c|c|c|}
\hline \multicolumn{2}{|c|}{ Nominal (8851) } & \multicolumn{2}{|c|}{ EDS Average } & \multicolumn{2}{|c|}{ Difference $(\sigma)$} \\
\hline $\begin{array}{c}\mathrm{Cr} \\
19.89\end{array}$ & $\begin{array}{c}\mathrm{Ti} \\
5.94\end{array}$ & $\begin{array}{c}\mathrm{Cr} \\
19.99\end{array}$ & $\begin{array}{c}\mathrm{Ti} \\
6.03\end{array}$ & $\begin{array}{c}\mathrm{Cr} \\
-0.10(0.45\end{array}$ & $\begin{array}{c}\mathrm{Ti} \\
-0.09(0.30)\end{array}$ \\
\hline
\end{tabular}

\begin{tabular}{|c|c|c|c|c|c|}
\hline \multicolumn{2}{|c|}{ Nominal (8852) } & \multicolumn{2}{|c|}{ EDS Average } & \multicolumn{2}{|c|}{ Difference $(\sigma)$} \\
\hline $\begin{array}{c}\mathrm{Cr} \\
20.13\end{array}$ & $\begin{array}{c}\mathrm{Nb} \\
8.36\end{array}$ & $\begin{array}{c}\mathrm{Cr} \\
20.80\end{array}$ & $\begin{array}{c}\mathrm{Nb} \\
7.97\end{array}$ & $\begin{array}{c}\mathrm{Cr} \\
-0.67(0.45)\end{array}$ & $\begin{array}{c}\mathrm{Nb} \\
0.39(0.33)\end{array}$ \\
\hline
\end{tabular}

\begin{tabular}{ccc|ccc|ccc}
\hline \multicolumn{2}{c|}{ Nominal (8853) } & \multicolumn{3}{c|}{ EDS Average } & \multicolumn{3}{c}{ Difference $(\sigma)$} \\
\hline $\mathrm{Cr}$ & $\mathrm{Fe}$ & $\mathrm{Nb}$ & $\mathrm{Cr}$ & $\mathrm{Fe}$ & $\mathrm{Nb}$ & $\mathrm{Cr}$ & $\mathrm{Fe}$ & $\mathrm{Nb}$ \\
20.18 & 17.73 & 6.94 & 19.84 & 17.31 & 7.67 & $0.34(0.45)$ & $0.42(0.43)$ & $-0.73(0.31)$ \\
\hline
\end{tabular}

Table 5-2: $\alpha$ and $d_{2}$ of model alloys

\begin{tabular}{cccccc}
\hline Alloys & Nb & Ti & $\boldsymbol{\alpha}$ & $\boldsymbol{d}_{\mathbf{2}}(\boldsymbol{\mu m})$ & $\boldsymbol{\sigma}(\boldsymbol{\mu m})$ \\
\hline 8851 & 0 & 6 & -0.0413 & - & - \\
8852 & 8.36 & 0 & -0.0277 & - & - \\
8853 & 7 & 0 & -0.0191 & - & - \\
8549 & 5.33 & 5.55 & -0.0119 & 44.66 & 5.37 \\
8520 & 7.32 & 5.09 & -0.013 & 37.31 & 5.04 \\
8550 & 7.72 & 4.01 & -0.0115 & 39.20 & 6.65 \\
8522 & 7.41 & 5.32 & -0.0096 & - & - \\
8521 & 5.62 & 4.24 & -0.0137 & 35.05 & 2.33 \\
8551 & 5.37 & 5.59 & -0.0123 & 35.37 & 2.49 \\
8552 & 7.76 & 3.88 & -0.0076 & 33.59 & 2.81 \\
8523 & 5.75 & 4.27 & -0.0097 & 41.10 & 4.69 \\
8524 & 5.39 & 5.43 & -0.0091 & 40.19 & 0.89 \\
8586 & 3.23 & 2.94 & -0.0302 & - & - \\
718 & 5.41 & 0.98 & -0.0198 & - & - \\
706 & 2.98 & 1.7 & -0.0252 & - & - \\
625 & 3.53 & 0.29 & -0.0317 & - & - \\
\hline
\end{tabular}

Note: $\boldsymbol{\sigma}$ is the standard deviation of $\boldsymbol{d}_{\mathbf{2}}$ measurement. 


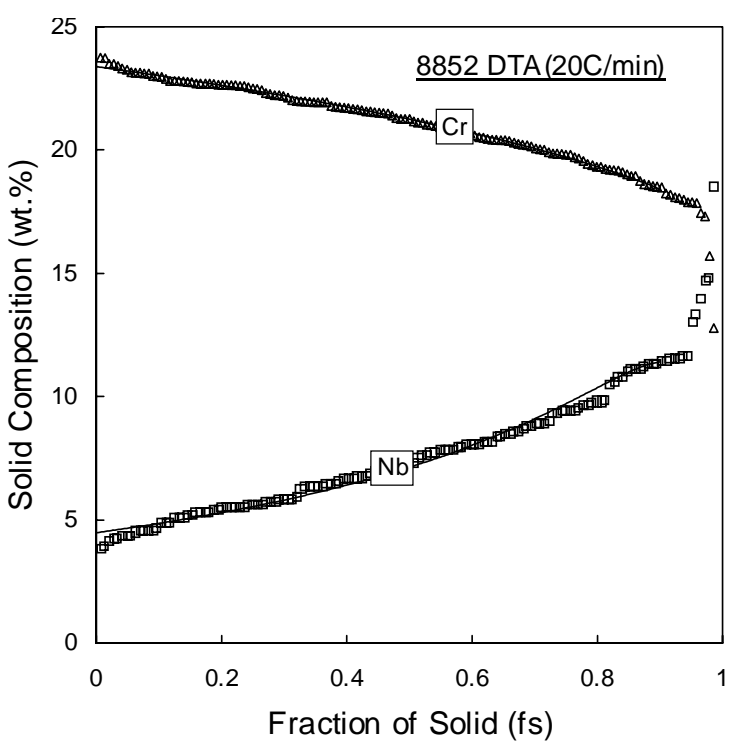

(a)

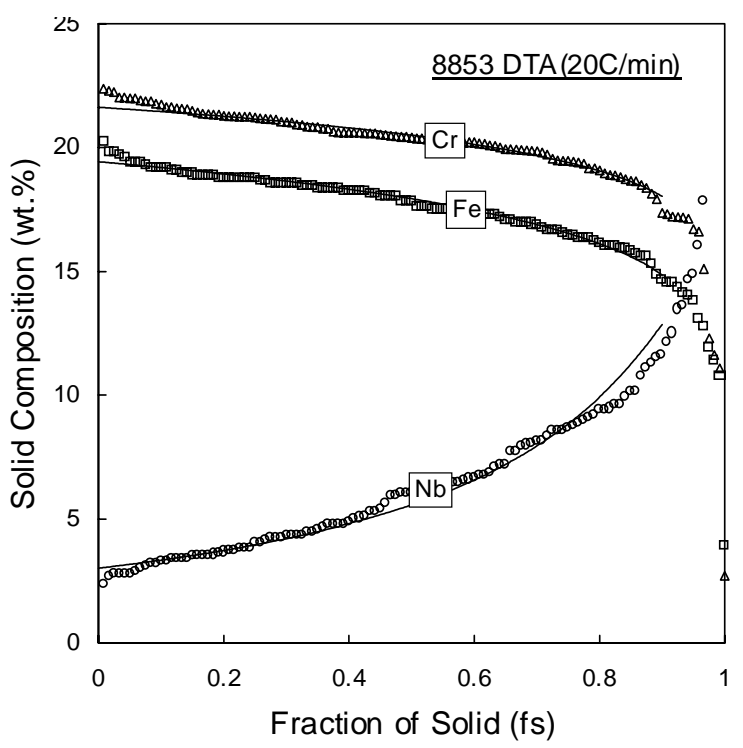

(c)

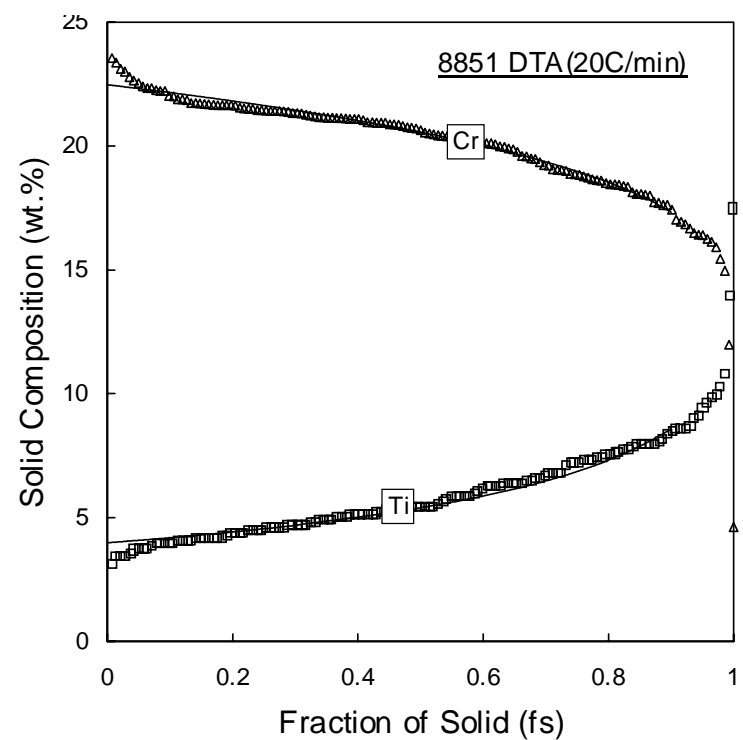

(b)

Figure 5-3: Solid composition profiles in model alloys (a) HV8851, (b) HV8852, and (c) HV8853. 


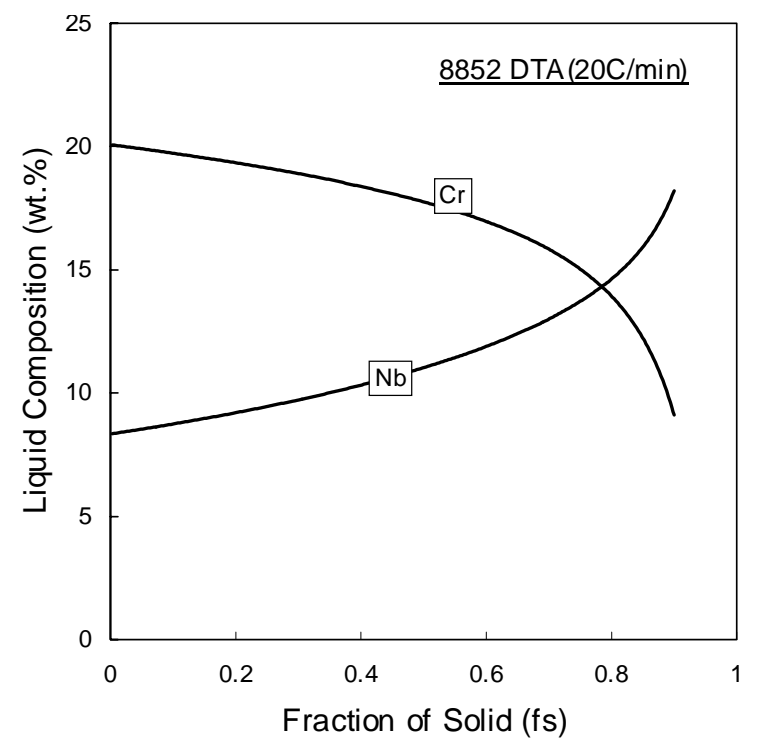

(a)

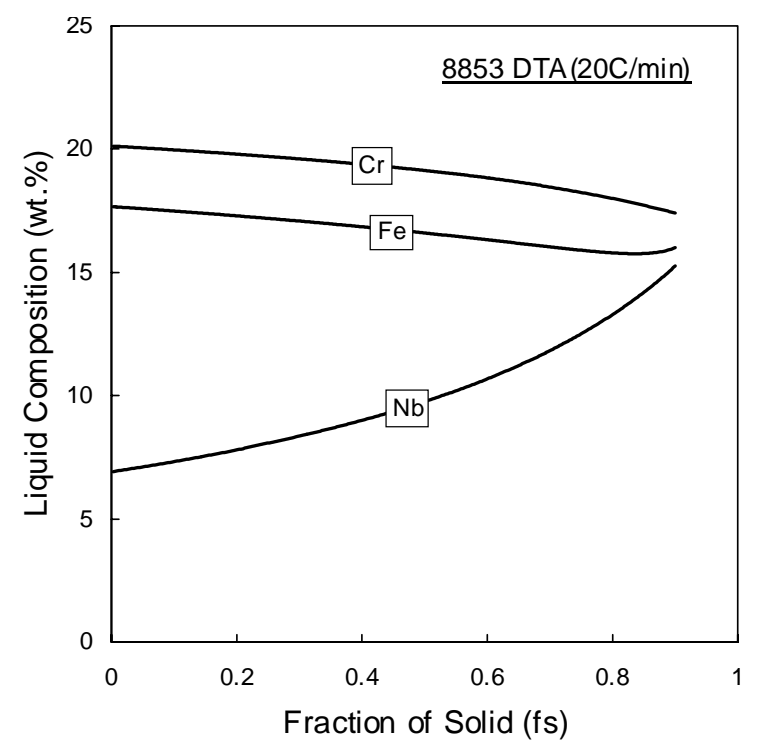

(c)

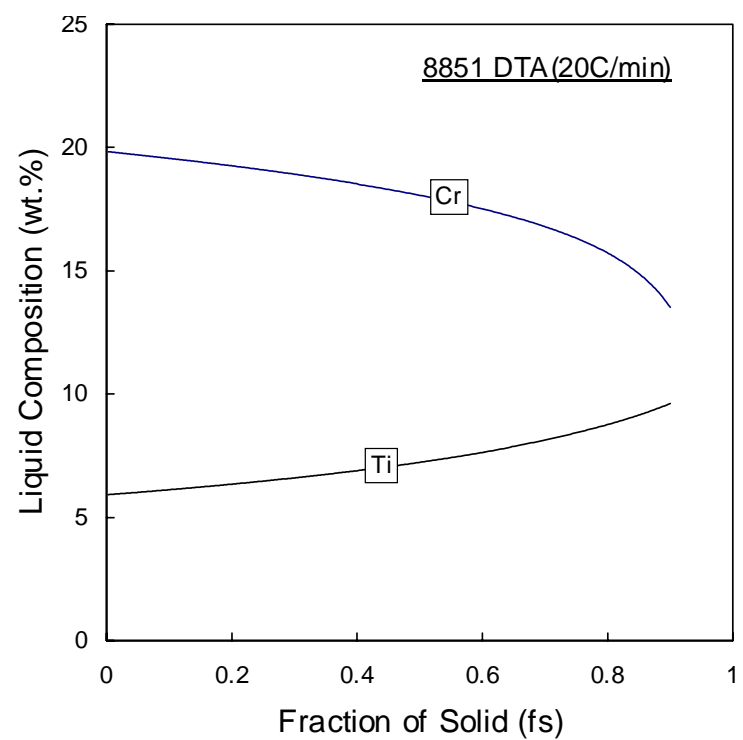

(b)

Figure 5-4: Liquid composition profiles in model alloys (a) HV8851, (b) HV8852, and (c) HV8853. 


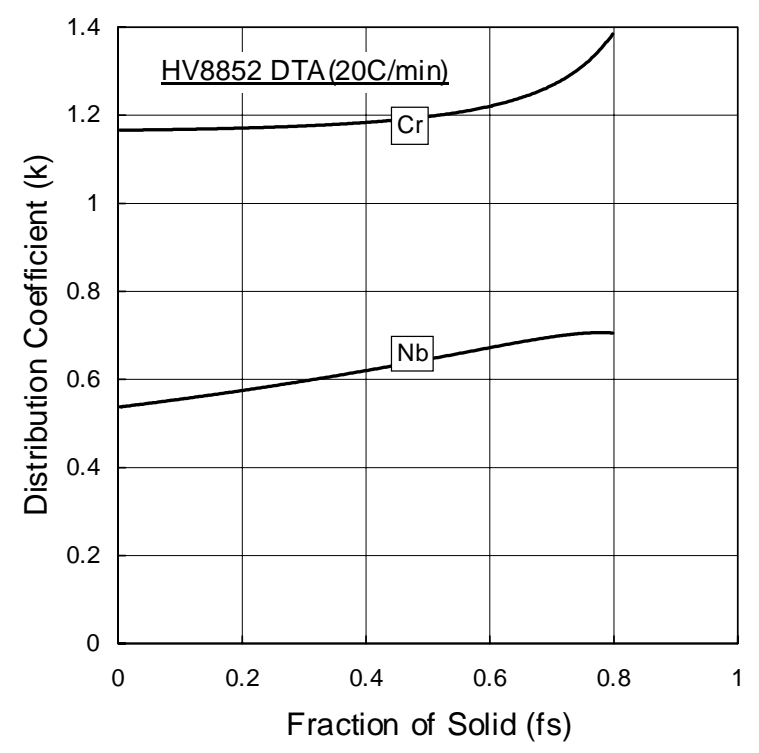

(a)

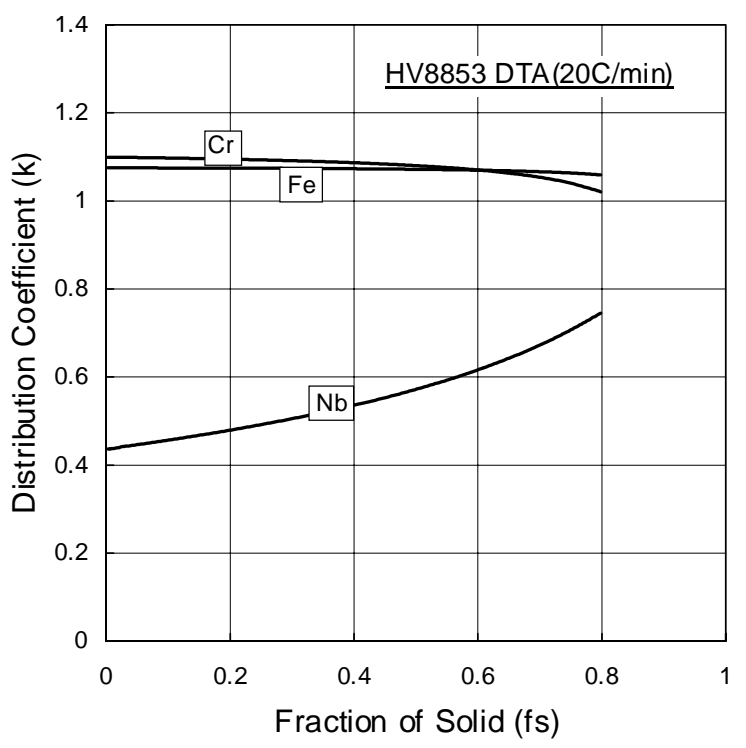

(c)

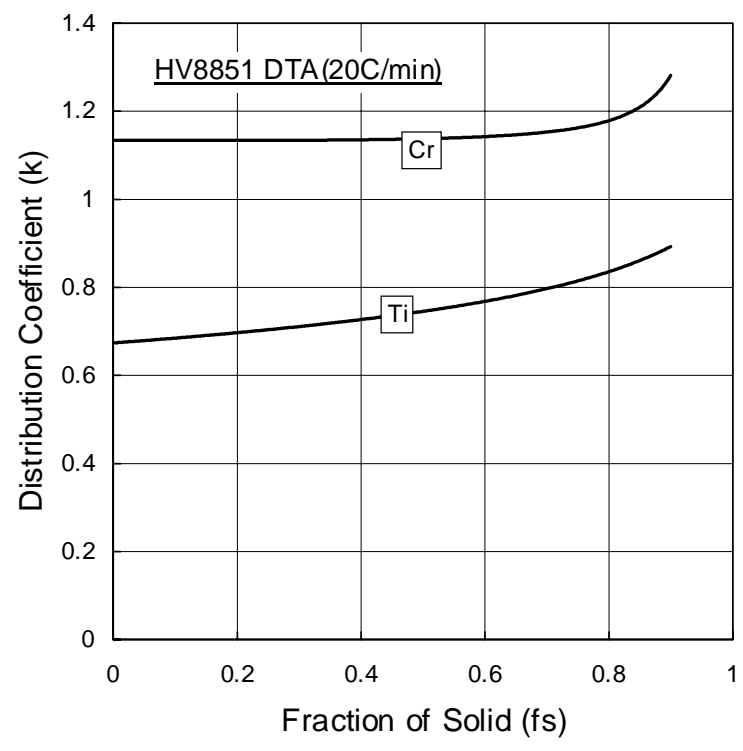

(b)

Figure 5-5: Elemental partition ratio profiles in model alloys (a) HV8851, (b) HV8852, and (c) HV8853. 


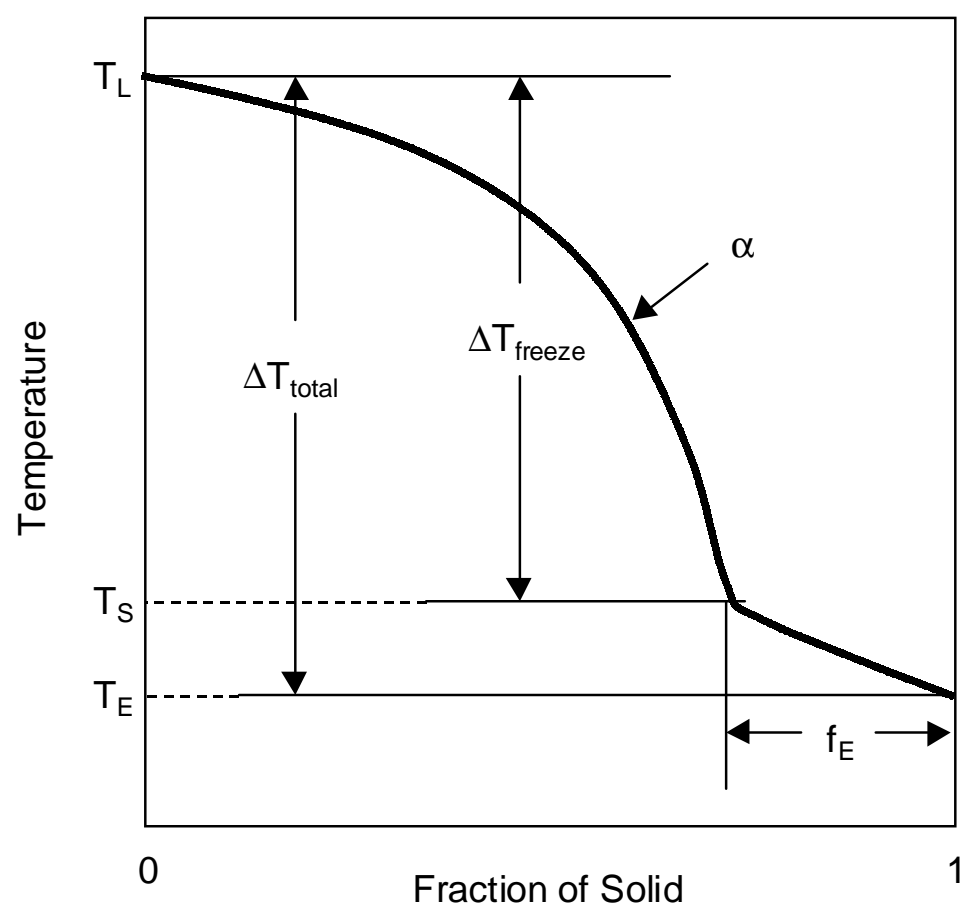

Figure 5-6: Characteristic features of $f_{\mathrm{s}}(T)$ curve in superalloys.
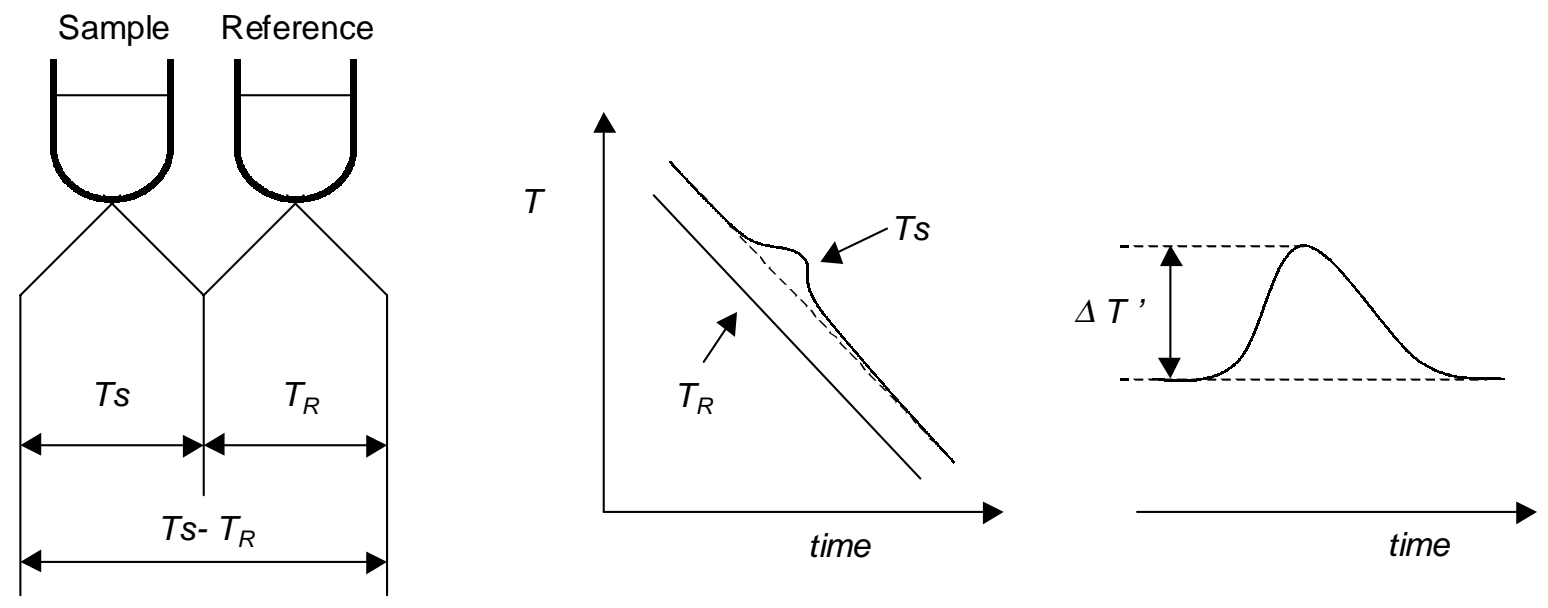

Figure 5-7: Principle of quantitative DTA. 

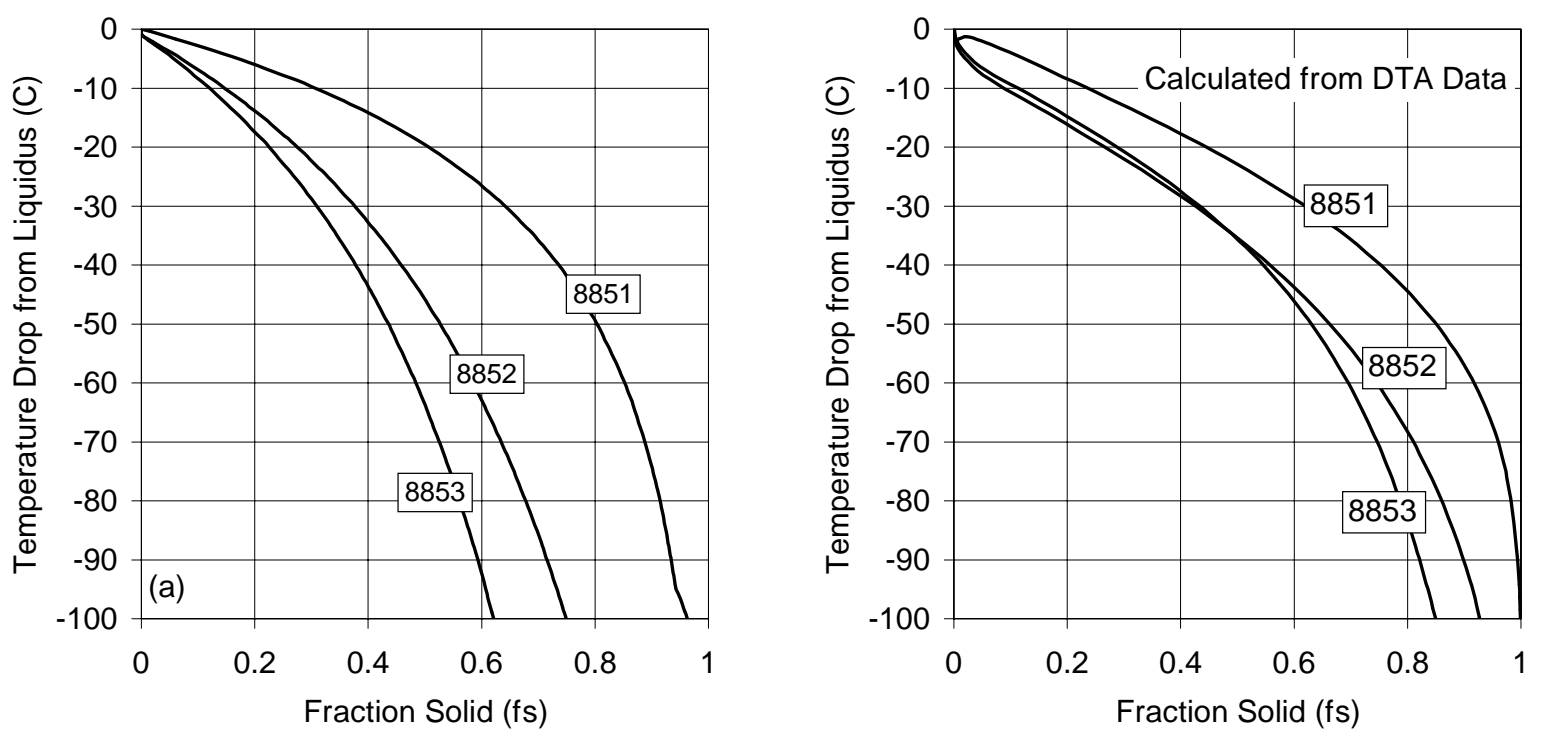

Figure 5-8: $f \mathrm{~s}(T)$ curves of HV8851, HV8852 and HV8853 by Thermo-Calc (left) and quantitative DTA analysis (right).
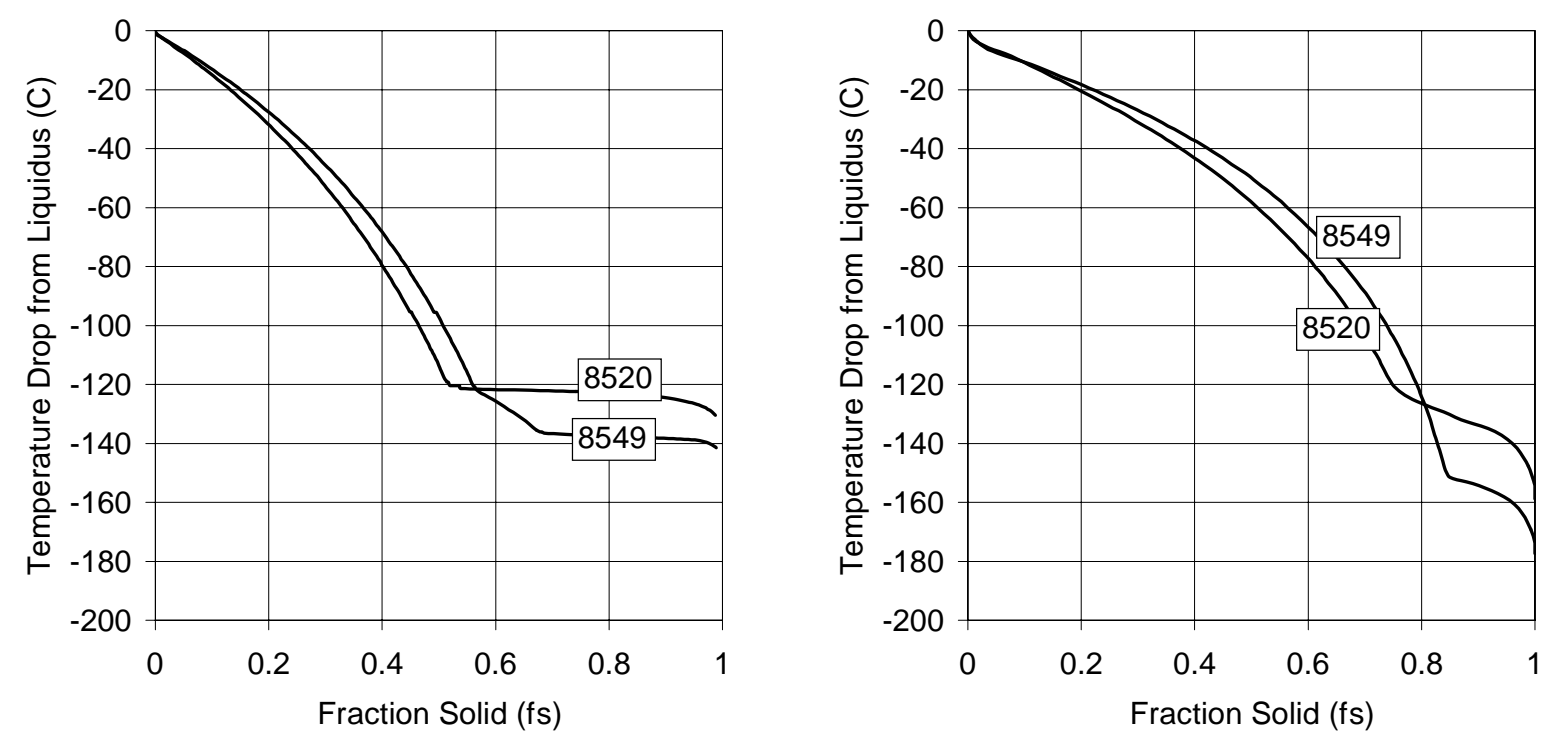

Figure 5-9: $f \mathrm{~s}(T)$ curves of HV8520 and HV8549 by Thermo-Calc (left) and quantitative DTA analysis (right). 

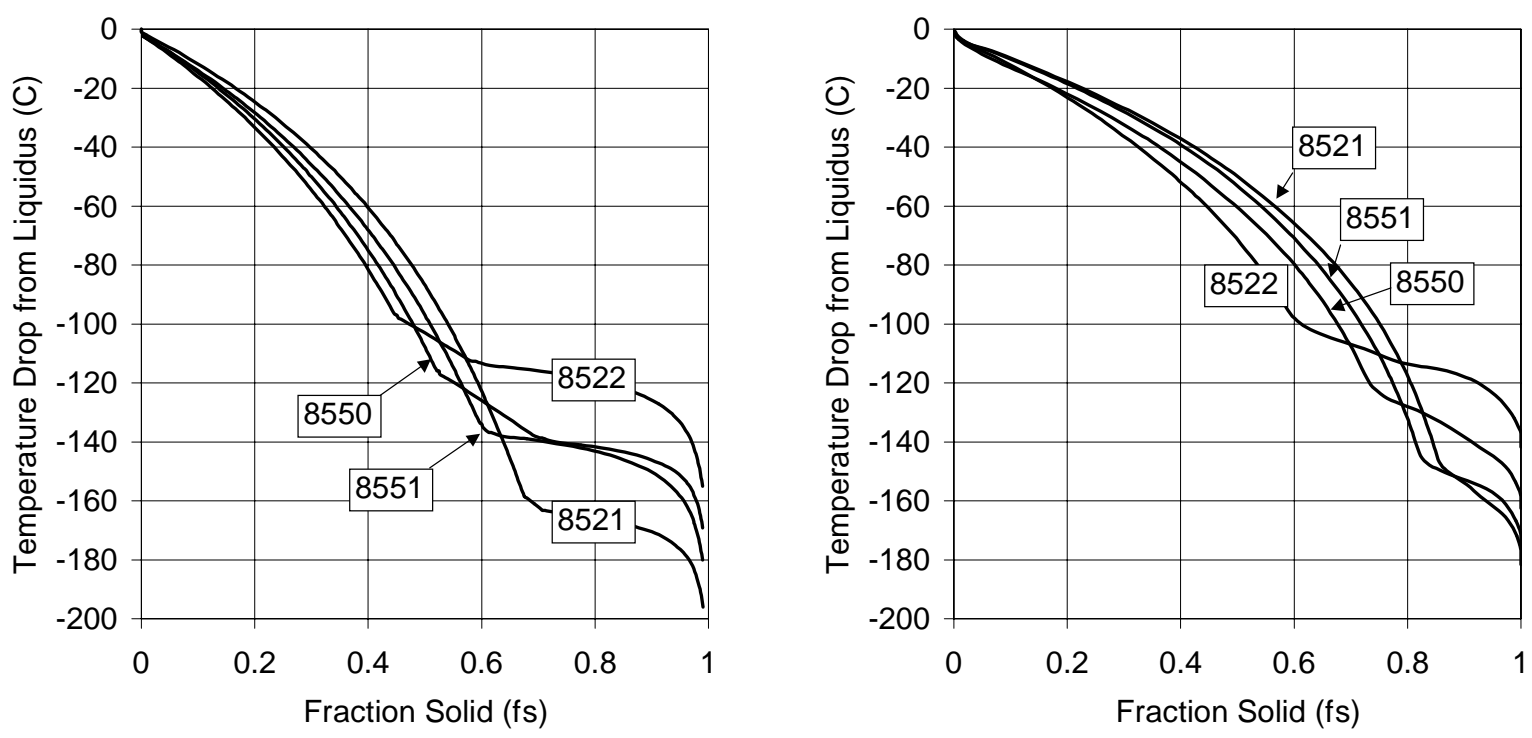

Figure 5-10: $f_{\mathrm{s}}(T)$ curves of HV8551, HV8550, HV8520 and HV8521 by Thermo-Calc (left) and quantitative DTA analysis (right).
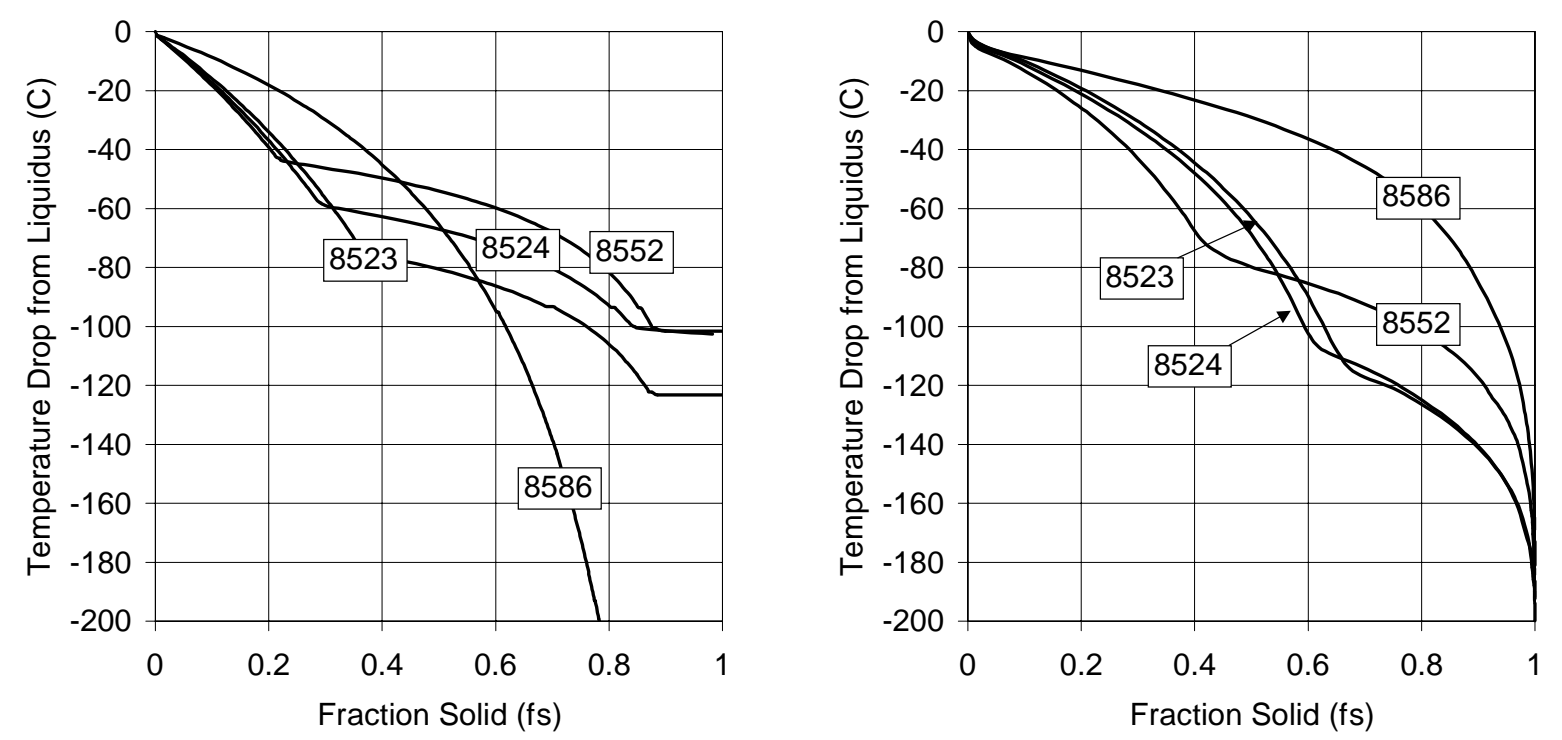

Figure 5-11: $f \mathrm{~s}(T)$ curves of HV8523, HV8524, HV8552 and HV8586 by Thermo-Calc (left) and quantitative DTA analysis (right). 

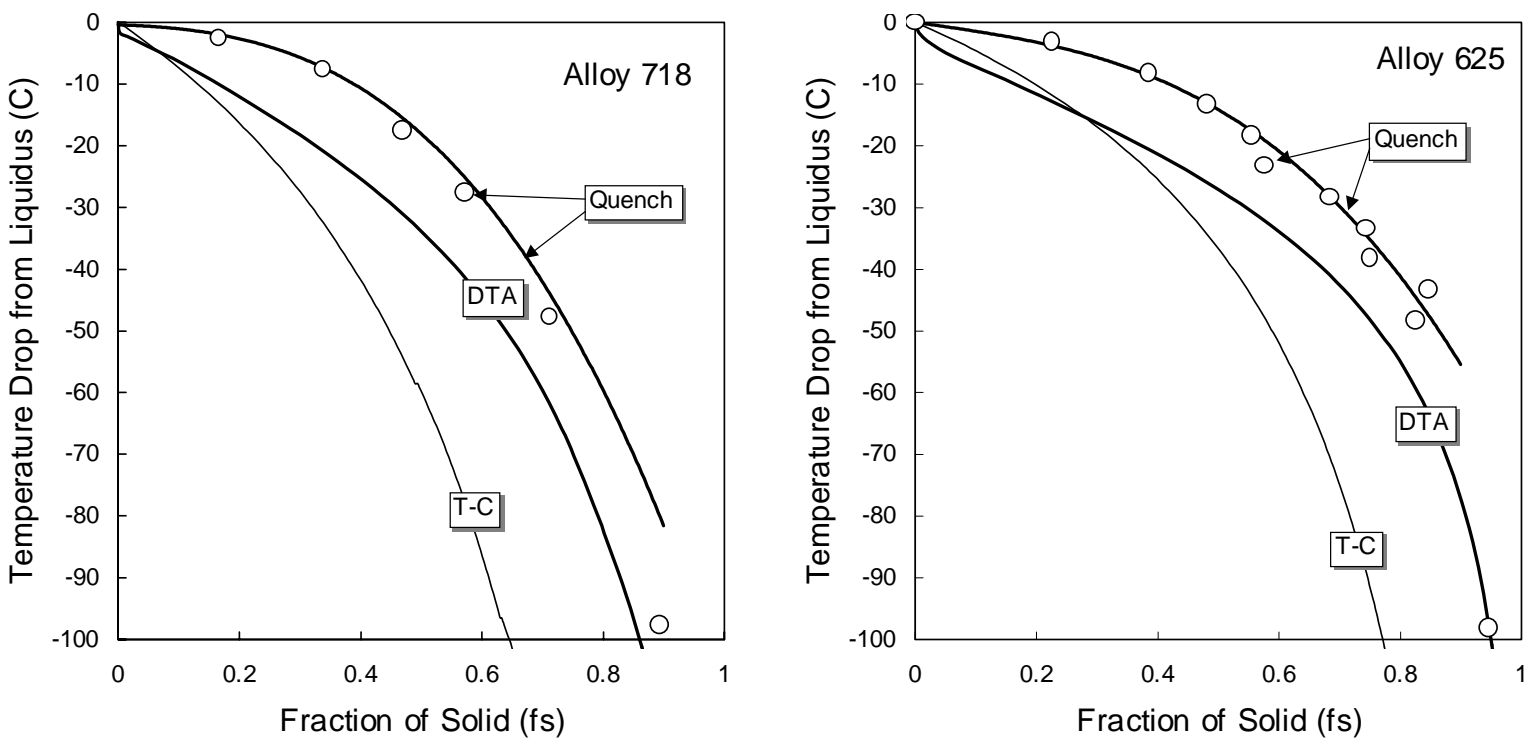

Figure 5-12: Comparison of quantitative DTA with quenching results in alloys 718 and 625.

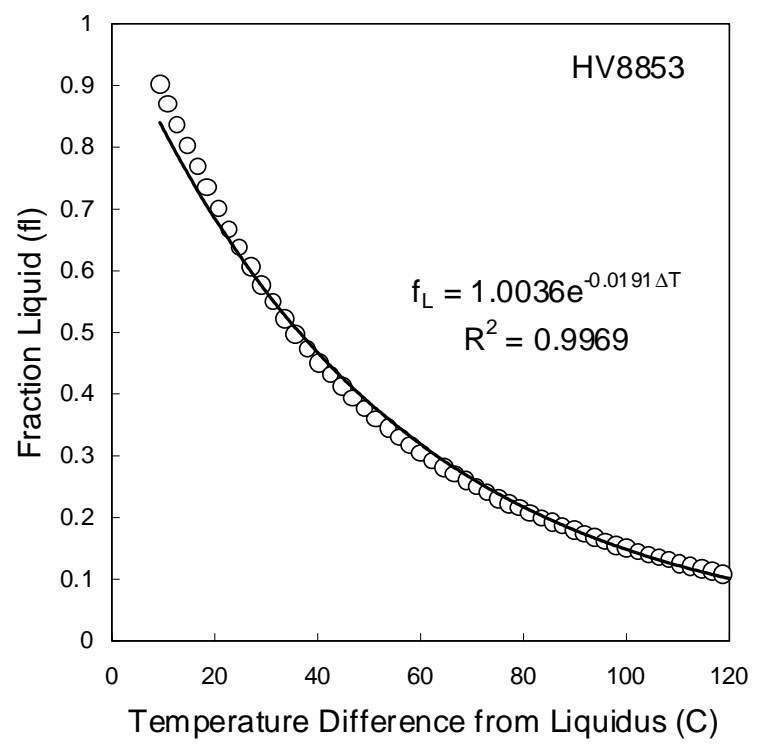

Figure 5-13: Fitting $\mathrm{f}_{\mathrm{L}}(\Delta \mathrm{T})$ curve using exponential function. 

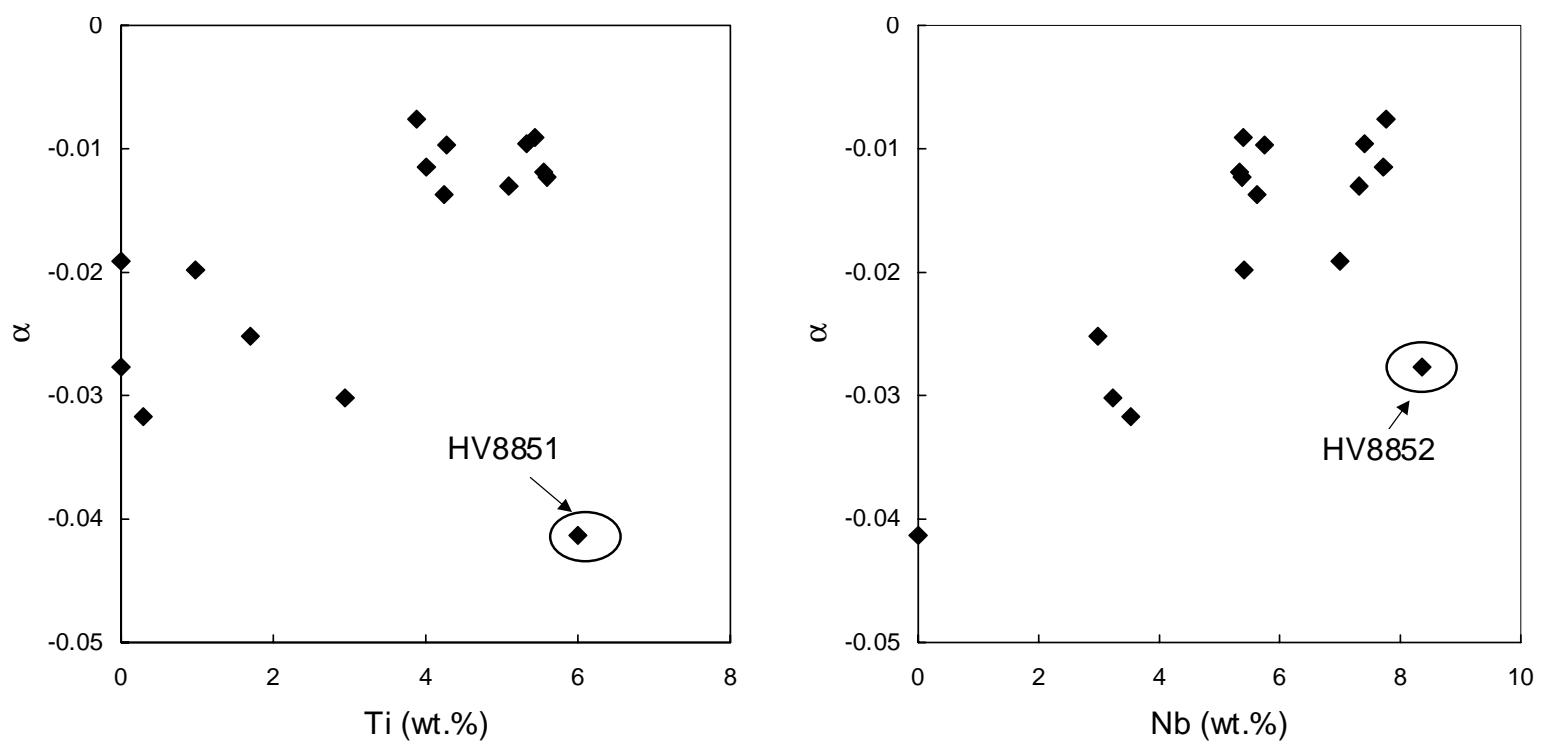

Figure 5-14: Correlations of $\alpha$ with $\mathrm{Ti}$ (left) and $\mathrm{Nb}$ (right) concentrations. Circled data points (ternary alloys HV8851 and HV8852) are outliers and excluded from regression.

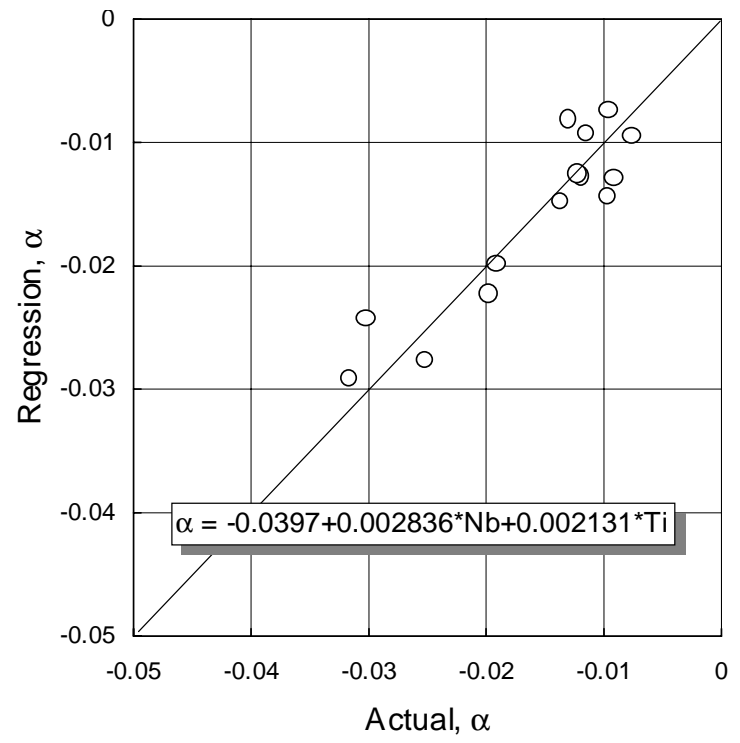

Figure 5-15: Comparison of predicted and actual $\alpha$ values in superalloys. 


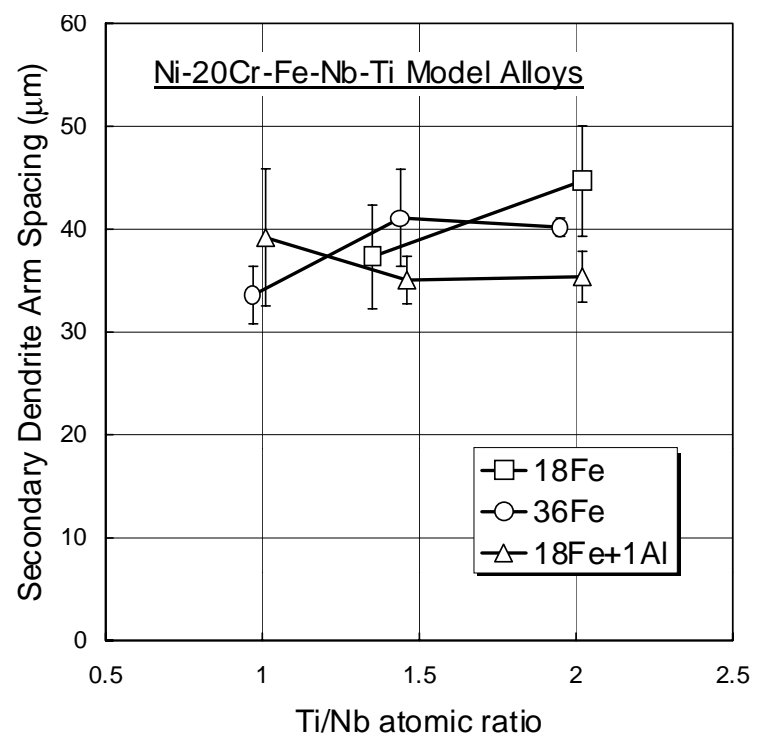

(a)

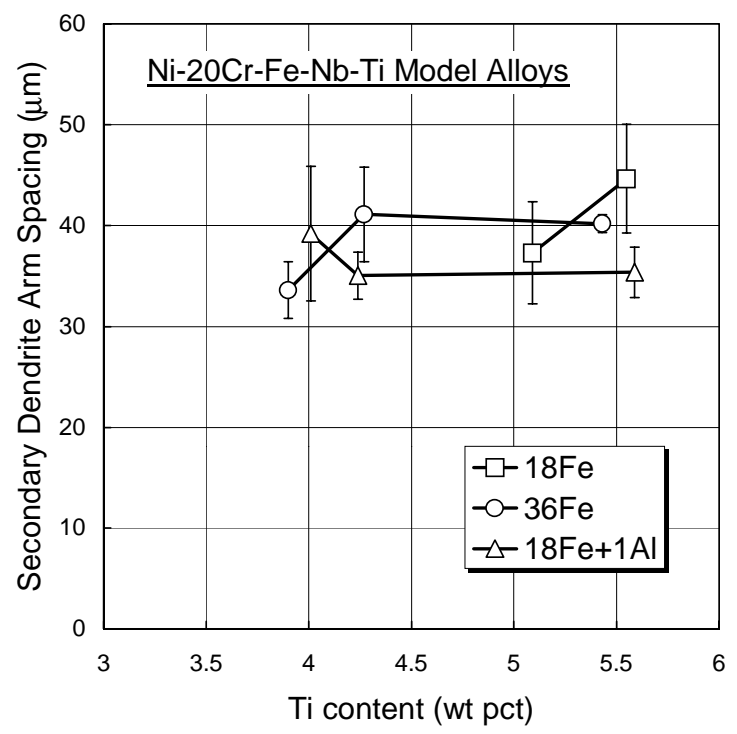

(b)

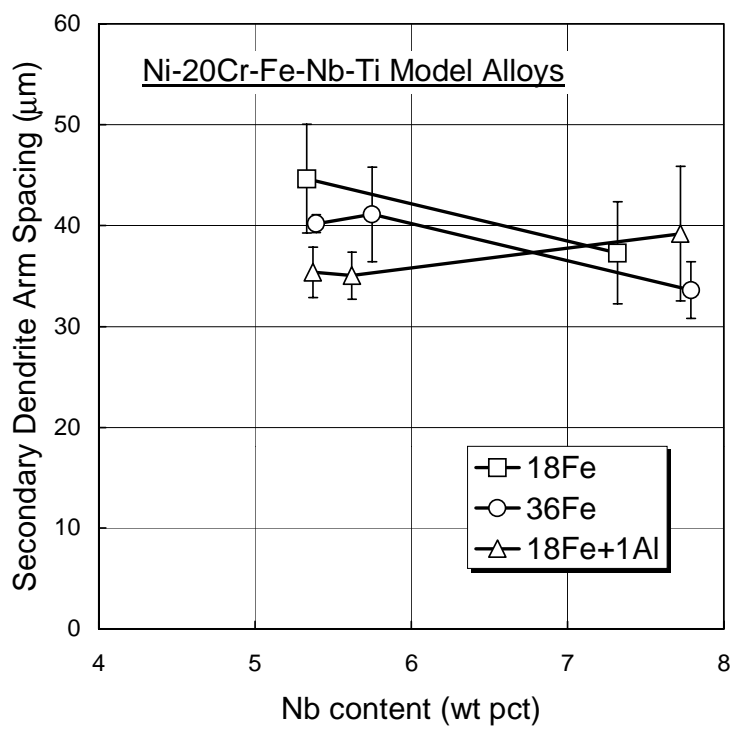

(c)

Figure 5-16: Secondary dendrite arm spacings versus (a) Ti/Nb atomic ratio, (b) Ti content, and (c) $\mathrm{Nb}$ content in model alloys. 
Chapter 6

\section{Freckling Criteria}

6.1. Introduction 115

6.2. Rayleigh Criteria 115

6.3. Sensitivity of Key Control Parameters 118

$\begin{array}{ll}\text { 6.3.1. Viscosity } & 118\end{array}$

$\begin{array}{ll}\text { 6.3.2. Permeability } & 118\end{array}$

6.3.3. Liquid Density 119

$\begin{array}{ll}\text { 6.3.4. Fraction of Solid } & 120\end{array}$

6.4. Composition Effects 121

6.4.1. Driving Force 121

6.4.2. Resistance 122

6.5. Freckling Tendencies in Model Alloys 124

6.5.1. Relative Rayleigh Number 124

6.5.2. Freckling Tendencies in Model Alloys 124

6.6. Freckling in Industrial Alloys 126

6.7. Discussion 126

6.7.1. Processing Conditions 126

$\begin{array}{lll}\text { 6.7.2. } & \text { Ingot Size } & 127\end{array}$

$\begin{array}{lll}\text { 6.8. Summary } & 127\end{array}$ 


\subsection{Introduction}

Several criteria have been developed based on density inversion theory in attempt to explain freckling phenomena in directional solidification (see summary in chapter 1). However, none of them offers a satisfying solution for freckling problem in remelt processed superalloys where heavier interdendritic liquid and sloped solidification interface are present. Addition of multiple solute elements makes it increasingly difficult to produce large diameter ingots that are free of freckles. It is vitally important to have a process model that is capable to consider both alloying and processing effects.

The main purpose of this chapter is to develop a freckling model based on instability of interdendritic liquid flow under remelt conditions. The horizontal component in remelt ingot has the highest propensity to freckle formation and can be correctly simulated using the horizontal solidification experiment. Key control parameters identified under horizontal solidification conditions are incorporated into the criterion and a sensitivity study is given to account for various sources of error encountered during the measurement and analysis of these parameters. Composition effects on the driving force and resistance for interdendritic liquid flow are discussed in detail. Based on the analysis, a composition-related freckling criterion, e.g. the relative Rayleigh number, is proposed. The proposed criterion is then used to analyze the freckling tendencies of model alloys HV8851 (Ni-20Cr-6Ti), HV8852 (Ni-20Cr-8.6Nb), and HV8853 (Ni-20Cr-18Fe-7Nb) that have been designed for the physical simulation of freckling. Effects of process conditions, such as the pool geometry and ingot size, are also discussed.

\subsection{Rayleigh Criterion}

A solute redistribution model for a small volume element inside the mushy zone is shown in Figure 6-1. Solute enters or leaves the element only by fluid flow and mass flow in or out of the element by diffusion is neglected. Solute redistribution within the volume element can be described by the following equation after Flemings' analysis [13] (please refer to Appendix A for derivation of equation (6-1)): 


$$
\frac{\partial f_{L}}{\partial C_{L}}=-\left(\frac{1-\beta}{1-k}\right)\left[1+\frac{\vec{V} \cdot \nabla T}{\varepsilon}\right] \frac{f_{L}}{C_{L}}
$$

Where $f_{L}$ is the volume fraction of liquid, $C_{L}$ is the liquid composition, $\beta$ is the solidification shrinkage, $k$ is the equilibrium partition ratio, $\vec{V}$ is the vector form of interdendritic fluid flow velocity, $\varepsilon$ is the cooling rate. The equation reduces to Scheil condition when the flow velocity becomes zero and solidification shrinkage is neglected as shown in Figure 6-1.

Multiplying both sides of equation (6-1) leads to:

$$
\frac{\partial f_{L}}{\partial T}=-\left[\frac{1}{1-k} \cdot \frac{f_{L}}{C_{L}} \cdot \frac{\partial C_{L}}{\partial T}\right]\left(1+\frac{\vec{V} \cdot \nabla T}{\varepsilon}\right)
$$

The quantity inside the first pair of brackets is always negative regardless of the value of $k$. The term on the left of eq. (6-2) becomes negative if the following condition is reached:

$$
\frac{\vec{V} \cdot \nabla T}{\varepsilon}<-1
$$

That is, the fraction of liquid in the volume element increases with the decrease of temperature. Interdendritic liquid flow becomes unstable and the perturbed channel grows as remelting occurs in the volume element until it becomes visible as freckle segregate. Figure 6-2 is a schematic illustration of stable and unstable liquid flow under both vertical DS and sloped remelting conditions. In both configurations,

$$
\varepsilon=-\nabla T \cdot \vec{R}
$$

$\vec{R}$ is the vector form of the crystal growth rate. Combining (6-3) and (6-4), the freckling criterion of (6-3) becomes:

$$
\frac{\vec{V} \cdot \vec{n}}{\vec{R} \cdot \vec{n}}>1
$$

In equation (6-5), $\vec{n}$ is the vector of crystal growth direction. Here vector forms are used because the direction of maximum liquid flow may be different from the crystal growth direction in remelting. It is noted that $\vec{R} \cdot \vec{n}$ is the local crystal growth velocity of columnar grains and may not be equal to the overall solidification speed of the ingot. The physical meaning of equation (6-5) is that if the component of interdendritic flow velocity 
along the direction of crystal growth is greater than the crystal growth rate, freckles will form as a result of unstable fluid flow.

The interdendritic flow velocity through the volume element is linearly related to the pressure gradient due to density difference and D'Arcy's law applies:

$$
\vec{V}=-\frac{\Delta \rho \cdot g \cdot \Pi}{\eta \cdot f_{L}} \cdot F(\theta, x, y)
$$

Where $\eta$ is the viscosity of interdendritic fluid, $\Pi$ is the permeability, $g$ is acceleration due to gravity, $\Delta \rho$ is the density difference inside the mushy zone. $F(\theta, x, y)$ is the pool geometry factor including effects of solidification front angle $(\theta$, the angle between the longitudinal axis of columnar grains and the horizontal axis) and the $(x, y)$ coordinates of the location of interest in the mushy zone. Definitions of the coordinate system and the effect of pool geometry factor will be discussed in section 6.5.1. The channel flow velocity is determined by the driving force due to density gradient as well as the resistance of the dendritic network.

Substituting (6-6) into (6-5) leads to the explicit freckling criterion:

$$
F(\theta, x, y) \cdot \frac{\Delta \rho \cdot g \cdot \Pi}{\eta \cdot f_{L}} \cdot \frac{\vec{n}}{\vec{R} \cdot \vec{n}}>1
$$

Since $\vec{R}$ is the ingot growth rate with vertically upward direction, $\vec{R} \cdot \vec{n}$ can be replaced by $R \cdot \sin \theta$, where $\mathrm{R}$ is the nominal ingot growth rate. In remelt process, the angle between freckle channel and crystal growth direction is not readily known, hence, equation (6-7) can be re-written as:

$$
R a=F(\theta, x, y) \cdot \frac{\Delta \rho g \Pi}{\eta f_{L}} \cdot \frac{1}{R \sin \theta}>R c
$$

$R a$ is Rayleigh number and $R c$ is the critical Rayleigh number for freckle formation. Here the concept of Rayleigh number is used because equation (6-8) described the situation that unstable convection occurs when the buoyancy contribution exceeds the restraining pressure, which is physically similar to the formulations of Rayleigh numbers by Sarazin and Hellawell [22]. The reason why various forms of Rayleigh number are present is that the nature of the liquid convection is not fully understood. The Rayleigh numbers described by equations (1-6) and (1-7) treat the dissipation of heat or mass to the surrounding environment to be the limiting factor for freckle flow. If the rate of 
dissipation is fast enough, no continuous flow can be sustained. Hence, the thermal diffusivity or the liquid diffusion coefficient appears in the denominator. However, equation (6-5) describes the situation where the competition between the interdendritic flow and the crystal growth dominates the convection pattern. Freckles will form if the interdendritic flow outpaces the local crystal growth. Thus, the local crystal growth rate becomes the limiting factor for channel flow. This is of practical importance since it relates the processing conditions (overall ingot growth rate) with the freckling criterion.

In the current study, horizontal solidification has been used as the physical simulation of freckling. It correctly simulates the horizontal component in remelting ingots where the freckling tendency is the highest. It is noted that the freckling model in equation (6-8) incorporates all of the key control parameters that have been identified and investigated in previous chapters. Freckling potentials of model alloys will be compared with experimental result from horizontal solidification in section 6.5. Therefore the freckling criterion is capable to explain the freckling phenomena in remelt superalloys.

\subsection{Sensitivity of Key Control Parameters}

The sensitivity of the freckling criterion depends on those of the key control parameters, which can be obtained via either experiments or numerical evaluation. The sensitivities of the experimental equipment and numerical methods are discussed in this section.

\subsubsection{Viscosity}

Viscosity of liquid can be numerically evaluated for any alloy composition at any temperature through the following expression published in references $[50,51]$ :

$$
\begin{gathered}
\eta=A \cdot \exp (B / R T) \\
A=\frac{1.7 \times 10^{-7} \cdot \rho^{2 / 3} \cdot T_{L}^{0.5} \cdot M^{-1 / 6}}{\exp \left(B / R T_{L}\right)} \\
B=2.65 \cdot T_{L}^{1.27} \\
R=8.3144 \quad \mathrm{~J} / \mathrm{mol} \cdot K
\end{gathered}
$$

It is assumed that viscosity does not vary significantly during solidification from alloy to alloy and is only dependent on temperature. 


\subsubsection{Permeability}

The liquid flow in remelting is mainly parallel to the slope of mushy zone, e.g. normal to the primary dendrite arms. The permeability depends upon the secondary dendrite arm spacing $\left(d_{2}\right)$ as well as $d_{1}$ and $f_{\mathrm{L}}$ and is expressed by the following [52]:

$$
\Pi_{1}=9.66 \times 10^{-18} d_{1}^{0.699} d_{2}^{2.73} f_{L}^{3.34} \quad( \pm 31 \%)
$$

The permeability is in $\mathrm{m}^{2}$. Dendrite arm spacings are in $\mu \mathrm{m}$. The permeability for flow that is parallel to primary dendrite arms is given by:

$$
\Pi_{2}=3.75 \times 10^{-4} d_{1}^{2} f_{L}^{2} \quad( \pm 30 \%)
$$

Here dendrite arm spacing is in $\mathrm{m}$. Auburtin [26] has evaluated the permeability of VAR/ESR ingots. It has been observed that flow perpendicular to the direction of primary dendrites should be easier than that parallel to the dendrites due to a permeability of 2.5 times higher.

\subsubsection{Liquid Density}

Direct measurement of liquid density of Ni-based superalloys is difficult because of the high melting temperature and reactive nature of the alloy. Liquid density can be expressed using the following equation:

$$
\rho=\rho_{0} \cdot\left[1+\sum_{i} \beta_{i} \Delta C_{i}+\beta_{T} \Delta T\right]
$$

Thus the accuracy of density calculation depends on the type of equipment used to measure composition and reaction temperatures.

\subsubsection{Composition Measurement - EDS}

In the current study, EDS is used as the primary tool to measure elemental compositions. To determine the random fluctuation caused by the equipment, a homogenized alloy 625 sample was used. A $10 \mu \mathrm{m}$ by $10 \mu \mathrm{m}$ area on the sample surface was covered by a virtual 9x9 grid and composition at each grid point was measured using EDS. Because the sample has been homogenized and the sampling area is very small, variation in the group of data represents the random fluctuation of the equipment only. Standard deviations of each alloying element are used for regression analysis to generate a relation between the standard deviation and the nominal alloy compositions. The equation is written: 


$$
\text { STDEV }=0.0106 \cdot[C]+0.2387
$$

The R square of regression analysis is $0.9526,[\mathrm{C}]$ is the nominal composition in wt pet. Equation (6-14) indicates the dependence of standard deviation on alloy composition, which shows that the standard deviation increases with the alloy composition. For example, for $55 \mathrm{wt}$ pct of $\mathrm{Ni}$, the standard deviation of measurement would be $0.82 \mathrm{wt}$ pct.

\subsubsection{Reaction Temperature - DTA}

The TA-1600 DTA cell used in the current study has been calibrated at both higher and lower bounds of interested reaction temperatures by pure nickel and silver standards. The accuracy of calibration is within $\pm 2^{\circ} \mathrm{C}$. As discussed previously in section 5.5, other factors may also contribute to the ability of DTA to determine characteristic temperatures of complex alloys. These factors include the undercooling, chemistry effect (especially the trace elements), and the sensitivity of the equipment in discerning adjacent reaction temperatures.

\subsubsection{Liquid Density Calculation - "Metals"}

Basic theoretical treatment of density calculation has been given previously in chapter 2 . "Metals", a computer program compiled and provided by National Physics Lab in UK, is adopted and improved to calculate liquid density in the current research. The model is held to be accurate to about 5\%. After the mixing effect is corrected, the calculation error is reduced to less than $3 \%$ as compared with experimental liquid densities of superalloys.

\subsubsection{Fraction of Solid}

Fraction of solid, $f_{\mathrm{S}}$, can be determined using the quantitative DTA technique described in chapter 5. The accuracy of the method depends on the equipment of DTA and the model itself such that other forms of heat loss may be included. It is assumed that at the temperature of interest of superalloys, radiation heat transfer is dominant and no data is available to quantify the effects of other heat loss modes. Since it is of more importance to compare the evolution of $f \mathrm{~s}$ between alloys than to obtain an absolute value and the relative order of results is in good agreement with that determined by Thermo-Calc, accuracy of the approach is only related to the DTA equipment $\left( \pm 2^{\circ} \mathrm{C}\right)$. 
Other methods, such as interrupt quenching combined with image analysis and regression analysis of liquidus as described in reference [53], also employ DTA as the major experimental technique. Image analysis can be realized using automated software or manual counting of the quenched solid (or liquid) areas using available photographs, both of which could introduce bias. The undercooling of the interrupt quenching is estimated to be $5^{\circ} \mathrm{C}$ and needs to be corrected for the temperature data. Regression analysis of liquidus of series of Ni-based alloys was described in detail in reference [53]. The analysis yielded a linear correlation between the liquidus temperature and the elemental concentrations in the liquid. The results can give relatively accurate prediction of liquidus of alloys with dilute solute concentrations. The prediction may deviate from reality due to higher-order interactions of the elements when they are significantly enriched in the liquid during solidification.

\subsection{Composition Effects}

From eq. (6-8), the Rayleigh criterion can be separated into two-folds as (1) Composition related: $\Delta \rho \Pi / f_{L}$ and (2) Processing condition related: $1 / R \sin \theta$. The solidification front angle $(\theta)$ can be affected by composition, but more significantly by processing conditions.

\subsubsection{Driving Force}

The driving force for freckle formation is provided by liquid density difference in the mushy region. Equation (6-13) can be transformed by substituting Scheil equation:

$$
\frac{\Delta \rho}{\rho_{0}}=\sum \beta_{i} C_{i}^{0}\left[\left(1-f_{S}\right)^{k-1}-1\right]+\beta_{T}\left(T-T_{0}\right)=\frac{1}{\rho_{0}}\left(\Delta \rho_{C}+\Delta \rho_{T}\right)
$$

It is clearly shown in equation (6-14) that density difference is affected by both solute redistribution and temperature change. The total density change can be broken down as $\Delta \rho_{C}$, the solutal density change, and $\Delta \rho_{T}$, the thermal density change.

The solutal density difference depends on the nominal alloy composition $\left(C_{i}^{0}\right)$, the partition ratio $(k)$ and the solutal expansion coefficient $\beta_{i}$ of each element. Alloying elements with smaller partition ratio and higher nominal concentration such as $\mathrm{Nb}$ and $\mathrm{Ti}$ 
would have larger impacts on the density difference. Thermal effect can be explained as that density changes with temperature even if there is no composition difference in liquid. The effect of temperature difference depends on the freezing ranges (equations (4-5) and (4-6)) as well as the thermal expansion coefficient $\beta_{T}$. The larger the freezing range, the greater the temperature effect. Based on previous discussion (chapter 4), for dilute alloys freezing range increases with solute concentrations and decreases if the concentration exceeds solubility. Effect of $\beta_{T}$ is discussed in the following paragraph.

Table 6-1: The breakdown of liquid density changes in model alloys

\begin{tabular}{lcccccc}
\hline Alloys & $\mathbf{6 T i}$ & $\mathbf{8 . 6 N b}$ & $\mathbf{1 8 F e - 7 N b}$ & $\mathbf{7 1 8}$ & $\mathbf{7 0 6}$ & $\mathbf{6 2 5}$ \\
\hline Fraction Solid $(\mathrm{fs})$ & 0.4 & 0.4 & 0.4 & 0.4 & 0.4 & 0.4 \\
Solutal $\Delta \rho_{C}\left(\mathrm{~kg} / \mathrm{m}^{3}\right)$ & -39 & 118 & 50 & 32.97 & -21.15 & 27.05 \\
Thermal $\Delta \rho_{T}\left(\mathrm{~kg} / \mathrm{m}^{3}\right)$ & 13 & 20 & 28 & 26.5 & 21.29 & 16.26 \\
Total $\Delta \rho\left(\mathrm{kg} / \mathrm{m}^{3}\right)$ & -26 & 138 & 78 & 59.47 & 0.14 & 43.31 \\
$\Delta \mathrm{T}\left({ }^{\circ} \mathrm{C}\right)$ & -12.32 & -18.56 & -26.9 & -25.71 & -20.2 & -16.06 \\
$d \rho / d T\left(\mathrm{~kg} / \mathrm{m}^{3} /{ }^{\circ} \mathrm{C}\right)$ & -1.055 & -1.078 & -1.041 & -1.031 & -1.054 & -1.012 \\
\hline
\end{tabular}

Note: $6 \mathrm{Ti}=\mathrm{Ni}-20 \mathrm{Cr}-6 \mathrm{Ti}, 8.6 \mathrm{Nb}=\mathrm{Ni}-20 \mathrm{Cr}-8.6 \mathrm{Nb}, 18 \mathrm{Fe}-7 \mathrm{Nb}=\mathrm{Ni}-20 \mathrm{Cr}-18 \mathrm{Fe}-7 \mathrm{Nb}$.

The individual contribution of the solutal and thermal effects is worth of attention. Table 6-1 is the breakdown of the liquid density changes. For alloy HV8852, 15\% of the density change comes from thermal effect, while it increases to $36 \%$ in alloy HV8853. In HV8851, the thermal effect is even greater. The large thermal density change is due to the nature of transition metals, e.g. $\mathrm{Ni}, \mathrm{Cr}, \mathrm{Nb}$, etc. The temperature coefficient of liquid density, $d \rho / d T$, is $-1.159 \mathrm{~kg} / \mathrm{m}^{3} /{ }^{\circ} \mathrm{C}$ for pure $\mathrm{Ni}$. This value is comparable with those obtained for model alloys and industrial alloys in Table 6-1 since $\mathrm{Ni}$ is the major constituent of the alloys. For alloy 706, the absolute value of solutal density difference is almost the same as the thermal density difference. The significant thermal contribution to the change in liquid density is the intrinsic nature of Ni-based alloys.

\subsubsection{Resistance}

The fluidity resistance is affected by the permeability in two folds. First, low permeability in dendritic region will shift the freckle initiation site to a lower fraction of solid. Some 
people defined this place as the solidification front. Accordingly the driving force to float up the solute enriched liquid does not become large enough to form freckles since the solute is not sufficiently enriched in the interdendritic liquid at the solidification front. Second, in some cases some local channel flow will persist in the mushy zone. However, these channels will be dissolved or become discontinuous if the permeability is low. From regression analysis of $\mathrm{Pb}-\mathrm{Sn}$ alloys [Poirier 1989], the liquid permeability normal to primary dendrites depends on both primary and secondary dendrite arm spacings and fraction of solid (equation 6-10). It is noted that these attributes are only the measurable characteristics of dendrite morphology. There are other features that cannot be easily obtained may also influence the permeability. The characteristic morphology of dendrites that contribute to a low permeability (vise versa) is described as follows:

1) Smaller dendrite arm spacing, including $d_{1}$ and $d_{2}$.

2) Higher fraction of solid.

3) Smaller curvature at the tip of dendrite.

4) Higher order dendrites tend to form easily.

Character 1) is probably the most important factor that influences permeability. It has been frequently related to processing conditions such as $G$ and $R$. However, almost no data is available for composition effects on dendrite spacings. The composition effect on spacings needs more thorough investigation. Effect of fraction solid in character 2) can be illustrated by equation (5-4), in which $\alpha$ describes the absolute value and the shape of $f_{\mathrm{S}}(T)$. Alloy with higher levels of $\mathrm{Nb}$ and $\mathrm{Ti}$ has higher $\alpha$, i.e. lower fraction solid in the mushy zone at the same temperature drop. $\mathrm{Nb}$ has more pronounced effect than Ti. Character 3) means that dendrites are easily connected with each other because of the high growth rate of dendrite tips. Character 4) means that the space for flowing liquid, i.e. flowing channel, is narrow. Future study is needed for alloying effect on 3) and 4).

\subsection{Freckling Tendency of Model Alloys}

\subsubsection{Relative Rayleigh Number}

In industry, remelt ingots for different superalloys are often produced with comparable size and geometry. It can be simplified that the pool geometry and processing conditions are 
similar for various alloys provided the processes and ingot size are the same. A composition-related freckling index, $R a^{*}$, is proposed to address the composition effects on freckling tendency. Since it is derived from Rayleigh number by excluding the processing effect, it is called the relative Rayleigh number and can be defined as follows:

$$
R a^{*}=\frac{\Delta \rho \Pi}{f_{L}}>R c^{*}
$$

$R c^{*}$ is the critical number for freckle formation. It is noted that the constant and the dendrite arm spacings $\left(d_{1}\right.$ and $\left.d_{2}\right)$ in permeability can also be ignored during calculation. Equation (6-15) makes it possible to assess the freckling potential using data of microsegregation, such as the relations of composition, temperature and fraction of solid in mushy region. These data of model alloys during solidification as experimentally determined in previous chapters are used to investigate their freckling tendencies due to composition effects. Since microsegregation increases with the decrease of temperature, $\mathrm{Ra}^{*}$ will vary with fraction solid along the depth of mushy zone, which will be illustrated in the next section.

Figure 6-3 shows the flowdown from the key control parameters to compositionrelated and processing-related freckling potentials. These two potentials must be combined to evaluate the total freckling tendency in superalloys. As stated previously, future work is needed to determine the composition effect on dendrite arm spacings.

\subsubsection{Freckling Tendencies in Model Alloys}

The density differences in the model alloys are plotted against temperature difference from liquidus in Figure 6-4. HV8852 exhibits greater density changes than HV8853, while HV8851 has inverted density gradient along the depth of mushy zone. If the dendrite arm spacings are neglected in the calculation of permeability, the permeability changes in the descending order of HV8853, HV8852, and HV8851, as shown in Figure 65. At this time, permeability is determined by fraction solid inside mushy zone. Since the density difference increases and the permeability decreases with the progress of solidification, it would be expected that the relative Rayleigh number reaches maximum in the mushy zone. Figure 6-6 and 6-7 show the $R a^{*}$ of the alloys versus temperature difference and fraction of solid respectively. The maximum $R a^{*}$ occurs at $10^{\circ} \mathrm{C}$ below 
liquidus for $\mathrm{HV} 8851,16^{\circ} \mathrm{C}$ for $\mathrm{HV} 8852$, and $26^{\circ} \mathrm{C}$ for $\mathrm{HV} 8853$. The fraction of solid at these temperatures is 0.3 to 0.4 for all of the three alloys. This is consistent with results of industrial castings [26] where freckles are likely to initiate at $15-20^{\circ} \mathrm{C}$ below the liquidus temperature at which the fraction solid is typically 0.4 to 0.6 . The $R a^{*}$ value of HV8853 is lower than HV8852, however, more freckle channels are observed in HV8853. It means that additional factors need to be considered to assessment their freckling potentials.

One of such important structural features is the secondary arm spacing $\left(d_{2}\right) . d_{2}$ of HV8852 is almost constant throughout the freezing process, while that of HV8853 continues to increase from the beginning to about $70 \%$ of total ingot solidification, as shown in Figure 3-16. The angle of solidification front $(\theta)$ also affects the freckling tendency. These values are reported in chapter 3 (HV8852 at 40 degrees and HV8853 at 30 degrees). $d_{2}$ and $\theta$ are included in the calculation of $R a$ and the maximum value versus distance from chill is shown in Figure 6-8. It is noted that the factor, $F(\theta, x, y)$, can not be calculated due to its implicit form. However, it also contributes to the freckling tendency.

As shown in Figure 6-8, the maximum value of $R a$ in HV8853 is smaller than that in HV8852 up to the mid-length of ingot where it begins to exceed that of HV8852. The magnitude of change in $R a$ in HV8853 is significantly greater than HV8852, which implicates that the severity of interdendritic liquid flow is enhanced due to the greater decrease of flow resistance. This is similar to the transients in remelt process when abrupt change in melting conditions leads to drastic changes of pool profile and dendrite arm spacings. Freckles are more likely to appear under these perturbed circumstances as compared with steady state solidification. It should be noted that Figure 6-8 shows the total Rayleigh number of model alloys under horizontal solidification condition, which combined both effects of composition and processing conditions.

No comparison of HV8851 was made due to the lack of dendrite arm spacing data. However, it would be expected that it has significantly larger dendrite arm spacing than $\mathrm{Nb}$-containing model alloys because of its mush slower cooling condition. The large dendrite arm spacing and the prolonged solidification time provided favorable conditions for freckle flow. Also the solidification front angle $(\theta)$ also increase the freckling potential as described by equation (6-8). 


\subsection{Freckling in Industrial Alloys}

The composition-related freckling index in equation (6-15) is applied to assess the freckling potential in industrial alloys 718, 706, and 625. Density difference and permeability (no dendrite arm spacings considered) vs. temperature drop from liquidus are shown in Figure 6-9 and 6-10. Liquid density change in alloy 718 is higher than those of alloy 625 and 706, while there is almost no density difference in alloy 706. The permeability of the alloys decreases in the order of 718, 706, and 625. These results are calculated from composition determined using systematic count method and fs(T) relation obtained using quantitative DTA.

The composition-related freckling index, $\mathrm{Ra}^{*}$, is shown against temperature drop and fraction of solid in Figures 6-11 and 6-12. It is clear that alloy 718 has the highest freckling potential among the three alloys. The maximum value occurs at $26^{\circ} \mathrm{C}$ below the liquidus with fs of 0.4 . Freckling potential in alloys 625 is lower than 718 but higher than 706. It occurs at $15^{\circ} \mathrm{C}$ below liquidus with fs of 0.36 (alloy 625). Freckling potential in alloy 706 is close to zero. The prediction agrees with industry experience that the limit of ingot size for the three alloys decreases in the order of 706, 625, and 718.

It is noted that for the large remelt ingot, processing conditions are also critical to prevent the formation of freckles. Both processing conditions and composition effect must be integrated to provide optimum solution for the production of freckle-free ingots.

\subsection{Discussion}

\subsubsection{Processing Conditions}

In previous contexts, we assume constant processing conditions. However, solidification rate $(R)$, solidification front angle $(\theta)$, and the pool geometry factor $F(\theta, x, y)$ in Rayleigh criterion are directly related to processing conditions (section 6.4). From the process point of view, the conditions can be categorized into steady state and non-steady state (transients). It is generally known that freckles are prone to form at high melt rates, while white spots are more likely to occur at low melt rates. Thus, there is a process window that is limited by the formation of these macrosegregation defects. Finding the optimum 
process window under steady state conditions is often the most critical step to produce a defect-free remelt ingot.

However, the solidification process will deviate away from steady state if there is any abrupt variation or transients. These transients result in significant perturbation of the flow fields in the mushy zone that lead to solute redistribution and, hence, macrosegregation. Many factors, such as poor electrode quality, transverse magnetic field, and melt rate excursion, contribute to transients. Here the melt excursion is used as an example to illustrate processing effects on the Rayleigh criterion. A melt rate excursion starts with gradual increase from the nominal steady state melt rate, followed by a rapid increase to a peak, a sudden decrease to a minimum, then gradually recover to the nominal melt rate. The peak variations were adopted from reference [2] and the steady state parameters taken from reference [8] for VAR alloy 718. The calculated thermal and dendritic parameters are given in Table 6-2. When the melt rate drops from maximum to minimum, the most noticeable changes are the decrease of pool depth at ingot edge and the increase of secondary dendrite arm spacing at ingot center. These changes result in a smaller $\theta$ angle (growth direction becomes more horizontal) and higher permeability, both of which will enhance the tendency for channel flow according to Rayleigh criterion.

\subsubsection{Ingot Size}

The larger the ingot, the smaller the processing window is to produce defect-free ingots. For a given alloy, there is a size limit beyond which it is difficult to maintain the ingot

quality. Better process control and modified alloy chemistry are two effective ways to increase the limit. As discussed in previous section, precise control of processing condition helps to reduce the fluctuation, thus, reduce the risk of freckling. The alloy chemistry can be modified such that the Ra in equation (6-8) is reduced. This will also decrease the total tendency for freckle formation provided the same processing conditions and ingot size. If the $\mathrm{Ra}$ is lower than the critical value for freckling, the ingot size can be increased without incurring freckle defects. For example, alloy A has a size limit of $\Phi_{1}$ (critical $R a=R_{1}$ ). If alloy $\mathrm{B}$ has a modified composition such that its $\mathrm{Ra}$ is smaller than $\mathrm{R}_{1}$ provided constant processing conditions, it can be produced with an ingot size larger than $\Phi_{1}$. 


\subsection{Summary}

The results and discussions are summarized as follows:

1. Rayleigh freckling criterion is developed for remelting process of Ni-based superalloys. It incorporates the key control parameters for freckle formation and processing conditions.

2. The freckling criterion can be broken down into composition-related and processrelated as those shown in section 6.4 .

3. Relative Rayleigh number, $\mathrm{Ra}^{*}\left(\mathrm{R}^{*}=\frac{\Delta \rho \Pi}{f_{L}}\right.$ ), is proposed to account for composition effects on freckle formation.

4. Liquid density difference depends on the nominal composition, elemental partition ratio, and the freezing range of alloys.

5. Significant thermal effect is found on liquid density difference. The large thermal effect is the intrinsic nature of Ni-based alloys.

6. Permeability of dendrite network depends on the characteristics of dendrites, i.e. dendrite arm spacings, fraction solid, tip curvature, and higher order dendrite arms.

7. $R a^{*}$ reaches maximum in HV8851, HV8852, and HV8853 at fraction solid of $0.3 \sim 0.4$ and temperature drop from liquidus of $16 \sim 26^{\circ} \mathrm{C}$.

8. $R a^{*}$ in industrial alloys decreases in the order of 718,625 , and 706 . The maximum $\mathrm{Ra}^{*}$ of alloys 718 and 625 occurs at fraction solid of $0.3 \sim 0.4$ and temperature drop from liquidus of $15^{\circ} \mathrm{C} \sim 26^{\circ} \mathrm{C}$.

9. The increase of freckling tendency with distance from chill is greater in HV8853 than in HV8852 if $d_{2}$ and $\theta$ are considered in calculation of Ra. This may contribute to higher tendency for freckling in HV8853 as observed in experiment.

10. Solidification transients, melt rate excursion in particular, affects the solidification front angle and the dendrite arm spacing. The abrupt change in dendrite morphology results in increase of permeability and higher potential of freckle formation.

11. The freckling criterion can be applied to increase the ingot size through precise process control and modification of alloy chemistry. 

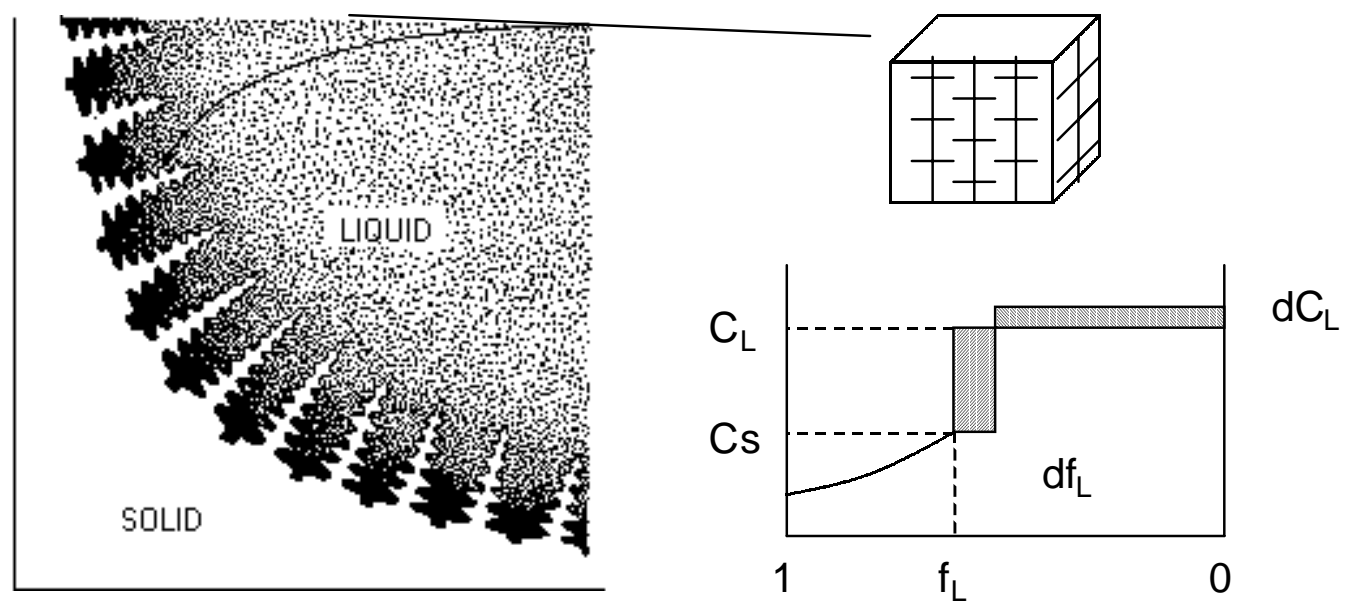

Figure 6-1: Schematic illustration of volume element during remelt solidification and mass balance in the volume element inside mushy zone.

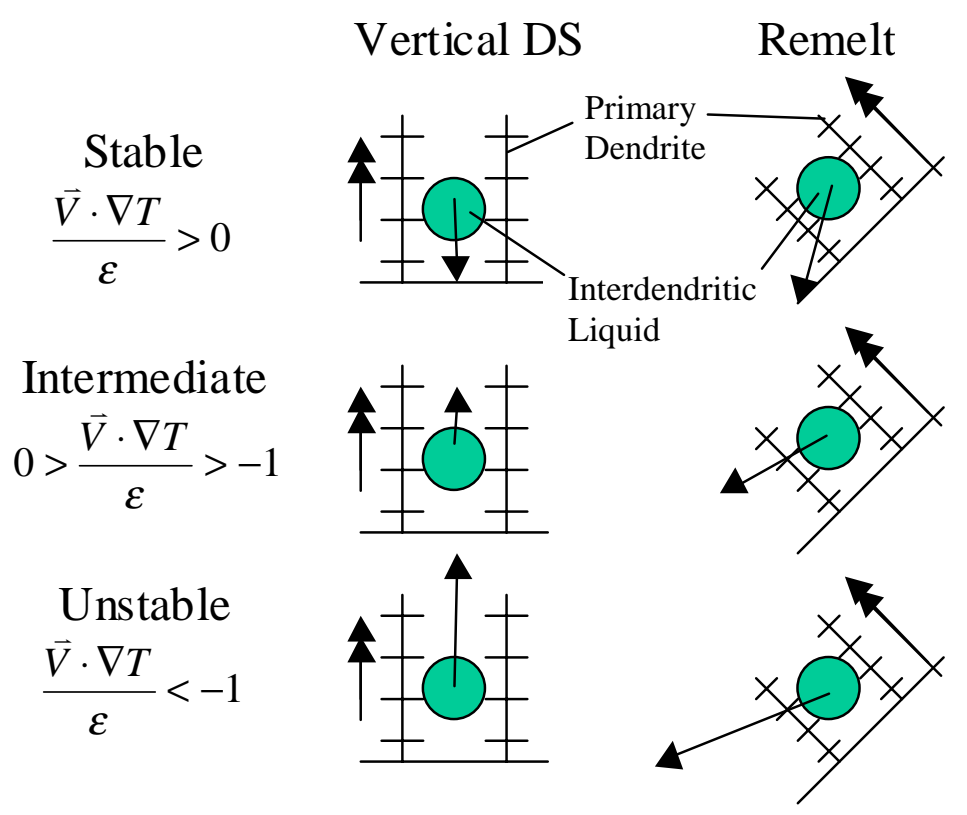

Figure 6-2: Schematic illustration of change in flow behavior in the mushy zone during solidification under vertical DS (with density inversion) and remelt (without density inversion) conditions. (double arrow is the growth direction of primary dendrites, single arrow is the flow direction of the interdendritic liquid.) 


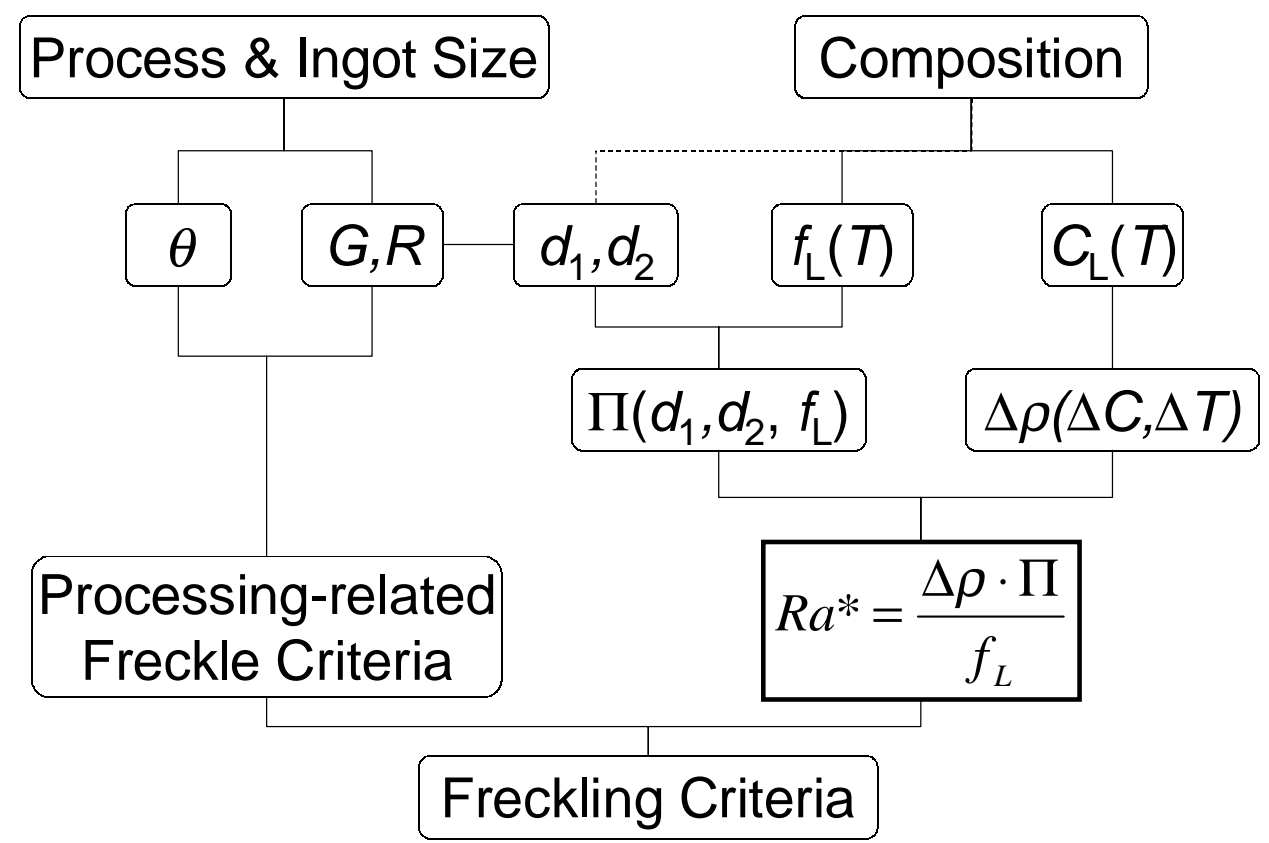

Figure 6-3: Flowdown of controlling factors of freckling in remelt processing of superalloys.

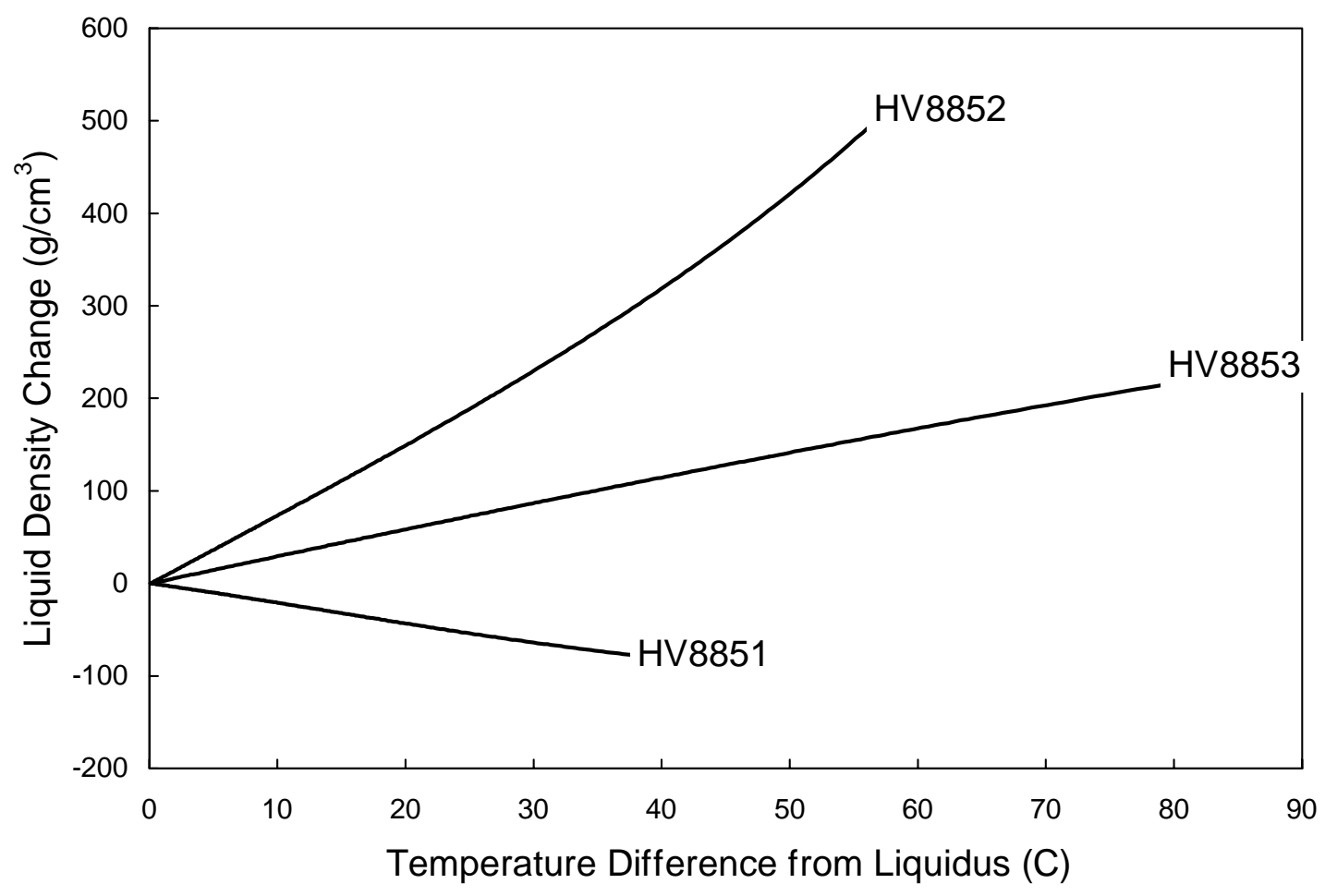

Figure 6-4: Liquid density change versus temperature difference of model alloys. 


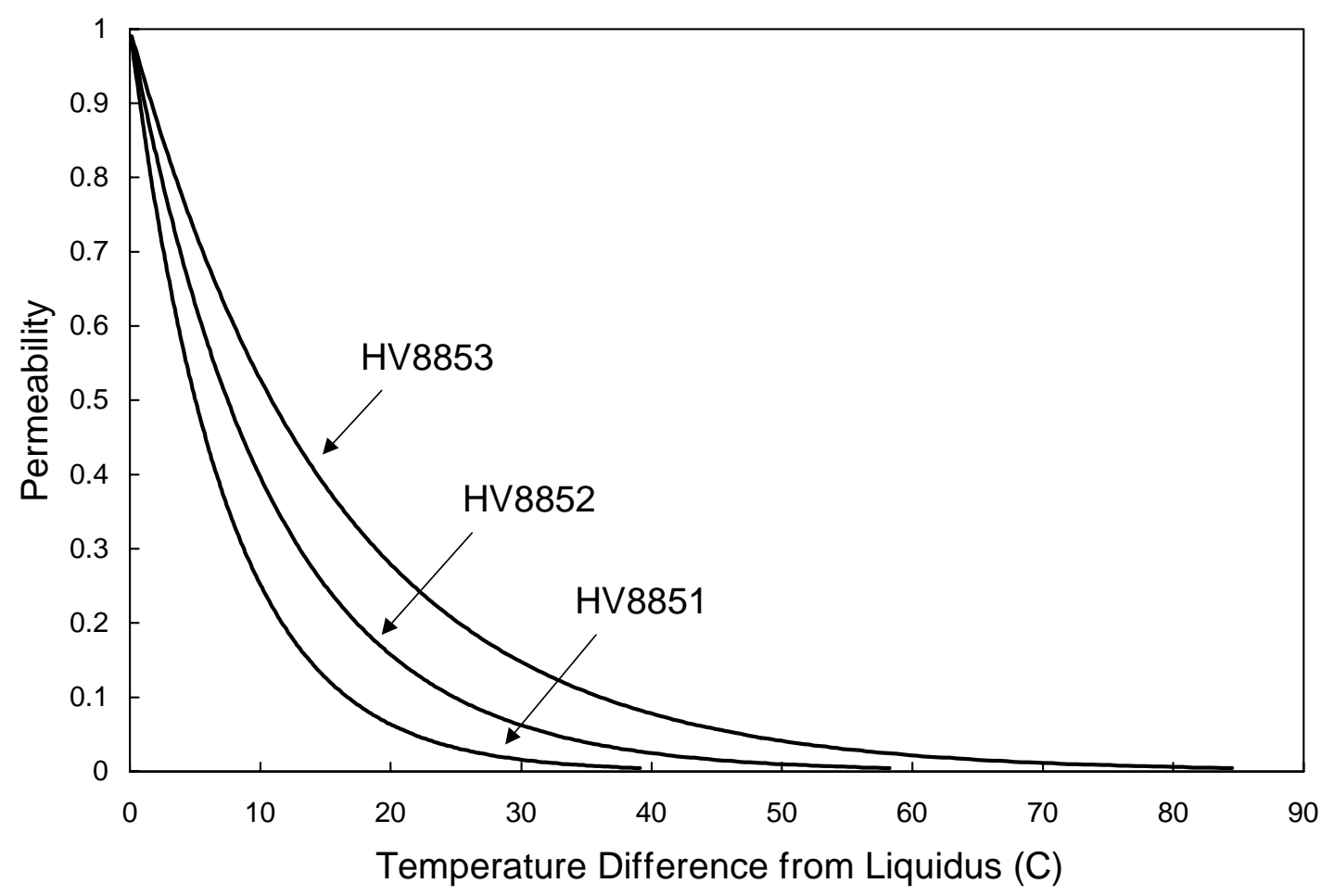

Figure 6-5: Permeability versus temperature difference of model alloys (no $d_{2}$ considered).

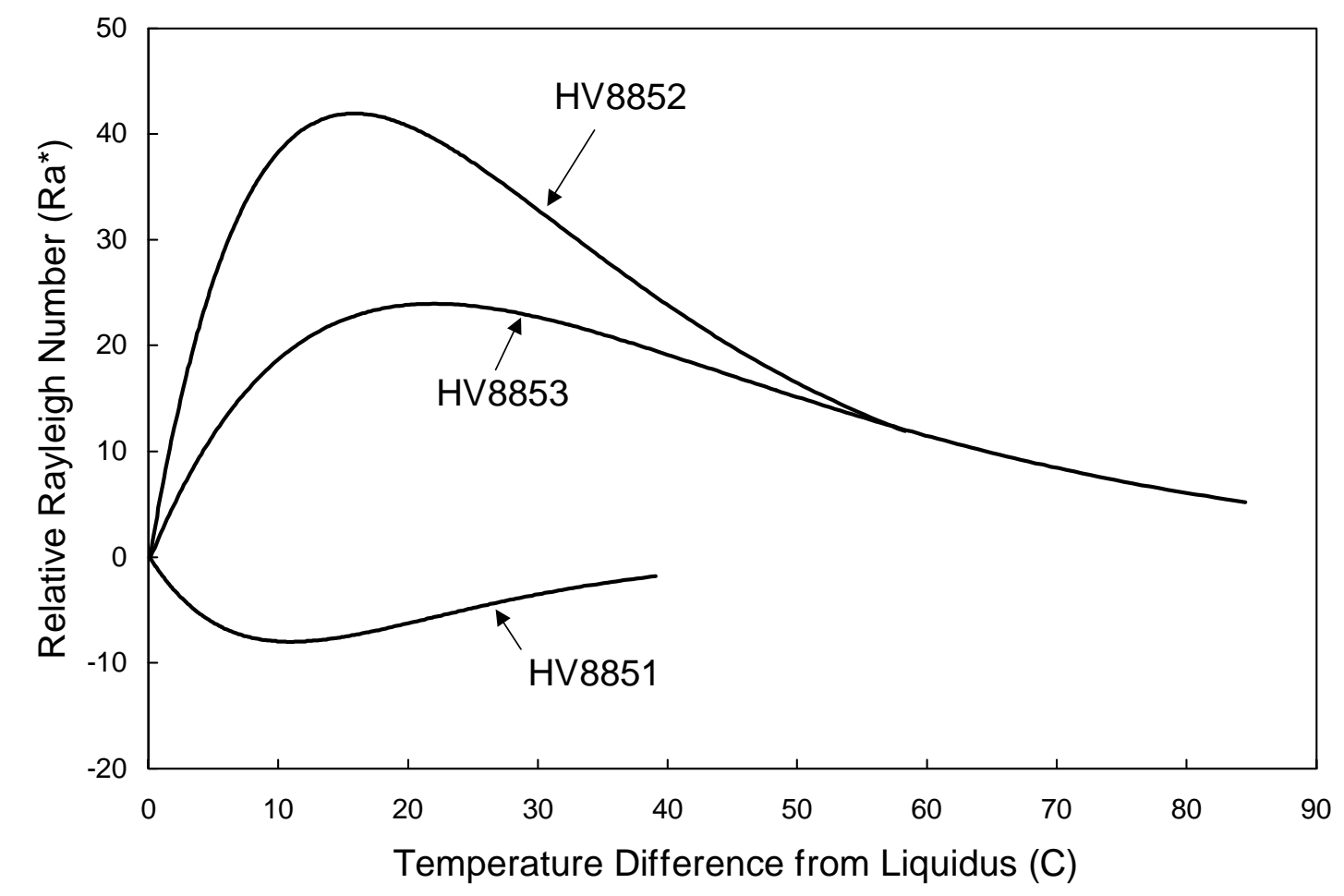

Figure 6-6: Relative Ra* versus temperature difference of model alloys (no $d_{2}$ considered). 


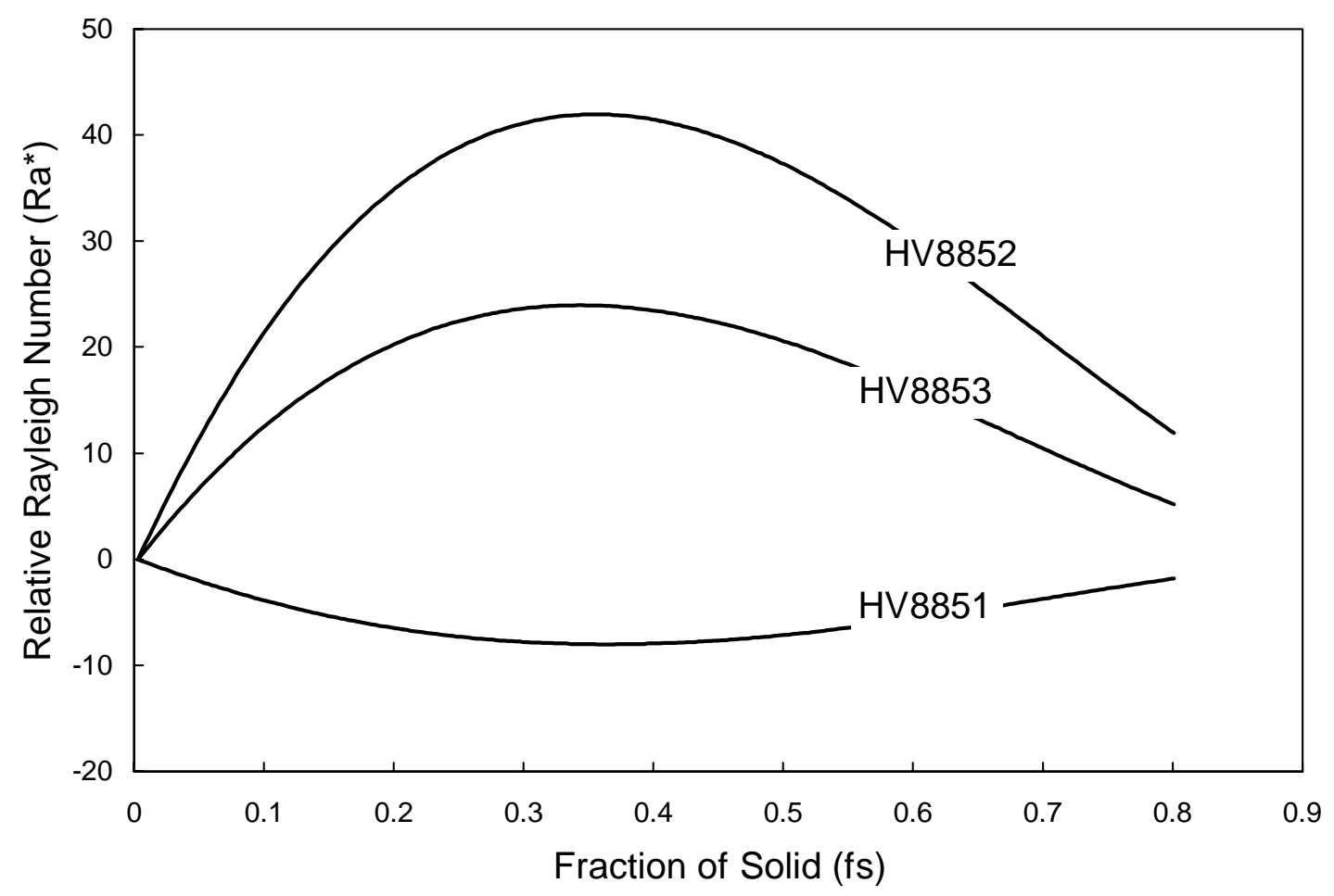

Figure 6-7: $\mathrm{Ra}^{*}$ versus fraction of solid of model alloys (no $d_{2}$ considered).

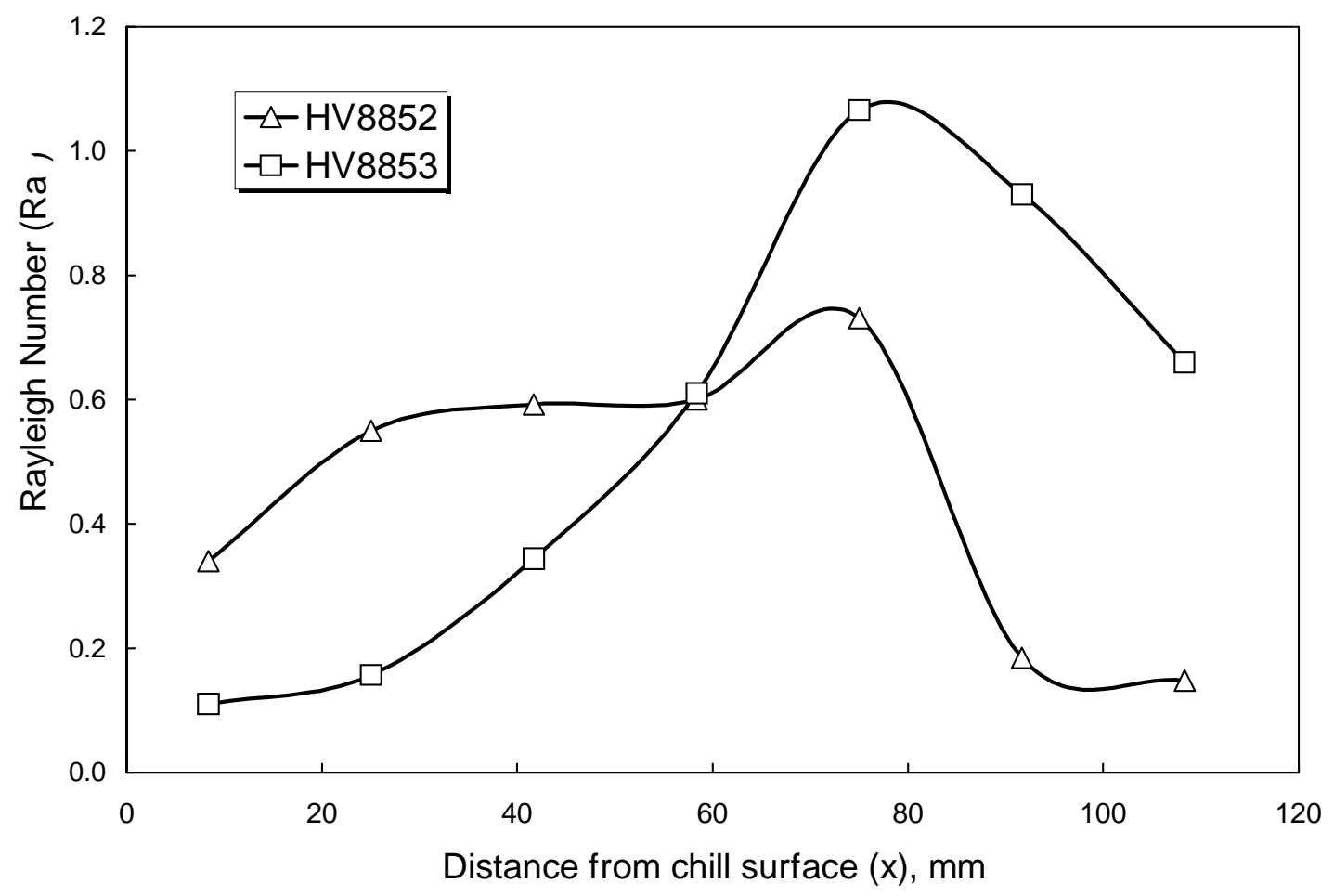

Figure 6-8: Maximum Ra versus distance from chill of horizontally solidified model alloys HV8852 and HV8853 ( $d_{2}$ and $\theta$ are considered in calculation). 
Table 6-2: Melt Rate Excursion of VAR 718

\begin{tabular}{|c|c|c|c|l|}
\hline \multicolumn{2}{|c|}{ Melt Parameters } & Center & Edge & Units \\
\hline \multirow{4}{*}{ Max } & $\mathrm{R}$ & 3.58 & 3.58 & $\mathrm{~mm} / \mathrm{min}$ \\
\cline { 2 - 5 } & LST & 41.85 & 28.23 & $\mathrm{~min}$ \\
\cline { 2 - 5 } & pool depth & 149.93 & 101.12 & $\mathrm{~mm}$ \\
\cline { 2 - 5 } & $\mathrm{G}$ & 0.43 & 0.63 & ${ }^{\circ} \mathrm{C} / \mathrm{mm}$ \\
\cline { 2 - 5 } & cool rate & 1.53 & 2.27 & ${ }^{\circ} \mathrm{C} / \mathrm{min}$ \\
\cline { 2 - 5 } & SDAS & 117.01 & 102.42 & $\mathrm{micron}$ \\
\hline \multirow{4}{*}{ Min } & $\mathrm{R}$ & 2.48 & 2.48 & $\mathrm{~mm} / \mathrm{min}$ \\
\cline { 2 - 5 } & LST & 55.15 & 28.23 & $\mathrm{~min}$ \\
\cline { 2 - 5 } & pool depth & 136.97 & 70.10 & $\mathrm{~mm}$ \\
\cline { 2 - 5 } & $\mathrm{G}$ & 0.47 & 0.91 & ${ }^{\circ} \mathrm{C} / \mathrm{mm}$ \\
\cline { 2 - 5 } & cool rate & 1.16 & 2.27 & ${ }^{\circ} \mathrm{C} / \mathrm{min}$ \\
\cline { 2 - 5 } & SDAS & 128.45 & 102.42 & $\mathrm{micron}$ \\
\hline
\end{tabular}

Diameter $=508 \mathrm{~mm}, \Delta \mathrm{T}=64^{\circ} \mathrm{C}$, Nominal melt rate $=276 \mathrm{~kg} / \mathrm{hr}$, Liquid density $=7486 \mathrm{~kg} / \mathrm{m}^{3}$, Ingot growth rate $(\mathrm{R})=3.03 \mathrm{~mm} / \mathrm{min}$, Variation of melt rate $=50 \mathrm{~kg} / \mathrm{hr}$. LST $=$ local solidification time.

Center: SDAS $=123.24$ micron, pool depth $=102.42 \mathrm{~mm}$ Edge: SDAS $=148$ micron, pool depth $=85.61 \mathrm{~mm}$

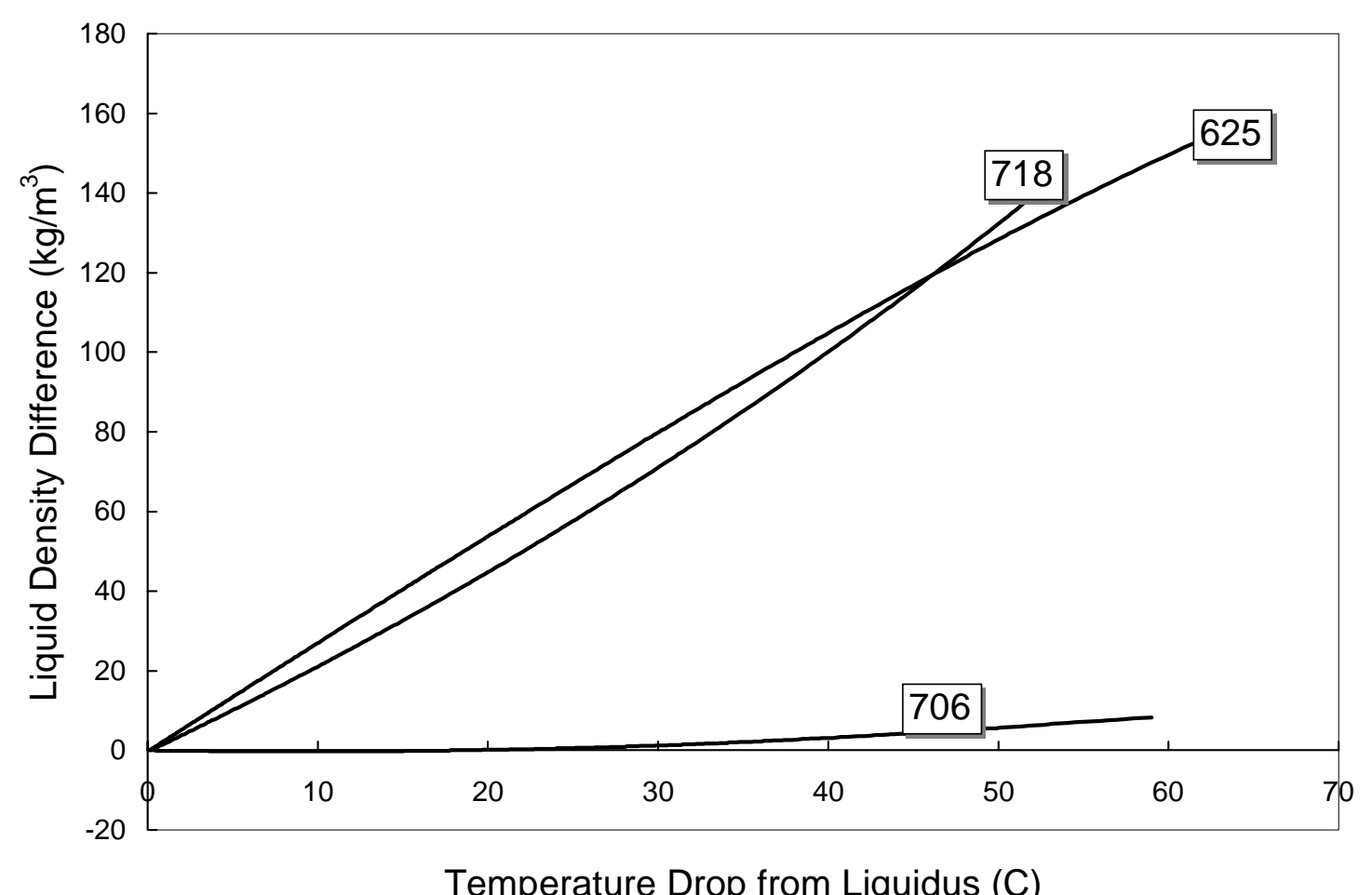

Figure 6-9: Liquid density change versus temperature difference of industrial alloys. 


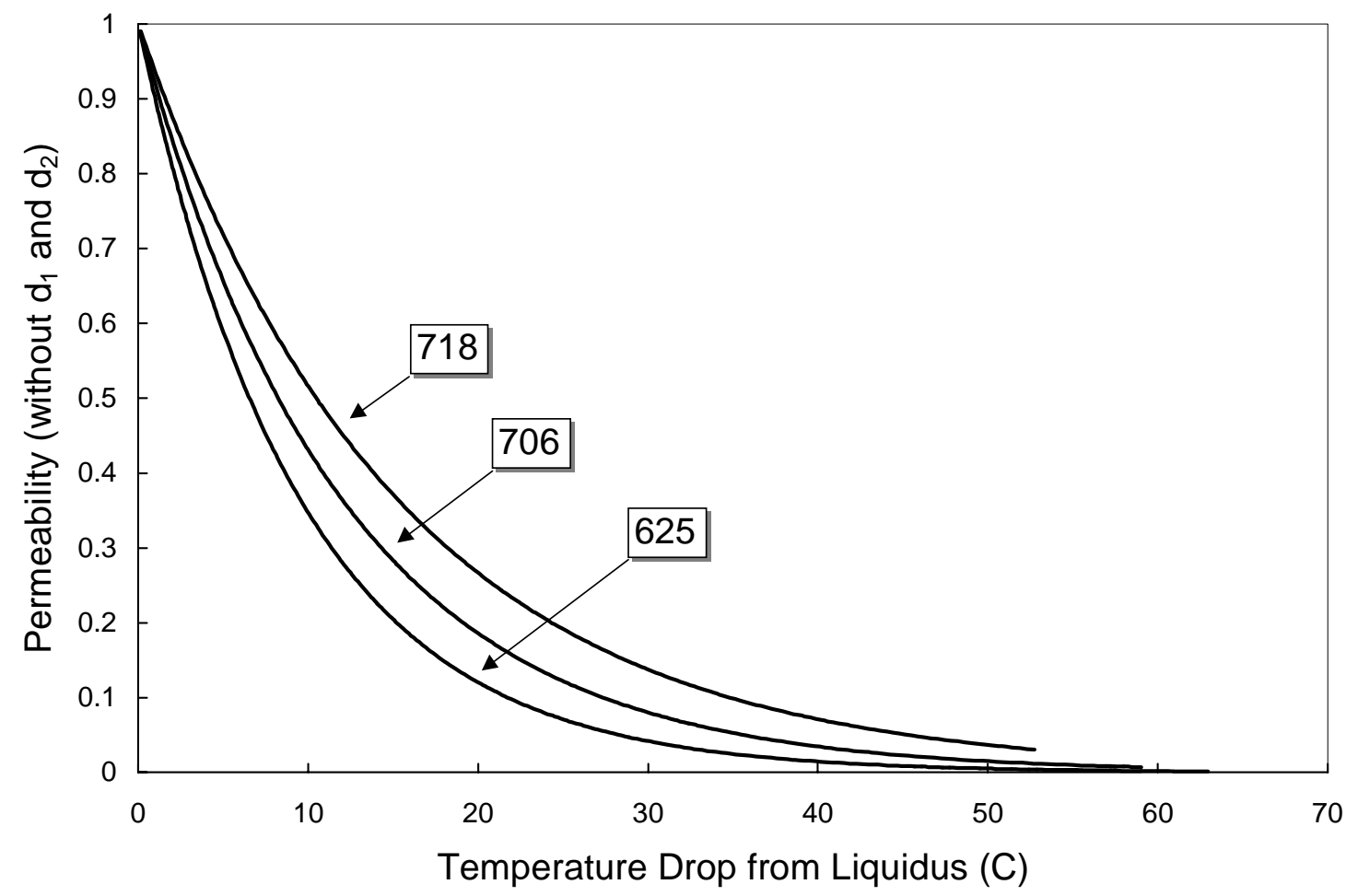

Figure 6-10: Permeability vs. temperature difference of industrial alloys (no $d_{2}$ considered).

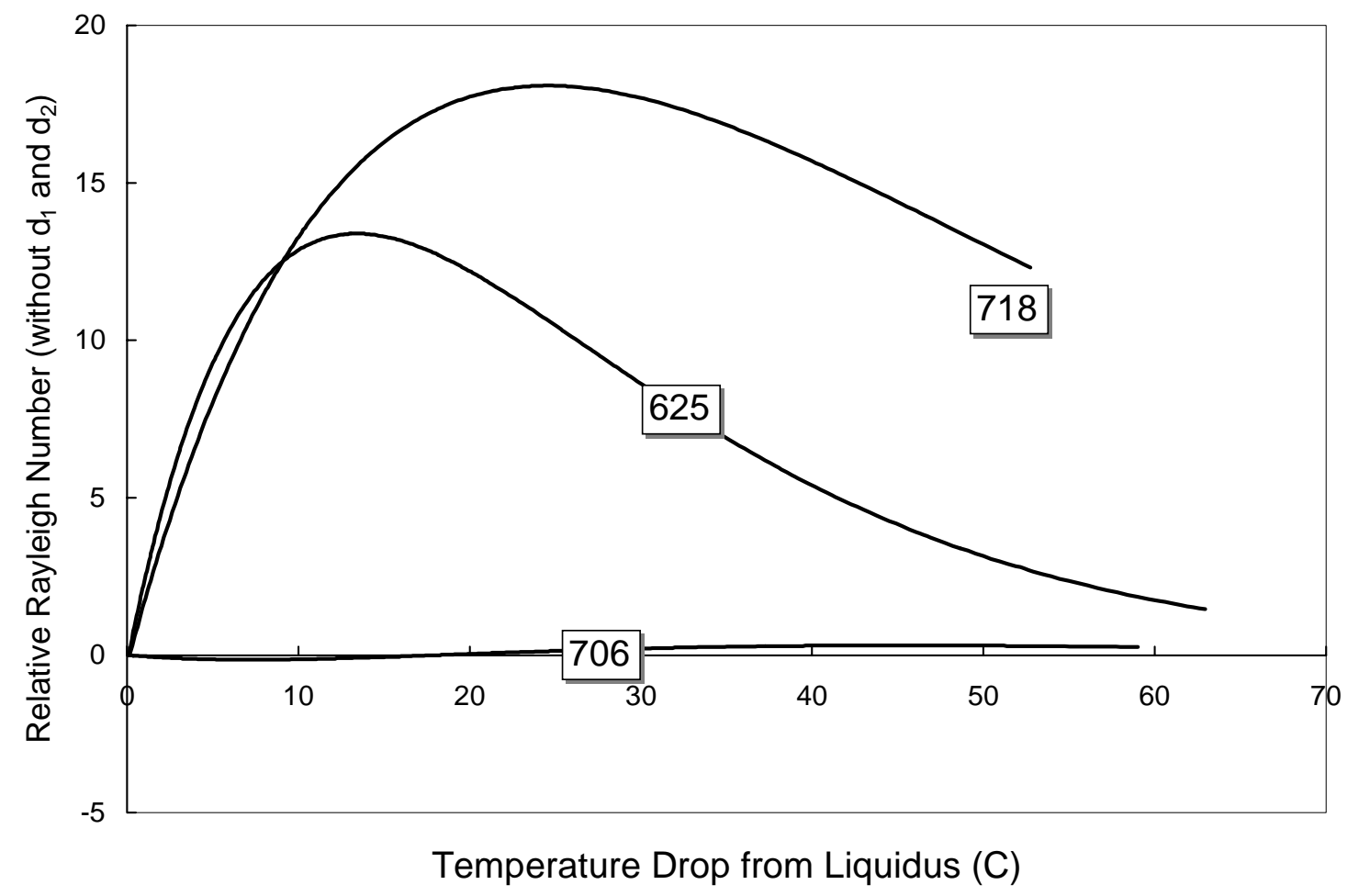

Figure 6-11: Relative Ra* vs. temperature difference of industrial alloys (no $d_{2}$ considered). 


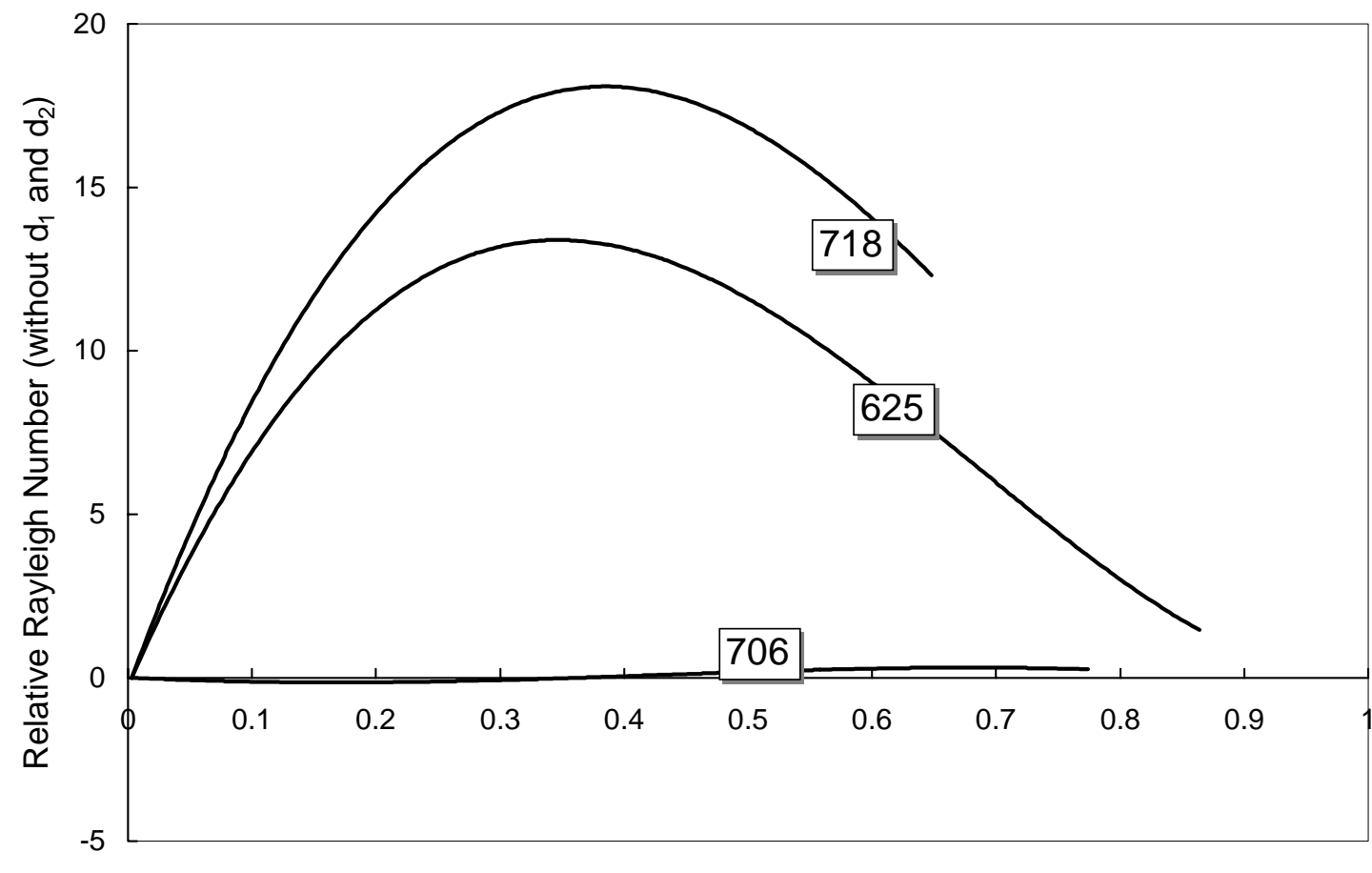

Fraction Solid (fs)

Figure 6-12: Ra* versus fraction of solid of model alloys (no $d_{2}$ considered). 


\section{Chapter 7}

\section{Conclusions}

\subsection{Conclusions}

Following conclusions are drawn from the present investigation:

1. Freckles in Ni-based superalloys are caused by density-driven interdendritic fluid flow. Downward flow of interdendritic liquid that is denser than bulk liquid is responsible for freckling in Nb-containing alloys, while liquid density inversion accounts for freckling in Ti-rich alloys.

2. Freckles initiate at irregularities in the dendritic region and are likely to originate from the primary dendrite space. Freckle channel tends to align itself in the direction with high gravity potential and permeability. The iso-fraction solids provide the maximum space for freckle flow.

3. Key control parameters, namely the liquid density gradient inside mushy region, the freezing range $\left(\Delta T=T_{L}-T_{S}\right)$, the fraction of solid $\left(f_{S}\right)$, primary and secondary dendrite arm spacings $\left(d_{1} \& d_{2}\right)$, and solidification front angle $(\theta)$, for freckling in remelt Ni-based superalloys are identified.

4. Composition effects on fraction of liquid curve, $f_{L}(T)$, is determined as follows:

$$
\alpha=-0.0397+0.002836 \cdot[\mathrm{Nb}]+0.002131 \cdot[\mathrm{Ti}]
$$

Where $f_{L}=e^{-\alpha \cdot\left(T-T_{L}\right)}$ and terms in brackets are nominal alloy compositions in wt pct. The equation applies to following composition ranges: $[\mathrm{Cr}]=20,[\mathrm{Fe}]=18$ or $36,[\mathrm{Al}]=0$ or $1,3<[\mathrm{Nb}]<7.8$ and $0.3<[\mathrm{Ti}]<5.6$, all in wt pct. $\mathrm{Nb}$ is seen to have more pronounced effect than Ti in increasing the fraction of liquid.

5. Rayleigh criterion incorporating the key control parameters is developed to account for freckling in remelt process:

$$
R a=F(\theta, x, y) \cdot \frac{\Delta \rho g \Pi}{\eta f_{L}} \cdot \frac{1}{R \sin \theta}>R c
$$

Composition effects can be illustrated by using relative Rayleigh number: 


$$
R a^{*}=\frac{\Delta \rho \Pi}{f_{L}}>R c^{*}
$$

6. Solidification diagram of Ni-Cr-Fe-Nb alloy system is developed via CALPHAD method. Increase of $[\mathrm{Cr}+\mathrm{Fe}]$ and/or $\mathrm{Nb}$ contents favor the formation of Laves phase in the alloy. The predicted ternary eutectic reaction, $L-(\gamma+\delta+$ Laves $)$, is validated by observations in experimental model alloys.

7. Experimental techniques, namely systematic point count EDS and quantitative DTA analysis, are developed to determine the composition, fraction solid, and temperature relations during solidification of multicomponent superalloys.

\subsection{Contributions}

The most significant contributions of this work are

1) Identification of the key control parameters for freckle formation under horizontal solidification conditions. It is based upon the successful physical simulation of freckling in model alloys and the careful examination of freckles in both model alloys and industrial alloy. The key control parameters can be readily applied to remelting conditions since the physical simulation correctly represents the horizontal component in remelt ingot where the freckling potential is the highest.

2) Discovery of the alloying effect on the liquid density change and fraction of solid during solidification of superalloys. The key composition variables studied are $\mathrm{Nb}$ and $\mathrm{Ti}$, which are major strengthening elements in freckle-prone industrial alloys.

3) Development of simple and accurate techniques for the determination of the solidification characteristics in multicomponent superalloys. The methods can significantly improve the sensitivity of the freckling criterion and reduce the time needed for measurement.

4) Development of the solidification diagram of $\mathrm{Ni}-\mathrm{Cr}-\mathrm{Fe}-\mathrm{Nb}$ alloy system using CALPHAD approach. The diagram is capable to predict the solidification sequence and the alloying effects on secondary phases. More confidence is gained in applying CALPHAD method to solidification simulations. 


\section{Appendix A:}

\section{Solute Redistribution Model in Solidification}

\section{Equation (1-10)}

For usual casting and ingot solidification, microsegregation can be accurately predicted by the solute redistribution equation provided there is no diffusion in the solid and complete liquid mixing. For the volume element shown in Figure 6-1, assuming no mass exchange with the surrounding environment, the mass balance in the volume element can be written as:

$$
(1-k) C_{L} d f_{L}=-f_{L} d C_{L}
$$

Where $C_{L}$ is the liquid composition within the volume element, $f_{L}$ is the fraction of

liquid in the volume element. $k$ is the equilibrium partition ratio, $k=C_{S}^{*} / C_{L}$, where $C_{S}^{*}$ is the interface solid composition. Integrate both sides of (A-1):

$$
C_{S}^{*}=k C_{0}\left(1-f_{S}\right)^{k-1}
$$

The equation (A-2), for the case of no solid diffusion, describes solid composition as a function of fraction solid during solidification. It therefore can be used to describe the severity of microsegregation.

\section{Equation (6-1)}

Here mass flow in or out of the volume element during the solidification is considered. Solute enters or leaves the element by diffusion is neglected.

Conservation of solute mass in the volume element during solidification requires:

$$
\frac{\partial}{\partial t}(\bar{\rho} \bar{C})=-\nabla \cdot \rho_{L} f_{L} C_{L} \vec{V}
$$

Where is the local average density, is the local average composition of liquid and solid, is the liquid density, is the local velocity of interdendritic liquid relative to solid.

Conservation of total mass in the volume element gives: 


$$
\frac{\partial \bar{\rho}}{\partial t}=-\nabla \cdot \rho_{L} f_{L} \vec{V}
$$

Expanding the right side of equation (A-4):

$$
\frac{\partial}{\partial t}(\bar{\rho} \bar{C})=-C_{L} \nabla \cdot \rho_{L} f_{L} \vec{V}-\rho_{L} f_{L} \vec{V} \cdot \nabla C_{L}
$$

And substituting equation (A-4):

$$
\frac{\partial}{\partial t}(\bar{\rho} \bar{C})=C_{L} \frac{\partial \bar{\rho}}{\partial t}-\rho_{L} f_{L} \vec{V} \cdot \nabla C_{L}
$$

The change in solute mass of the volume element per unit time is the sum of the changes of the liquid and solid phases:

$$
\frac{\partial}{\partial t}(\bar{\rho} \bar{C})=\frac{\partial}{\partial t}\left(\rho_{S} f_{S} \bar{C}_{S}+\rho_{L} f_{L} C_{L}\right)
$$

Where $\bar{C}_{S}$ is the local average composition of the solid, $\rho_{S}$ is the solid density. Now assume the equilibrium partition ratio, $k$, applies at the liquid-solid interface, no diffusion in the solid, and solid density is constant during solidification.

Then:

$$
\frac{\partial}{\partial t}\left(\bar{C}_{S} \rho_{S} f_{S}\right)=k C_{L} \rho_{S} \frac{\partial f_{S}}{\partial t}
$$

and if no pores form:

$$
\begin{aligned}
& f_{S}+f_{L}=1 \\
& d f_{S}=-d f_{L}
\end{aligned}
$$

Substituting equations (A-8) and (A-9) in (A-7):

$$
\frac{\partial}{\partial t}(\bar{\rho} \bar{C})=-k C_{L} \rho_{S} \frac{\partial f_{L}}{\partial t}+\rho_{L} f_{L} \frac{\partial C_{L}}{\partial t}+C_{L} \frac{\partial\left(\rho_{L} f_{L}\right)}{\partial t}
$$

An expression similar to equation (A-7) is now written for the total mass of the element:

$$
\frac{\partial}{\partial t}(\bar{\rho})=\frac{\partial}{\partial t}\left(\rho_{S} f_{S}+\rho_{L} f_{L}\right)
$$

Substituting (A-9), (A-10) and (A-11) in (A-6) and rearranging:

$$
-k C_{L} \rho_{S} \frac{\partial f_{L}}{\partial t}+\rho_{L} f_{L} \frac{\partial C_{L}}{\partial t}=-C_{L} \rho_{S} \frac{\partial\left(\rho_{L}\right)}{\partial t}-\rho_{L} f_{L} \vec{V} \cdot \nabla C_{L}
$$

or 


$$
\frac{\partial C_{L}}{\partial t}=-\left(\frac{1-k}{1-\beta}\right) \frac{C_{L}}{f_{L}} \frac{\partial f_{L}}{\partial t}-\vec{V} \cdot \nabla C_{L}
$$

where $\beta$ is the solidification shrinkage:

$$
\beta=\frac{\rho_{S}-\rho_{L}}{\rho_{S}}
$$

From chain rule,

$$
\nabla C_{L}=\frac{\nabla T}{\varepsilon} \frac{\partial C_{L}}{\partial t}
$$

$\varepsilon$ is the cooling rate. Combining equations (A-13) and (A-15):

$$
\frac{\partial f_{L}}{\partial C_{L}}=-\left(\frac{1-\beta}{1-k}\right)\left(1+\frac{\vec{V} \cdot \nabla T}{\varepsilon}\right) \frac{f_{L}}{C_{L}}
$$

The above equation is used to describe the local solute redistribution considering the interdendritic liquid flow by solidification shrinkage. The flow instability criterion from equation (A-16) can also be applied to the liquid flow caused by density inversion as described in chapter 6 . 


\section{Appendix B:}

\section{Square-Mesh Systematic Point Count Metallographic Technique Combined with Energy Dispersive Spectrometry to Investigate Microsegregation}

Microsegregation in Ni-based superalloys provides the fundamental knowledge to understand the macrosegregation behavior, e.g. freckling, of the alloys. A simple, statistically significant experimental technique, originally developed by Gungor [46], is improved in the current study to measure the solute redistribution profiles in solidified $\mathrm{Ni}$ based alloys using Electron Dispersive Spectrometry (EDS) chemistry analysis.

A two dimensional coarse square mesh with an average step size (the distance between two consecutive measurements) of 60 100 microns was used in the measurement. At least 4 fields were chosen for each sample. A total of about 150 measurements per sample were obtained. The manner of sampling and sample size (number of measurements) were chosen in order to obtain statistically significant results according to Gungor.

The data were processed in the following manner and result of a DTA sample of alloy 718 is used to demonstrate the procedure:

1. The as-measured compositions were plotted versus their data number (Figure B-1 (a)).

2. The scattered composition data were put in ascending (or descending) order and the sorted compositions versus ordered data number were plotted.

3. Ordered data number axis (x-axis) was converted to volume fraction by dividing each data number by total data number. The composition versus volume fraction solid was then plotted.

4. Volume fraction of solid axis was converted into weight fraction solid using the following equation,

$$
f_{S i}=\sum_{j=1}^{i} \rho_{j} \cdot \Delta V_{S j} / \rho
$$

where $f_{S i}$ and $V_{S i}$ are the weight and volume fraction of datum $i . \rho_{j}$ is the density $\left(\mathrm{g} / \mathrm{cm}^{3}\right)$ of datum $j, \rho$ is the average density of all data. Since for Ni-based alloys $\rho_{i} \approx \rho$ (Knorovsky 1989 and current study), $f_{S i}=V_{S i}$ can be assumed (Figure B-1 (b)). 
5. The solid composition versus weight fraction solid data was fitted using the following equation, $y=a+b x+c x^{2}, \quad y=\ln \left(C_{S} / C_{0}\right), \quad x=\ln \left(f_{L}\right), \quad f_{L}=1-f_{S}$.

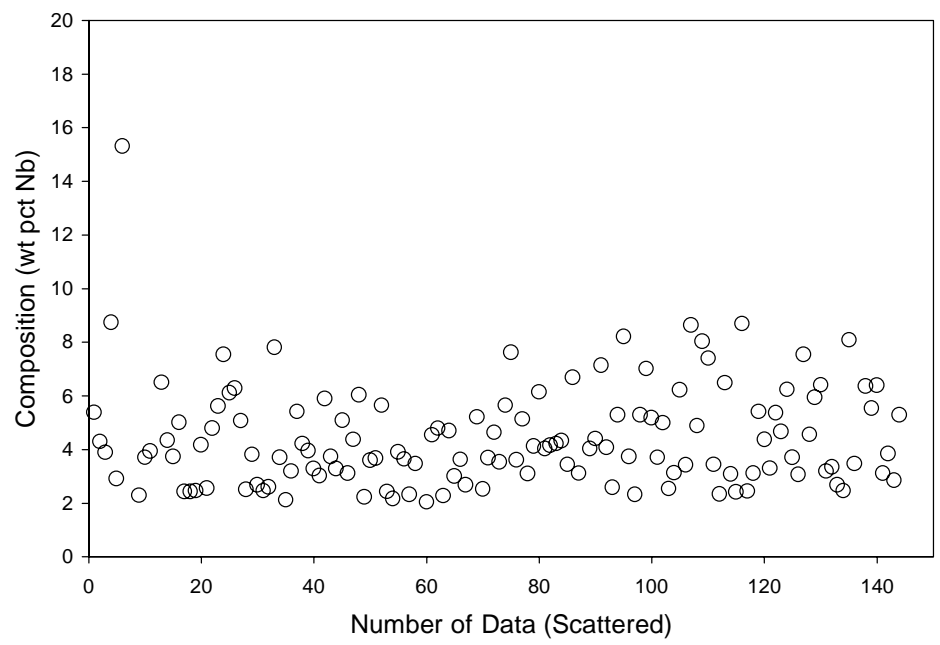

(a)

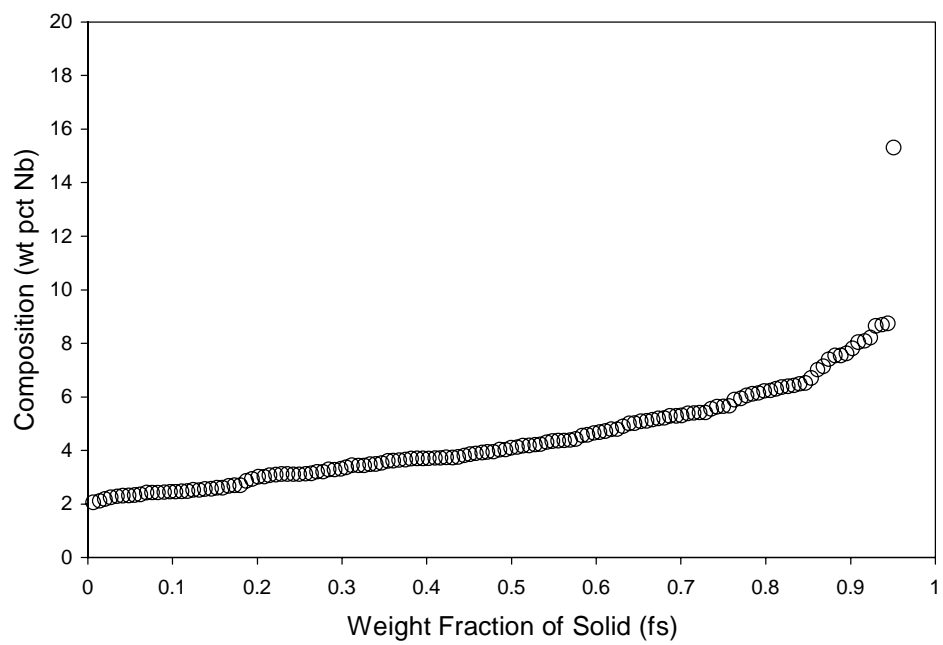

(b)

Figure B-1: (a) Nb composition versus scattered data and (b) Nb composition versus fraction solid profile obtained in alloy 718 DTA sample. 


\section{Reference}

1 R. C. Schwant, et al.

Large 718 Forgings for Land Based Turbines

Superalloys 718, 625, 706 and Various Derivatives, ed. E. A. Loria, TMS, 1997, 141-152

2 A.D. Helms, C. B. Adasczik and L. A. Jackman

Extending the Size Limit of Cast/Wrought Superalloy Ingots

Superalloys 1996, ed. R. D. Kissinger et al, TMS, 1996, 427-433

3 K. O. Yu and J. A. Domingue

Control of Solidification Structure in VAR and ESR Processed Alloy 718 Ingots

Superalloy 718-Metallurgy and Applications, ed. E. A. Loria, TMS, 1989, 33-48

4 L. A. Jackman, G. E. Maurer and S. Widge

New Knowledge about “ White Spots" in Superalloys

Advanced Materials \& Processes, May 1993, 18-25

5 A.F. Giamei and B. H. Kear

On the Nature of Freckles in Nickel Base Superalloys

Metall. Trans., 1970, v. 1, 2185-2192

6 S. M. Copley, A. F, Giamei, S. M. Johnson and M. F. Hornbecker

The Origin of Freckles in Unidirectionally Solidified Castings

Metall. Trans., 1970, v. 1, 2193-2203

7 T.M. Pollock and W.H. Murphy

The Breakdown of Single Crystal Solidification in High Refractory Ni-base Alloys

Metall. Trans. A, 1996, v. 27A, 1081-1094

8 K. O. Yu, J. A, Domingue, G. E. Maurer and H. D. Flanders

Marco segregation in ESR and VAR Processes

JOM, Jan. 1986, 46-50

9 S. Thamboo

Melt Related Defects in Alloy 706 and Their Effects on Mechanical Properties Superalloy 718, 625, 706 and Various Derivatives, ed. E.A. Loria, TMS 1994, 137-152

10 M. C. Flemings and G. E. Nereo

Macrosegregation: Part I

Trans. AIME, 1967, v.239, 1449-1461

11 M. C, Flemings, R. Mehrabian and G. E. Nereo

Macrosegregation: Part II

Trans. AIME, 1968, v.242, 41-49

12 M. C, Flemings and G. E. Nereo

Macrosegregation: Part III

Trans. AIME, 1968, v.242, 50-55

13 R. Mehrabian, M. Keans and M. C, Flemings

Interdendritic Fluid Flow and Macrosegregation; Influence of Gravity

Metall. Trans., 1970, v. 1, 1209-1220

14 R. J. McDonald and J. D. Hunt

Convective Fluid Motion within the Interdendritic Liquid of a Casting

Metall. Trans., 1970, v. 1, 1787-1788

15 R. J. McDonald and J. D. Hunt

Fluid Motion through the Partially Solid Regions of a Casting and Its Importance in

Understanding A Type Segregation

Trans. AIME, 1969, v.245, 1993-1997

16 N. Stewart and F. Weinberg

Pipe Formation in $\mathrm{Pb}-\mathrm{Sn}$ Alloys

Metall. Trans., 1972, v. 3, 3181-3184

17 J. Verhoeven

Macrosegregation during Growth of a Dendrite Array into a Stirred Melt at Near Steady-State Conditions Metall. Trans., 1971, v. 2, 16783-2680

18 N. Stewart and F. Weinberg 
Interdendritic Fluid Flow in a Lead-Tin Alloy

Metall. Trans. B, 1976, v. 7B, 417-423

19 M. J. Stewart, L. C. MacAulay and F. Weinberg

Macrosegregation in Castings Rotated and Oscillated during Solidification

Metall. Trans., 1971, v. 2, 169-173

20 F. Kaempffer and F. Weinberg

Macrosegregation in a Copper Alloy Directionally Cast with Exudation of Liquid

Metall. Trans., 1971, v. 2, 2477-2483

21 G. Hansen, A. Hellawell, S. Z. Lu and R. S. Stuebe

Some Consequences of Thermosolutal Convection: The Grain Structure of Castings

Metall. Trans. A, 1996, v. 27A, 569-581

22 J. R. Sarazin and A. Hellawell

Channel Formation in $\mathrm{Pb}-\mathrm{Sn}, \mathrm{Pb}-\mathrm{Sb}$ and $\mathrm{Pb}-\mathrm{Sn}-\mathrm{Sb}$ Alloy Ingots and Comparison with $\mathrm{NH}_{4} \mathrm{Cl}-\mathrm{H} 2 \mathrm{O}$

Metall. Trans. A, 1988, v. 19A, 1861-1871

23 H. Song, et al.

Convection during Thermal Unstable Solidification of $\mathrm{Pb}-\mathrm{Sn}$ in a Magnetic Field

Metall. Trans. A, 1996, v. 27A, 1095-1110

24 A.K. Sample, A. Hellawell, and R.S. Steube

Channel Convection in Partly Solidified Systems

Phi. Trans. R. Soc. Lon. A, 1993, v.345, 507-544

25 J.A. Van Den Avyle, J.A. Brooks, and A.C. Powell

Reducing Defects in Remelting Processes for High Performance Alloys

JOM, Mar. 1998, 22-25

26 P. Auburtin and A. Mitchell

Elements of Determining of a Freckling Criterion

Liquid Metal Processing Symposium, 1997, 18-34

27 D.K. Melgaard, R.L. Williamson, and J.J. Beaman

Controlling Remelting Processes for Superalloys and Aerospace Ti Alloys

JOM, Mar. 1998, 13-17

28 F. J. Zanner, L. A. Bertram, B. Harrison and H. D. Flanders

Relationship between Furnace Voltage Signatures and the Operational Parameters Arc

Power, Arc Current, CO Pressure, and Electrode Gap during Vacuum Arc Melting INCONEL 718

Metall. Trans. B, 1986, v. 17B, 357-365

29 T. M. Pollock, W. H. Murphy, E. H. Goldman, D. L. Uram and J. S. Su

Grain Defect Formation during Directional Solidification of Nickel Base Single Crystals

Superalloys 1992, ed. S. D. Antolovich et al, TMS, 1992, 125-134

30 N. Mori and K. Ogi

Study on Formation of Channel-Type Segregation

Metall. Trans. A, 1991, v. 22A, 1663-1672

31 W. Kurz and D.J. Fisher

Acta metall., 1981, vol.29, 11-20

32 M. C. Flemings

Solidification Processing

McGraw-Hill, Inc., 1974

33 J.S. Kirkaldy and W. Y. Youdelis

Contribution to the Theory of Inverse Segregation

Trans. AIME, 1961, v,221, 833-840

34 G. C. Gould

Freckle Segregation in Vacuum Consumable-Electrode Ingots

Trans. AIME, 1965, v.233, 1345-1351

35 R. P. DeVries and G. R. Mumau

Importance of Relationship between Dendrite Formation and Solidification in Highly Alloyed Materials JOM, Nov. 1968, 33-36

36 P.K. Sung and D.R. Poirier

Liquid-Solid Partition Ratios in Ni-base Alloys

Metall. Trans. A, 1999, v. 30A, 2173-2181 
37 K-M Chang, H-J Lai and J-Y Hwang

Existence of Laves Phase in Nb-Hardened Superalloys

Superalloys 718, 625, 706 and Various Derivatives, ed. E. A. Loria, TMS, 1994, 683-694

38 N. Saunders and A.P. Miodownik

CALPHAD: A Comprehensive Guide

Elsevier Science Ltd. 1998.

39 N. Saunders and A.P. Miodownik

Phase Diagram Calculation for Ni-based Superalloys

Superalloys 1996, ed. R.D. Kissinger et al, TMS 1996, 101-110

40 Ling Yang

Phase Control in Nb-hardened Superalloys

M.S. Thesis, West Virginia University, 1997

41 T. Iida, et al

The Physical Properties of Liquid Metals

Claren Press, Oxford, U.K., 1988

42 A.F. Crawley

Densities of Liquid Metals and Alloys

Int. Met. Rev., 1974, vol. 19, 32-48

43 P.K. Sung, et al

Estimating Densities of Liquid Transition-Metals and Ni-base Superalloys

Materials Sci. Engr A, 1997, vol.A231, 189-197

44 M.R. Bridge and J. Beech

Formation of Macrosegregation in Large, As-cast masses: Direct Observation of Some Aspects

Solidification Technology in the Foundry and Cast House, The Metals Society, 1980, London, 478-483

45 K.P. Gupta et al

Phase Diagrams of Ternary Nickel Alloys

The Indian Institute of Metals, 1990

46 M.N. Gungor

A Statistically Significant Experimental Technique for Investigating Microsegregation in Cast Alloys

Metall Trans A., 1989, vol. 20A, 2529-2533

47 H. Fredriksson and B. Rogberg

Thermal Analysis for Interpretation of Solidification Cycle

Metal Science, Dec.1979, 685-690

48 H.D. Brody and M.C. Flemings

Solute Redistribution in Dendritic Solidification

Trans AIME, 1966, vol. 236, 615-624

49 J.A. Sarreal and G.J. Abbaschian

Metall Trans A., 1986, vol. 17A, 2063-2073

50 M. Hirai

Estimation of Viscosities of Liquid Alloys

ISIJ International, 1993, vol.33, 251-258

51 K.C. Mills and P.N. Quested

Measurements of the Physical Properties of Liquid Metals

Liquid Metal Processing and Casting Conf.Proc, ed. A.Mitchell, AVS, 1994, 226-240

52 D.R. Poirier

Permeability for Flow of Interdendritic Liquid in Columnar-Dendritic Alloys

Metall Trans B, 1987, vol.18B, 245-255

53 W.Yang, W.Chen and K.-M. Chang

Composition Effects on Macroscopic Solidification Segregation of Ni-based Superalloys

Annual Report II submitted to Special Metals Corporation, 1999.

54 G. A. Knorovsky, et al.

INCONEL 718: A Solidification Diagram

Metall. Trans. A, 1989, v.20A, 2149-2158 